\title{
Fluid instabilities in precessing ellipsoidal shells
}

\author{
Dissertation \\ zur Erlangung des Doktorgrades \\ der Mathematisch-Naturwissenschaftlichen Fakultäten \\ der Georg-August-Universität zu Göttingen
}

vorgelegt von

Silvia Lorenzani

aus

Terni (Italien)

Göttingen 2001 
D 7

Referent: Prof. Dr. A. Tilgner

Korreferent: Dr. M. Rein

Tag der mündlichen Prüfung: 13.11.2001 


\section{Contents}

$\begin{array}{lll}0 & \text { Motivation and summary } & 5\end{array}$

1 Fundamentals of rotating fluids theory $\quad 9$

1.1 Inertial waves: Rossby waves . . . . . . . . . . . . . . . 9 9

1.2 Ekman boundary layer . . . . . . . . . . . . . . . . . . . . . . . . . . . . . . . . . . . . . .

1.3 Internal shear layers . . . . . . . . . . . . . . . . 26

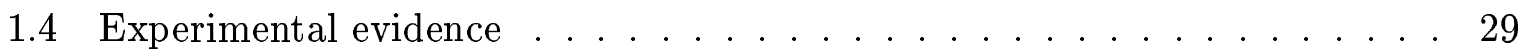

2 Viscous instabilities $\quad 35$

2.1 Equations of motion and analytical results . . . . . . . . . . . . . 35

2.1.1 Poincaré solution . . . . . . . . . . . . 37

2.1 .2 Busse solution . . . . . . . . . . . . . . 39

2.2 Numerical methods . . . . . . . . . . . . . . . . 41

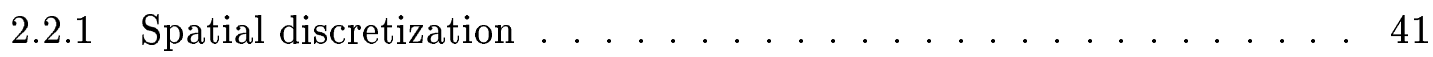

2.2.2 Temporal discretization . . . . . . . . . . . . . . 47

2.3 Laminar flows . . . . . . . . . . . . . . . . . . . . . . . . . . . . . . . . . . . . . . . . . . . . .

2.4 Instabilities . . . . . . . . . . . . . . . . . . . 59

2.4.1 Ekman layer instability .................. 71

3 Precession driven dynamo $\quad \mathbf{7 7}$

3.1 Introduction . . . . . . . . . . . . . . . . . 77

3.2 Mathematical formulation and numerical methods . . . . . . . . 79

3.3 Kinematic dynamo problem . . . . . . . . . . . . 82

4 Inertial instabilities $\quad \mathbf{8 7}$

4.1 Theoretical background . . . . . . . . . . . . . . 87

4.2 Experimental evidence . . . . . . . . . . . . . . . . . . . . . . . . . . . . . . . . . 92

4.3 Numerical methods . . . . . . . . . . . . . . . . . . . . . . . . . . . . . . . . . . . . .

4.4 Instabilities . . . . . . . . . . . . . . . . . . . . . . . . . . . . . . . . . . . . .

4.5 Hyperviscosity . . . . . . . . . . . . . . . . . . . . . . . . . . . . . . . . . .

5 Conclusions and perspectives $\quad 123$ 
$\begin{array}{lr}\text { A A practical implementation } & 127\end{array}$

A.1 Spatial discretization . . . . . . . . . . . . . . . 127

A.2 Temporal discretization . . . . . . . . . . . . . . 131

A.3 Parallel implementation . . . . . . . . . . . . . . 133

$\begin{array}{ll}\text { B Energies in ellipsoidal cavities } & 135\end{array}$ 


\section{Chapter 0}

\section{Motivation and summary}

The study of precession-driven flows in ellipsoidal containers has excited over the years a great although not constant interest. The first investigations related to this matter date back to Poincaré (1910), whose research was firstly directed to find out whether the Earth is entirely solid or contains a liquid interior. He concluded that the Earth must be entirely solid because a liquid core would shorten the period of nutations in comparison with the Eulerian periods of a solid top, whereas the observed periods are somewhat longer. Only the intense work of geophysicists over several years gave the knowledge that indeed other factors, besides the core, have influence on the Earth's rotation, as the elasticity of the mantle, the motion of the oceans and even the atmosphere. Liquid in the Earth's interior has been discovered only later by seismology.

To give an idea of the parameters involved in every realistic application to the Earth, a short summary of the most important features of our planet will be presented. According to our modern knowledge, the Earth consists of three layers: a solid silicate mantle followed by liquid material (mainly molten iron with some admixture of lighter elements, most likely sulphur or silicon). The most internal region contains again a solid consisting mainly of iron which is alloyed with nickel. The two interior layers are designated as outer and inner core. The physical properties of iron at core pressures of a few megabars are not very well known, and estimates for the viscosity in the outer core vary by several orders of magnitude. The electrical conductivity is somewhat better known even though a factor 3 must be regarded as a realistic estimate for the uncertainty. Some of the commonly used values for the properties of the Earth are listed in table 1.

After Poincaré, geophysics was the main motivation for further investigations on precession driven flows in connection with the origin of the Earth's magnetic field (Bullard, 1949). Nowadays it is generally accepted that the Earth's magnetic field is generated by motions in the liquid outer core, but the details of this process are still matter of investigation. The generation of magnetic fields by motions in an electrically conducting fluid is called dynamo action. This process converts mechanical energy into magnetic energy and dissipates it in the form of ohmic heat. Most geophysicists regard convection driven by thermal or chemical buoyancy as the most likely source of energy for the geodynamo, but the possibility of a dynamo driven by the Earth's precession cannot be rejected. The fea- 


\begin{tabular}{||l|r||}
\hline$r_{I C}$ & $1220 \mathrm{~km}$ \\
\hline$r_{C M B}$ & $3480 \mathrm{~km}$ \\
\hline$r_{E}$ & $6370 \mathrm{~km}$ \\
\hline$e_{I C}$ & $1 / 414$ \\
\hline$e_{C M B}$ & $1 / 393$ \\
\hline$\Omega_{p} / \omega_{D}$ & $-1.07 \times 10^{-7}$ \\
\hline$\alpha$ & $23.5^{\circ}$ \\
\hline$\nu$ & $10^{-6} \mathrm{~m}^{2} / \mathrm{s}$ \\
\hline$\sigma$ & $5 \times 10^{5} \mathrm{~S} / \mathrm{m}$ \\
\hline
\end{tabular}

Table 1: Some of the commonly used values for the properties of the Earth. The average radii of the inner core, $r_{I C}$, the core mantle boundary (CMB), $r_{C M B}$, and the entire Earth, $r_{E}$, along with the ellipticities of the inner core and the CMB, $e_{I C}$ and $e_{C M B}$, are taken from ref. [48]. The ellipticities are defined as $e=1-c / a$, where $a$ and $c$ are the major and minor axes, respectively. The Earth precesses in retrograde motion about the normal to the ecliptic with a period $2 \pi / \Omega_{p}$ of $25700 \mathrm{yr}$, while the diurnal rotation period $2 \pi / \omega_{D}$ equals 1 day. $\alpha$ is the angle between the rotation axis and the normal to the ecliptic. Estimates for the viscosity $\nu$ vary widely, see ref. [56]. The conductivity $\sigma$ is from ref. $[35]$.

sibility of a precession-driven geodynamo has been investigated by Malkus $(1963,1968)$. The advantage of this suggestion is in the well known character of the forces involved. Malkus based his proposal on the assumption that the flow in the core becomes turbulent.

Poincaré (1910) first found an analytical solution for the motion of an ideal fluid in a precessing spheroidal cavity, which he rederived after it had been derived by Hough (1895) fifteen years earlier. He showed that flows with constant vorticity are solutions of the equation of motion and suggested a negligible effect of precession on motions in the Earth's core. Later Busse (1968) proved that this solution does not represent the solution of the problem for a viscous fluid when viscosity tends to zero. In this limit, instead of a constant vorticity, a singularity in the form of a cylindrical vortex sheet develops. Malkus (1968) has demonstrated by laboratory experiments that the modifications of the Poincaré solution in terms of internal shear layers become unstable to wavy disturbances and that a fully turbulent state is realized when the rate of precession is sufficiently large.

It is doubtful whether the precession of the Earth is strong enough to induce a turbulent state in the core. On the other hand, a precessional origin of geomagnetism is an attractive hypothesis in terms of energy considerations, since the vast reserve of the rotational energy of the Earth would be available. Dolginov (1976) considered precession as the common origin of planetary magnetism.

Perturbations of uniformly rotating fluids, in particular due to precession, have been studied not only by the geophysical but also by the engineering community in connection with the control of satellites. Spacecrafts are frequently stabilized by spinning them about an axis. When the orientation of the spin axis needs to be changed during some maneuver, the reaction of the liquid fuel contained in them must be taken into account. 
These purposes led to experiments with precessing containers in many respects similar to those motivated by geophysical problems (Vanyo, Likins, 1971; Vanyo et al., 1995).

Beyond any practical application, rotating fluids can be considered a rich matter of investigation for the multiplicity of flow patterns which arise. The research work presented in this report intends to examine, by direct numerical simulation, the fluid motions existing in precessing ellipsoidal cavities and eventually investigate whether the generated velocity fields are suitable to support dynamo action.

Chapter 1 is devoted to a brief review of the basic properties of bounded rotating fluids in both the inviscid limit (inertial waves) and the viscous regime (Ekman boundary layers, internal shear layers). In the last section, the main experimental results obtained in precessing spheroidal cavities have been summarized.

In chapter 2, the equations of motion describing precession-driven flows in ellipsoidal shells are presented along with some analytical results (Poincaré, 1910; Busse, 1968) (section 2.1). The numerical methods employed in order to discretize the equations of motion are introduced in section 2.2, while in sections 2.3 and 2.4 the laminar flows obtained at low precession rates and the instabilities of the internal viscous shear layers at higher precession rates, as well as those originated in the Ekman boundary layers, are studied.

In chapter 3, a first attempt in simulating a precession-driven dynamo has been done, within the kinematic approach.

Chapter 4 describes a further instability mechanism related to the straining fields experienced by the inviscid Poincaré's solution of the Navier-Stokes equation. After a brief description of the theoretical background (section 4.1) and a short summary of the experimental evidence of inertial instabilities (section 4.2), an extension of the numerical methods presented in section 2.2 has been introduced in order to switch from no-slip to free-slip boundary conditions (section 4.3). The inertial instabilities detected numerically have been described in section 4.4, while section 4.5 reports upon an investigation of unstable flows made at low Ekman numbers by means of 'hyperviscosity'.

The general conclusions and the perspectives for future work have been drawn, finally, in chapter 5 . 


\section{Chapter 1}

\section{Fundamentals of rotating fluids theory}

\subsection{Inertial waves: Rossby waves}

The equations governing the motion of an incompressible viscous fluid rotating with constant angular velocity around the $z$-axis $\left(\boldsymbol{\omega}_{D}=\omega_{D} \hat{\boldsymbol{z}}\right)$ read:

$$
\begin{gathered}
\frac{\partial \boldsymbol{u}}{\partial t}+(\boldsymbol{u} \cdot \nabla) \boldsymbol{u}+2 \boldsymbol{\omega}_{D} \times \boldsymbol{u}=-\nabla p+\nu \nabla^{2} \boldsymbol{u} \\
\nabla \cdot \boldsymbol{u}=0
\end{gathered}
$$

where $\nu$ denotes the viscosity of the fluid and $p$ the pressure. Under the assumptions that the motion is:

- slow $[(\boldsymbol{u} \cdot \nabla) \boldsymbol{u} \simeq 0]$;

- $\operatorname{steady}[\partial \boldsymbol{u} / \partial t=0]$;

- inviscid $[\nu=0]$;

the momentum equation reduces to:

$$
2 \omega_{D} \hat{\boldsymbol{z}} \times \boldsymbol{u}=-\nabla p
$$

By taking the curl of (1.3) and using the incompressibility relation (1.2), it follows immediately:

$$
\omega_{D}(\hat{\boldsymbol{z}} \cdot \nabla) \boldsymbol{u}=0
$$

Equation (1.4) expresses a profound result known as Proudman-Taylor theorem (thereafter PT theorem): under the conditions above mentioned, the flow of a rotating fluid is two-dimensional:

$$
\boldsymbol{u}=\boldsymbol{u}(x, y)
$$


An experimental proof of this theorem is given by the flow of a fluid filling a layer $0 \leq z \leq d$ with uniform depth $d$ apart from a mound (or trough) where the depth is less (or greater) than $d$. The resulting stream is such that the fluid flows as though the mound completely fills a cylinder having generators parallel to the angular velocity of the container and a base defined by the periphery of the mound. A stagnant column of fluid stands above the mound enforcing the stream to flow around it (Taylor column). A motion over the small mound would require a change in column height. The inviscid boundary condition at the walls, $\boldsymbol{u} \cdot \hat{\boldsymbol{n}}=0$, implies that the vertical velocity component is zero at the top wall but different from zero on the protuberance. Since this component is independent of height, according the $\mathrm{PT}$ theorem, the contradiction is evident and no flow of this sort is possible. Hide (1966) proposed that undulations on the CMB (core-mantle boundary) of approximately a kilometre in height influence core flows to a considerable depth because of the PT theorem, giving a significant contribution to the coupling between core and mantle.

The generalization of the foregoing analysis to include unsteadiness of the flow leads to $([28])$ :

$$
\frac{\partial \boldsymbol{u}}{\partial t}=-2 \boldsymbol{\omega}_{D} \times \boldsymbol{u}-\nabla p
$$

If we consider a fluid bounded within a fixed surface $S$ (whose outwardly directed unit normal is $\hat{\boldsymbol{n}})$ :

$$
\hat{\boldsymbol{n}} \cdot \boldsymbol{u}=0 \quad \text { on } \mathrm{S}
$$

(inviscid boundary condition).

Taking the curl of (1.5) and denoting the vorticity $\nabla \times \boldsymbol{u}$ by $\boldsymbol{\omega}$, we obtain (taking into account the incompressibility of the flow):

$$
\frac{\partial \boldsymbol{\omega}}{\partial t}=-2 \nabla \times\left(\boldsymbol{\omega}_{D} \times \boldsymbol{u}\right)=2 \omega_{D}(\hat{\boldsymbol{z}} \cdot \nabla) \boldsymbol{u}=2 \omega_{D} \frac{\partial \boldsymbol{u}}{\partial z}
$$

A further curl of (1.7) gives:

$$
\frac{\partial}{\partial t} \nabla^{2} \boldsymbol{u}=-2 \omega_{D} \frac{\partial \boldsymbol{\omega}}{\partial z}
$$

A time-differentiation of (1.8) together with (1.7) leads to Poincaré's equation:

$$
\frac{\partial^{2}}{\partial t^{2}} \nabla^{2} \boldsymbol{u}=-4 \omega_{D}^{2} \frac{\partial^{2} \boldsymbol{u}}{\partial z^{2}}
$$

This means that equation (1.5) possesses wave-like solutions. Likewise, it is possible to show that the pressure and the vorticity also obey Poincare's equation.

For the pressure.

The divergence of (1.5) gives:

$$
\nabla^{2} p=-2 \nabla \cdot\left(\boldsymbol{\omega}_{D} \times \boldsymbol{u}\right)=2 \boldsymbol{\omega}_{D} \cdot \boldsymbol{\omega}
$$


A time-differentiation with the help of equations (1.7) and (1.5) leads us to the conclusion:

$$
\frac{\partial^{2}}{\partial t^{2}} \nabla^{2} p=4 \omega_{D}^{2} \hat{\boldsymbol{z}} \cdot(\hat{\boldsymbol{z}} \cdot \nabla) \frac{\partial \boldsymbol{u}}{\partial t}=4 \omega_{D}^{2} \hat{\boldsymbol{z}} \cdot(\hat{\boldsymbol{z}} \cdot \nabla)\left(-\nabla p-2 \boldsymbol{\omega}_{D} \times \boldsymbol{u}\right)=-4 \omega_{D}^{2} \frac{\partial^{2} p}{\partial z^{2}}
$$

For the vorticity.

Taking the curl and the curl of the curl of (1.7):

$$
\begin{aligned}
\frac{\partial}{\partial t} \nabla \times \boldsymbol{\omega} & =2 \omega_{D} \frac{\partial \boldsymbol{\omega}}{\partial z} \\
\frac{\partial}{\partial t} \nabla \times(\nabla \times \boldsymbol{\omega}) & =2 \omega_{D} \frac{\partial}{\partial z} \nabla \times \boldsymbol{\omega}
\end{aligned}
$$

it follows:

$$
-\frac{\partial}{\partial t} \nabla^{2} \boldsymbol{\omega}=2 \omega_{D} \frac{\partial}{\partial z} \nabla \times \boldsymbol{\omega}
$$

A time-differentiation with the help of (1.7):

$$
\frac{\partial^{2}}{\partial t^{2}} \nabla^{2} \boldsymbol{\omega}=-2 \omega_{D} \frac{\partial}{\partial z} \nabla \times \frac{\partial \boldsymbol{\omega}}{\partial t}=-4 \omega_{D}^{2} \frac{\partial^{2} \boldsymbol{\omega}}{\partial z^{2}}
$$

Inserting the periodic solutions

$$
\boldsymbol{u}=\Re_{e}\left(\boldsymbol{u}^{\prime} e^{-i \lambda t}\right) \quad p=\Re_{e}\left(p^{\prime} e^{-i \lambda t}\right)
$$

in (1.5) gives:

$$
-i \lambda \boldsymbol{u}^{\prime}=-\nabla p^{\prime}-2 \boldsymbol{\omega}_{D} \times \boldsymbol{u}^{\prime}
$$

By scalar-multiplying (1.12) by $\boldsymbol{u}^{\prime *}$ (the complex conjugate of $\boldsymbol{u}^{\prime}$ ), integrating over the volume of the container, and using the divergence theorem to eliminate $p^{\prime}$ with the help of the boundary and the incompressibility conditions, we can show that $\lambda$ is real and that:

$$
|\lambda| \leq 2 \omega_{D}
$$

(Greenspan, 1968, §2.7). For an unbounded fluid, considering plane-wave solutions of (1.5):

$$
\begin{aligned}
& \boldsymbol{u}=\Re_{e}\left(\boldsymbol{u}^{\prime} e^{i(\boldsymbol{k} \cdot \boldsymbol{r}-\lambda t)}\right) \\
& \boldsymbol{\omega}=\Re_{e}\left(\boldsymbol{\omega}^{\prime} e^{i(\boldsymbol{k} \cdot \boldsymbol{r}-\lambda t)}\right)
\end{aligned}
$$

we obtain from (1.7) (for simplicity all the primes will be omitted):

$$
\lambda \boldsymbol{\omega}=-2\left(\boldsymbol{\omega}_{D} \cdot \boldsymbol{k}\right) \boldsymbol{u}
$$

and from (1.8):

$$
\lambda \boldsymbol{u}=-\frac{2}{k^{2}}\left(\boldsymbol{\omega}_{D} \cdot \boldsymbol{k}\right) \boldsymbol{\omega}
$$


By elimination between (1.14) and (1.15):

$$
\lambda= \pm \frac{2}{k}\left(\boldsymbol{\omega}_{D} \cdot \boldsymbol{k}\right)= \pm 2\left(\boldsymbol{\omega}_{D} \cdot \hat{\boldsymbol{k}}\right)
$$

Inserting $\lambda$ in (1.14) it follows:

$$
\boldsymbol{\omega}=\mp k \boldsymbol{u}
$$

Therefore the phase velocity is:

$$
\boldsymbol{c}_{p} \equiv \frac{\lambda}{\boldsymbol{k}}= \pm 2 \frac{\boldsymbol{\omega}_{D} \cdot \hat{\boldsymbol{k}}}{k} \hat{\boldsymbol{k}}
$$

while the group velocity (the velocity of energy propagation) is given by:

$$
\boldsymbol{c}_{g}=\nabla_{k} \lambda(\boldsymbol{k})=\frac{\partial \lambda}{\partial k_{1}} \hat{\mathbf{i}}_{1}+\frac{\partial \lambda}{\partial k_{2}} \hat{\mathbf{i}}_{2}+\frac{\partial \lambda}{\partial k_{3}} \hat{\mathbf{i}}_{3}= \pm 2 \frac{\boldsymbol{\omega}_{D}}{k} \mp 2 \hat{\boldsymbol{k}} \frac{\left(\boldsymbol{\omega}_{D} \cdot \hat{\boldsymbol{k}}\right)}{k}= \pm 2 \frac{\boldsymbol{\omega}_{D}}{k} \mp \boldsymbol{c}_{p}
$$

where: $\boldsymbol{k}=k_{1} \hat{\mathbf{1}}_{1}+k_{2} \hat{\mathbf{1}}_{2}+k_{3} \hat{\mathbf{i}}_{3}$, or equivalently:

$$
\boldsymbol{c}_{g}= \pm 2 \frac{\hat{\boldsymbol{k}} \times\left(\boldsymbol{\omega}_{D} \times \hat{\boldsymbol{k}}\right)}{k}
$$

Thus the phase velocity is parallel to the wave fronts, while the group velocity is perpendicular to them. This result shows that the energy flux is at right angles to the phase velocity: a wave moving in one direction propagates energy in a perpendicular direction. These waves are evidently dispersive: the velocity at which their crests and troughs propagate is not a fixed quantity but depends upon wavelength. Note that the continuity equation implies:

$$
\boldsymbol{u} \cdot \boldsymbol{k}=0
$$

i.e., the velocity vector is perpendicular to the propagation direction and the wave is transverse (as must be in incompressible flows). From (1.16) follows that as $\boldsymbol{\omega}_{D} \cdot \hat{\boldsymbol{k}} \longrightarrow 0$ then $\lambda \longrightarrow 0$, i.e. the waves are steady as they become two-dimensional (PT theorem).

When inertial waves are reflected off rigid boundaries, there is a change of wave number and a new direction of energy transport (the incoming and reflected flux vectors make equal angles with the rotation axis $\boldsymbol{\omega}_{D}$ ) that does not satisfy the Snell's law typical of non-dipersive system (where the incoming and reflected flux vectors make equal angles with the normal to the reflecting plane). In a viscous fluid there is an $O\left(E^{1 / 2}\right)$ loss in amplitude upon reflexion (where $E$ is the Ekman number). Thus a contained inertial wave packet is dissipated after $E^{-1 / 2}$ reflexions. The energy can be totally absorbed only if the reflected energy flux is parallel to the plane surface. This critical condition appears, in terms of the frequency, when:

$$
\lambda= \pm 2 \hat{\boldsymbol{\omega}}_{D} \cdot \hat{\boldsymbol{n}}
$$

Let us consider now the case of inertial waves of high azimuthal wave number in a rotating sphere $([28])$. Poincaré's equation for the pressure $p$ in cartesian coordinates can be written as:

$$
\frac{\partial^{2} p}{\partial x^{2}}+\frac{\partial^{2} p}{\partial y^{2}}-\left(\frac{4 \omega_{D}^{2}}{\lambda^{2}}-1\right) \frac{\partial^{2} p}{\partial z^{2}}=0
$$


In cylindrical coordinates $(s, \varphi, z)$ (which assume particular relevance for the present purposes) the latter equation becomes:

$$
\frac{\partial^{2} p}{\partial s^{2}}+\frac{1}{s} \frac{\partial p}{\partial s}+\frac{1}{s^{2}} \frac{\partial^{2} p}{\partial \varphi^{2}}-\left(\frac{4 \omega_{D}^{2}}{\lambda^{2}}-1\right) \frac{\partial^{2} p}{\partial z^{2}}=0
$$

Solving (1.12), the velocity field reads:

$$
\begin{aligned}
u_{s} & =\frac{1}{4 \omega_{D}^{2}-\lambda^{2}}\left[i \lambda \frac{\partial p}{\partial s}-\frac{2 \omega_{D}}{s} \frac{\partial p}{\partial \varphi}\right] \\
u_{\varphi} & =\frac{1}{4 \omega_{D}^{2}-\lambda^{2}}\left[\frac{i \lambda}{s} \frac{\partial p}{\partial \varphi}+2 \omega_{D} \frac{\partial p}{\partial s}\right] \\
u_{z} & =\frac{1}{i \lambda} \frac{\partial p}{\partial z}
\end{aligned}
$$

Inserting these three components in (1.6) (where in the present case $\hat{\boldsymbol{n}}=\nabla\left(x^{2}+y^{2}+z^{2}\right)$ ) which in cylindrical coordinates has the form:

$$
s u_{s}+z u_{z}=0
$$

one finally obtains:

$$
s \frac{\partial p}{\partial s}+\frac{2 i \omega_{D}}{\lambda} \frac{\partial p}{\partial \varphi}-\left(\frac{4 \omega_{D}^{2}}{\lambda^{2}}-1\right) z \frac{\partial p}{\partial z}=0
$$

on the spherical surface S. (The problem for the pressure alone was studied by Poincaré and named after him by Cartan). Equations (1.22) and (1.25) admit solutions of the form:

$$
p=p(s, z) e^{i(m \varphi-\lambda t)} \quad(m>0)
$$

Let us assume that these solutions have $z$ length scales large compared with their $s$ and $\varphi$ length scales. This means that $p$ is small everywhere except in a narrow cylinder surrounding $s=s_{o}$ [see figure 1.1].

This assumption can be justified a posteriori showing, by means a non-local analysis, that the eigenfunctions $p$ indeed vanish (if $m>>1$ ) at a very small distance from the cylinder $s=s_{o}$. In terms of our present local analysis, we can expand $p$ in power series of $m^{-1}$, the leading term being $p=p_{o}$. Inserting therefore

$$
p=p_{o}(s, z) e^{i(m \varphi-\lambda t)}
$$

in (1.22) we obtain:

$$
\frac{1}{\Lambda^{2}} \frac{\partial^{2} p_{o}}{\partial z^{2}}+p_{o}=0
$$

where:

$$
\Lambda=\frac{\lambda m}{2 s_{o} \omega_{D}}
$$




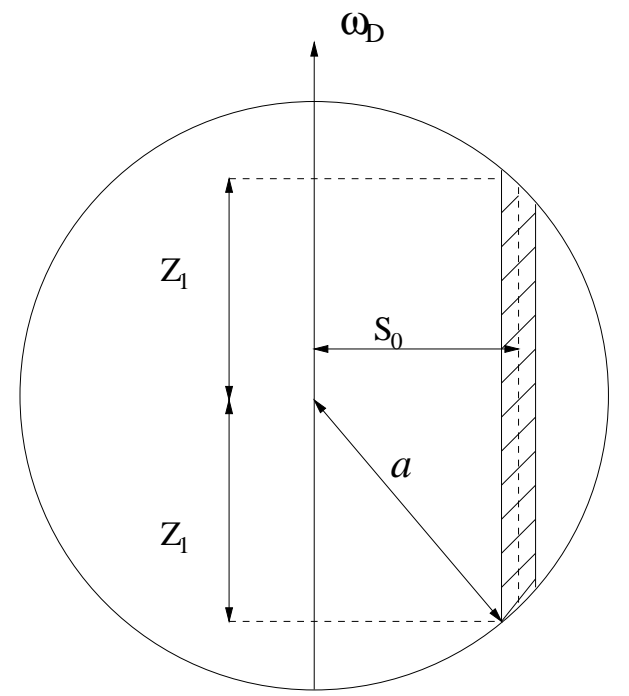

Figure 1.1: The localized nature of Rossby waves in a rotating sphere.

under the assumptions:

$$
\begin{gathered}
\frac{\partial^{2} p_{o}}{\partial s^{2}} \simeq \frac{\partial p_{o}}{\partial s} \simeq 0 \\
m>>1 \Rightarrow|\lambda|<<2 \omega_{D} .
\end{gathered}
$$

Likewise, the equation (1.25) on the boundary becomes:

$$
\frac{z}{s_{o} \Lambda} \frac{\partial p_{o}}{\partial z}+p_{o}=0
$$

which applies on the basis of the cylinder at $z= \pm z_{1}$ where $z_{1}=\left(a^{2}-s_{o}^{2}\right)^{1 / 2}$. The eigensolution of (1.27) is:

$$
p_{o}=f(s) \cos \left(\Lambda_{o} z\right)
$$

where $\Lambda_{o}$ is determined inserting (1.30) in (1.29):

$$
\Lambda_{o}=\frac{1}{z_{1}} \arcsin \left(\frac{s_{o}}{a}\right)
$$

with $0<\Lambda_{o}<\pi / 2$. From the definition (1.28), the frequency corresponding to the eigenvalue $\Lambda_{o}$ is:

$$
\lambda=\frac{2 \omega_{D} s_{o} \Lambda_{o}}{m}
$$

which is positive: the waves move eastwards, i.e. in the same sense as the rotation $\omega_{D}$ itself.

For the sake of completeness, having in mind a possible application to the Earth's outer core, we can extend the foregoing analysis to the case of a spherical annulus [see figure $1.2]$. 


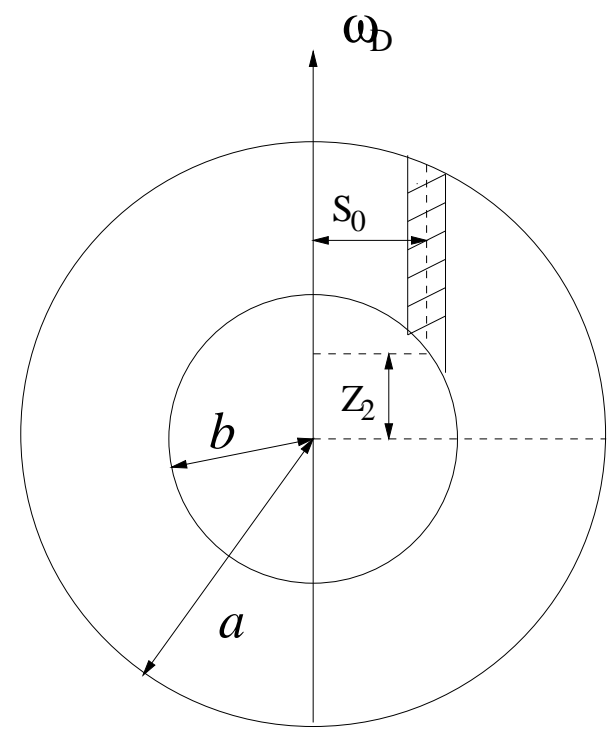

Figure 1.2: A localized Rossby wave in a spherical annulus.

The eigensolutions obtained performing a local analysis are still given by (1.30), but the boundary condition (1.29) must be applied at the two points: $z=z_{1}$ and $z=z_{2}=$ $\left(b^{2}-s_{o}^{2}\right)^{1 / 2}$. This generates an eigenvalue:

$$
\Lambda_{o}=-\frac{1}{z_{1}-z_{2}} \tan ^{-1}\left(\frac{s_{o}\left(z_{1}-z_{2}\right)}{s_{o}^{2}+z_{1} z_{2}}\right)
$$

which in the limit $|a-b|<<a$ of big inner core gives:

$$
\Lambda_{o}=-\frac{s_{o}}{a^{2}}
$$

leading to negative frequency:

$$
\lambda=-\frac{2 \omega_{D} s_{o}^{2}}{a^{2} m}
$$

The inertial waves in a spherical annulus move westward. The difference in sign between (1.32) and (1.35) can be explained by considering the vorticity of fluid elements as seen in a non-rotating inertial frame. The direction of wave propagation depends crucially on whether vortex filaments are lengthened or shortened as they move towards the rotation axis during an oscillation. If the former is the case, the waves move eastwards (fig.1.1); if the latter, they move westwards (fig.1.2).

\subsection{Ekman boundary layer}

Viscous processes force the fluid velocity to satisfy the no-slip boundary conditions at the solid walls of any arbitrarily shaped container. This adjustment occurs within a thin 
viscous layer, called Ekman layer after Ekman who first, at the beginning of the last century, discovered that the motion of a boundary is communicated to the fluid through a viscous boundary layer of thickness $E^{1 / 2}$. The rapid variation of flow components within the Ekman layer is often used to simplify the mathematical description of the system in that the tangential derivatives of any flow variable along the boundary can be neglected when compared with the normal derivative of the same function.

In order to illustrate the main characteristics of the Ekman layer steady flow, a simplified model will be examined ([74]). Consider the motion of a homogeneous, incompressible fluid rotating with angular velocity $\boldsymbol{\omega}_{D}$ about the $z$-axis. A rigid boundary perpendicular to $\boldsymbol{\omega}_{D}$ is placed at $z=0$.

A horizontally (along $\hat{\boldsymbol{x}}$ ) uniform geostrophic flow $U_{g}$ is specified far from the wall.

The boundary condition at $z \rightarrow \infty$, for the velocity $\boldsymbol{u}=(u, v, w)$, is:

$$
u=U_{g}, \quad v=w=0
$$

while at the rigid surface $z=0$, where the friction inhibits the fluid motion, the boundary condition is:

$$
u=v=w=0
$$

An exact solution of the equations of motion (1.1), (1.2) which satisfies (1.36) and (1.37) can be found in the form:

$$
\begin{aligned}
u & =u(z) \\
v & =v(z) \\
w & =w(z)
\end{aligned}
$$

because neither the boundary condition at $z=0$ nor the one at infinity requires lateral variations in the velocity fields. Inserting (1.38) into (1.2) yields:

$$
\frac{\partial w}{\partial z}=0
$$

which, with the condition that $w$ vanishes on $z=0$, implies that the vertical velocity is zero for all $z$, i.e.,

$$
w(z)=0
$$

Using (1.38) and (1.40), the three components of the momentum equation become:

$$
\begin{aligned}
-2 \omega_{D} v & =-\frac{1}{\varrho} \frac{\partial p}{\partial x}+\nu \frac{\partial^{2} u}{\partial z^{2}} \\
2 \omega_{D} u & =-\frac{1}{\varrho} \frac{\partial p}{\partial y}+\nu \frac{\partial^{2} v}{\partial z^{2}} \\
\frac{\partial p}{\partial z} & =0
\end{aligned}
$$


From the third equation of (1.41) follows:

$$
\frac{\partial}{\partial x} \frac{\partial p}{\partial z}=\frac{\partial}{\partial y} \frac{\partial p}{\partial z}=0
$$

or:

$$
\frac{\partial}{\partial z}\left\{\begin{array}{l}
\partial p / \partial x \\
\partial p / \partial y
\end{array}\right\}=0
$$

On the other hand, in the geostrophic region the governing equations are:

$$
\begin{aligned}
2 \omega_{D} U_{g} & =-\frac{1}{\varrho} \frac{\partial p}{\partial y} \\
0 & =-\frac{1}{\varrho} \frac{\partial p}{\partial x}
\end{aligned}
$$

Since the horizontal pressure gradient is independent of $z,(1.43)$ must also hold for all $z$. The horizontal pressure gradient is therefore determined entirely by the geostrophic velocity $U_{g}$ far from the boundary. Hence, inserting (1.43) into (1.41), the equations to be solved are:

$$
\begin{gathered}
-2 \omega_{D} v=\nu \frac{\partial^{2} u}{\partial z^{2}} \\
-2 \omega_{D}\left(U_{g}-u\right)=\nu \frac{\partial^{2} v}{\partial z^{2}}
\end{gathered}
$$

The solution is given by:

$$
\begin{aligned}
& u=U_{g}\left[1-e^{-z / \delta} \cos (z / \delta)\right] \\
& v=U_{g} e^{-z / \delta} \sin (z / \delta)
\end{aligned}
$$

where $\delta=\left(\nu / \omega_{D}\right)^{1 / 2}$ is the Ekman layer thickness. As $z \rightarrow \infty$ the velocities approach exponentially the interior, inviscid geostrophic flow. The region directly affected by friction is of the order of the Ekman layer thickness $\delta$. The stronger the rotation, the narrower the region directly affected by viscosity. Figure 1.3 shows the velocity vector $\boldsymbol{u}$ in the Ekman layer which is known as the Ekman spiral.

Different points along this spiral correspond to different values of $z / \delta$ and the velocity at each height is given in magnitude and direction by the line from the origin to the corresponding point on the spiral. As the boundary is approached:

$$
\lim _{z \rightarrow 0} \frac{v}{u}=1
$$

so that the velocity vector has turned $45^{\circ}$ to the left of the geostrophic velocity. A second noteworthy feature is that at $z / \delta=\pi$ the flow is in the same direction as the geostrophic flow but approximately 1.1 times as fast. 


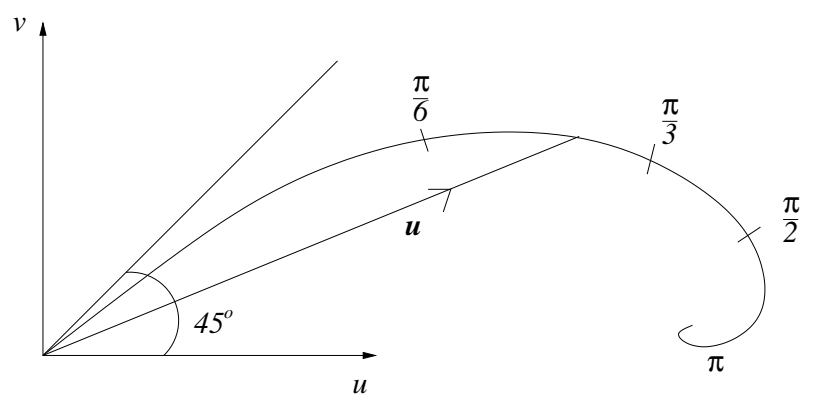

Figure 1.3: The Ekman spiral: polar diagram of the velocity vector $\boldsymbol{u}$ in the Ekman layer.

An Ekman layer flow has an additional important property, which leads to a significant interaction with the geostrophic region. This process can be intuitively explained as follows. Within the boundary layer, the Coriolis force is balanced by the viscous shear and the pressure is almost a constant. Thus, in steady flows, fluid particles close to the boundary are spun up to a larger angular velocity by direct viscous action. The increased Coriolis force overcomes the pressure gradient along the plate and the fluid is propelled radially outwards. To compensate for this mass flow, a small normal flux from the inviscid region is required. This process, known as Ekman layer suction, produces a secondary flow throughout the main body of the fluid. Transfer of fluid between boundary layers and the body of the fluid in this way is, for instance, the process by which a tank of fluid is brought to the angular velocity of its boundaries.

A classical procedure in describing viscous flows consists in adding to the inviscid solution a correction $\tilde{\boldsymbol{u}}$ which is significant only in the vicinity of the boundary. The boundary layer then produces a correction to the bulk flow which in turn modifies the boundary layer at higher orders in $E$, etc. leading to the following series expansion in powers of $E^{1 / 2}$ :

$$
\boldsymbol{u}=\boldsymbol{u}_{o}+\tilde{\boldsymbol{u}}_{o}+E^{1 / 2}\left(\boldsymbol{u}_{1}+\tilde{\boldsymbol{u}}_{1}\right)+\cdots
$$

The stability of this Ekman layer has been matter of experimental and theoretical investigations over the years.

Experiments were carried out by Faller(1963), Faller and Kaylor(1966) and Tatro and Mollö-Christensen(1967) (hereafter TMC). The former authors used a water-filled rotating tank with a free surface and the measurements were made by visual observations of the boundary layer circulation after the introduction of potassium permanganate dye crystals. The latter used an apparatus consisting of two parallel circular rotating plates forming a spool and utilized air as working medium. Their measurements, by means hot wire anemometers traversing the flow, were undoubtedly the most accurate.

To detect the onset of instability the hot wire probe was placed in the boundary layer and its signal monitored on an oscilloscope. Keeping all the experimental conditions fixed but incrementally increasing the mass flow through the tank, the hot wire voltage was recorded. At relatively small values of the flux, the recording line was essentially straight. 
As the mass flow was increased, however, oscillations appeared. The lowest value of the flux for which the oscillations were detected was taken as the critical point. For each combination of mass flow and rotation at which a critical point was determined, a Reynolds number and Rossby number based on the local measured values of the parameters were computed:

$$
R e=\frac{U_{g} \delta}{\nu}, \quad R o=\frac{U_{g}}{2 \omega_{D} r}
$$

where $\omega_{D}$ is the angular velocity of the tank, $r$ the radius, $\nu$ the viscosity, $U_{g}$ the relative azimuthal component of the interior velocity (geostrophic flow) and $\delta$ the local boundary layer thickness.

Since the theoretical Ekman radial velocity has a maximum at:

$$
\frac{z}{\delta}=\frac{\pi}{4}
$$

the boundary layer thickness was computed by measuring the vertical coordinate $z$ of the maximum radial velocity and taking:

$$
\delta=\frac{4 z}{\pi}
$$

The experiments showed conclusively that two kinds of instabilities exist.

The instability identified by Faller \& Kaylor as type II always appeared first and a linear regression which relates the critical Reynolds and Rossby numbers was found to be:

$$
R e_{I I_{c}}=56.3+116.8 R o
$$

At zero $R o$ the critical Reynolds number is: $56.3 \pm 2$.

The marked change in the shape of the oscillations with increasing the mass flow was interpreted by TMC as the appearing of a second kind of instability christened as type I by Faller(1963). A linear regression of the critical parameters for this instability was found to be:

$$
R e_{I_{c}}=124.5+7.32 R o
$$

which shows that in this case there is only a slight dependence of critical Reynolds number on the Rossby number. The waves of both families form a series of horizontal roll vortices. It was found experimentally that type II instabilities have a local orientation ranging from $0^{\circ}$ to $-8^{\circ}$ with respect to the geostrophic flow; their wavelengths vary between $25 \delta$ and $33 \delta$ and the phase speed is approximately $0.16 U_{g}$ directed radially inward. This instability first originated in the boundary layer but as the Reynolds number increased above the critical value it seemed to propagate throughout the entire interior region. TMC inferred that these are inertial waves excited by the boundary layer fluctuations.

Type I instabilities form an angle of $14.6^{\circ}$ with respect to the geostrophic flow, their wavelength is $11.8 \delta$ and the phase speed is $0.034 U_{g}$ directed radially inward. This instability appeared to be confined to the boundary layer. 
The type II instability occurring at lower Reynolds numbers was thought to have viscous origin, while the type I, always appearing at much higher $R e$, was referred to as the inviscid instability.

In order to perform a theoretical stability analysis of Ekman boundary layers ([25]), the appropriate dynamic equations for an incompressible, constant density, viscous flow in a rotating coordinate system, must be introduced after a proper choice of length and time scales:

$$
\begin{gathered}
R e\left[\frac{\partial \boldsymbol{u}}{\partial t}+\boldsymbol{u} \cdot \nabla \boldsymbol{u}\right]+2 \hat{\boldsymbol{z}} \times \boldsymbol{u}=-\nabla p+\nabla^{2} \boldsymbol{u} \\
\nabla \cdot \boldsymbol{u}=0
\end{gathered}
$$

It turns out that for the dimensionless equations, the Reynolds number $R e$ is the only parameter that appears explicitly.

Lilly(1966) investigated the stability of the two-dimensional boundary flow produced in a rotating tank by means of a perturbation analysis. The fluid at the lower boundary is assumed to be motionless and the rigid upper boundary located at infinity exerts no torque:

$$
\boldsymbol{u}=0 \quad \text { at } \quad z=0, \quad \hat{\boldsymbol{z}} \cdot \boldsymbol{u}=\hat{\boldsymbol{z}} \cdot \nabla \boldsymbol{u}=0 \quad \text { at } \quad z \longrightarrow \infty
$$

The stability analysis of a steady laminar motion $\boldsymbol{u}_{l}(\boldsymbol{r})$ which satisfies $(1.49),(1.50)$ and (1.51) can be performed by considering the growth of any small disturbance, $\xi \boldsymbol{u}^{\prime}(\boldsymbol{r}, t)$, superimposed on the mean flow. Substituting in (1.49), (1.50) the velocity and pressure functions written as:

$$
\begin{gathered}
\boldsymbol{u}=\boldsymbol{u}_{l}(\boldsymbol{r})+\xi \boldsymbol{u}^{\prime}(\boldsymbol{r}, t) \\
p=p_{l}(\boldsymbol{r})+\xi p^{\prime}(\boldsymbol{r}, t)
\end{gathered}
$$

and linearizing the equations with respect to the small amplitude parameter $\xi$, we obtain:

$$
\begin{gathered}
R e\left[\frac{\partial \boldsymbol{u}^{\prime}}{\partial t}+\boldsymbol{u}_{l} \cdot \nabla \boldsymbol{u}^{\prime}+\boldsymbol{u}^{\prime} \cdot \nabla \boldsymbol{u}_{l}\right]+2 \hat{\boldsymbol{z}} \times \boldsymbol{u}^{\prime}=-\nabla p^{\prime}+\nabla^{2} \boldsymbol{u}^{\prime} \\
\nabla \cdot \boldsymbol{u}^{\prime}=0
\end{gathered}
$$

The aim of this perturbation investigation is to determine whether the previous system has natural modes which amplify with time. If this is the case, then the basic flow is unstable because a small disturbance would receive energy from the mean motion sufficient to grow. The simplifying approximation introduced by Lilly, a fluid bounded by a single plane, is not restrictive in the sense that experimental results showed how the instabilities are dependent mainly on local flow conditions.

The structure of the boundary layer instability consists of two-dimensional vortex rolls therefore it is advantageous to consider a frame of reference in which the horizontal $x$ 


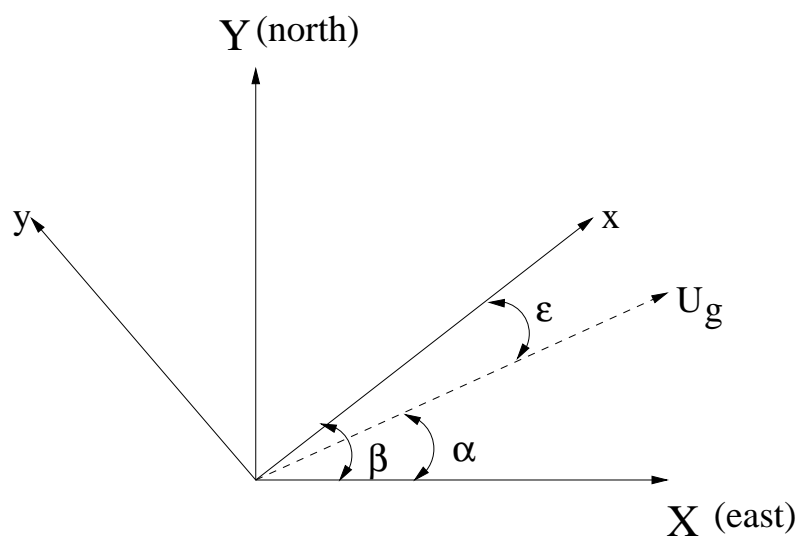

Figure 1.4: The coordinate system used in the presented study. $X$-axis points due east and $x$-axis points in the direction of the rolls (wave crests). $U_{g}$ is the direction of the geostrophic flow.

coordinates is along the band axis and $y$ is in the normal direction. The local $x$ axis is obtained from the direction of the geostrophic flow vector by a rotation through a positive (counter-clockwise) angle $\epsilon$ (see figure 1.4).

Lilly assumed also that derivatives of perturbation quantities vanish along the band axis $(\partial / \partial x=0)$. The dimensionless mean flow components in the laminar boundary layer are defined by:

$$
\boldsymbol{u}_{l}=U_{l} \hat{\mathbf{1}}+V_{l} \hat{\mathbf{j}}=\left[\cos \epsilon-e^{-z} \cos (\epsilon+z)\right] \hat{\mathbf{i}}-\left[\sin \epsilon-e^{-z} \sin (\epsilon+z)\right] \hat{\mathbf{j}}
$$

The perturbation boundary conditions remain essentially unchanged, i.e.,

$$
\begin{gathered}
u^{\prime}=v^{\prime}=w^{\prime}=0 \quad \text { at } \quad z=0 \\
\frac{\partial u^{\prime}}{\partial z}=\frac{\partial v^{\prime}}{\partial z}=w^{\prime}=0 \quad \text { at } \quad z=\infty
\end{gathered}
$$

The two-dimensional incompressibility condition (1.53) allows the introduction of a stream function defined by:

$$
v^{\prime}=\frac{\partial \psi^{\prime}}{\partial z}, \quad w^{\prime}=-\frac{\partial \psi^{\prime}}{\partial y}
$$

The pressure function is eliminated taking the curl of (1.52) to form a vorticity equation. Lilly assumed solutions for $\psi^{\prime}$ and $u^{\prime}$ periodic in $y$ and $t$ with phase and amplitude variable with $z$ :

$$
\begin{aligned}
& \psi^{\prime}(y, z, t)=\Psi(z) \exp [i k(y-c t)] \\
& u^{\prime}(y, z, t)=U(z) \exp [i k(y-c t)]
\end{aligned}
$$


where $k$ is the wave number and $c$ the complex phase speed:

$$
c=c_{r}+i c_{i}
$$

Inserting (1.55) and (1.56) in the $x$ components of the momentum and vorticity equations, leads to the following differential equations:

$$
\begin{gathered}
\frac{d^{2} U}{d z^{2}}-k^{2} U+2 \frac{d \Psi}{d z}-i k R e\left[\left(V_{l}-c\right) U-\Psi \frac{d U_{l}}{d z}\right]=0 \\
\left(\frac{d^{2}}{d z^{2}}-k^{2}\right)^{2} \Psi-i k \operatorname{Re}\left[\left(V_{l}-c\right)\left(\frac{d^{2}}{d z^{2}}-k^{2}\right) \Psi-\Psi \frac{d^{2} V_{l}}{d z^{2}}\right]-2 \frac{d U}{d z}=0
\end{gathered}
$$

with boundary conditions:

$$
\begin{gathered}
U=0 \quad \Psi=\frac{d \Psi}{d z}=0 \quad \text { at } \quad z=0 \\
\Psi=\frac{d^{2} \Psi}{d z^{2}}=\frac{d U}{d z}=0 \quad \text { at } \quad z=\infty
\end{gathered}
$$

Equations (1.57)-(1.60) define an eigenvalue problem in which the phase velocity $c$ is the eigenvalue, $\Psi$ and $U$ are the eigenfunctions and $\epsilon, k, R e$ specified parameters. Thus, for each eigenfunction mode, $c$ is a single-valued complex function of $k, R e$ and $\epsilon$. If the imaginary part of $c$ is positive for one or more of these modes, then the wave is unstable. The neutral stability surface:

$$
c_{i}(k, \epsilon, R e)=0
$$

establishes the boundary between stable and unstable conditions. Moreover, the line of greatest growth rate is defined as:

$$
\left.\frac{\partial\left(k c_{i}\right)}{\partial k}\right|_{R e}=\left.\frac{\partial\left(k c_{i}\right)}{\partial \epsilon}\right|_{R e}=0
$$

The critical surface and line of greatest growth rate intersect at the critical point, which defines the values of $k, \epsilon$ and $R e$ for the disturbance mode which becomes unstable at the lowest Reynolds number. In the limit of large Reynolds number (classical inviscid stability problem) equations (1.57) and (1.58) lead to:

$$
\begin{gathered}
U=\frac{\Psi}{V_{l}-c} \cdot \frac{d U_{l}}{d z} \\
\left(V_{l}-c\right)\left(\frac{d^{2}}{d z^{2}}-k^{2}\right) \Psi-\Psi \frac{d^{2} V_{l}}{d z^{2}}=0
\end{gathered}
$$

Rayleigh(1880) first proved that if the instability is of the inviscid type it must be associated with a point of inflection of the laminar velocity profile. Multiplying (1.62) 
and its complex conjugate by $\Psi^{+} /\left(V_{l}-c^{+}\right)$and $\Psi /\left(V_{l}-c\right)$ respectively, subtracting each other and integrating over the entire range of the vertical coordinate, one can obtain:

$$
\left(\Im_{m} c\right) \int_{z_{b}}^{z_{t}} \frac{\Psi \Psi^{+}}{\left(V_{l}-c\right)\left(V_{l}-c^{+}\right)} \frac{d^{2} V_{l}}{d z^{2}} d z=0
$$

If $\Im_{m} c>0$ (instability), then the integrand cannot be positive in the entire interval, therefore at some interior point:

$$
\frac{d^{2} V_{l}}{d z^{2}}=0
$$

It can be proved that this is only a necessary condition (therefore the same conclusion does not hold for stable flows, $\Im_{m} c<0$ ). Fjørtoft(1950) extended this condition proving that for instability it is also necessary for $\left|d V_{l} / d z\right|$ to be a maximum at the inflexion point. For the Ekman profile (1.54), the inflection points of the $V_{l}$ profile occur where:

$$
\cos (z+\epsilon) \equiv 0
$$

i.e.,

$$
z_{n}=\left(n+\frac{1}{2}\right) \pi-\epsilon \quad n=0,1,2, \cdots
$$

There are an infinity of such inflection points separated from each other by units of $\pi$, but for the condition on $\left|d V_{l} / d z\right|$ only the lowest inflection point is likely to have a measurable significance.

Neglecting the last term in (1.58), we obtain a truncated set which represents the classical approach (Orr-Sommerfeld equation). Lilly confirmed numerically that the type I instability is governed mainly by this classical equation. The type II instability, on the contrary, can arise only from a more complicated interaction that involves the Coriolis and shear forces. Lilly investigated the behaviour of this kind of instability (that he called "parallel instability") by means of a simplified analytic solution and found that these unstable waves vanish at high Reynolds numbers (when the Coriolis and shear forces tend to decouple) showing in this way their viscous character. The theoretical analysis reveals as well that the critical Reynolds number depends on $d U_{l} / d z$ : these disturbances arise mainly from the constant shear of the azimuthal velocity component.

The critical Reynolds numbers for the two different instability mechanisms found by Lilly solving numerically the complete set of equations $(1.57),(1.58)$ are:

$$
\begin{aligned}
& R e_{I I_{c}}=55 \text { for type II instability } \\
& R e_{I_{c}}=110 \text { for type I instability }
\end{aligned}
$$

Slightly supercritical Reynolds numbers (with respect to $R e_{I I_{c}}$ ) correspond to travelling waves with phase fronts oriented at angles of about $20^{\circ}$ to the right of the geostrophic flow (type II instability). At Reynolds number on the order of 100 a second mode of 
instability appears with very much smaller phase speeds and larger wavenumbers (type I instability). These latter disturbances have phase fronts (roll axes) aligned more closely with the geostrophic flow direction and to the left of it. The two types of unstable waves coexist for a range of Reynolds number in the sense that there are two local maxima of growth rate in the $(k, \epsilon)$-plane, with the type II ultimately disappearing at Reynolds numbers in the range $150-200$.

The experimental and theoretical investigations so far considered dealt only with horizontal Ekman layers. This is a suitable approximation when the vertical thickness of the fluid layer is much smaller than the horizontal length scales of the motion. This approach is indicated in literature as the "traditional problem" of Ekman layer stability. In the geophysical context however this approximation does not hold in that vertical and horizontal speeds are comparable.

Leibovich and Lele(1985) extended the theoretical work of Lilly to a spherical boundary ("full problem") and demonstrated the importance of the horizontal component of the angular velocity $\omega_{D}$ of rotation about the planetary axis. In the case of a horizontal Ekman layer the Coriolis accelerations which must be considered are those associated with $\omega_{D}^{N}$, the component of the angular velocity of the reference frame that is normal to the planes of motion.

In the geophysical context can be defined locally a plane of motion tangent to the Earth's surface at a given latitude $\lambda$ (neglecting the curvature of the Earth). In this case in addition to $\omega_{D}^{N}=\omega_{D} \sin \lambda$ there is a component of the angular velocity parallel to the tangent plane: $\omega_{D}^{H}=\omega_{D} \cos \lambda$. The full Ekman layer stability problem depends upon the same parameters already considered for the traditional approach, including in addition $\mu$, defined as follow:

$$
\mu=-\cot \lambda \cos \beta
$$

where $\beta$ is the counterclockwise angle made by the roll axis measured from east (see fig.1.4). The full and traditional problems require the solution of similar sixth-order differential systems in which Coriolis terms are $O\left(R e^{-1}\right)$ compared with inertial terms, therefore as $R e \longrightarrow \infty$ both are identical.

At finite Reynolds numbers, the qualitative behaviour found by Leibovich and Lele for the full problem is similar to Lilly's finding, except that the critical Reynolds number $\left(R e_{I I_{c}}\right)$ is smaller: it corresponds to 30.8 for latitude of $26.2^{\circ}$ and less. At $\lambda=90^{\circ}$ (traditional problem) $R e_{I I_{c}}=54.2$, which is in agreement with the value found by Lilly. [Note that the results obtained by Leibovich and Lele and reported here, refer to the atmospheric case because the oceanic investigation that they developed as well is believed not to be closely connected with the present context of this research]. The horizontal component of angular velocity acts therefore as a destabilizing agent. In addition, growth rates under slightly supercritical conditions are larger, the bands of unstable wave numbers much wider and for sufficiently large $\mu$, only one maximum of growth rate exists in the $(k, \epsilon)$-plane for moderate Reynolds numbers.

Recently, B.Desjardins, E.Dormy and E.Grenier (henceforth DDG) extended the previous works including the effects of a magnetic field (instability of Ekman-Hartmann boundary layers) in a parameter regime relevant to the Earth's liquid core. Having in 


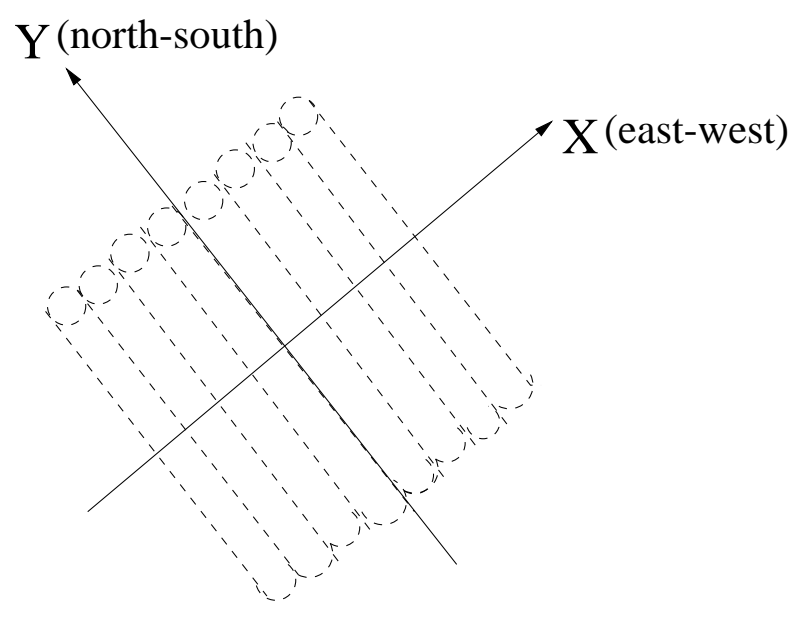

Figure 1.5: Sketch of the structure of the instability near the equator. In the polar region, the opposite direction of propagation is achieved.

mind a possible comparison (postponed to the next chapters) between all the previous investigations and the outcomes presented in this research, the main results obtained by DDG will be introduced in the limit of zero magnetic field (instability of a pure Ekman layer). Defining the width of the laminar Ekman boundary layer as a function of latitude:

$$
\delta=\sqrt{\frac{E}{\sin \lambda}}
$$

where $E$ is the Ekman number, a local boundary layer Reynolds number $R e$ can be estimated as:

$$
R e=\frac{2 \delta R o}{E}
$$

Note that at the pole $\left(\lambda=90^{\circ}\right), \delta=\sqrt{E}$ and $R e=\frac{2}{\sqrt{E}} R o$. The numerical simulations performed by DDG yielded upper bounds $R e_{i}$ such that if

$$
R e>R e_{i}
$$

the boundary layer flow is unstable. It was found that regardless of the magnetic field, there exists a critical band centered on the equator in which instabilities can occur. For geophysically relevant parameters this band extends below a critical latitude $\lambda_{c} \simeq 45^{\circ}$.

The physical characteristics of the instability (for $R e=R e_{i}$ ) reported by DDG have been summarized in figure 1.5: near the pole the instability is aligned with the east-west direction and propagates towards the pole; near the equator the instability is aligned with the north-south direction and propagates in the azimuthal direction. 


\subsection{Internal shear layers}

As observed previously (section 1.2), viscous processes force the fluid velocity to satisfy the no-slip condition on the boundary of the container and this adjustment occurs within a viscous boundary layer of thickness $E^{1 / 2}$.

Roberts \& Stewartson (1963) have demonstrated that the Ekman boundary layers, associated with any inertial wave, break down at a certain critical latitude (boundary layer 'eruptions', as Bondi \& Lyttleton christened them). This singularity appears wherever the oscillation frequency is related to the angular velocity of the container by:

$$
\lambda=2 \hat{\boldsymbol{n}} \cdot \hat{\boldsymbol{\omega}}_{D}
$$

where $\hat{\boldsymbol{n}}$ is the outward normal. This is exactly the same condition on frequency expressed in section 1.1, equation (1.20). Thus, the breakdown in the boundary layer at critical latitudes is connected with the total absorption of plane inertial waves in the boundary layer at these locations.

Roberts \& Stewartson (1963), analysing the steady flow in the neighbourhood of a critical position in a special problem, concluded that the boundary layer may be rescaled at the critical latitudes to give an increased thickness of $O\left(E^{2 / 5}\right)$ over a region of extent $O\left(E^{1 / 5}\right)$. The results of this linear analysis suggested that the total effect of these eruptions on the interior flow is negligible and non intrusive into the bulk of the fluid.

Moreover, using these scalings, the viscous correction to the frequency of the inertial waves due to the critical regions was found to be negligible compared to that from the rest of the Ekman layer (Greenspan 1968; Kerswell \& Barenghi 1995; Hollerbach \& Kerswell 1995).

Later Busse (1968), addressing the motion of a viscous fluid inside the spheroidal cavity of a precessing rigid body, included effects due to the non-linear terms in the boundary layer equation. He found that a shear layer is spawned in the mean flow at the critical radius, in good agreement with the experiments (Malkus 1968), suggesting that the critical regions are dynamically important for the interior flow. However, further experiments conducted by Vanyo et al.(1995) have evidenced the presence of vertical shear layers coaxial with the rotation axis of the fluid throughout the bulk of the flow.

Recently Kerswell (1995), using analytical results derived in a model problem based on the split-disk configuration studied previously by Stewartson(1957) and Walton(1975), has confirmed that the critical boundary regions spawn internal shear layers transporting angular momentum which penetrate into the bulk of the fluid. He has found that generally there are two characteristic directions along which a shear layer can be spawned at the boundary layer eruption: one directed back into the fluid and the other tangential to the boundary (with both inclined at $\tan ^{-1}\left[\lambda /\left(4-\lambda^{2}\right)^{1 / 2}\right]$ to the rotation axis). If the boundary is convex into the fluid at the critical latitude only one shear layer orientation is possible pointed back into the fluid. In this case the appropriate length scale would appear to be $O\left(E^{1 / 5}\right)$, i.e. the shear layer sees, as generating region, the whole lateral extent of the eruption. If the boundary is concave into the fluid at the critical latitude an additional shear layer is possible tangential to the boundary. This shear layer sees only the boundary 
layer thickness change of $O\left(E^{2 / 5}\right)$ (see figure 1.6). As demonstrated by Walton(1975) the "natural" width of such shear layers is $O\left(E^{1 / 3}\right)$. Thus, the non-tangential shear layers see a generating region thicker than their "natural" width $\left[O\left(E^{1 / 5}\right)>>O\left(E^{1 / 3}\right)\right]$, while the tangential shear layer sees a generating region thinner $\left[O\left(E^{2 / 5}\right)<<O\left(E^{1 / 3}\right)\right]$.

Kerswell(1995) proved that if the scale of variation is smaller than $E^{1 / 3}$, the shear layer formed does not see the fine structure in the boundary layer but rather only a discontinuity. Therefore the tangential shear layer will have a width $O\left(E^{1 / 3}\right)$ carrying velocities $O\left(E^{1 / 6}\right)$ [that is $O\left(E^{-1 / 3}\right)$ larger than in the general interior] whereas the nontangential shear layers will have a width $O\left(E^{1 / 5}\right)$ containing velocities $O\left(E^{3 / 10}\right)$ [that is $O\left(E^{-1 / 5}\right)$ larger than in the general interior].

Kerswell's analysis suggests that, instead of the usual partition of the flow domain into a boundary layer region [with velocities $O(R o)$ ] and an interior [with velocities $O\left(R_{o} E^{1 / 2}\right)$ ] (see equation (1.46)), a more appropriate general expansion is of the form:

$$
\boldsymbol{u}=R o\left[\boldsymbol{u}_{o}+\tilde{\boldsymbol{u}}_{o}+E^{1 / 6} \boldsymbol{u}_{\boldsymbol{s}}{ }^{t}+E^{3 / 10} \boldsymbol{u}_{\boldsymbol{s}}{ }^{n}+E^{1 / 2}\left(\boldsymbol{u}_{1}+\tilde{\boldsymbol{u}}_{1}\right)+O\left(E^{1 / 2}\right)\right]+O\left(R o^{2}\right)
$$

for the viscous velocity field due to an inertial wave, where velocities with a tilde are only significant in the boundary layer, $\boldsymbol{u}_{\boldsymbol{s}}{ }^{t}$ is non-zero only in the tangential shear layer and $\boldsymbol{u}_{\boldsymbol{s}}{ }^{n}$ is non-zero in the non-tangential shear layer. Thus, the new terms appearing in (1.66), $\boldsymbol{u}_{\boldsymbol{s}}{ }^{n}$ and $\boldsymbol{u}_{\boldsymbol{s}}{ }^{t}$, offer new possibilities for non-linear interactions in the interior flow.

In an accompanying paper, Hollerbach \& Kerswell(1995) presented a direct numerical solution (valid only in the weakly nonlinear regime) of the viscous spin-over mode (consisting of a rotation about an axis other than the rotation axis of the container) in spherical geometry. Since the spin-over inertial wave has the same frequency as the basic spin rate of the container, $\lambda=1$, the boundary layer eruptions occur at $\pm 30^{\circ}$ latitude and the internal shear layers spawned thereby are inclined at exactly $30^{\circ}$ to the container's axis of rotation. Their numerical simulations pointed out that once a tangential shear layer is spawned in the inner-core, the non-tangential shear layer does not appear: presumably the former sufficiently smooths over the eruption to make the latter redundant. The flow within these inclined shear layers decreases with decreasing Ekman number. Taking into account nonlinear responses, they showed how the flow exhibits axially aligned steady shear layers, which appear as cylinders parallel to the axis of rotation. The amplitude of these shear layers increases with decreasing Ekman number.

In addition to the mean flow shear layer at $s=\cos 30^{\circ}=0.86$ previously obtained by Busse, their numerical simulations revealed the presence of other vertical shear layers for $s<0.86$. As an explanation, Kerswell suggested that the boundary layer eruptions have not a local influence at the critical radius, but they affect the entire flow through obliquely inclined oscillatory shear layers which therefore are responsible for the appearence of a multitude of axial shear layers too.

Direct numerical simulations of precession-driven flow in a spherical shell by Tilgner (1999) revealed in addition to conical non-axisymmetric shear layers the existence of conical axisymmetric shear layers. Studying numerically the fluid motion in a slowly 

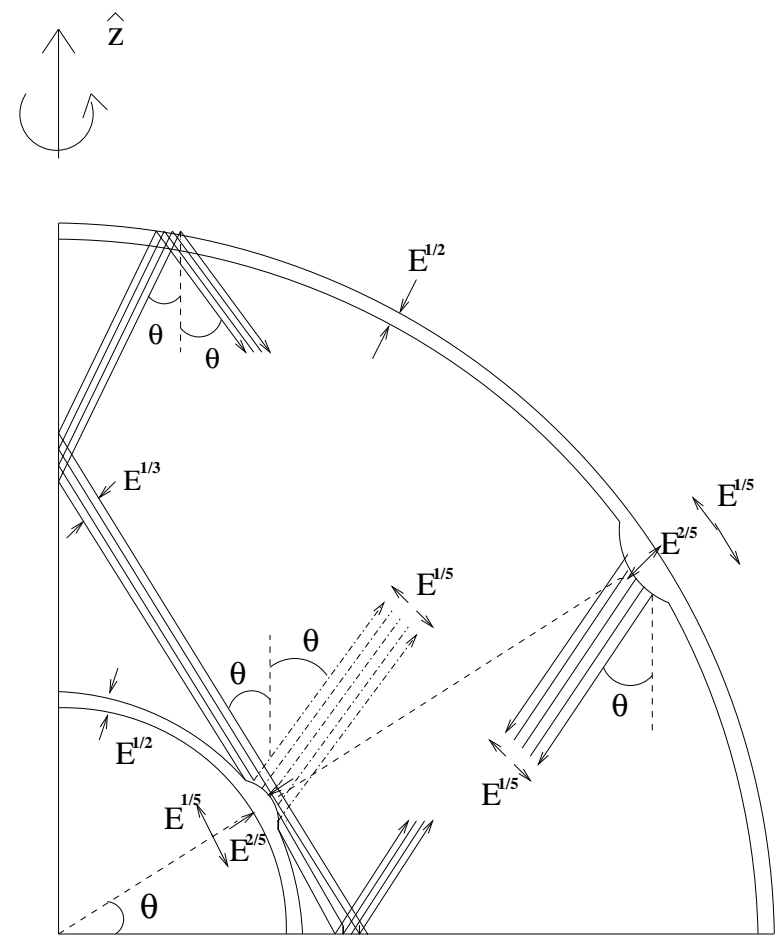

Figure 1.6: Sketch of the Ekman layer breakdown and of the internal layers spawned.

precessing ellipsoidal shell (Tilgner 1999) he pointed out that shear layers also arise in response to a difference in ellipticities between the inner and the outer boundary. The velocity gradients in these shear layers increase with decreasing Ekman number, whereas inclined shear zones due to an Ekman layer breakdown weaken with decreasing $E$.

But quoting Greenspan, "...even weak shear layers could provide a source of flow instability..."

Hide \& Titman (1967) studied experimentally the stability of detached shear layers surrounding an internal disk rotating at a different rate $\left(\boldsymbol{\omega}_{D}^{1}\right)$ from that of the external tank $\left(\boldsymbol{\omega}_{D}^{o}\right)$, completely filled with a homogeneous liquid of kinematical viscosity $\nu$. The experiments showed that, although the apparatus was axisymmetric, only when the Rossby number $R o$, defined as: $R o=2\left(\omega_{D}^{1}-\omega_{D}^{o}\right) /\left(\omega_{D}^{1}+\omega_{D}^{o}\right)$, did not exceed a critical value $R_{0}$, the flow displayed the same property of symmetry about the rotation axis. The empirical relationship found for the critical threshold was:

$$
\left|R o_{T}\right|=A E^{n}
$$

where $E$ is the Ekman number defined as: $E=2 \nu / a^{2}\left(\omega_{D}^{1}+\omega_{D}^{o}\right)$ ( $a$ is the radius of the internal disk) with $A$ and $n$ constant: $A=16.8 \pm 2.2, n=0.568 \pm 0.013$. When $|R o|>\left|R o_{T}\right|$, the layers become unstable. For $R o>0$ (i.e. $\omega_{D}^{1}>\omega_{D}^{o}$ ) a series of wavelike protuberances ( $M$ in number) showing little or no dependence on $z$ (two-dimensional 
vortex rolls) develop on the shear layer. $M$ generally decreases as $R o$ increases, but does not depend strongly on $E$. Apart from drifting uniformly about the axis of rotation of the apparatus at a rate intermediate between $\omega_{D}^{1}$ and $\omega_{D}^{o}$, the wave motion is steady.

When $R o<0$ the flow again becomes non-axisymmetric but the form of the disturbance is quite different. Under most conditions, instead of a wave-like pattern the unstable shear layer distorts into an off-axis ellipse. The critical value $R o_{T}$ does not depend on the sign of $R o$.

\subsection{Experimental evidence}

The first laboratory experiments intended to shed light on flow-patterns generated in precessing spheroidal cavity were performed by Malkus (1968). In a first experiment, a plastic water-filled spheroid, of major axis 25 centimeters and minor axis 24 centimeters, was rotated around its minor axis at 60 revolutions per minute. The spheroid and its spinning support were mounted on a larger horizontal precession table, with the minor axis of the spheroid inclined at 30 degrees to the vertical. In order to visualize the process, aluminum powder dispersed in the water was illuminated by a beam of light normal to the rotation axis.

Rotation of the precession table at $3 / 4$ revolution per minute gave rise to a steady two-dimensional flow, which showed a deviation from the general Poincaré solution in that zones of considerable shear appeared in the toroidal flow. In figure 1.7 is reported a sequence of photographic records, taken at 1-minute intervals in the equatorial plane, of a dye streak introduced into a laminar flow for an Ekman number of $2.5 \times 10^{-6}$ (based on the "diurnal" rotation rate $\omega_{D}$ of the ellipsoid about its symmetry axis).

The most evident feature of the toroidal flow is the retrograde motion in the interior (west-ward drift) and the retrograde and prograde "jets" on either side of radius $0.86\left(=\cos 30^{\circ}\right)$, which become more intense as the Ekman number is decreased. Data taken at Ekman number of $3 \times 10^{-7}$ exhibited prograde jets five times as intense as shown in figure 1.7. These toroidal flows developed a wavelike instability when the rotation of the precession table was increased at 1 revolution per minute, while rotation at $4 / 3$ revolution per minute caused a rather abrupt transition to turbulent flows. The instabilities of these shear flows were observed at values of:

$$
\varepsilon^{2} \geq 5 E^{1 / 2} F(E)
$$

where $\varepsilon$ is defined as the departure of the total velocity from solid-body rotation divided by the velocity of solid-body rotation (amplitude of the relative motion). In Malkus experiments $F(E) \sim 0.4$. A few laboratory observations (but no theoretical proofs) suggested that $F(E)$ could vary as $E^{-2 / 5}$ for very small Ekman numbers. Malkus concluded that if this scaling is applied to the Earth, flow in the Earth's core should be very unstable.

In a second experiment, a spheroid of major axis 7.5 centimeters and minor axis 6.75 centimeters was rotated at 900 revolutions per minute with the angle between the rotation and the precession axes $\alpha$ fixed at 96 degrees. The onset of a wave-like instability was 


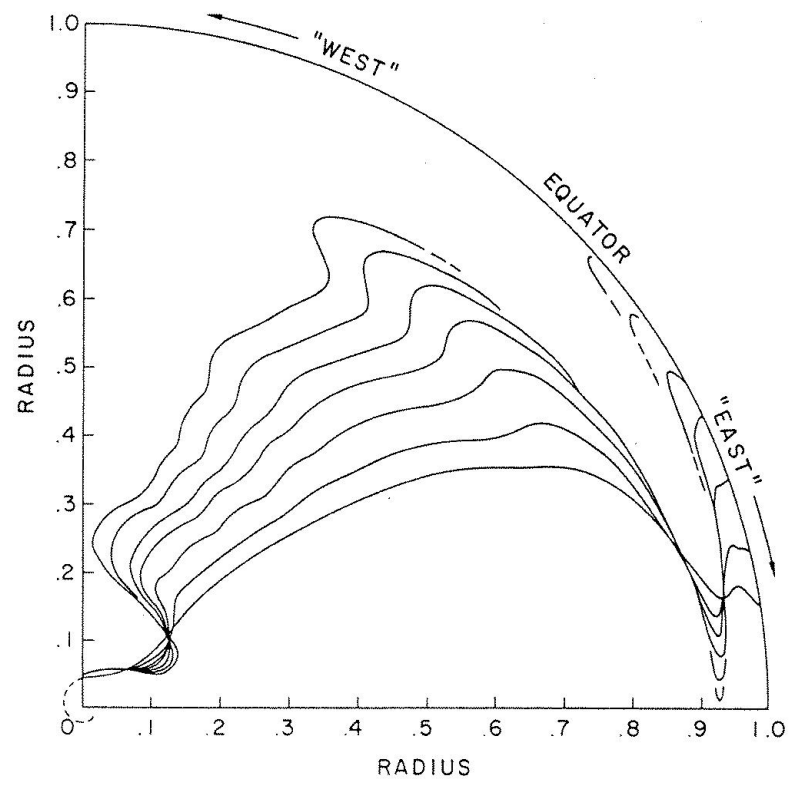

Figure 1.7: Tracing from photographic records showing the temporal evolution of a dye streak in the equatorial plane. The view is from the south pole, 'west' and 'east' are equivalent to 'retrograde' and 'prograde', respectively.

again observed, this time followed by a sudden transition to more violent motion during which the torque exerted by the motors of the experimental setup in order to maintain the precessional motion of the container abruptly increased. At this stage the fluid rotates about an axis almost parallel to the precession axis and the turbulence fluctuations are comparable in magnitude to the rotation velocity of the container. This second transition showed hysteresis.

In table 1.1 are summarized the parameters characterizing the two Malkus experiments mentioned above $\left(\Omega=\Omega_{p} / \omega_{D}\right.$ is the adimensional precession rate).

After Malkus, further experiments, matching more closely conditions expected in the Earth's outer core, were carried out by Vanyo et al. (1995). The experimental apparatus was similar to that of Malkus except they used a transparent, water-filled oblate spheroidal cavity, spinning at a rate of $\omega_{D}=200 \mathrm{rpm}$, with a major radius $R=0.252 \mathrm{~m}$ and an ellipticity $e=0.01$. The angle between the rotation and the precession axes was fixed at $\alpha=23.5^{\circ}$.

The experimental setup limited the precession rate $\Omega_{p}$ in the range $-11.5 \leq \Omega_{p} \leq$ $11.5 \mathrm{rpm}$, while structural strength of the thin wall cavity constrained $\omega_{D}$ to $200 \mathrm{rpm}$. Therefore, the smallest ratio $\Omega_{p} / \omega_{D}$ obtained in laboratory (the nearest to the Earth's ratio) was $0.1 / 200=5 \times 10^{-4}$. In a few experiments values of $\Omega_{p}=0.01 \mathrm{rpm}\left(\Omega_{p} / \omega_{D} \simeq\right.$ $5 \times 10^{-5}$ ) were reached at the cost of a loss of accuracy, but the results were similar to those at $\Omega_{p}=0.1 \mathrm{rpm}$. In Vanyo's experiments $E \simeq 8 \times 10^{-7}$.

Internal fluid motions were visualized by illuminating cross-sections of the spheroid 


$$
\begin{array}{rlrl} 
& \alpha=30^{\circ} & e=0.04 & E=2.5 \times 10^{-6} \\
|\Omega| \simeq 1.25 \times 10^{-2}: & & \text { steady } & \text { 2-dimensional flow } \\
|\Omega| \simeq 1.66 \times 10^{-2}: & \text { wave-like instability } \\
|\Omega| \simeq 2.22 \times 10^{-2}: & \text { turbulent flow } \\
& & \\
\alpha=96^{\circ} & e=0.1 & E=2 \times 10^{-6} \\
|\Omega|<1.33 \times 10^{-2}: & & \text { laminar region } \\
|\Omega| & \simeq 1.33 \times 10^{-2}: & & \text { wave-like instability } \\
2 \times 10^{-2}<|\Omega|<4.66 \times 10^{-2}: & \text { two-dimensional turbulence } \\
|\Omega| & \simeq 5.33 \times 10^{-2}: & & \text { saturated turbulence }
\end{array}
$$

Table 1.1: Malkus' experiments (1968).

$$
\begin{aligned}
\alpha & =23.5^{\circ} \quad & e=0.01 \quad E=8 \times 10^{-7} \\
|\Omega| & \simeq 5 \times 10^{-4}: & \text { laminar flow } \\
|\Omega| & \simeq 2.5 \times 10^{-3}: & \text { wave-like instability } \\
|\Omega| & \simeq 5.7 \times 10^{-2}: & \text { tubulent flow }
\end{aligned}
$$

Table 1.2: Vanyo et al. experiments (1995).

with sheets of parallel light approximately $2 \mathrm{~cm}$ thick. Microscopic flakes dispersed in the water-filled cavity showed random orientations except at shear surfaces. At low precession rates $\left(\Omega_{p} / \omega_{D}=-5 \times 10^{-4}\right)$ the motion is laminar and consists of two parts. The primary flow is an uniform rotation about an axis lagging the precessing spin axis of the container. This result of a fluid spin axis lagging the cavity axis was confirmed also using prograde precession.

The secondary flow is a differential rotation of nested cylinders axisymmetric with the liquid spin axis. The cylinders rotate alternately at faster and slower rates relative to the net retrograde motion of the fluid as a whole. In order to study more closely the velocities pattern, neutrally buoyant fluorescent dye was injected into the spheroid boundary layer at different latitudes after the onset of precession at $\Omega_{p}=-0.1 \mathrm{rpm}$. Two flows are superimposed to the retrograde motion, consistent with the interpretation of differentially rotating nested fluid cylinders. One motion is a meridional circulation which transports the dye both toward the pole and the equator. The second is a variation in westward drift rate with latitude.

Vanyo observed also two annuli of boundary layer spirals apparently zones of strong boundary layer divergence. These spirals were considered to represent Kelvin-Helmholtz or other shear flow induced instabilities between the differentially rotating cylinders.

As the precession rate increases, the fluid spin axis deviates from the cavity axis and 
slow non-axisymmetric columnar wave patterns develop between the rotating cylinders. Axial flows between the spheroidal cavity boundary and the interior are observed. A transition to turbulent flows was observed only at precession rates high enough to cause the fluid spin axis to align nearly with the precession axis. At $\Omega_{p} / \omega_{D}=-5 \times 10^{-2}$, for example, the motion is turbulent but elongated along the precession axis.

Vanyo's results related to the liquid spin axis orientations were in agreement with Busse's theoretical calculations in suggesting that the fluid spin axis should follow a nearly circular path, with increasing $\Omega_{p}$, having end points at the cavity spin axis and the precession axis.

Figure 1.8 summarizes the most significant phenomena observed in Vanyo's experiments.

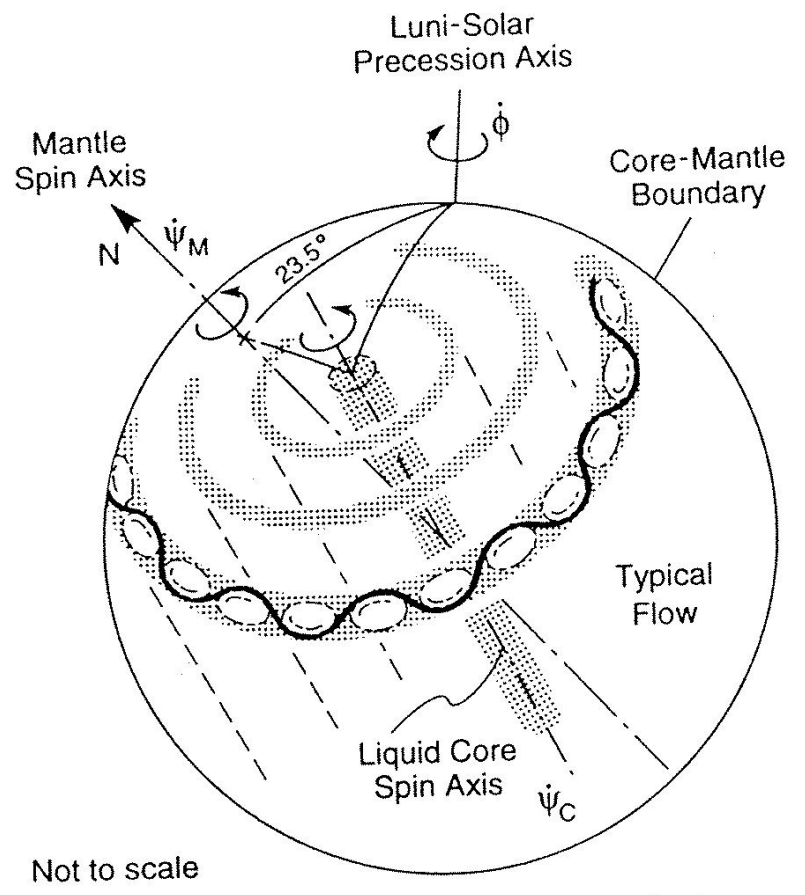

Figure 1.8: Sketch of the visual observations in Vanyo's experiments. The shaded areas indicate axisymmetric cylindrical shear layers. The wiggly line illustrates the deformations of these layers once the instability sets in.

A second set of experiments performed by Vanyo and Dunn (2000), using a liquid filled oblate spheroid spinning at $\omega_{D}=200 \mathrm{rpm}$ about an axis displaced $\alpha=23^{\circ} 27^{\prime}$ from the precession axis with ellipticity $e=1 / 400$ confirmed qualitatively the results obtained in the earlier research. No hysteresis phenomena were registered. The introduction of a solid core (not rotating relative to the spheroid) into the cavity of ellipticity $1 / 100$ used in earlier tests, showed the existence of a cylindrical shear surface tangent to the internal core. The tangent cylinder tended to separate flows inside and outside this cylinder. 
Vanyo's laboratory investigations, although in qualitative agreement with the early Malkus experiments, appeared to negate turbulence at parameters more closely related to the Earth's outer core conditions. In spite of that, the general conclusion concerning the feasibility of a precession-driven dynamo was substantially the same. In fact Vanyo, observing that precession induced large-scale axisymmetric zonal shears and smaller-scale columnar eddies are the same basic features observed in buoyancy-driven convection in rotating spheres, inferred that precessional flows can, in principle, produce a dynamo similar to a convection-driven dynamo. In table 1.2 are summarized the parameters characterizing some of the Vanyo experiments.

More recently new experiments have been carried out by Noir et al. (2001) in which a Doppler ultrasonic technique has been used in order to measure the fluid velocity. This method showed the non-axisymmetric internal shear layers predicted by theoretical investigations and numerical simulations, while the above mentioned experiments using flakes (Malkus, 1968) and dyes (Vanyo et al., 1995; Vanyo and Dunn, 2000) did not reveal them. 


\section{Chapter 2}

\section{Viscous instabilities}

\subsection{Equations of motion and analytical results}

This treatise deals with the investigation, by direct numerical simulations, of the motion of an incompressible fluid contained in an ellipsoid which rotates about its axis of symmetry and whose rotation axis is executing precessional motion. A natural approach to the problem consists, therefore, in deriving the Navier-Stokes equation in a frame of reference rotating with the instantaneous angular frequency $\omega_{\text {ref }}$ relative to an inertial frame.

The time derivatives of vectors in the two frames are related by:

$$
\left(\frac{\partial}{\partial t}\right)_{i n e r t}=\left(\frac{\partial}{\partial t}\right)_{r o t}+\omega_{r e f} \times
$$

Denoting with $\boldsymbol{r}$ the position vector of a fluid particle, it follows:

$$
\left(\frac{\partial \boldsymbol{r}}{\partial t}\right)_{\text {inert }}=\left(\frac{\partial \boldsymbol{r}}{\partial t}\right)_{r o t}+\boldsymbol{\omega}_{r e f} \times \boldsymbol{r}
$$

and the acceleration is written as:

$$
\begin{aligned}
\left(\frac{\partial^{2} \boldsymbol{r}}{\partial t^{2}}\right)_{\text {inert }} & =\left(\frac{\partial^{2} \boldsymbol{r}}{\partial t^{2}}\right)_{r o t}+2 \boldsymbol{\omega}_{r e f} \times\left(\frac{\partial \boldsymbol{r}}{\partial t}\right)_{r o t}+ \\
\boldsymbol{\omega}_{r e f} & \times\left(\boldsymbol{\omega}_{r e f} \times \boldsymbol{r}\right)+\left(\frac{\partial}{\partial t} \boldsymbol{\omega}_{r e f}\right)_{\text {inert }} \times \boldsymbol{r}
\end{aligned}
$$

The second and third terms on the right hand side correspond to the Coriolis and centrifugal forces well known in uniformly rotating systems, while the fourth term, peculiar to non-uniformly rotating frames, is generally referred to as the Poincaré force. In the inertial frame the Navier-Stokes equation reads:

$$
\left(\frac{\partial^{2} \boldsymbol{r}}{\partial t^{2}}\right)_{\text {inert }}=-\frac{1}{\rho} \nabla p+\nu \nabla^{2}\left(\frac{\partial \boldsymbol{r}}{\partial t}\right)_{\text {inert }}+\boldsymbol{f}_{\text {inert }}
$$


Inserting (2.2):

$$
\left(\frac{\partial^{2} \boldsymbol{r}}{\partial t^{2}}\right)_{\text {inert }}=-\frac{1}{\rho} \nabla p+\nu \nabla^{2} \boldsymbol{u}_{\text {rot }}+\boldsymbol{f}_{\text {inert }}
$$

where: $\boldsymbol{u}_{\text {rot }}=(\partial \boldsymbol{r} / \partial t)_{\text {rot }}, \nu$ is the viscosity of the fluid, $\rho$ its density and $p$ the pressure. $\boldsymbol{f}_{\text {inert }}$ is a volume force measured in the inertial frame.

Two different frames are particularly useful to address the problem of precession: one, attached to the container, is usually referred to as "mantle frame", the other, chosen such that the precession and rotation axes are stationary, will be called the "precession frame". The mantle rotates with a diurnal angular velocity $\boldsymbol{\omega}_{D}$ whose precession is described by the precession vector $\boldsymbol{\Omega}_{p}$. Thus, $\boldsymbol{\omega}_{\text {ref }}=\boldsymbol{\omega}_{D}+\boldsymbol{\Omega}_{p}$ and $\left(\partial \boldsymbol{\omega}_{r e f} / \partial t\right)_{\text {inert }}=\boldsymbol{\Omega}_{p} \times \boldsymbol{\omega}_{D}$ (Poisson's formula). Assembling the relations (2.3)-(2.4) and using the explicit expressions for $\boldsymbol{\omega}_{\text {ref }}$ and $\left(\partial \boldsymbol{\omega}_{\text {ref }} / \partial t\right)_{\text {inert }}$ given above together with the definition of "material derivative", the equation of motion in the mantle frame becomes (dropping subscripts):

$$
\frac{\partial \boldsymbol{u}}{\partial t}+(\boldsymbol{u} \cdot \nabla) \boldsymbol{u}+2\left(\boldsymbol{\omega}_{D}+\boldsymbol{\Omega}_{p}\right) \times \boldsymbol{u}=\nu \nabla^{2} \boldsymbol{u}-\nabla p_{\text {red }}-\left(\boldsymbol{\Omega}_{p} \times \boldsymbol{\omega}_{D}\right) \times \boldsymbol{r}+\boldsymbol{f}
$$

where:

$$
\nabla p_{\text {red }}=\frac{1}{\rho} \nabla p+\left(\boldsymbol{\omega}_{D}+\boldsymbol{\Omega}_{p}\right) \times\left[\left(\boldsymbol{\omega}_{D}+\boldsymbol{\Omega}_{p}\right) \times \boldsymbol{r}\right]
$$

The no-slip boundary condition at the outer boundary is: $\boldsymbol{u}=0$.

In the precession frame (where $\boldsymbol{\omega}_{\text {ref }}=\boldsymbol{\Omega}_{p}$ ), $\boldsymbol{\omega}_{D}$ and $\boldsymbol{\Omega}_{p}$ are stationary and consequently the Poincaré force disappears, so that the equation of motion reads:

$$
\frac{\partial \boldsymbol{u}}{\partial t}+(\boldsymbol{u} \cdot \nabla) \boldsymbol{u}+2 \boldsymbol{\Omega}_{p} \times \boldsymbol{u}=\nu \nabla^{2} \boldsymbol{u}-\nabla p_{\text {red }}+\boldsymbol{f}
$$

The no-slip boundary condition at the outer boundary is: $\boldsymbol{u}=\boldsymbol{\omega}_{D} \times \boldsymbol{r}$.

If $\boldsymbol{f}$ is a gravitational force (therefore deriving from a potential), then it can be absorbed in the pressure gradient. When the motion of the container is prescribed (in the case of the Earth, a precessional motion), gravitational forces have no influence on the fluid flow.

Choosing as units of time and length: $1 / \omega_{D}$ and $d$ (with $d$ the radius of the outer boundary or the major axis of the ellipsoid in case of oblate containers), respectively, the dimensionless equations of motion read:

$$
\begin{array}{lr}
\frac{\partial \boldsymbol{u}}{\partial t}+(\boldsymbol{u} \cdot \nabla) \boldsymbol{u}+2(\hat{\boldsymbol{z}}+\boldsymbol{\Omega}) \times \boldsymbol{u}=E \nabla^{2} \boldsymbol{u}-\nabla p_{\text {red }}-(\boldsymbol{\Omega} \times \hat{\boldsymbol{z}}) \times \boldsymbol{r} & \text { (mantle } \\
\left.\frac{\partial \boldsymbol{u}}{\partial t}+(\boldsymbol{u} \cdot \nabla) \boldsymbol{u}+2 \boldsymbol{\Omega} \times \boldsymbol{u}=E \nabla^{2} \boldsymbol{u}-\nabla p_{\text {red }}\right)
\end{array}
$$

where hats denote unit vectors and $\Omega=\Omega_{p} / \omega_{D}$, while the Ekman number is defined as: $E=\nu \cdot\left(\omega_{D} d^{2}\right)^{-1}$. 


\subsubsection{Poincaré solution}

One of the first attempts to study the problem of precession-driven flows in ellipsoidal cavities dates back to Poincaré (1910). He found that flows of uniform vorticity are solutions of the inviscid equation of motion. Consider a flow which depends linearly on the Cartesian coordinates $(x, y, z)$ such that can be considered a solid body rotation in a spherical domain. To make such a flow fit into an ellipsoid described by the equation:

$$
\frac{\tilde{x}^{2}}{a^{2}}+\frac{\tilde{y}^{2}}{a^{2}}+\frac{\tilde{z}^{2}}{c^{2}}=1
$$

stretch the coordinates and velocities as follows:

$$
\begin{array}{ccc}
x=\frac{\tilde{x}}{a} & y=\frac{\tilde{y}}{a} \quad z=\frac{\tilde{z}}{c} \\
u_{x}=\frac{\tilde{u}_{x}}{a} & u_{y}=\frac{\tilde{u}_{y}}{a} & u_{z}=\frac{\tilde{u}_{z}}{c} \\
\frac{\partial}{\partial x}=a \frac{\partial}{\partial \tilde{x}} & \frac{\partial}{\partial y}=a \frac{\partial}{\partial \tilde{y}} & \frac{\partial}{\partial z}=c \frac{\partial}{\partial \tilde{z}}
\end{array}
$$

(where the symbols with tilde describe the coordinate in an ellipsoidal domain), therefore having the following Poincaré velocity field:

$$
\begin{aligned}
& \tilde{u}_{x}=\omega_{y} \frac{a}{c} \tilde{z}-\omega_{z} \tilde{y} \\
& \tilde{u}_{y}=-\omega_{x} \frac{a}{c} \tilde{z}+\omega_{z} \tilde{x} \\
& \tilde{u}_{z}=\omega_{x} \frac{c}{a} \tilde{y}-\omega_{y} \frac{c}{a} \tilde{x}
\end{aligned}
$$

The vector $\tilde{\boldsymbol{u}}$ is solenoidal but it satisfies neither no-slip nor stress free boundary conditions and can be used consequently only as solution of the inviscid equation of motion. These flows have spatially constant vorticity and the rotation of the fluid $\boldsymbol{\omega}_{F}$ is given by:

$$
\boldsymbol{\omega}_{F}=\frac{1}{2}(\tilde{\nabla} \times \tilde{\boldsymbol{u}})=\frac{1}{2}\left[\left(\frac{a}{c}+\frac{c}{a}\right) \omega_{x},\left(\frac{a}{c}+\frac{c}{a}\right) \omega_{y}, 2 \omega_{z}\right]
$$

Taking the curl of (2.6) with $E=0$ and writing:

$\nabla \times[(\tilde{\boldsymbol{u}} \cdot \tilde{\nabla}) \tilde{\boldsymbol{u}}]=-\tilde{\nabla} \times[\tilde{\boldsymbol{u}} \times(\tilde{\nabla} \times \tilde{\boldsymbol{u}})]$, the time evolution of $\boldsymbol{\omega}_{F}$ becomes:

$$
\frac{\partial}{\partial t} \boldsymbol{\omega}_{F}-\tilde{\nabla} \times\left[\tilde{\boldsymbol{u}} \times \boldsymbol{\omega}_{F}\right]+\tilde{\nabla} \times(\boldsymbol{\Omega} \times \tilde{\boldsymbol{u}})=0
$$

Since the ellipsoid is assumed symmetric about the $z$-axis, we can choose a coordinate system in which $\Omega_{y}=0$, so that (2.10) reads:

$$
\frac{\partial}{\partial t} \omega_{F x}-\frac{a^{2}-c^{2}}{a^{2}+c^{2}} \omega_{F z} \omega_{F y}-\frac{2 a^{2}}{a^{2}+c^{2}} \omega_{F y} \Omega_{z}=0
$$




$$
\begin{gathered}
\frac{\partial}{\partial t} \omega_{F y}+\frac{a^{2}-c^{2}}{a^{2}+c^{2}} \omega_{F z} \omega_{F x}-\omega_{F z} \Omega_{x}+\frac{2 a^{2}}{a^{2}+c^{2}} \omega_{F x} \Omega_{z}=0 \\
\frac{\partial}{\partial t} \omega_{F z}+\frac{2 c^{2}}{a^{2}+c^{2}} \omega_{F y} \Omega_{x}=0
\end{gathered}
$$

In the steady case $\left(\partial \omega_{F} / \partial t=0\right)$, the third equation of the set above implies: $\omega_{F y}=0$ $\left(\Omega_{x}=0\right.$ would describe the trivial situation of no precession at all). This means that $\hat{\boldsymbol{z}}$, $\boldsymbol{\Omega}$ and $\boldsymbol{\omega}_{F}$ all lie in the same plane. The first equation of (2.11) is then automatically fullfilled, while from the second follows:

$$
\omega_{F x}\left(\frac{a^{2}-c^{2}}{a^{2}+c^{2}} \omega_{F z}+\frac{2 a^{2}}{a^{2}+c^{2}} \Omega_{z}\right)=\omega_{F z} \Omega_{x}
$$

For any $\omega_{F z}$ different from $-\frac{2 a^{2}}{a^{2}-c^{2}} \Omega_{z}$, an $\omega_{F x}$ can be found satisfying (2.12). Viscous boundary layers have to be introduced in order to select a solution (Roberts \& Stewartson, 1965; Busse, 1968).

Without precession, the fluid rotates uniformly like a solid body about the rotation axis of the container. Let us assume that at $t=0$ the precessing motion of the container is started from a state of uniform rotation. Viscous and pressure forces exerted by the boundaries will act to align the vorticity of the fluid with the rotation axis of the container, therefore at early times of the evolution $\omega_{F z} \simeq 1$ and $\omega_{F x}, \omega_{F y}=O(\boldsymbol{\Omega})$. The $z$-component of (2.11) gives:

$$
\omega_{F z} \simeq 1+O\left(\Omega^{2}\right)
$$

At low precession rates, we can guess (and numerical simulations confirm this intuition) that the fluid axis will be only slightly displaced from the container axis and at the steady case we can apply with confidence the same scaling (2.13) derived immediately after the beginning of the precessing motion, because in both cases the deviations from the solid body rotation will be small. Therefore, in this limit, $\omega_{F x}$ can be computed at first order in $\Omega$ by putting $\omega_{F z}=1$ in (2.12).

Let us now derive the angle $\vartheta$, between the fluid and the container axes (see figure 2.1), predicted by the formula (2.12).

The modulus of the rotation vector of the fluid is:

$$
\omega_{F}^{2}=\omega_{F x}^{2}+\omega_{F y}^{2}+\omega_{F z}^{2}=\omega_{F x}^{2}+1
$$

at first order in $\Omega$. Using the definitions reported in figure 2.1, it follows:

$$
\vartheta=\arccos \left(\frac{\omega_{F z}}{\omega_{F}}\right)=\arccos \left(\frac{1}{\sqrt{1+\omega_{F x}^{2}}}\right)
$$

where:

$$
\omega_{F x}=\frac{\Omega_{x}}{\frac{a^{2}-c^{2}}{a^{2}+c^{2}}+\frac{2 a^{2}}{a^{2}+c^{2}} \Omega_{z}}=\frac{\Omega_{x}}{\frac{1-(1-e)^{2}}{1+(1-e)^{2}}+\frac{2}{1+(1-e)^{2}} \Omega_{z}}
$$




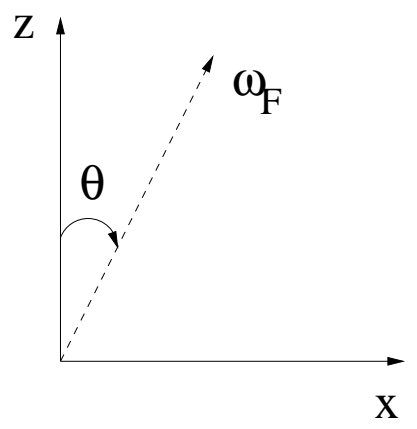

Figure 2.1: The coordinate system used in the presentation of the Poincaré solution.

and $e=1-c / a$ defines the ellipticity of the container.

\subsubsection{Busse solution}

In the previous section, it has been emphasized that the inviscid equations of motion cannot uniquely determine a solution and that Poincaré flow does not satisfy the non-slip condition at the rigid boundary. In order to select a solution, viscosity has to be taken into account. The first analytical attempt in this sense has been done by Stewartson \& Roberts (1963) and Roberts \& Stewartson (1965), who restricted the analysis to small amplitudes flows for which the boundary-layer equations can be linearized. They concluded that the boundary layer at "critical latitudes" differs from the viscous layer found elsewhere, but the interior flow maintains a constant vorticity motion.

Busse (1968) extended this linear boundary-layer analysis to include effects due to the non-linear terms in the boundary-layer equation. Under the following assumptions:

- the viscosity is small and hence the viscous term can be neglected outside a thin layer close to the boundary;

- the amplitudes of the boundary-layer flow are small;

- the ellipticity is small compared with one;

which led to a double expansion in Ekman and Rossby numbers, he derived that the main consequence of the non-linear advection of the boundary layer velocity field is a differential rotation induced in the interior of the fluid.

This second order (with respect to the Rossby number) correction contains a singularity in the limit of zero Ekman number due to the divergency of the boundary-layer thickness and corresponds to a mean flow shear layer emanating from the critical latitudes throughout the fluid coaxial with the rotation axis of the fluid. Hence the Busse solution does not approach the constant vorticity Poincare solution in the limit of vanishing viscosity. For this reason the precessional problem which involves a variation in the direction of the rotation vector of the container differs substantially from the so-called spin-up problem in which only the rate of rotation is changed. 
Unlike this latter case in which the finite-amplitude results differ only quantitatively from the results given by the linear theory, qualitatively new effects are introduced in the precessional problem taking into account the non-linear terms. The Busse formula for the fluid orientation in a spheroidal cavity of ellipticity $e$ is given (in the precession frame) by:

$$
\frac{\boldsymbol{\omega}_{F}}{\omega_{F}^{2}}=\hat{\boldsymbol{z}}+\frac{A \hat{\boldsymbol{z}} \times(\boldsymbol{\Omega} \times \hat{\boldsymbol{z}})+B(\hat{\boldsymbol{z}} \times \boldsymbol{\Omega})}{A^{2}+B^{2}}
$$

where:

$$
\begin{gathered}
A=0.259\left(E / \omega_{F}\right)^{1 / 2}+e \omega_{F}^{2}+\boldsymbol{\Omega} \cdot \hat{\boldsymbol{z}} \\
B=2.62\left(E \omega_{F}\right)^{1 / 2}
\end{gathered}
$$

with the following definitions:

$$
E=\frac{\nu}{\omega_{D} a^{2}} ; e=1-\frac{c}{a}
$$

$a$ is the major axis of the ellipsoid and $c$ is the minor axis. Rewriting $\hat{\boldsymbol{z}} \times(\boldsymbol{\Omega} \times \hat{\boldsymbol{z}})=\boldsymbol{\Omega}-\Omega_{z} \hat{\boldsymbol{z}}$ and assuming $\Omega_{y}=0$, the three components of (2.17) read:

$$
\begin{gathered}
\frac{\omega_{F x}}{\omega_{F}^{2}}=\frac{A \Omega_{x}}{A^{2}+B^{2}} \\
\frac{\omega_{F y}}{\omega_{F}^{2}}=\frac{B \Omega_{x}}{A^{2}+B^{2}} \\
\frac{\omega_{F z}}{\omega_{F}^{2}}=1
\end{gathered}
$$

The $z$-component gives: $\omega_{F}^{2}=\omega_{F z}$, which can be inserted in the other components:

$$
\begin{aligned}
& \omega_{F x}=\omega_{F z} \cdot \frac{A \Omega_{x}}{A^{2}+B^{2}} \\
& \omega_{F y}=\omega_{F z} \cdot \frac{B \Omega_{x}}{A^{2}+B^{2}}
\end{aligned}
$$

Therefore:

$$
\omega_{F z}=\omega_{F}^{2}=\omega_{F x}^{2}+\omega_{F y}^{2}+\omega_{F z}^{2}=\omega_{F z}^{2}\left[1+\frac{\Omega_{x}^{2}}{\left(A^{2}+B^{2}\right)}\right]
$$

The $\vartheta$ angle, between the fluid and the container axes (see figure 2.1), can be evaluated as follows:

$$
\vartheta=\arccos \left(\frac{\omega_{F z}}{\omega_{F}}\right)=\arccos \left(\sqrt{\omega_{F z}}\right)
$$

where $\omega_{F z}$ is the zero of (2.18). 
In a spherical cavity $(e=0)$, from (2.17) follows that the direction of $\boldsymbol{\omega}_{F}$ varies as a function of $\sqrt{E} / \Omega$ along a cone of nearly half-circular shape. The edges of the cone corresponding to the end-points of the half-circle are given by $\Omega$ and $\hat{\boldsymbol{z}}$. As the precessional rate $\Omega$ increases from zero the vector $\boldsymbol{\omega}_{F}$ is displaced from $\hat{\boldsymbol{z}}$ in the direction perpendicular to the meridional plane through $\hat{\boldsymbol{z}}$ and $\boldsymbol{\Omega}$. As $|\boldsymbol{\Omega} \cdot \hat{\boldsymbol{z}}|$ becomes large in comparison with $\sqrt{E}, \boldsymbol{\omega}_{F}$ moves on the conical surface towards the direction of $\boldsymbol{\Omega}$.

\section{$2.2 \quad$ Numerical methods}

\subsubsection{Spatial discretization}

In the present research work, the motion of an incompressible fluid of kinematic viscosity $\nu$ contained in an ellipsoidal cavity has been investigated by direct numerical simulations (DNS).

The ellipsoidal shell rotates about its axis of symmetry (along the $\hat{\boldsymbol{z}}$ direction) with angular frequency $\omega_{D}$.

Furthermore, the rotation axis is executing precessional motion characterized by the vector $\Omega_{p} \hat{\Omega}_{p}$ (hats denote unit vectors).

Considering the regularity of the geometry in the problem addressed in the following, a spectral method seems to be the obvious choice in order to obtain high accuracy and rapid convergence.

These methods, however, face the problem of a boundary geometry which is not easily accommodated by any standard coordinate system.

In order to overcome this difficulty, the ellipsoidal fluid volume has been distorted, by a coordinate transformation, into a spherical domain.

Therefore, in the following, two coordinate systems will be used: the original one, in which the boundaries are ellipsoids of revolution (described by symbols with tildes) and the computational one, in which the boundaries are spherical. The boundaries of the ellipsoidal shell are given by:

$$
\begin{gathered}
\frac{\tilde{x}^{2}}{a^{2}}+\frac{\tilde{y}^{2}}{a^{2}}+\frac{\tilde{z}^{2}}{c^{2}}=1 \\
\frac{\tilde{x}^{2}}{(\eta a)^{2}}+\frac{\tilde{y}^{2}}{(\eta a)^{2}}+\frac{\tilde{z}^{2}}{(\eta c)^{2}}=1
\end{gathered}
$$

with $\eta<1$ and both boundaries having the same ellipticity $e=1-c / a$.

Choosing $(1-\eta) a$ as unit of length and $1 / \omega_{D}$ as unit of time, the non-dimensional equations for the velocity $\tilde{\boldsymbol{u}}(\tilde{\boldsymbol{r}}, t)$ read in a frame of reference attached to the shell (mantle frame):

$$
\begin{gathered}
\frac{\partial}{\partial t} \tilde{\nabla} \times \tilde{\boldsymbol{u}}+\tilde{\nabla} \times\{(2(\hat{\tilde{\boldsymbol{z}}}+\mathbf{\Omega})+\tilde{\nabla} \times \tilde{\boldsymbol{u}}) \times \tilde{\boldsymbol{u}}\}=E \tilde{\nabla}^{2} \tilde{\nabla} \times \tilde{\boldsymbol{u}}+2 \hat{\tilde{\boldsymbol{z}}} \times \mathbf{\Omega} \\
\tilde{\nabla} \cdot \tilde{\boldsymbol{u}}=0
\end{gathered}
$$


The Ekman number $E$ is defined by $E=\nu\left(\omega_{D}(1-\eta)^{2} a^{2}\right)^{-1}$ and $\boldsymbol{\Omega}=\Omega_{p} / \omega_{D} \hat{\Omega}_{p}$.

The spherical computational domain is introduced by stretching the coordinates:

$$
x=\tilde{x} \quad y=\tilde{y} \quad z=\frac{\tilde{z}}{1-e}
$$

If the velocities are transformed likewise,

$$
u_{x}=\tilde{u}_{x} \quad u_{y}=\tilde{u}_{y} \quad u_{z}=\frac{\tilde{u}_{z}}{1-e}
$$

one obtains again a solenoidal vector field, $\nabla \cdot \boldsymbol{u}=0$.

The transformations introduced above lead to spherical boundaries which can be easily handled by spectral methods:

$$
\begin{aligned}
& x^{2}+y^{2}+z^{2}=r_{o}^{2} \\
& x^{2}+y^{2}+z^{2}=r_{i}^{2}
\end{aligned}
$$

( $r_{i}$ and $r_{o}$ denote the inner and outer radii of the spherical shell) with $r_{i} / r_{o}=\eta$ and $r_{o}-r_{i}=1$, at the price of an increased complexity for the equation of motion:

$$
\begin{array}{r}
\frac{\partial}{\partial t} \nabla \times \boldsymbol{u}-E \nabla^{2} \nabla \times \boldsymbol{u}+\boldsymbol{L} \\
=-\nabla \times \boldsymbol{N}+e \nabla \times\left(N_{z} \hat{\boldsymbol{z}}\right)+2(1-e) \hat{\boldsymbol{z}} \times \boldsymbol{\Omega}
\end{array}
$$

The nonlinear and Coriolis terms have been grouped together as well as the linear terms which vanish for $e=0$ :

$$
\begin{gathered}
\boldsymbol{N}=(2(\hat{\tilde{\boldsymbol{z}}}+\mathbf{\Omega})+\tilde{\nabla} \times \tilde{\boldsymbol{u}}) \times \tilde{\boldsymbol{u}} \\
\boldsymbol{L}=e(e-2) \frac{\partial}{\partial t} \nabla \times\left(u_{z} \hat{\boldsymbol{z}}\right)-E \frac{e(2-e)}{(1-e)^{2}} \frac{\partial^{2}}{\partial z^{2}} \nabla \times \boldsymbol{u} \\
-E\left[e(e-2) \nabla^{2}-\left(\frac{e(e-2)}{1-e}\right)^{2} \frac{\partial^{2}}{\partial z^{2}}\right] \nabla \times\left(u_{z} \hat{\boldsymbol{z}}\right)
\end{gathered}
$$

The precession axis $\hat{\boldsymbol{\Omega}}$ forms the angle $\alpha(0<\alpha<\pi / 2)$ with the $z$-axis and is time dependent in the chosen system of reference:

$$
\hat{\boldsymbol{\Omega}}=\sin \alpha \cos t \hat{\boldsymbol{x}}-\sin \alpha \sin t \hat{\boldsymbol{y}}+\cos \alpha \hat{\boldsymbol{z}}
$$

Retrograde precession corresponds to $\Omega<0$.

The no-slip boundary conditions require that $\boldsymbol{u}=0$ at $r=r_{i}, r_{o}$.

The solenoidal vector field $\boldsymbol{u}$ can be written in terms of poloidal and toroidal scalars $\Phi$ and $\Psi$ : 


$$
\boldsymbol{u}=\nabla \times \nabla \times(\Phi \hat{\boldsymbol{r}})+\nabla \times(\Psi \hat{\boldsymbol{r}})
$$

which are then decomposed in spherical harmonics:

$$
\begin{gathered}
\Phi=r \sum_{l=1}^{\infty} \sum_{m=-l}^{l} V_{l}^{m}(r, t) P_{l}^{m}(\cos \vartheta) e^{i m \varphi} \\
\Psi=r^{2} \sum_{l=1}^{\infty} \sum_{m=-l}^{l} W_{l}^{m}(r, t) P_{l}^{m}(\cos \vartheta) e^{i m \varphi}
\end{gathered}
$$

$P_{l}^{m}(\cos \vartheta)$ denote associated Legendre functions.

The factors $r$ and $r^{2}$ in (2.33) are not essential but convenient because they reduce the variations of $V_{l}^{m}$ and $W_{l}^{m}$ with $r$.

The three components of the velocity and vorticity fields can then be written as follows:

$$
\begin{aligned}
& u_{r}=\sum_{m=-\infty}^{\infty} \sum_{l=|m|}^{\infty} \frac{l(l+1)}{r} V_{l}^{m}(r) P_{l}^{m}(\cos \vartheta) e^{i m \varphi} \\
& u_{\vartheta}=\sum_{m=-\infty}^{\infty} \sum_{l=|m|}^{\infty}\left(\frac{1}{r} V_{l}^{m}(r)+\frac{\partial}{\partial r} V_{l}^{m}(r)\right) \frac{d}{d \vartheta} P_{l}^{m}(\cos \vartheta) e^{i m \varphi}+ \\
& \frac{i m r}{\sin \vartheta} W_{l}^{m}(r) P_{l}^{m}(\cos \vartheta) e^{i m \varphi} \\
& u_{\varphi}=\sum_{m=-\infty}^{\infty} \sum_{l=|m|}^{\infty} \frac{i m}{\sin \vartheta}\left(\frac{1}{r} V_{l}^{m}(r)+\frac{\partial}{\partial r} V_{l}^{m}(r)\right) P_{l}^{m}(\cos \vartheta) e^{i m \varphi}- \\
& r W_{l}^{m}(r) \frac{d}{d \vartheta} P_{l}^{m}(\cos \vartheta) e^{i m \varphi} \\
& (\nabla \times \boldsymbol{u})_{r}=\sum_{m=-\infty}^{\infty} \sum_{l=|m|}^{\infty} l(l+1) W_{l}^{m}(r) P_{l}^{m}(\cos \vartheta) e^{i m \varphi} \\
& (\nabla \times \boldsymbol{u})_{\vartheta}=\sum_{m=-\infty}^{\infty} \sum_{l=|m|}^{\infty}\left(2 W_{l}^{m}(r)+r \frac{\partial}{\partial r} W_{l}^{m}(r)\right) \frac{d}{d \vartheta} P_{l}^{m}(\cos \vartheta) e^{i m \varphi}+ \\
& \frac{i m}{r \sin \vartheta}\left(\frac{l(l+1)}{r} V_{l}^{m}(r)-2 \frac{\partial}{\partial r} V_{l}^{m}(r)-r \frac{\partial^{2}}{\partial r^{2}} V_{l}^{m}(r)\right) \text {. } \\
& P_{l}^{m}(\cos \vartheta) e^{i m \varphi} \\
& (\nabla \times \boldsymbol{u})_{\varphi}=\sum_{m=-\infty}^{\infty} \sum_{l=|m|}^{\infty} \frac{i m}{\sin \vartheta}\left(2 W_{l}^{m}(r)+r \frac{\partial}{\partial r} W_{l}^{m}(r)\right) . \\
& P_{l}^{m}(\cos \vartheta) e^{i m \varphi}-\frac{1}{r}\left(\frac{l(l+1)}{r} V_{l}^{m}(r)-2 \frac{\partial}{\partial r} V_{l}^{m}(r)-\right. \\
& \left.r \frac{\partial^{2}}{\partial r^{2}} V_{l}^{m}(r)\right) \frac{d}{d \vartheta} P_{l}^{m}(\cos \vartheta) e^{i m \varphi}
\end{aligned}
$$


Operating with $\hat{\boldsymbol{r}}$. and $\hat{\boldsymbol{r}} \cdot \nabla \times$ on $(2.28)$ one obtains two equations for $V_{l}^{m}(r, t)$ and $W_{l}^{m}(r, t)$ :

$$
\begin{gathered}
\frac{\partial}{\partial t} \mathcal{D}_{l} V_{l}^{m}-E \cdot \mathcal{D}_{l}^{2} V_{l}^{m}-\frac{r}{l(l+1)}[\hat{\boldsymbol{r}} \cdot \nabla \times \boldsymbol{L}]_{l}^{m}= \\
\frac{r}{l(l+1)}\left[\hat{\boldsymbol{r}} \cdot \nabla \times\left\{\nabla \times \boldsymbol{N}-e \nabla \times\left(N_{z} \hat{\boldsymbol{z}}\right)\right\}\right]_{l}^{m} \\
\frac{\partial}{\partial t} W_{l}^{m}-E\left(\frac{\partial^{2}}{\partial r^{2}}+\frac{4}{r} \frac{\partial}{\partial r}+\frac{2-l(l+1)}{r^{2}}\right) W_{l}^{m}+\frac{1}{l(l+1)}[\hat{\boldsymbol{r}} \cdot \boldsymbol{L}]_{l}^{m}= \\
-\frac{1}{l(l+1)}\left[\hat{\boldsymbol{r}} \cdot\left\{\nabla \times \boldsymbol{N}-e \nabla \times\left(N_{z} \hat{\boldsymbol{z}}\right)\right\}\right]_{l}^{m}+(1-e)[f]_{l}^{m}
\end{gathered}
$$

with:

$$
\begin{gathered}
\mathcal{D}_{l}=\frac{\partial^{2}}{\partial r^{2}}+\frac{2}{r} \frac{\partial}{\partial r}-\frac{l(l+1)}{r^{2}} \\
f=\frac{1}{2} \Omega \sin \alpha\left[i P_{1}^{1} e^{i(\varphi+t)}+c . c .\right]
\end{gathered}
$$

[]$_{l}^{m}$ denotes the $l, m$-component of the quantity in the square bracket.

The no-slip boundary conditions become:

$$
V_{l}^{m}=\frac{\partial V_{l}^{m}}{\partial r}=W_{l}^{m}=0 \text { at } r=r_{i}, r_{o}
$$

Because $\Phi$ and $\Psi$ are real, $V_{l}^{m *}=(-1)^{m} \frac{(l-m) !}{(l+m) !} V_{l}^{-m}$ and $W_{l}^{m *}=(-1)^{m} \frac{(l-m) !}{(l+m) !} W_{l}^{-m}$ so that only components with $m \geq 0$ need to be stored. The sums in (2.33) are truncated to include only terms with $l \leq L$ and consequently $-L \leq m \leq L$.

Equations (2.34), (2.35) are solved with a Chebychev collocation method, therefore the functions $V_{l}^{m}$ and $W_{l}^{m}$ are expanded in $N_{r}$ Chebychev polynomials $T_{n}$ as:

$$
V_{l}^{m}(r, t)=\sum_{n=0}^{N_{r}-1} v_{l, n}^{m}(t) T_{n}(x) \quad, \quad W_{l}^{m}(r, t)=\sum_{n=0}^{N_{r}-1} w_{l, n}^{m}(t) T_{n}(x)
$$

with $x=2\left(r-r_{i}\right)-1$.

The collocation points are placed in direct space at:

$$
r_{j}=r_{i}+\frac{1}{2}\left(1+\cos \pi \frac{j-1}{N_{r}-1}\right) \quad \text { with } \mathrm{j}=1, \cdots, \mathrm{N}_{\mathrm{r}}
$$

so that a fast cosine transform can be used to switch between physical and spectral space.

This follows immediately from the fact that the Chebychev polynomials can be represented in terms of trigonometric functions as:

$$
T_{n}(x)=\cos (n \arccos x)
$$


and therefore, with the definition of collocation points given above:

$$
T_{n}(x)=\cos \left(n \pi \frac{j-1}{N_{r}-1}\right)
$$

Inserting this last expression in the first of (2.37), for example, gives:

$$
V_{l}^{m}\left(r_{j}, t\right)=\sum_{n=0}^{N_{r}-1} v_{l, n}^{m}(t) \cos \left(n \pi \frac{j-1}{N_{r}-1}\right) \quad j=1, \cdots, N_{r}
$$

which is a cosine transform.

Equations (2.34), (2.35) are enforced at every collocation point and the spectral representation in radius is only used to compute derivatives.

In order to calculate the nonlinear terms which, in the computational domain, are written as:

$$
\begin{aligned}
\mathbf{N}= & 2 \hat{\boldsymbol{z}} \times \boldsymbol{u}+\left[\left(\begin{array}{c}
2 \Omega_{x} \\
2 \Omega_{y} \\
2 \Omega_{z}
\end{array}\right)+\left(\begin{array}{c}
\frac{1}{(1-e)}(\nabla \times \boldsymbol{u})_{x} \\
\frac{1}{(1-e)}(\nabla \times \boldsymbol{u})_{y} \\
(\nabla \times \boldsymbol{u})_{z}
\end{array}\right)+\frac{e(e-2)}{(1-e)} \nabla \times\left(\begin{array}{c}
0 \\
0 \\
u_{z}
\end{array}\right)\right] \times \\
& \left(\begin{array}{c}
u_{x} \\
u_{y} \\
(1-e) u_{z}
\end{array}\right)
\end{aligned}
$$

$\boldsymbol{u}, \nabla \times \boldsymbol{u}$ and $\nabla \times\left(u_{z} \hat{\boldsymbol{z}}\right)$ are firstly evaluated in $(r, \vartheta, \varphi)$-space (see Appendix A for details) and then converted into $(x, y, z)$-components so that equations $(2.25)$ can be easily applied.

The result is transformed back into $(r, l, m)$-space.

In general a function $f(r, \vartheta, \varphi)$ is transformed into its coefficients $[f]_{l}^{m}(r)$ and back using the formulas:

$$
\begin{gathered}
f(r, \vartheta, \varphi)=\sum_{m=-\infty}^{\infty} \sum_{l=|m|}^{\infty}[f]_{l}^{m}(r) P_{l}^{m}(\cos \vartheta) e^{i m \varphi} \\
{[f]_{l}^{m}(r)=(-1)^{m} \frac{(2 l+1)}{4 \pi} \int_{-1}^{1} d x P_{l}^{-m}(x) \int_{0}^{2 \pi} d \varphi e^{-i m \varphi} f(r, \arccos x, \varphi), \quad x=\cos \vartheta}
\end{gathered}
$$

The transformation $(l, m) \rightarrow(\vartheta, \varphi)$ requires a weighted sum of associated Legendre functions (a matrix-vector multiplication) followed by a Fast Fourier Transform (FFT) over the index $m$.

The inverse transformation starts with the FFT and then needs to compute the projection integral. This is done with a Gaussian quadrature which approximates the integral over $x$ by the sum:

$$
\int_{-1}^{1} g(x) d x=\sum_{i=1}^{L} w_{i} g\left(x_{i}\right)
$$


The approximation given by the last equation is exact if $g$ is a polynomial in $x$ of order $2 L+1$ or less. The non-linear terms, with toroidal and poloidal scalars expanded in spherical harmonics indeed yield polynomials in $\cos \vartheta$.

Evaluation of the right hand sides of equations (2.34), (2.35) requires the calculation of the radial component of the curl and the radial component of the curl of the curl of a vector $\boldsymbol{S}=S_{r} \hat{\boldsymbol{r}}+S_{\vartheta} \hat{\boldsymbol{\vartheta}}+S_{\varphi} \hat{\boldsymbol{\varphi}}$.

Following Glatzmaier (1984) and Young (1974), the auxiliary quantities $P_{r}=r^{2} S_{r}$, $P_{\vartheta}=S_{\vartheta} /(r \sin \vartheta)$ and $P_{\varphi}=S_{\varphi} /(r \sin \vartheta)$ are introduced, calculated in direct space and finally transformed into $(r, l, m)$-space.

In this way the derivatives of the vector $\boldsymbol{S}$ can be easily obtained:

$$
\begin{gathered}
{[\hat{\boldsymbol{r}} \cdot \nabla \times \boldsymbol{S}]_{l}^{m}=(l+1) \frac{(l-m)}{(2 l-1)}\left[P_{\varphi}\right]_{l-1}^{m}-l \frac{(l+m+1)}{(2 l+3)}\left[P_{\varphi}\right]_{l+1}^{m}-i m\left[P_{\vartheta}\right]_{l}^{m}} \\
{[\hat{\boldsymbol{r}} \cdot \nabla \times \nabla \times \boldsymbol{S}]_{l}^{m}=\frac{l(l+1)}{r^{4}}\left[P_{r}\right]_{l}^{m}+\frac{1}{r^{2}} \frac{d}{d r}\left[r ^ { 2 } \left((l+1) \frac{(l-m)}{(2 l-1)}\left[P_{\vartheta}\right]_{l-1}^{m}-\right.\right.} \\
\left.\left.l \frac{(l+m+1)}{(2 l+3)}\left[P_{\vartheta}\right]_{l+1}^{m}+i m\left[P_{\varphi}\right]_{l}^{m}\right)\right]
\end{gathered}
$$

The computation of the radial derivative in (2.40) requires a transformation into Chebychev space and back.

When the equations (2.39) and (2.40) are truncated at the order $L$, severe errors may arise because the components of $\boldsymbol{S}$ at order $L+1$ are not known. In order to solve this difficulty, the terms $[\hat{\boldsymbol{r}} \cdot \nabla \times \boldsymbol{S}]_{L}^{m}$ and $[\hat{\boldsymbol{r}} \cdot \nabla \times \nabla \times \boldsymbol{S}]_{L}^{m}$ were set to zero.

In this specific context:

$$
\boldsymbol{S}=\boldsymbol{N}-e N_{z} \hat{\boldsymbol{z}}
$$

where $\boldsymbol{N}$ is given by (2.38) except for the Coriolis term, $2 \hat{\boldsymbol{z}} \times \boldsymbol{u}$, which is computed directly. This is made in order to avoid the time consuming steps required for the computation of $(2.39),(2.40)$ when one deals with the linearized equations. The formulas used are:

$$
\begin{aligned}
-\frac{2}{l(l+1)}[\hat{\boldsymbol{r}} \cdot \nabla \times(\hat{\boldsymbol{z}} \times \boldsymbol{u})]_{l}^{m}= & \frac{2}{l(l+1)} \frac{1}{r^{2}}\left(\frac{l(l+1)(l+2)(l+m+1)}{(2 l+3)} \cdot V_{l+1}^{m}(r)+\right. \\
& \frac{l(l+2)(l+m+1)}{(2 l+3)}\left(V_{l+1}^{m}(r)+r \frac{\partial}{\partial r} V_{l+1}^{m}(r)\right)- \\
& \frac{l(l+1)(l-1)(l-m)}{(2 l-1)} \cdot V_{l-1}^{m}(r)+ \\
& \frac{(l+1)(l-1)(l-m)}{(2 l-1)}\left(V_{l-1}^{m}(r)+r \frac{\partial}{\partial r} V_{l-1}^{m}(r)\right)+ \\
& \left.i m r^{2} W_{l}^{m}(r)\right)
\end{aligned}
$$




$$
\begin{aligned}
\frac{2 r}{l(l+1)}[\hat{\boldsymbol{r}} \cdot \nabla \times \nabla \times(\hat{\boldsymbol{z}} \times \boldsymbol{u})]_{l}^{m}= & \frac{2}{l(l+1)}\left(-\frac{l(l+1)(l+2)(l+m+1)}{(2 l+3)} \cdot W_{l+1}^{m}(r)-\right. \\
& \frac{l(l+2)(l+m+1)}{(2 l+3)}\left(2 W_{l+1}^{m}(r)+r \frac{\partial}{\partial r} W_{l+1}^{m}(r)\right)+ \\
& \frac{l(l+1)(l-1)(l-m)}{(2 l-1)} \cdot W_{l-1}^{m}(r)- \\
& \frac{(l+1)(l-1)(l-m)}{(2 l-1)}\left(2 W_{l-1}^{m}(r)+r \frac{\partial}{\partial r} W_{l-1}^{m}(r)\right)+ \\
& \left.i m \mathcal{D}_{l} V_{l}^{m}(r)\right)
\end{aligned}
$$

In the limit of $E \rightarrow 0$, the dissipative term in the equation of motion can be neglected and the kinetic energy of the flow is conserved: $\partial / \partial t \int \frac{1}{2} \boldsymbol{u}^{2} d V=0$. The numerical method used for the discretization of the equations of the motion will be accurate and stable if the truncated system will also conserve energy. It is well known (Canuto, Hussaini, et al.) that if the basis functions of a Galerkin spectral method are mutually orthogonal, the numerical method will obey the same conservation law. The angular variables are indeed discretized with a Galerkin method, while for the radial component a collocation method has been used. Since it appears difficult to find a strictly energy conserving method based on Chebychev polynomials, in the scheme adopted here the quality of energy conservation is expected to depend on radial resolution (see [72] for a detailed description of this issue).

\subsubsection{Temporal discretization}

The equations (2.34), (2.35) are discretized in time using implicit Euler steps (which can procure higher "fidelity" than, for example, the Crank-Nicolson step) for the implicit part (linear terms) and second order Adams-Bashforth steps for the explicit part (nonlinear terms) [see [72] for a detailed discussion about the convenience of this choice].

If $y(t)$ is a vector containing the coefficients $v_{l, n}^{m}(t)$ and $w_{l, n}^{m}(t)$, the discretized equations of motion can be written for a time step of size $h$ in the form:

$$
\boldsymbol{M} \boldsymbol{y}(t+h)=\boldsymbol{M}^{\prime} \boldsymbol{y}(t)+\frac{h}{2}[3 \boldsymbol{n l}(\boldsymbol{y}(t))-\boldsymbol{n l}(\boldsymbol{y}(t-h))]
$$

where $\boldsymbol{M}$ and $\boldsymbol{M}^{\prime}$ represent matrices and $\boldsymbol{n} \boldsymbol{l}$ all nonlinear terms and the Coriolis force. For the implicit part of the time step, linear systems of the form

$$
\boldsymbol{M}_{1} \boldsymbol{y}+\boldsymbol{M}_{2} \boldsymbol{y}=\boldsymbol{A}
$$

need to be solved, where $\boldsymbol{M}_{1}$ represents the discretization of the first two terms in (2.28), $\boldsymbol{M}_{2}$ the remaining terms of the left hand side of (2.28), and $\boldsymbol{A}$ the right hand side of (2.43). $\boldsymbol{M}_{1}$ decouples in $l$ and $m$ and therefore consists of small blocks which are even independent of $m$. For the toroidal components, the coefficient matrices $C^{l}$ have been constructed such that: 


$$
\sum_{j=1}^{n_{r}} C_{n, j}^{l} w_{l, j}^{m}=\sum_{j=1}^{n_{r}} w_{l, j}^{m}\left(T_{j-1}\left(x_{n}\right)-h E\left(\frac{\partial^{2}}{\partial r^{2}}+\frac{4}{r} \frac{\partial}{\partial r}+\frac{2-l(l+1)}{r^{2}}\right) T_{j-1}\left(x_{n}\right)\right)
$$

with: $n=2, \cdots, n_{r}-1, l=1, \cdots, L$.

Boundary conditions are enforced by using the equations corresponding to the boundary points:

$$
C_{1, j}^{l}=1, \quad C_{n_{r}, j}^{l}=(-1)^{j-1}, \quad j=1, \cdots, n_{r}, \quad l=1, \cdots, L
$$

Likewise for the poloidal components the matrices $C^{\prime l}$ have been computed as follows:

$$
\sum_{j=1}^{n_{r}} C_{n, j}^{\prime l} v_{l, j}^{m}=\sum_{j=1}^{n_{r}} v_{l, j}^{m}\left(\mathcal{D}_{l} T_{j-1}\left(x_{n}\right)-h E \mathcal{D}_{l}^{2} T_{j-1}\left(x_{n}\right)\right), \quad n=3, \cdots, n_{r}-2, \quad l=1, \cdots, L
$$

with boundary conditions enforced at two more collocations points:

$$
\begin{gathered}
C_{1, j}^{\prime l}=1, \quad C_{n_{r}, j}^{\prime l}=(-1)^{j-1}, \quad j=1, \cdots, n_{r} \\
C_{2, j}^{\prime l}=(j-1)^{2}, \quad C_{n_{r}-1, j}^{\prime l}=(-1)^{j}(j-1)^{2}, \quad j=1, \cdots, n_{r}
\end{gathered}
$$

The matrices $\boldsymbol{C}^{l}$ and $\boldsymbol{C}^{\prime l}$ are computed, inverted and stored for later use during an initialization step.

In order to solve the full system, a Jacobi type iteration is employed:

$$
\boldsymbol{y}_{n+1}=\boldsymbol{M}_{1}^{-1}\left(-\boldsymbol{M}_{2} \boldsymbol{y}_{n}+\boldsymbol{A}\right)
$$

where $\boldsymbol{y}_{n}$ is the $n$-th iterate of the solution.

The Jacobi method (among the more general 'relaxation methods') is usually considered in literature of academic interest and of no practical relevance because it converges too slowly. In spite of that, a successful implementation has been realized for the problem addressed here, although the number of iterations necessary to achieve satisfactory accuracy increases with increasing $e$. In the present application, only small ellipticities are of interest and about 5 iterations are enough to reach convergence so that it did not seem useful to opt for a more powerful method. For $e>0.2$ however, the Jacobi iteration diverges (see Appendix A for a more detailed description).

$\boldsymbol{M}_{2}$ contains a multitude of terms in which $\hat{\boldsymbol{r}} \cdot \nabla \times$ and $\hat{\boldsymbol{r}} \cdot \nabla \times \nabla \times$ are applied to expressions in which $u_{z} \hat{z}$ or second derivatives $\frac{\partial^{2}}{\partial z^{2}}$ occur, therefore this matrix still decouples in $m$ but couples different values of $l$. It would require a large amount of storage to keep $\boldsymbol{M}_{2}$ in memory at any one time so that the effect of $\boldsymbol{M}_{2}$ on $\boldsymbol{y}$ is encoded in a set of subroutines instead, used to compute also $\boldsymbol{A}$ (see Appendix A for a detailed description of the practical implementation).

The CPU intensive procedures at high resolutions are those involving matrix-vector multiplications, i.e. the implicit time step and the $l \rightarrow \vartheta$ transformations. The dominant 
contribution per time step is, therefore, $N_{r}^{2} L M$ or $N_{r} L^{2} M$, depending on which of the operations above mentioned is larger. If the grid contains $N$ points in all directions, this amounts to $N^{4}$. At the typical resolutions used here $\left(N_{r}=129, L=128, M=64\right)$, the operation load scales somewhere in between $N^{3}$ and $N^{4}$. All steps of the program execute in parallel (see Appendix A for details).

Apart from the special case $e=0$, nothing seems to be available to comprehensively validate the ellipsoidal code. The program had to be tested term by term by comparing numerical output with calculations made by hand for representative examples of poloidal and toroidal scalars. However, some validation is provided by the agreement between the computed laminar zonal flow and the experimental observations and by the comparison with the asymptotic theory of Busse (1968).

\subsection{Laminar flows}

The Poincaré solution for inviscid fluids is a solid-body rotation described by:

$$
\boldsymbol{u}=\boldsymbol{\omega} \times \boldsymbol{r} \quad \text { (in spherical coordinates) }
$$

where $\boldsymbol{\omega}$ is a constant vector. When the viscosity is different from zero but still small, we expect deviations from a solution of uniform vorticity particularly meaningful in a thin region close to the boundary of the container.

In order to quantify the effects of viscosity, it is therefore useful to consider a local rotation vector depending on the radial coordinate which corresponds to the solid body rotation component of the motion of the fluid at radius $r$ :

$$
\boldsymbol{\omega}(r)=\left(1-e+\frac{1}{1-e}\right)\left[-\Re_{e}\left\{W_{1}^{1}(r)\right\} \hat{\boldsymbol{x}}+\Im_{m}\left\{W_{1}^{1}(r)\right\} \hat{\boldsymbol{y}}\right]+W_{1}^{o}(r) \hat{\boldsymbol{z}}+\hat{\boldsymbol{z}}
$$

The orientation of the numerically determined rotation vector $\boldsymbol{\omega}_{F}$, calculated as average of $\boldsymbol{\omega}(r)$ over the fluid volume, can then be compared with the Busse solution reported in section (2.1.2). This comparison is made in table 2.1.

Even at small angles between $\boldsymbol{\omega}_{F}$ and $\hat{\boldsymbol{z}}$ (that means small values of $|\boldsymbol{\Omega}|$ ) differences between the Busse formula and the numerical results should be expected since the effect of the divergence of the Ekman layer at the critical latitudes is only approximately taken into account in the analytical theory.

The numerical results show significant deviations from a solid body rotation in the interior of the flow, assumed as a basis for the derivation of the analytical expression $(2.17)$.

In spite of that, the analytical and numerical results agree well also beyond the approximate limits of validity of the analytical formula.

However, significant differences start to appear as $|\boldsymbol{\Omega}|$ increases and the ellipticity of the container $e$ is not anymore small (see cases with $e=0.1$ and $|\boldsymbol{\Omega}|=-8 \cdot 10^{-2}$ in table $2.1)$. 


\begin{tabular}{|c|c|c|c|c|c|}
\hline$E$ & $e$ & $\alpha$ & $\Omega$ & $\theta_{F}$ & $\varphi_{F}$ \\
\hline $10^{-4}$ & 0.04 & $30^{\circ}$ & $-10^{-5}$ & $5.59^{o} \times 10^{-3}\left(5.73^{o} \times 10^{-3}\right)$ & $-149.76^{\circ}\left(-148.40^{\circ}\right)$ \\
\hline $10^{-5}$ & 0.04 & $30^{\circ}$ & $-10^{-5}$ & $6.70^{\circ} \times 10^{-3}\left(6.88^{\circ} \times 10^{-3}\right)$ & $-169.47^{\circ}\left(-168.52^{\circ}\right)$ \\
\hline $10^{-6}$ & 0.04 & $30^{\circ}$ & $-10^{-5}$ & $6.77^{o} \times 10^{-3}\left(7.11^{o} \times 10^{-3}\right)$ & $-176.76^{\circ}\left(-176.27^{\circ}\right)$ \\
\hline $10^{-5}$ & 0 & $30^{\circ}$ & $-10^{-5}$ & $3.65^{o} \times 10^{-2}\left(3.44^{o} \times 10^{-2}\right)$ & $-104.51^{o}\left(-95.59^{\circ}\right)$ \\
\hline $10^{-4}$ & 0.04 & $30^{\circ}$ & $-10^{-3}$ & $0.568^{\circ}\left(0.581^{o}\right)$ & $-149.25^{\circ}\left(-147.87^{\circ}\right)$ \\
\hline $10^{-4}$ & 0.04 & $30^{\circ}$ & $-10^{-2}$ & $6.62^{\circ}\left(6.73^{\circ}\right)$ & $-143.77^{\circ}\left(-141.97^{\circ}\right)$ \\
\hline $10^{-4}$ & 0.04 & $30^{\circ}$ & -0.03 & $31.31^{\circ}\left(30.90^{\circ}\right)$ & $-103.44^{o}\left(-104.48^{o}\right)$ \\
\hline $10^{-4}$ & 0.04 & $30^{\circ}$ & -0.1 & $36.74^{\circ}\left(38.25^{\circ}\right)$ & $-18.38^{\circ}\left(-21.48^{\circ}\right)$ \\
\hline $10^{-4}$ & 0.04 & $30^{\circ}$ & -0.2 & $32.14^{o}\left(34.56^{o}\right)$ & $-7.57^{\circ}\left(-9.43^{\circ}\right)$ \\
\hline $5 \times 10^{-4}$ & 0.1 & $80^{\circ}$ & -0.08 & $53.98^{\circ}\left(59.69^{\circ}\right)$ & $-104.38^{\circ}\left(-115.37^{\circ}\right)$ \\
\hline $10^{-4}$ & 0.1 & $80^{\circ}$ & -0.08 & $74.79^{\circ}\left(82.10^{\circ}\right)$ & $-72.79^{\circ}\left(-62.69^{\circ}\right)$ \\
\hline $2 \times 10^{-5}$ & 0.04 & $30^{\circ}$ & -0.018 & $19.96^{\circ}\left(21.06^{\circ}\right)$ & $-153.68^{\circ}\left(-151.03^{\circ}\right)$ \\
\hline
\end{tabular}

Table 2.1: $\theta_{F}$ and $\varphi_{F}$ give for some representative cases the orientation of $\boldsymbol{\omega}_{F}$ in polar angles. The precession axis is located in the plane $\varphi=0$. Numbers in brackets are predictions from asymptotic theory. All runs are with $\eta=0.1$.

In the ellipsoid the agreement is of the same quality as found in spherical geometry by Tilgner \& Busse (2001). The detailed variations of $\boldsymbol{\omega}(r)$ for low precession rates are summarized in figure 2.2.

Except for the expected rapid variation in the Ekman boundary layer, at constant ellipticity, the solution throughout the interior of the fluid approaches a state of uniform vorticity as $E$ decreases.

At the same time, the orientation of $\boldsymbol{\omega}_{F}$ progressively rotates into the $(\hat{\boldsymbol{z}}, \hat{\boldsymbol{\Omega}})$-plane.

In a sphere, characterized by the property that the effect of the pressure vanishes leaving the viscosity as the sole efficient mechanism to couple fluid and container, $\boldsymbol{\omega}_{F}$ lies nearly perpendicular to the $(\hat{\boldsymbol{z}}, \hat{\boldsymbol{\Omega}})$-plane [see table 2.1 and Tilgner \& Busse (2001)].

As $E$ is reduced in an elliptical container, viscous forces become smaller compared with pressure forces, so that one goes continuously from the orientation typical in spherical geometry to the Poincaré solution for inviscid flow in which $\boldsymbol{\omega}_{F}$ lies in the $(\hat{\boldsymbol{z}}, \hat{\boldsymbol{\Omega}})$-plane.

The absolute value of $\boldsymbol{\omega}(r)$ (equation (2.47)) remains almost constant (in the interior of the flow) as a function of $r$ if $E$ is not too small, as shown in figure 2.2 (bottom). The interior values are somewhat higher than the value obtained from (2.17).

When $E$ decreases the spin-up process driven by the Ekman suction becomes inefficient and $\boldsymbol{\omega}(r)$ decreases towards the centre as indicated by the solution obtained for $E=10^{-6}$.

Alternatively, the variation of the modulus of $\boldsymbol{\omega}(r)$ in the interior of the flow at low Ekman numbers can be associated with the presence of a multitude of internal shear layers, which increase in number decreasing $E$.

Figures 2.3, 2.4, 2.5 show the velocity field in meridional planes, in a frame of reference 

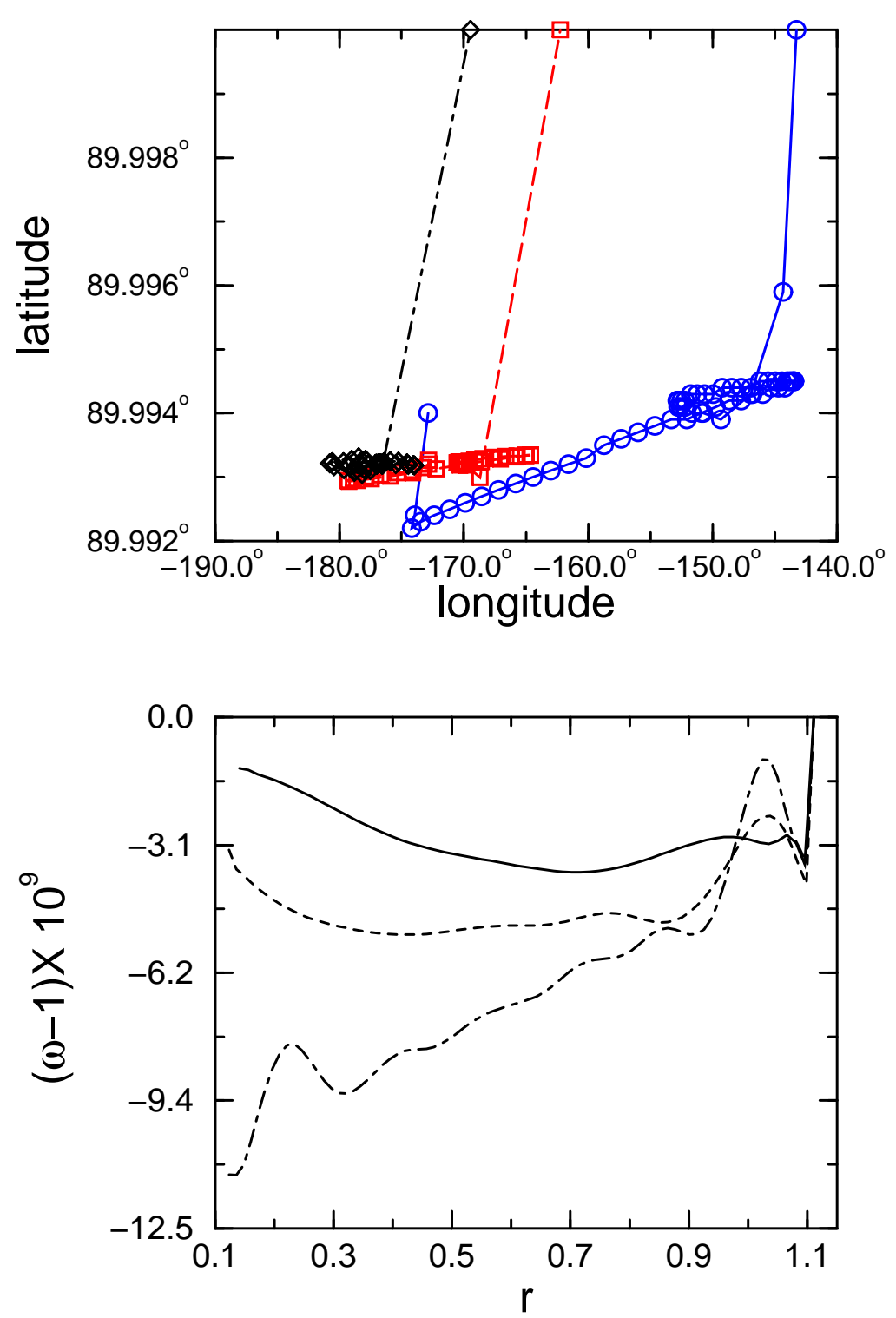

Figure 2.2: Top: Latitude and longitude of the direction of the fluid rotation vector $\boldsymbol{\omega}(r)$ (equ.(2.47)) as a function of $r$ for $\Omega=-10^{-5}, \alpha=30^{\circ}, \eta=0.1, e=0.04$ in the cases $E=10^{-4}$ (circles), $10^{-5}$ (squares) and $10^{-6}$ (diamonds). The symbols mark the direction of $\boldsymbol{\omega}(r)$ at the radii $r_{i}+\left(r_{o}-r_{i}\right) / 41 \cdot n, n=1 . .41$. The axis of (retrograde) precession points at zero longitude and a latitude of $60^{\circ}$. Bottom: $\omega$, the absolute value of $\boldsymbol{\omega}(r)$ (equ.(2.47)) as a function of $r$ for the same parameters as in the top panel with the Ekman numbers $E=10^{-4}$ (solid), $10^{-5}$ (dashed) and $10^{-6}$ (dot dashed). 
in which the rotation axis of the fluid $\boldsymbol{\omega}_{F}$ points in the $\hat{\boldsymbol{z}}$ direction.

Having in mind geophysical applications, the investigation has been extended to ellipsoidal shells with and without a sizeable inner core. Occasional comparisons with spherical geometries have been also included. The solid-body rotation has been subtracted out in all the figures to focus only on the viscous corrections.

The most apparent features are non-axisymmetric shear layers (inclined at $30^{\circ}$ to the fluid's axis) spawned by the critical latitudes which are separated by an angle of $60^{\circ}$ from the axis of rotation of the fluid (or alternatively by an angle of $30^{\circ}$ from the equator).

As the Ekman number decreases, the internal structures become much more localized and a comparison with the theory is easier.

Verifying the asymptotic scalings reported in section (1.3) is rather difficult, above all because it is unclear how to estimate the width of the shear layers. However, it is quite evident that the Ekman layer breaks down over a broad range in latitude and that inclined shear layers emanate from this entire range.

It is clear that the width of these layers is not inconsistent with an $O\left(E^{1 / 5}\right)$ scaling. This would imply, for instance, that going from $E=10^{-5}$ to $E=10^{-6}$ the layers become thinner by a factor of $10^{1 / 5}=1.6$, which is observed in figure 2.3 in the $\varphi^{\prime}$ component of the velocity, while the $r$ and $\vartheta^{\prime}$ components exhibit roughly a smaller factor (about 1.4).

Figures 2.4 and 2.5 show the effect of introducing an inner core.

Of the three possible directions along which the shear layers can be generated (see section 1.3), only the one tangential to the inner Ekman layer eruption and the one nontangential to the outer Ekman layer eruption are visible in the plots. The shear layer non-tangential to the inner eruption does not seem to be excited at all, as already pointed out by Hollerbach \& Kerwell (1995), Tilgner (1999).

Because the tangential shear layer is spawned by an $O\left(E^{2 / 5}\right)$, as opposed to an $O\left(E^{1 / 5}\right)$, generating region, it appears considerably thinner.

As shown in figure 2.5, in spherical geometry no qualitative, but only quantitative differences exist, among which is noteworthy the strengthening of the shear layer reflection pattern due to the tangential layer spawned by the critical latitude of the inner core.

The most striking features observed in experimental visualizations of the laminar flows (see figure 1.8) are cylindrical shear layers coaxial with the rotation axis of the fluid. Figure 2.6 therefore shows the flow component axisymmetric about $\boldsymbol{\omega}_{F}$.

The shear layers in the zonal velocity so evident in the experiments of Malkus (1968) and Vanyo et al. (1995), clearly appear in the plots. The exact location of the shear layers depends on both the boundary geometry and the Ekman number. A better comparison is possible in figure 2.7 in which the profiles of the zonal velocity in the plane perpendicular to $\boldsymbol{\omega}_{F}$ are plotted as a function of radius. The strongest shear layer connects the critical latitudes. A similar mean flow shear layer was derived analytically by Busse (1968) to explain the experimental observations of Malkus (1968). The asymptotic theory of Busse considered the Ekman layer breakdown at critical latitudes and the one mean flow shear layer spawned there. In the full problem addressed by the numerical simulations, also the inclined oscillatory shear layers exhibited from the basic flow have been taken into account which are responsible for the appearence of more than one mean flow shear layer. 

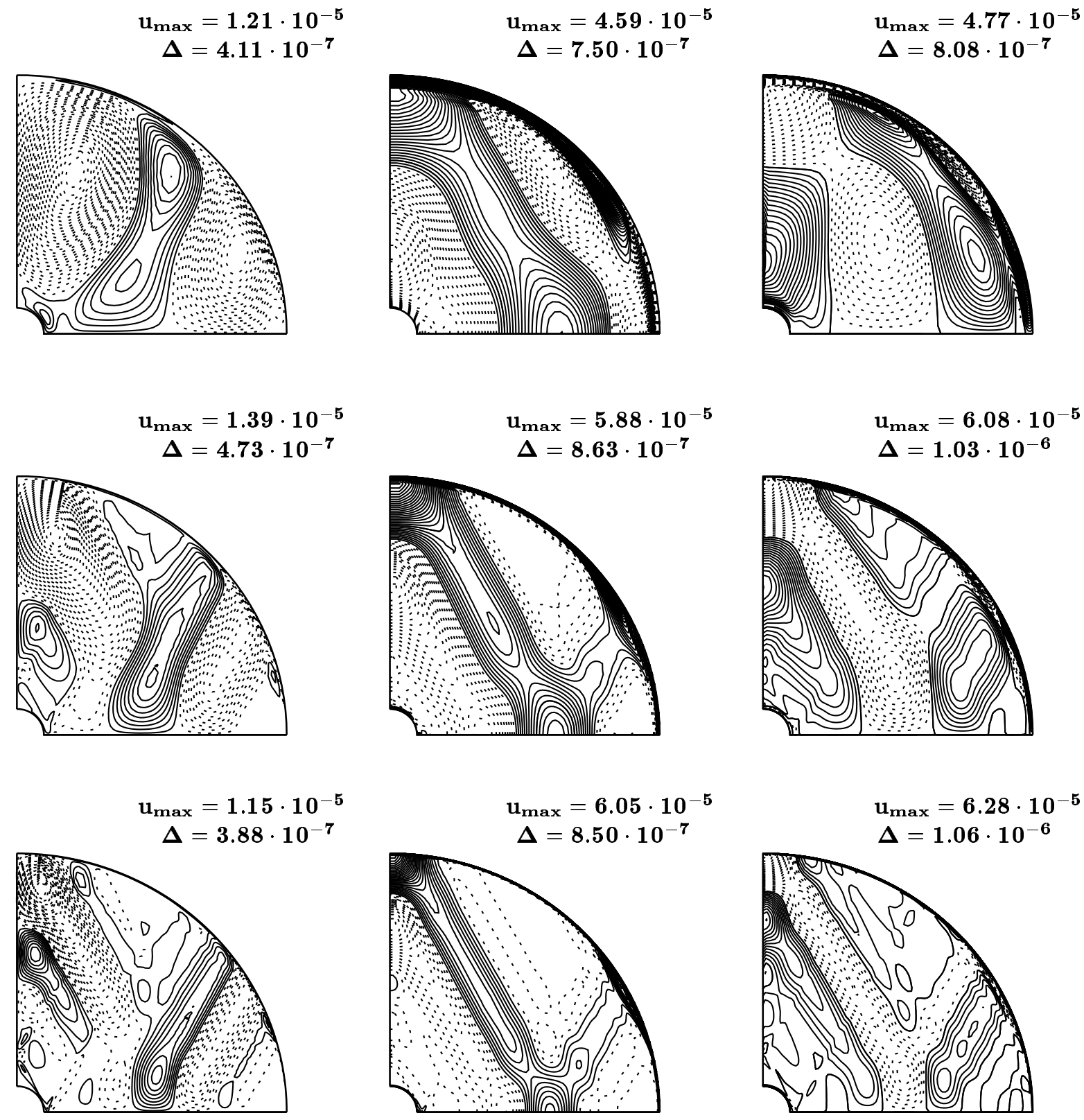

Figure 2.3: $r, \vartheta^{\prime}, \varphi^{\prime}$-components of $\boldsymbol{u}$ (from left to right) in meridional planes (where $\vartheta^{\prime}$ and $\varphi^{\prime}$ denote the angles with respect to the $\boldsymbol{\omega}_{F}$-axis), after subtraction of the average rotation of the fluid. Each component is shown in the plane in which it is strongest. $\eta=0.1, e=0.04, \Omega=-10^{-5}$ and $\alpha=30^{\circ}$ in all cases. From top to bottom, the Ekman number is $10^{-4}, 10^{-5}, 10^{-6}$. Solid and dashed contour lines indicate positive and negative values of the plotted quantity, respectively. Contour level separation $(\Delta)$ and values of the extreme levels $\left(u_{\max }\right)$ are given. 

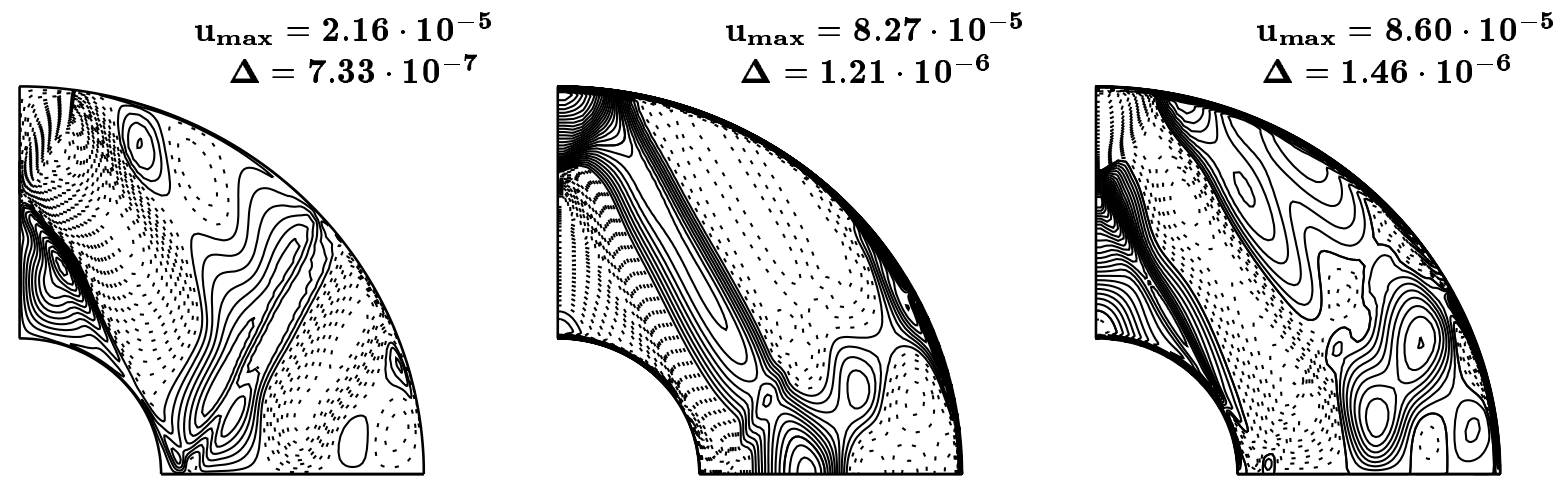

Figure 2.4: $r, \vartheta^{\prime}$ and $\varphi^{\prime}$-components of $\boldsymbol{u}$ (from left to right) in meridional planes (where $\vartheta^{\prime}$ and $\varphi^{\prime}$ denote the angles with respect to the $\boldsymbol{\omega}_{F}$-axis), after subtraction of the average rotation of the fluid. Each component is shown in the plane in which it is strongest. $\eta=0.35, e=0.04, E=10^{-5}, \alpha=30^{\circ}$ and $\Omega=-10^{-5}$.

The positions of the strongest prograde and retrograde jets correspond exactly to those given in figure 3 of Malkus (1968) and reported in section 1.4 (figure 1.7).

The position of the smaller extrema depends on $E$ and a perfect agreement with Malkus' figure cannot be expected for this part of the profile. As can be deduced from figure 2.7, the prograde jet becomes stronger with decreasing $E$ : a singularity should develop in the limit $E \rightarrow 0$, as already Busse outlined (1968).

The Ekman number dependence of the maximum zonal velocity does not follow any simple law valid for the entire interval $10^{-4}<E<10^{-6}$ but is compatible with a scaling in $E^{-3 / 10}$ for $10^{-5}<E<10^{-6}$ (Noir et al. 2001). Moreover, the number of these coaxial shear layers seems to increase with decreasing Ekman number.

The effect of introducing a sizeable inner core can be evaluated looking at figure 2.8, which shows the zonal flow symmetric about the fluid axis for both spherical and ellipsoidal shells.

The main cylindrical shear layer is hardly modified by the inner core, but a new one emerges near the critical latitudes of the inner core together with a tangential (inclined) shear layer. The conclusion is that for axisymmetric shear layers all the three characteristic directions are visible in the numerical simulations.

The meridional circulation (figure 2.9) shows that fluid flows from the poles to the equatorial plane along the rotation axis of the fluid.

This circulation is symmetric with respect to the equatorial plane. Vanyo \& Dunn (2000) have observed meridional flow traversing the equatorial plane even in purely laminar flow. This behaviour is not reproduced in our calculations.

In this section, fluid patterns generated by small precession rates have been taken into account, in order to obtain undoubtedly stable flows which could be compared with previous investigations. Also for the Earth, $\Omega \simeq-10^{-7}$. 

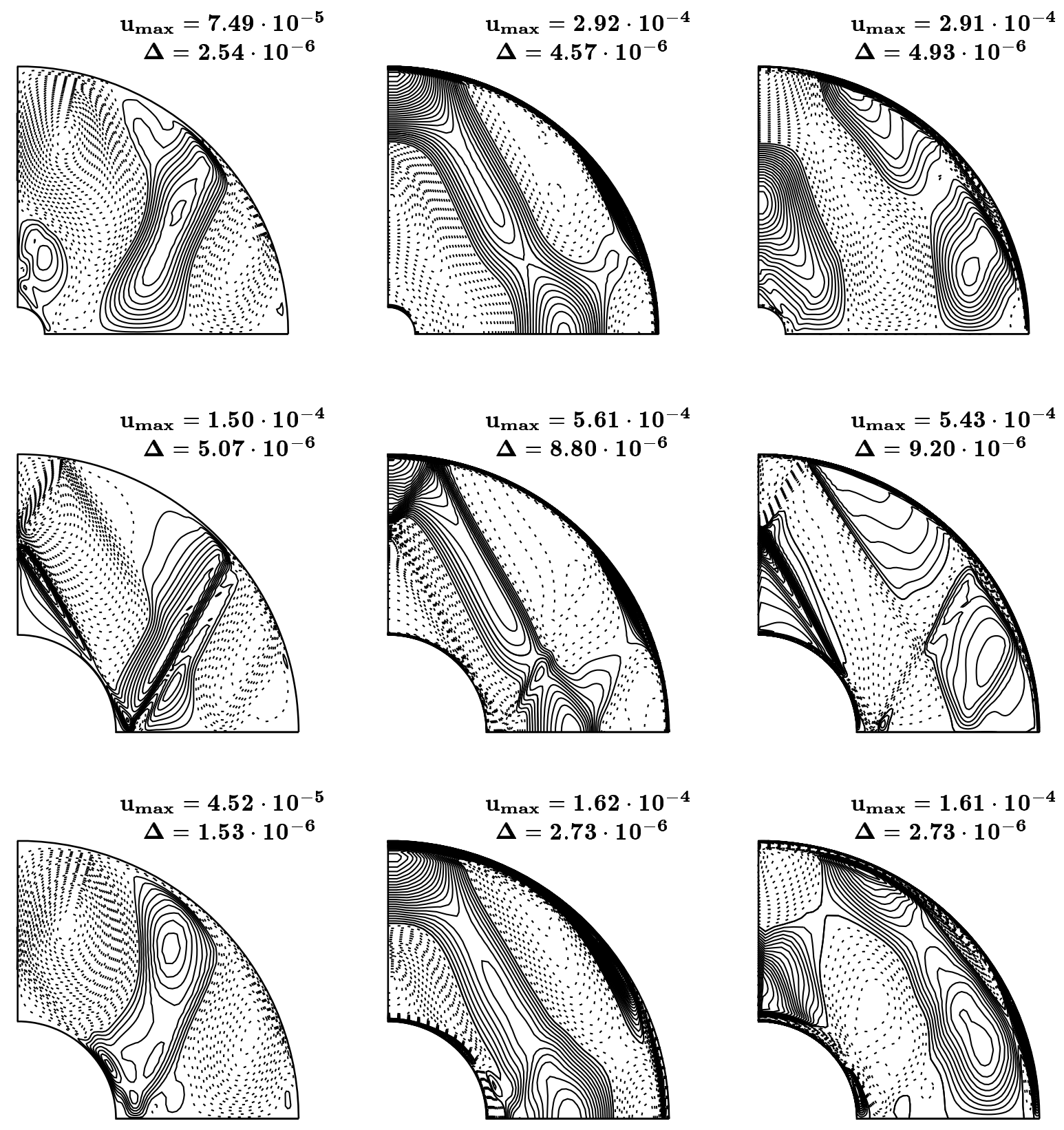

Figure 2.5: $r, \vartheta^{\prime}$ and $\varphi^{\prime}$-components of $\boldsymbol{u}$ (from left to right) in meridional planes (where $\vartheta^{\prime}$ and $\varphi^{\prime}$ denote the angles with respect to the $\boldsymbol{\omega}_{F}$-axis), after subtraction of the average rotation of the fluid. Each component is shown in the plane in which it is strongest. $e=0, \Omega=-10^{-5}$ and $\alpha=30^{\circ}$ in all cases. The remaining parameters are: (a) $E=10^{-5}$, $\eta=0.1$ (top panel); (b) $E=10^{-5}, \eta=0.35$ (middle panel); (c) $E=10^{-4}, \eta=0.35$ (bottom panel). 

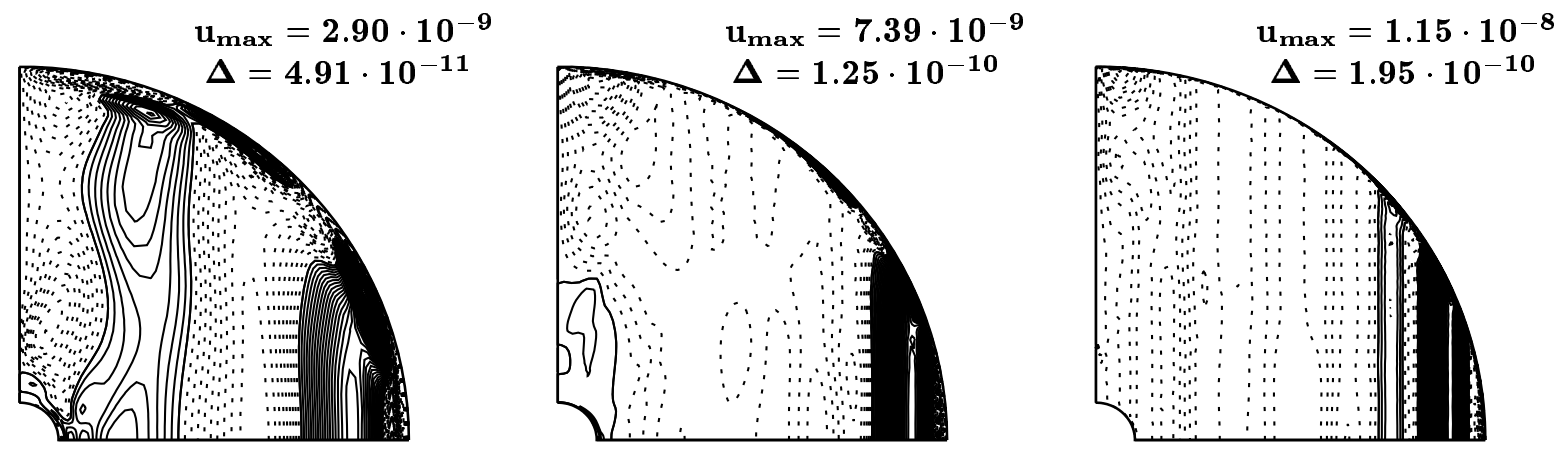

Figure 2.6: Zonal flow component $u_{\varphi^{\prime}}$ (where $\varphi^{\prime}$ denotes the azimuthal angle with respect to the $\boldsymbol{\omega}_{F}$-axis) axisymmetric about $\boldsymbol{\omega}_{F}$, after subtraction of the average rotation of the fluid. $\boldsymbol{\omega}_{F}$ is pointing upwards in all panels. $\eta=0.1, e=0.04, \Omega=-10^{-5}$ and $\alpha=30^{\circ}$ in all cases. From left to right, the Ekman number is $10^{-4}, 10^{-5}, 10^{-6}$.

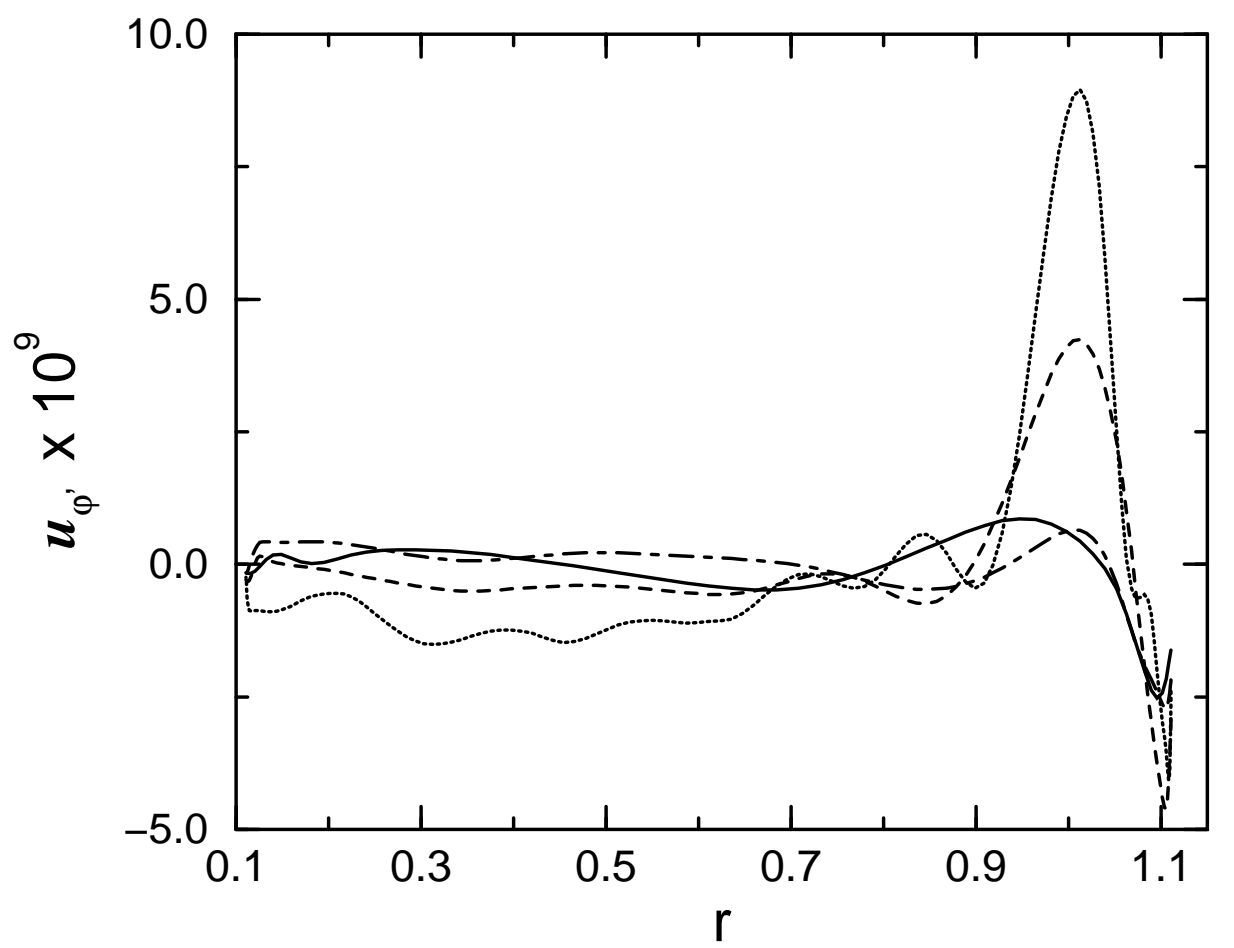

Figure 2.7: $u_{\varphi^{\prime}}$ from figure 2.6 in the equatorial plane as a function of $r$ for $\Omega=-10^{-5}$, $\alpha=30^{\circ}$ and $\eta=0.1$. The line styles indicate $e=0.04, E=10^{-4}$ (solid), $e=0.04$, $E=10^{-5}$ (dashed), $e=0.04, E=10^{-6}$ (dotted), and $e=0, E=10^{-5}$ (dot dashed). For this last case, $u_{\varphi^{\prime}}$ has been divided by 100 in order to make the curve fit into the figure. 

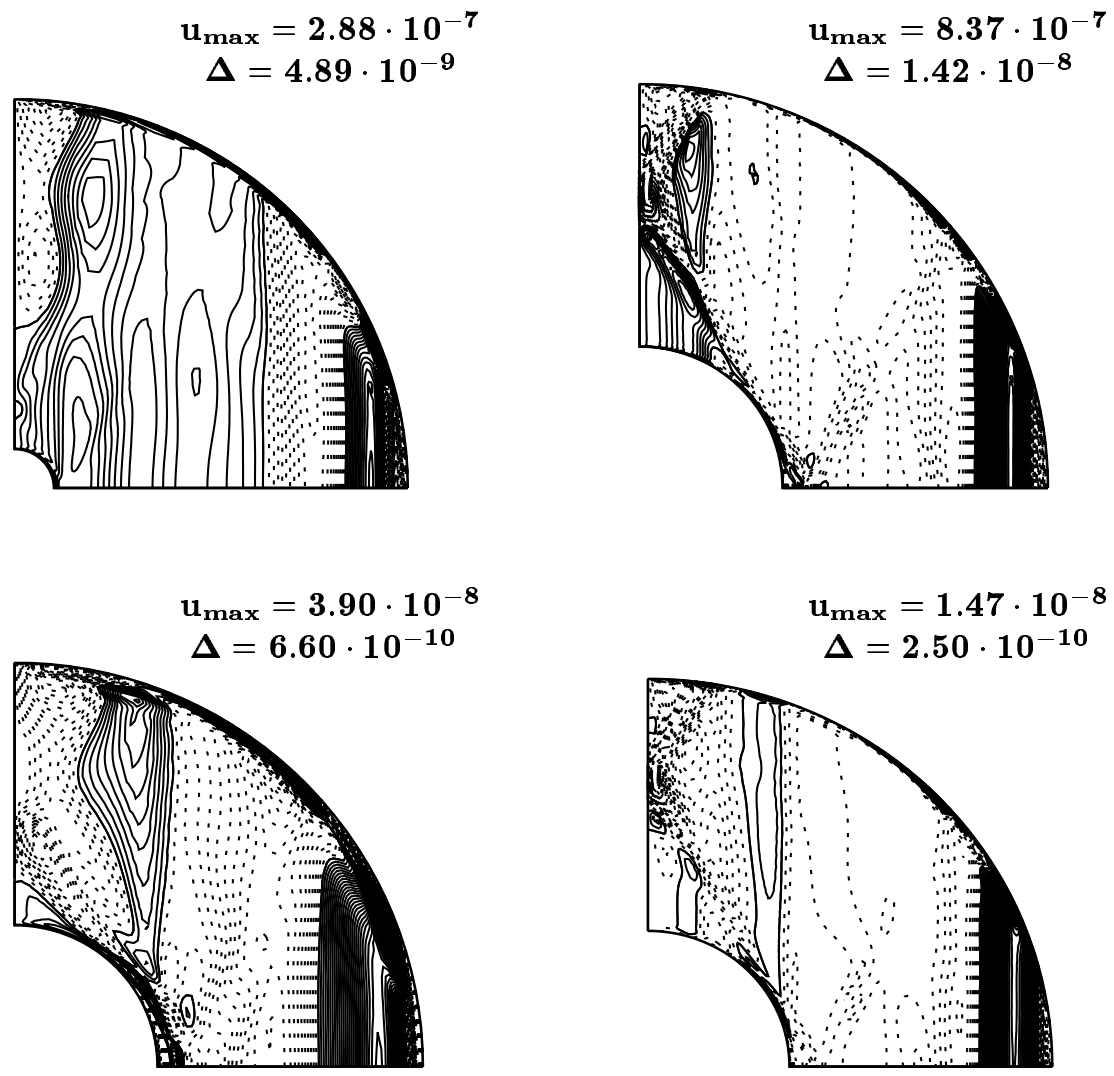

Figure 2.8: $u_{\varphi^{\prime}}$ as in figure 2.6 for $\Omega=-10^{-5}$ and $\alpha=30^{\circ}$. The remaining parameters are: (top-left) $E=10^{-5}, e=0, \eta=0.1$, (top-right) $E=10^{-5}, e=0, \eta=0.35$, (bottom-left) $E=10^{-4}, e=0, \eta=0.35$, (bottom-right) $E=10^{-5}, e=0.04, \eta=0.35$. 

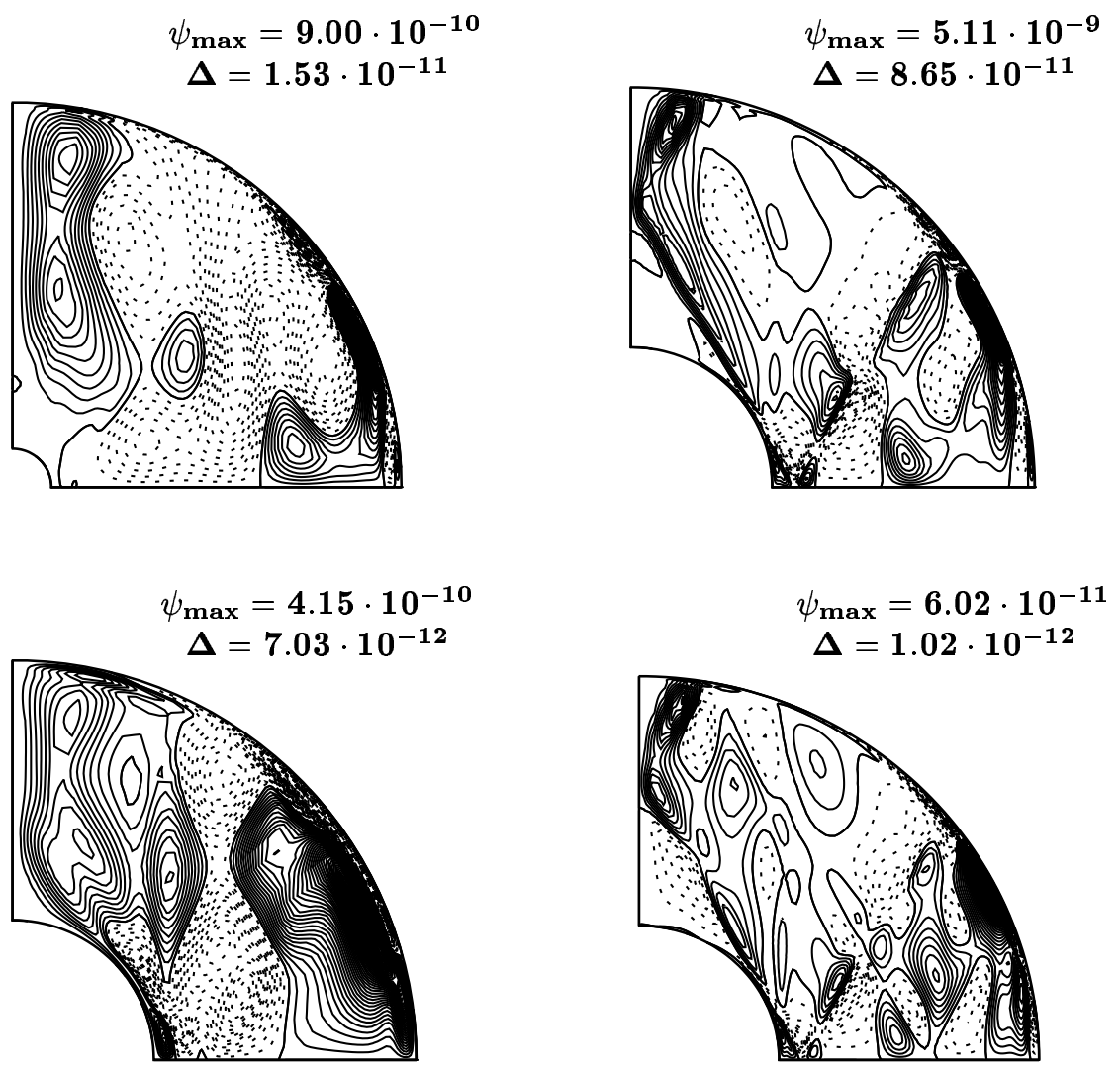

Figure 2.9: Streamlines of the meridional component axisymmetric about $\boldsymbol{\omega}_{F}$, for the same parameters as in figure 2.8, i.e. $\Omega=-10^{-5}$ and $\alpha=30^{\circ}$. The remaining parameters are: (top-left) $E=10^{-5}, e=0, \eta=0.1$, (top-right) $E=10^{-5}, e=0, \eta=0.35$, (bottomleft) $E=10^{-4}, e=0, \eta=0.35$, (bottom-right) $E=10^{-5}, e=0.04, \eta=0.35$. Contour lines are drawn for levels ranging from $-\psi_{\max }$ to $+\psi_{\max }$. The values of adjacent levels differ by $\Delta$. 


\subsection{Instabilities}

The laminar flows described in the previous section are symmetric with respect to the reflection, through the origin, of a point $(r, \vartheta, \varphi)$ relative to a fixed Cartesian coordinate system.

This means, in terms of velocity field, that:

$$
\boldsymbol{u}(\boldsymbol{r})=-\boldsymbol{u}(-\boldsymbol{r})
$$

The characteristic parity shown by the basic flow follows immediately from the equation of motion in that its homogeneous part and the boundary conditions are invariant under reflection at the origin while the inhomogeneous part (Poincaré force) is symmetric.

The instabilities detected and presented in the following break this symmetry. It is therefore useful to separate the full velocity field into symmetric and antisymmetric components, such that:

$$
\boldsymbol{u}=\boldsymbol{u}_{s}+\boldsymbol{u}_{a}
$$

with: $\boldsymbol{u}_{s}=(\boldsymbol{u}(\boldsymbol{r})-\boldsymbol{u}(-\boldsymbol{r})) / 2$ and $\boldsymbol{u}_{a}=(\boldsymbol{u}(\boldsymbol{r})+\boldsymbol{u}(-\boldsymbol{r})) / 2$.

A convenient indicator for the onset of instability is the energy $E_{a}$ contained in the antisymmetric velocity field $\boldsymbol{u}_{a}$ (i.e. poloidal coefficients $V_{l}^{m}$ with odd $l$ and toroidal coefficients $W_{l}^{m}$ with even $l$, since the parity of the spherical harmonics $Y_{l}^{m}(\vartheta, \varphi)$ is $\left.(-1)^{l}\right)$.

Table 2.2 lists $E_{a}$ for flows above the threshold of the instability, while table 2.3 gives examples of stable flows.

The parameters used for the simulations have been selected in order to reasonably reproduce the experiments reported in section 1.4, in particular the original experiments performed by Malkus (1968), which are closest to the computational accessible regime.

By looking at table 2.2 is evident that, increasing the Ekman number, the instability sets in at higher precession rates.

Accordingly, an exact correspondence with the experiments cannot be expected as concerns the threshold precession rates at which the instability appears.

The experiments carried out by Vanyo et al. (1995) (whose findings are summarized in figure 1.8, section 1.4) suggest that one should look for disturbances with a well defined wavenumber with respect to the fluid axis.

The velocity fields have therefore been transformed to a coordinate system denoted by primes in which the $z^{\prime}$-axis points along $\boldsymbol{\omega}_{F}$. The field has then been decomposed into spherical harmonics in this new system and the energy contained in the different spectral components has been plotted against the azimuthal wavenumber $m^{\prime}$.

Figure 2.10 shows a particular example, in which a dominant contribution to $\boldsymbol{u}_{a}$ occurs at $m^{\prime}=7$ and 8 , whereas $\boldsymbol{u}_{s}$ differs little from its shape in stable solutions and certainly does not contain any hint at a wave propagating around the $z^{\prime}$-axis. 


$\begin{array}{ccccccccccc}E & e & \alpha & \Omega & \eta & E_{k i n} & \tilde{E}_{k i n} & E_{a} & R e_{b l} & R o_{b l} & \\ & & & & & & & & & & \\ 5 \times 10^{-4} & 0.1 & 80^{\circ} & -0.08 & 0.1 & 0.920 & 0.115 & 5.92 \times 10^{-4} & 58 & 0.40 & \mathrm{v} \\ 5 \times 10^{-4} & 0.1 & 80^{\circ} & -0.2 & 0.1 & 1.22 & 0.20 & 2.19 \times 10^{-3} & 56 & 0.46 & \mathrm{v} \\ 10^{-4} & 0.1 & 80^{\circ} & -0.08 & 0.1 & 1.33 & 0.11 & 1.10 \times 10^{-2} & 140 & 0.46 & \mathrm{bl} \\ & & & & & & & & & & \\ 10^{-4} & 0.04 & 30^{\circ} & -0.03 & 0.1 & 0.373 & 2.25 \times 10^{-2} & 4.25 \times 10^{-4} & 91 & 0.25 & \mathrm{v}, \mathrm{bl} \\ 10^{-4} & 0.04 & 30^{\circ} & -0.035 & 0.1 & 0.49 & 3.1 \times 10^{-2} & 8.1 \times 10^{-4} & 104 & 0.28 & \mathrm{v}, \mathrm{bl} \\ 10^{-4} & 0.04 & 30^{\circ} & -0.05 & 0.1 & 0.573 & 3.70 \times 10^{-2} & 1.56 \times 10^{-3} & 113 & 0.31 & \mathrm{v}, \mathrm{bl} \\ 10^{-4} & 0.04 & 30^{\circ} & -0.1 & 0.1 & 0.512 & 3.18 \times 10^{-2} & 6.25 \times 10^{-4} & 107 & 0.31 & \mathrm{v}, \mathrm{bl} \\ 10^{-4} & 0.04 & 30^{\circ} & -0.2 & 0.1 & 0.435 & 3.27 \times 10^{-2} & 9.38 \times 10^{-4} & 94 & 0.30 & \mathrm{v}, \mathrm{bl} \\ 2 \times 10^{-5} & 0.04 & 30^{\circ} & -0.018 & 0.1 & 0.161 & 4.44 \times 10^{-3} & 5.00 \times 10^{-6} & 109 & 0.16 & \mathrm{v}, \mathrm{bl} \\ & & & & & & & & & & \\ 7 \times 10^{-4} & 0 & 60^{\circ} & -0.2 & 0.01 & 0.609 & 0.12 & 7.67 \times 10^{-5} & 54 & 0.34 & \mathrm{v} \\ 5 \times 10^{-4} & 0 & 60^{\circ} & -0.1 & 0.01 & 0.509 & 6.79 \times 10^{-2} & 5.21 \times 10^{-4} & 45 & 0.32 & \mathrm{v} \\ 10^{-4} & 0 & 40^{\circ} & -0.05 & 0.1 & 0.500 & 3.14 \times 10^{-2} & 4.25 \times 10^{-4} & 104 & 0.28 & \mathrm{v}, \mathrm{bl}\end{array}$

Table 2.2: Parameters for the simulations in ellipsoids and a few runs in spherical geometry near the onset of instability. $E_{k i n}$ is the total energy in the mantle frame, $\tilde{E}_{k i n}$ the energy in components other than $W_{1}^{0}$ and $W_{1}^{1}$ (equation (2.47)), and $E_{a}$ the energy of $\boldsymbol{u}_{a} . \quad R e_{b l}$ and $R o_{b l}$ are the Reynolds and Rossby numbers of the boundary layer. The last column states whether the instability occurred in the boundary layer (bl) or in the volume (v). Resolutions ranged from 65 to 129 Chebychev polynomials and from 64 to 128 for the highest order of retained spherical harmonics. The highest included azimuthal wavenumbers ranged from 32 to 64 . 


$\begin{array}{ccccccc}E & e & \alpha & \Omega & \eta & E_{k i n} & \tilde{E}_{k i n} \\ & & & & & & \\ 5 \times 10^{-4} & 0.1 & 80^{\circ} & -0.03 & 0.1 & 7.63 \times 10^{-2} & 7.94 \times 10^{-3} \\ 10^{-4} & 0.1 & 80^{\circ} & -0.01 & 0.1 & 1.03 \times 10^{-2} & 5.42 \times 10^{-4} \\ & & & & & & \\ 10^{-4} & 0.04 & 30^{\circ} & -0.01 & 0.1 & 1.84 \times 10^{-2} & 1.01 \times 10^{-3} \\ 3 \times 10^{-5} & 0.04 & 30^{\circ} & -0.01 & 0.1 & 2.65 \times 10^{-2} & 8.90 \times 10^{-4} \\ 2 \times 10^{-5} & 0.04 & 30^{\circ} & -0.01 & 0.1 & 2.85 \times 10^{-2} & 7.98 \times 10^{-4} \\ 10^{-5} & 0.04 & 30^{\circ} & -0.001 & 0.1 & 1.96 \times 10^{-4} & 3.68 \times 10^{-6} \\ & & & & & & \\ 5 \times 10^{-4} & 0 & 60^{\circ} & -0.07 & 0.01 & 3.94 \times 10^{-1} & 4.78 \times 10^{-2} \\ 10^{-4} & 0 & 30^{\circ} & -0.01 & 0.1 & 5.48 \times 10^{-2} & 3.31 \times 10^{-3}\end{array}$

Table 2.3: Same as table 2.2 for runs below the onset of instability.

In order to describe the wavelike character of the instability, it is thus enough to consider the structure of $\boldsymbol{u}_{a}$. Figures 2.11, 2.12 and 2.13 clarify the structure of the instability for the same set of parameters used in figure 2.10. Figures 2.11 and 2.12 are snapshots. As time goes on, the $m^{\prime}=7$ and $m^{\prime}=8$ patterns rotate independently of each other about the $z^{\prime}$-axis. Figure 2.11 shows $u_{a r}$ and $u_{a z^{\prime}}$ in the plane perpendicular to $\boldsymbol{\omega}_{F}$ (equatorial plane). $m^{\prime}=7$ appears in $u_{a r}$ whereas $m^{\prime}=8$ dominates $u_{a z^{\prime}}$.

The two sets of rolls overlap but are centered at different radii. Figure 2.12 shows cylindrical cuts at distances from the fluid axis corresponding to these two radii. The columnar vortices corresponding to $m^{\prime}=7$ are symmetric about the equatorial plane of the primed coordinate system, whereas the $m^{\prime}=8$ vortices are antisymmetric about this plane. Figure 2.13 displays the most striking features of the instability in a sketch. Two sets of columnar vortices centered at different distances from the fluid axis exist, with wavenumbers differing by one. The entire pattern is antisymmetric with respect to reflection at the origin. Individual rolls in the wave with odd wavenumber therefore have equal vorticity in the northern and southern hemisphere. Rolls belonging to the wave with even wavenumber, on the contrary, have opposite vorticities in both hemispheres. The axial and azimuthal components in the outer roll pattern are of comparable magnitude, whereas $u_{a z^{\prime}}$ is small in the component with odd $m^{\prime} . u_{a z^{\prime}}$ reaches its extremal values in between the outer vortices.

The characteristics of the instability so far described have a general relevance, in the sense that the same sketch is valid for other parameters, except that the values of $m^{\prime}$ change as indicated in table 2.4. 


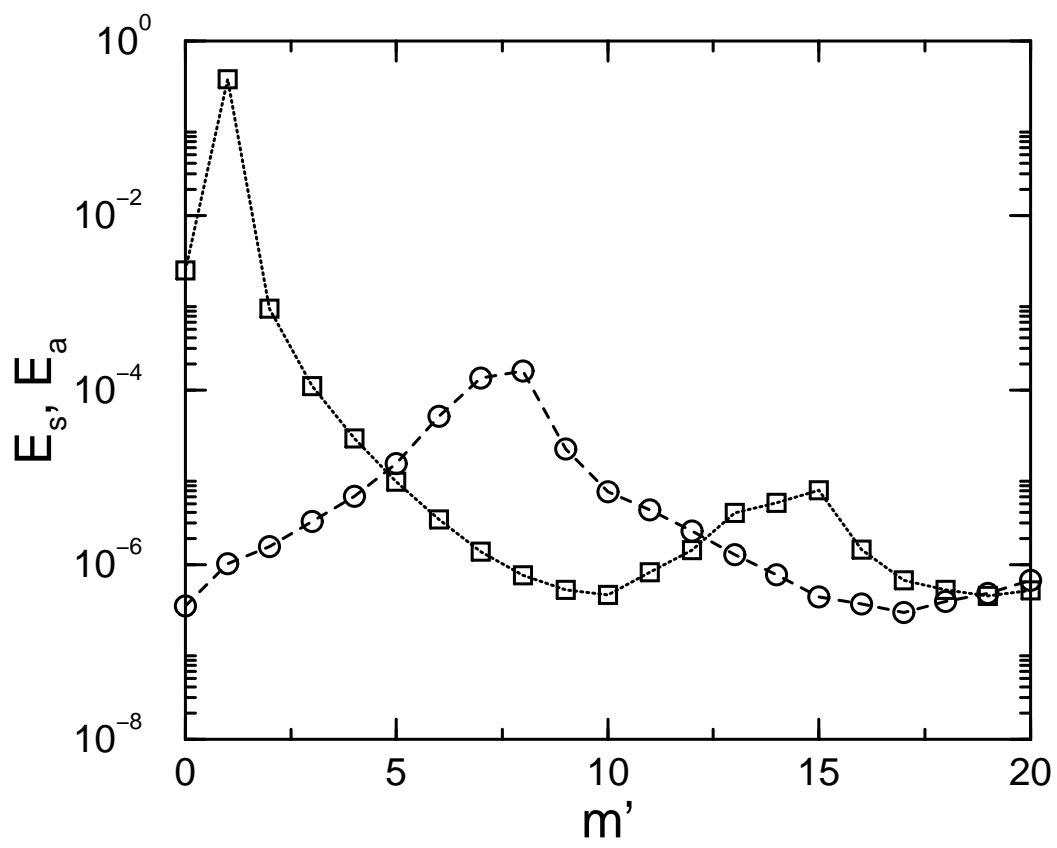

Figure 2.10: Energy contained in the modes with wavenumber $m^{\prime}$ as a function of $m^{\prime}$ for $\eta=0.1, e=0.04, \alpha=30^{\circ}, \Omega=-0.03$ and $E=10^{-4}$. Antisymmetric (circles) and symmetric (squares) contributions are shown separately.
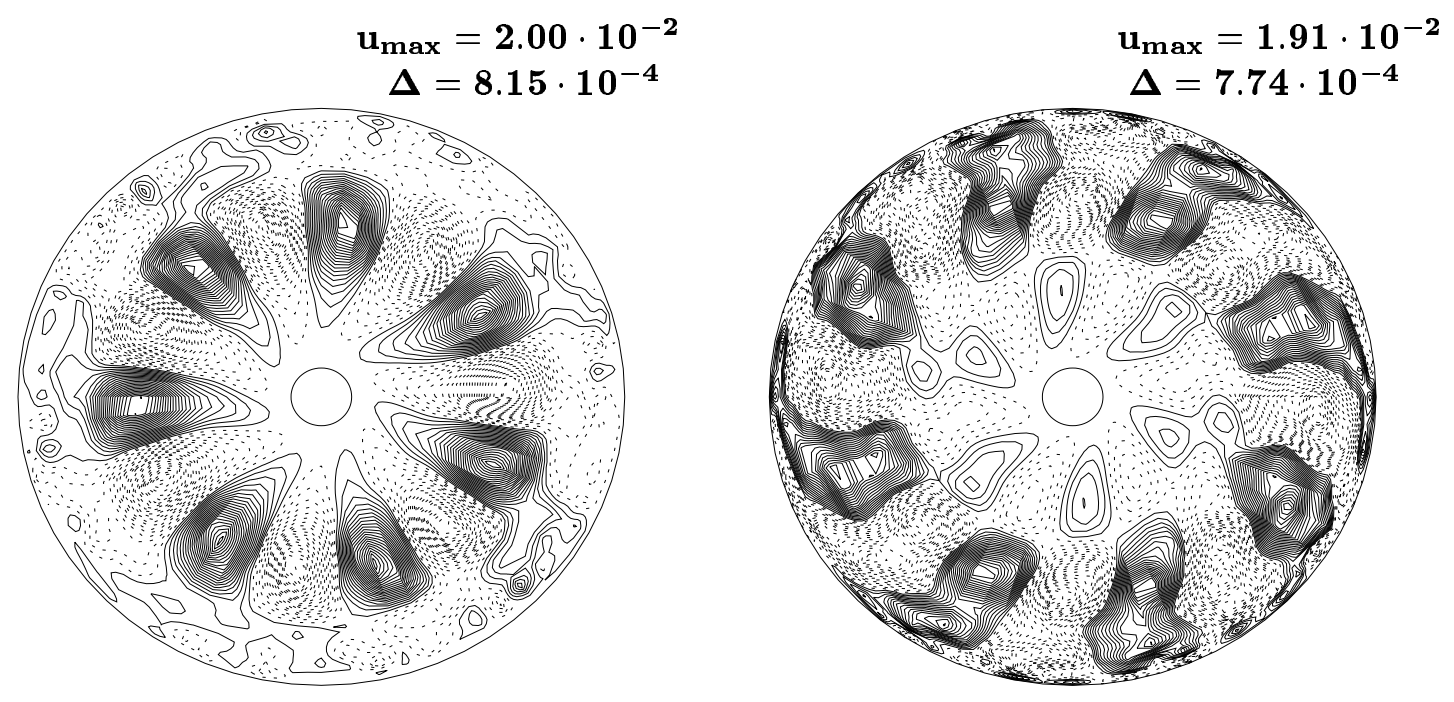

Figure 2.11: $u_{a r}$ (left) and $u_{a z^{\prime}}$ (right) in the plane perpendicular to $\boldsymbol{\omega}_{F}$ for the same parameters as in figure 2.10. Contour level separation $(\Delta)$ and values of the extreme levels $\left(u_{\max }\right)$ are given for both panels. 

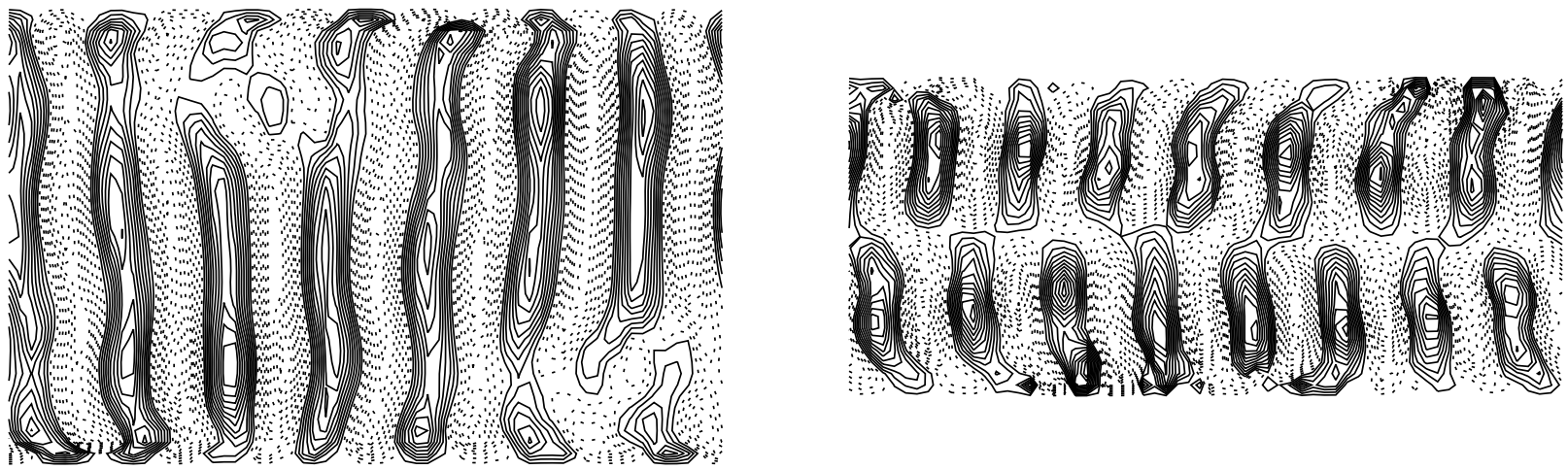

Figure 2.12: $u_{a r}$ on cylindrical surfaces at distances 0.638 (left) and 0.869 (right) from the $\boldsymbol{\omega}_{F}$-axis for the same case as in figures 2.10 and 2.11. $\varphi^{\prime}$ runs from $-\pi$ to $\pi$ in going from left to right and $\vartheta^{\prime}$ runs from -60 to 60 (left) and from -40 to 40 (right) in going from bottom to top.

$\begin{array}{ccccccccc}E & e & \alpha & \Omega & \eta & m^{\prime} & \omega_{m^{\prime}} & T & \left|\boldsymbol{\omega}_{F}\right| \\ & & & & & & & & \\ 10^{-4} & 0.04 & 30^{\circ} & -0.03 & 0.1 & 7,8 & \omega_{7}=6.28, \omega_{8}=6.23 & 0.50 & 0.888 \\ 5 \times 10^{-4} & 0.1 & 80^{\circ} & -0.08 & 0.1 & 3,4 & \omega_{3}=1.90, \omega_{4}=1.76 & 1.71 & 0.614 \\ 5 \times 10^{-4} & 0 & 60^{\circ} & -0.1 & 0.01 & 2,3,4 & \omega_{2}=2.15, \omega_{3}=2.12, \omega_{4}=2.12 & 1.48 & 0.681\end{array}$

Table 2.4: Details of the modes excited near the onset of instability. $m^{\prime}$ and $\omega_{m^{\prime}}$ are the wavenumber and drift frequency of these modes with respect to the rotation axis of the fluid. In a frame in which the precession and container axes are fixed and the fluid axis points along $z^{\prime}$, the temporal and azimuthal dependence of the waves is given by $e^{i\left(m^{\prime} \varphi^{\prime}-\omega_{m^{\prime}} t\right)}$. In this frame, the fluid rotates at angular velocity $\left|\boldsymbol{\omega}_{F}\right|$. The $\omega_{m^{\prime}}$ have been determined from equatorial sections as in figure 2.11 plotted for different moments in time. The energy of the flow oscillates with the period $T$. 


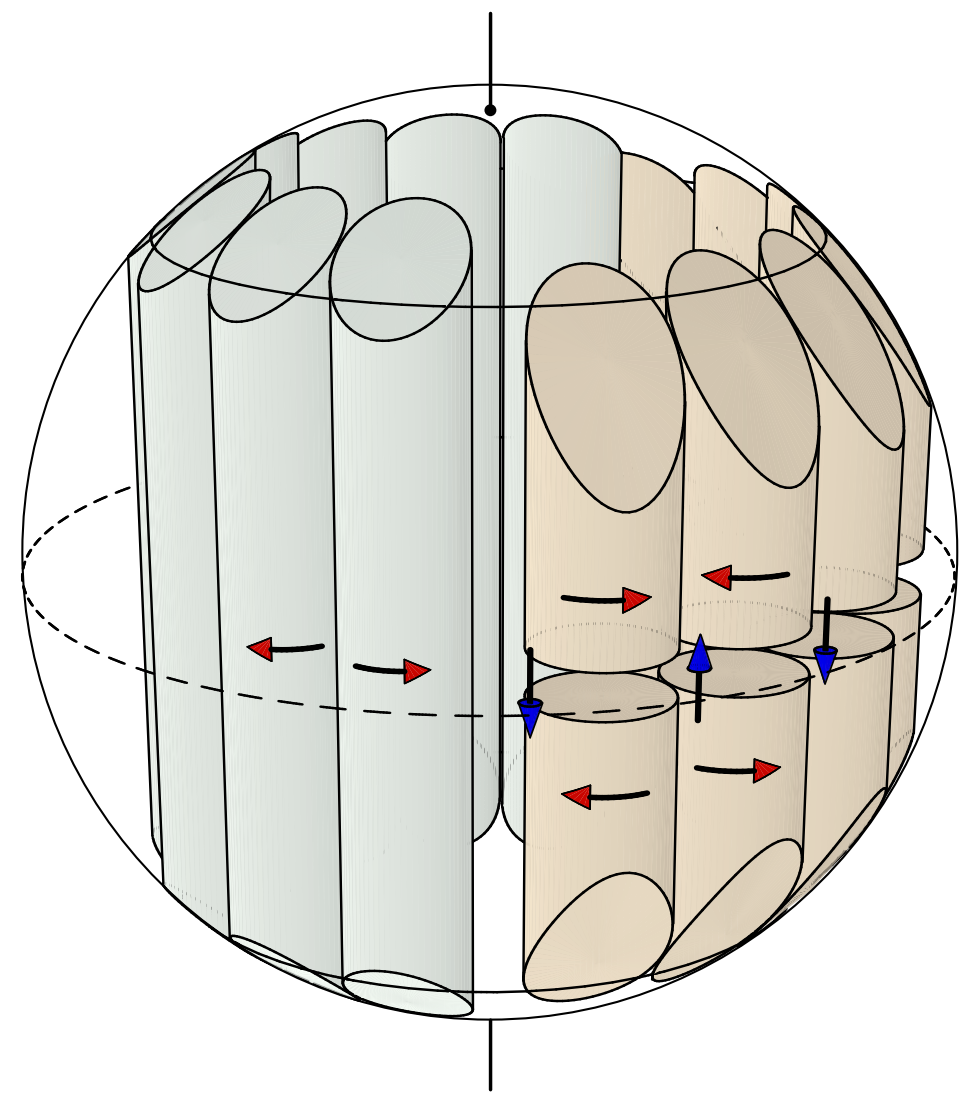

Figure 2.13: Sketch of the structure of the unstable mode in figures 2.11 and 2.12. 
In one case, corresponding to the spherical geometry, three adjacent values of $m^{\prime}$ are listed. Figure 2.14 shows that also in this case only two sets of rolls arise, because the superposition of the waves with the highest and lowest $m^{\prime}$ (which have the same symmetry with respect to the equatorial plane) merely appears as a time dependent distortion of the wave with the highest $m^{\prime}$. All the three modes drift independently of each other.
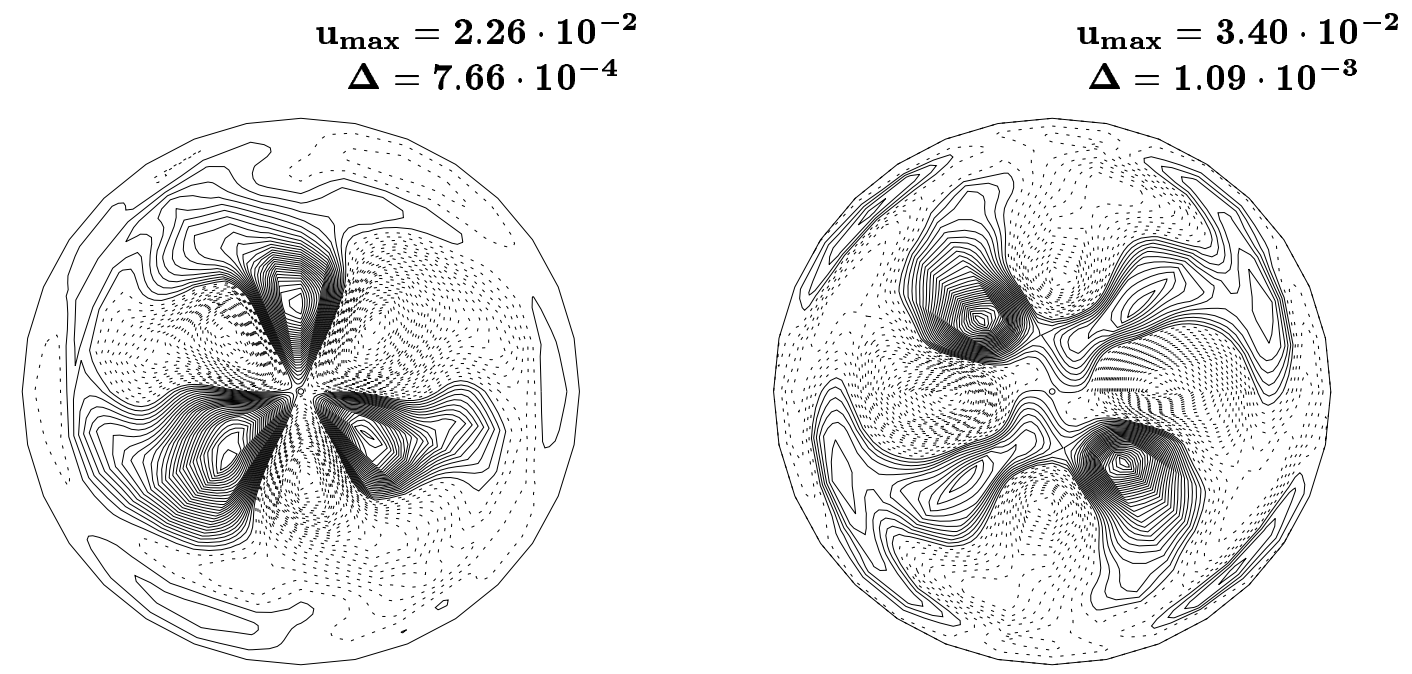

Figure 2.14: $u_{a r}$ (left) and $u_{a z^{\prime}}$ (right) in the plane perpendicular to $\omega_{F}$ for $\eta=0.01$, $e=0, \alpha=60^{\circ}, \Omega=-0.1$ and $E=5 \times 10^{-4}$.

In order to investigate some properties characterizing the detected instabilities, it is useful to introduce the separate equations of motion for the symmetric and antisymmetric components of the velocity field. In the precessing primed coordinate system, in which the fluid axis is along $z^{\prime}$ and the precession axis $\hat{\boldsymbol{\Omega}}$ and container axis $\hat{\boldsymbol{z}}$ are stationary, these equations read:

$$
\begin{aligned}
& \frac{\partial}{\partial t} \nabla \times \boldsymbol{u}_{s}+\nabla \times\left[\left(\nabla \times \boldsymbol{u}_{s}\right) \times \boldsymbol{u}_{s}+\left(\nabla \times \boldsymbol{u}_{a}\right) \times \boldsymbol{u}_{a}\right]+2 \nabla \times\left(\boldsymbol{\Omega} \times \boldsymbol{u}_{s}\right)=E \nabla^{2} \nabla \times \boldsymbol{u}_{s} \\
& \frac{\partial}{\partial t} \nabla \times \boldsymbol{u}_{a}+\nabla \times\left[\left(\nabla \times \boldsymbol{u}_{s}\right) \times \boldsymbol{u}_{a}+\left(\nabla \times \boldsymbol{u}_{a}\right) \times \boldsymbol{u}_{s}\right]+2 \nabla \times\left(\boldsymbol{\Omega} \times \boldsymbol{u}_{a}\right)=E \nabla^{2} \nabla \times \boldsymbol{u}_{a}
\end{aligned}
$$

If $\boldsymbol{r}$ is a vector pointing on the boundary, $\boldsymbol{u}_{s}=\hat{\boldsymbol{z}} \times \boldsymbol{r}$ and $\boldsymbol{u}_{a}=0$ on the boundary. The boundary conditions can only drive $\boldsymbol{u}_{s}$. If $\boldsymbol{u}_{a}$ is strictly zero initially, it will stay zero forever. Therefore, $\boldsymbol{u}_{a}$ can only grow as an instability of a ground state $\boldsymbol{u}_{s}$. Once a pair of modes has been excited in $\boldsymbol{u}_{a}$, it drives through the $\left(\nabla \times \boldsymbol{u}_{a}\right) \times \boldsymbol{u}_{a}$ term in (2.48) a symmetric motion at the double wavenumber. This can be seen looking at figure 2.10. 
The instability at onset manifests itself in small amplitude oscillations of the energy as in a Hopf bifurcation.

Even if the oscillation is not always exactly harmonic, an underlying period $T$ can be discerned, as reported in table 2.4 .

The temporal variations of the antisymmetric energy in the various states are shown in figure 2.15 for a representative case.

Close to the onset, the instability is oscillatory.

It has been verified that, once the antisymmetric energy has reached the final state, the total kinetic energy is driven at the same oscillatory regime with the same period $T$.

The sum of the two angular frequencies of a pair of modes is nearly $2 \pi / T$.

Increasing the precession rate far from the onset of the instability, a chaotic time dependence appears.

It is tempting to classify as Rossby waves the vortices which are symmetric with respect to the equatorial plane.

In order to do that, the frequency of the set of rolls corresponding to the odd wavenumbers $m^{\prime}$ has been calculated for a few representative cases reported in table 2.4.

In the frame of the fluid, these frequencies have been computed as:

$$
\omega_{R}=\omega_{m^{\prime}}-m^{\prime}\left|\boldsymbol{\omega}_{F}\right|
$$

For the examples in table 2.4 , with $\omega_{m^{\prime}}$ equal to $6.28,1.90,2.12$, one finds values for $\omega_{R}$ of $0.064,0.058$ and 0.077 , respectively.

A comparison has then been made with the frequencies obtained using equation (1.32) (section 1.1), where as distance $s_{o}$, at which the Rossby waves are localized, has been assumed the radius from the rotation axis of the fluid at which the set of rolls is centered.

The values obtained for $\omega_{R}$ are within 0.05 of the frequency computed by means of equation (1.32). A better agreement cannot be expected because of the uncertainties in the determination of $\omega_{m^{\prime}}$ and because the observed motion is not well localized at a certain cylindrical radius as is assumed in the theoretical formula.

All the three values found for $\omega_{R}$ corresponding to the symmetric vortices are positive as must be for Rossby waves. The same calculation for the antisymmetric vortices yields mostly negative $\omega_{R}$.

Figure 2.16 shows two series of simulations in which $|\boldsymbol{\Omega}|$ has been systematically increased, in ellipsoidal and spherical geometry.

The graphic reveals that the antisymmetric energy is not a growing function of the precession rate, but surprisingly it can drop back to zero for $|\boldsymbol{\Omega}|$ above the instability threshold. One could suspect that an instability shows in the symmetric components at these parameters, but this is not the case. 

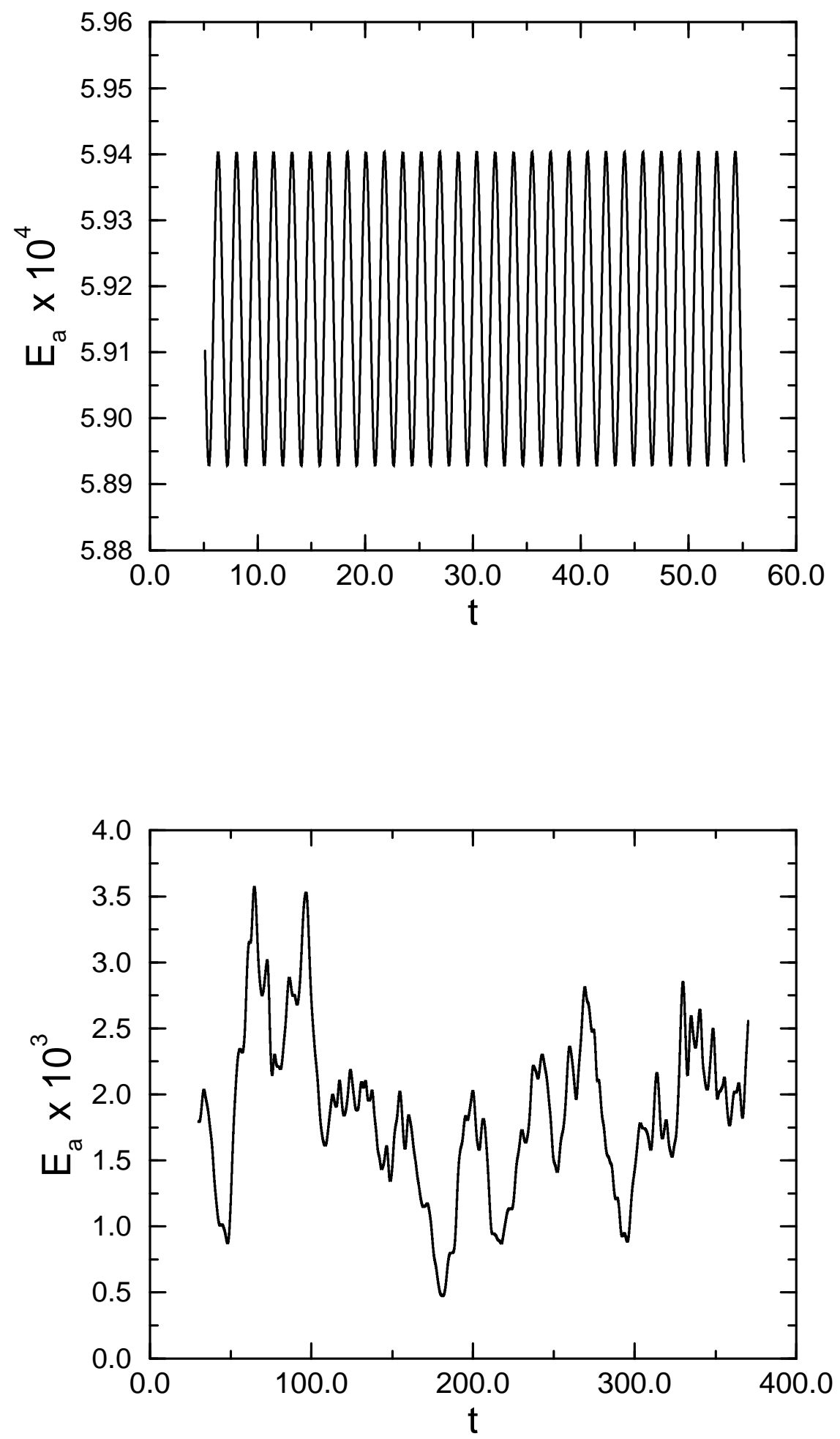

Figure 2.15: Time dependence of the antisymmetric energy in a shell with $\eta=0.1, e=0.1$, $\alpha=80^{\circ}, E=5 \times 10^{-4}$. $\Omega$ is -0.08 in the top panel and -0.2 in the bottom panel. 


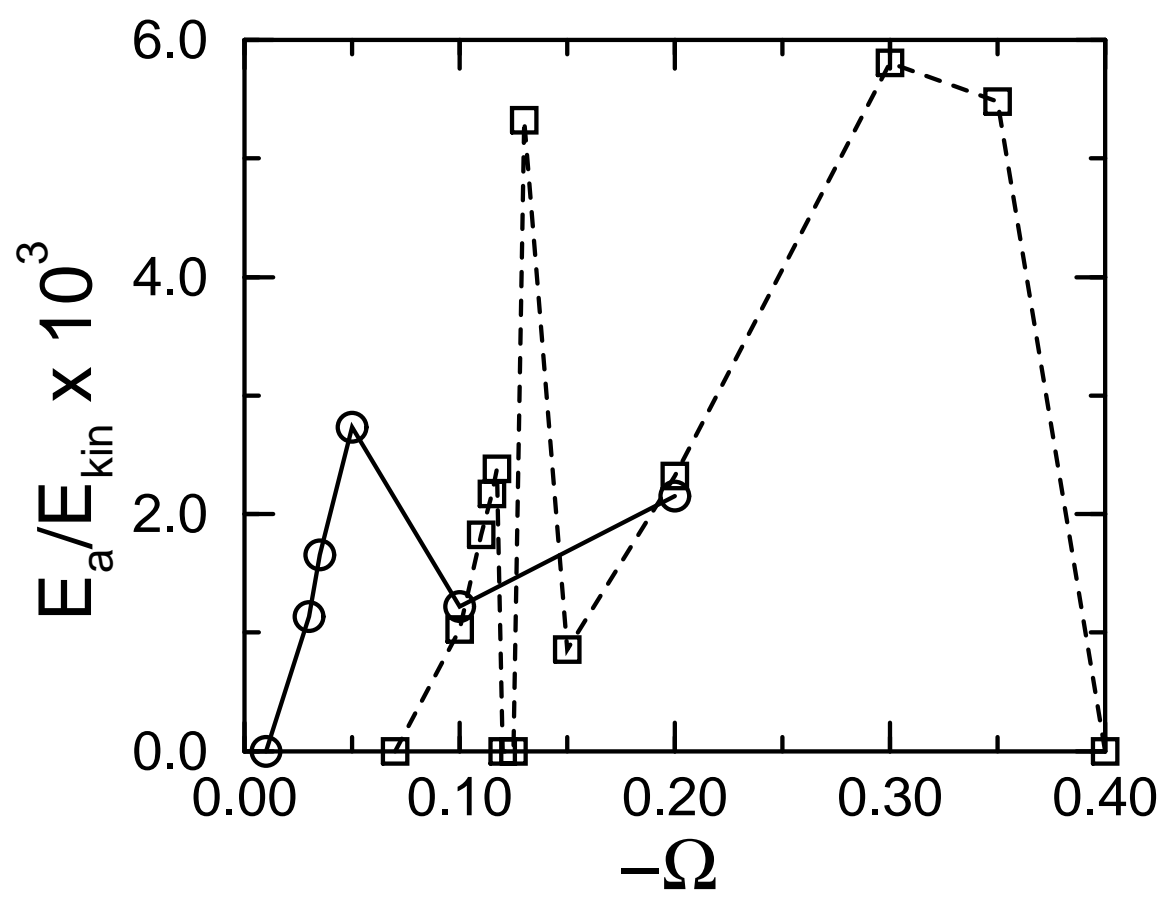

Figure 2.16: The ratio of the antisymmetric energy $E_{a}$ and the total kinetic energy $E_{k i n}$ as a function of the precession rate $\Omega$ for $E=10^{-4}, e=0.04, \eta=0.1, \alpha=30^{\circ}$ (circles) and $E=5 \times 10^{-4}, e=0, \eta=0.01, \alpha=60^{\circ}$ (squares).

On the other hand, there is no obvious reason why the instability should occur only in $\boldsymbol{u}_{a}$.

Figures 2.17 and 2.18 show indeed that for $E=10^{-4}, e=0.04, \alpha=30^{\circ}$ and $\Omega=$ -0.035 , the instability resides in modes with $m^{\prime}=6$ and 7 , the symmetric components containing more energy than the antisymmetric ones.

Away from the onset, an increasingly complex time dependence of the flow is observed and the main peak, in spectra like shown in figures 2.10 and 2.17 , becomes broader. Increasing $|\boldsymbol{\Omega}|$ or $E$ generally shifts the energy content to lower $m^{\prime}$. 


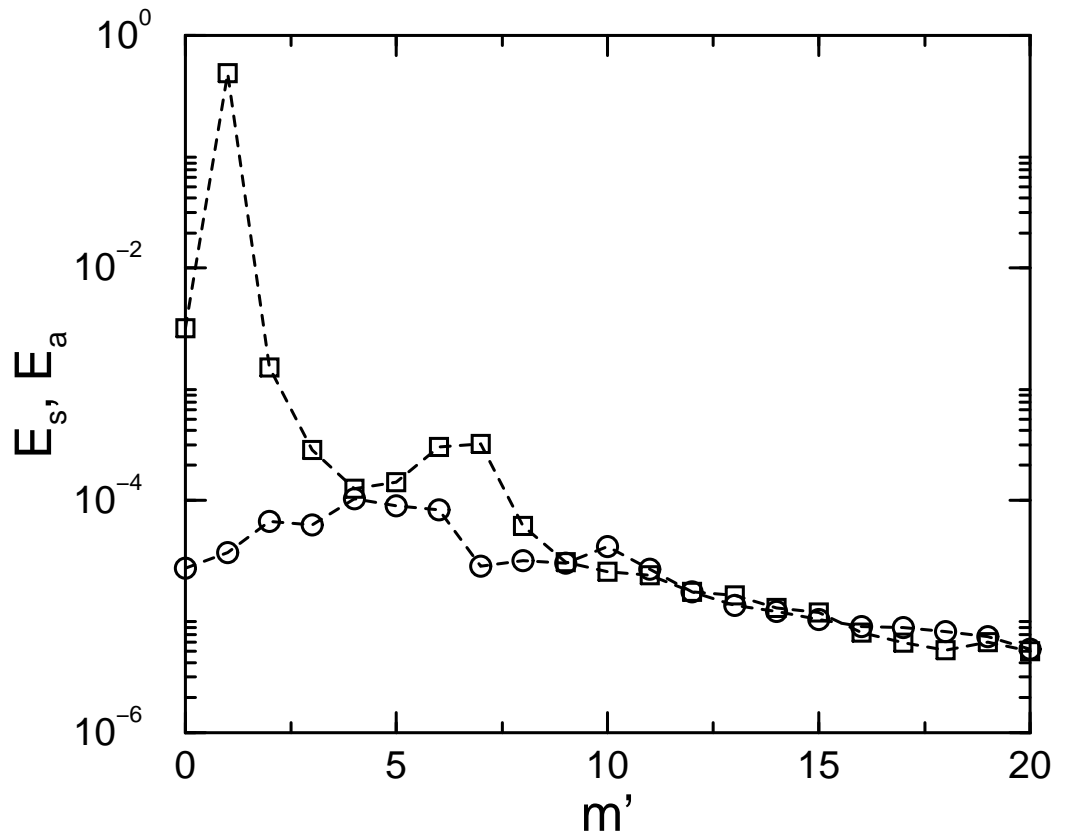

Figure 2.17: Energy contained in the modes with wavenumber $m^{\prime}$ as a function of $m^{\prime}$ for $\eta=0.1, e=0.04, \alpha=30^{\circ}, \Omega=-0.035$ and $E=10^{-4}$. Antisymmetric (circles) and symmetric (squares) contributions are shown separately.
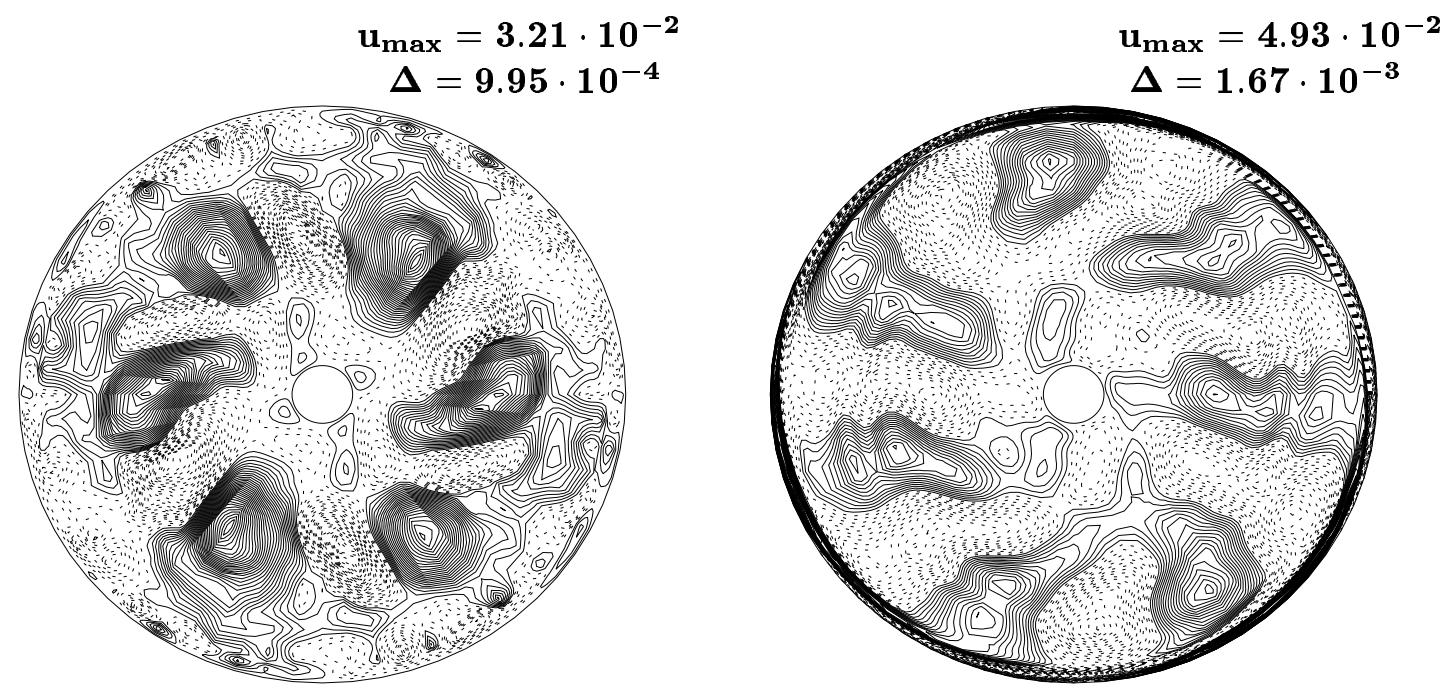

Figure 2.18: $u_{s r}$ (left) and $u_{s z^{\prime}}$ (right) in the plane perpendicular to $\boldsymbol{\omega}_{F}$ for the same parameters as in figure 2.17 . 
It is known from theoretical work (Pierrehumbert 1986, Gledzer \& Ponomarev 1992) that an inertial instability occurs in flows with elliptically deformed streamlines, and through related mechanisms in precession driven flow in ellipsoidal cavities (Kerswell 1993) [see chapter 4 for a detailed description of this kind of instabilities]. This mechanism is not effective in the sphere. Consequently, it is not possible to classify the detected instability within this already known theoretical frame, because the same type of instability has been observed in both spherical and ellipsoidal geometries. In the sphere, the Poincaré solution is a solid body rotation which is a stable flow, so that viscous corrections to the Poincaré flow must be responsible for the instabilities.

Incidentally, the criterion for the onset of instability given by Kerswell (1993) predicts stability for all the simulated flows. In spite of that, also the instability presented in this section manifests itself in the form of a triad resonance: two inertial waves are coupled by the underlying strain field.

A variation of the theoretical analyses mentioned above allows one to see that the bulk instability is due to flow components with wavenumber $m^{\prime}=1$. Following the presentations given by Gledzer \& Ponomarev (1992) and Kerswell (1993), consider the inertial mode problem posed by (2.48) and (2.49) appropriately linearized if $\boldsymbol{u}_{\boldsymbol{s}}$ is a solid body rotation plus a perturbation of wavenumber $m^{\prime}=1$. The interaction integrals in the perturbation expansion are different from zero only if they couple two eigenmodes of the unperturbed problem with time and azimuthal dependencies given by $e^{i\left(m_{a}^{\prime} \varphi^{\prime}-\omega_{a} t\right)}$ and $e^{i\left(m_{b}^{\prime} \varphi^{\prime}-\omega_{b} t\right)}$ with: $\left|m_{a}^{\prime}-m_{b}^{\prime}\right|=1$. Since the basic state has no time dependence in the chosen frame of reference, the resonance condition which has to be satisfied by the frequencies is: $\omega_{a}=\omega_{b}$. The instability thus consists of at least one pair of inertial modes with wavenumbers differing by one and nearly equal frequencies. For the examples reported in table 2.4, one sees that these conditions are fulfilled, with two pairs of resonant modes being excited in one case.

Experimental evidence (section 1.4) suggests that the axisymmetric internal cylindrical shear layers (associated with the $m^{\prime}=0$ deviations from the Poincaré solution) cause an instability even though the employed visualization techniques cannot ascertain whether these shear layers merely act as tracers or whether they actually trigger an instability. It seems highly unlikely that the instabilities presented here would be driven by an $m^{\prime}=$ 0 disturbance of the basic flow which could excite a single inertial mode of arbitrary wavenumber and frequency. Moreover, in all the simulations performed, the $m^{\prime}=1$ deviations from a flow with uniform vorticity outweight the $m^{\prime}=0$ deviations. However, decreasing the Ekman number, the viscous corrections associated to the $m^{\prime}=1$ deviations diminish, whereas the axisymmetric shear layer connecting the critical latitudes becomes more and more singular. An instability of that shear layer is thus plausible at low E.

Following Hide \& Titman (see section 1.3), one can determine the velocity difference at the two sides of the most pronounced shear layer associated with the $m^{\prime}=1$ component and construct a Rossby number from it. It turns out that this Rossby number increases with decreasing the Ekman number, so that the mechanism related to the $m^{\prime}=1$ components should remain effective even at the lower Ekman numbers at which the axisymmetric shear layer possibly becomes unstable, too. 
In conclusion: even if the strongest departures from a solution with constant vorticity are in the toroidal components of wavenumber 0 and 1 with respect to the fluid axis, only the instabilities triggered by the wavenumber $m^{\prime}=1$ deviations have actually been observed. Since the excited flow fills a large fraction of the fluid volume, the origin of the instability cannot be traced back to a more particular feature of the velocity field, as for example the critical latitudes. None of the phenomena observed by Malkus in his second experiment, i.e. a sudden transition with an abrupt increase of the driving torque as well as hysteresis (see section 1.4), have been seen in the simulations. On the contrary, the motions excited by the instability always accounted for only a small fraction of the total energy (see table 2.2).

\subsubsection{Ekman layer instability}

Independently of the dynamics of the bulk fluid, the Ekman layers may become unstable. This is demonstrated in figure 2.19 which shows as a function of radius the density $\epsilon_{a}(r)$ of the energy contained in $\boldsymbol{u}_{a}$ averaged over spherical surfaces:

$$
\epsilon_{a}(r)=\frac{1}{4 \pi} \int_{0}^{\pi} d \vartheta \sin \vartheta \int_{0}^{2 \pi} d \varphi \frac{1}{2} \boldsymbol{u}_{a}^{2}
$$

Three different situations are represented in figure 2.19. Either only the bulk becomes unstable in the manner already described, or only the boundary, or both. One instability does not seem to affect the other. The parameters for which the boundary layer instability has been observed are marked in table 2.2.

In order to find a criterion for the onset of this kind of instability, the procedure adopted by Tatro and Mollö-Christensen in their experiments has been followed (see section 1.2).

The distance from the boundary at which the absolute value of the radial velocity (averaged over the corresponding spheroidal surface) reaches a maximum is used as the boundary layer thickness. The layer thickness is approximately $1.4 \times E^{1 / 2}$ in all cases. Based on this thickness and the maximum tangential velocity $v_{h}$ at the edge of the boundary layer, the Reynolds numbers listed in table 2.2 have been computed. The Rossby numbers have been estimated as:

$$
v_{h}[2(1+\Omega \cos \alpha)]^{-1}
$$

The critical Reynolds number lies somewhere in between 50 and 100, which falls into the range quoted by Tatro \& Möllo-Christensen (1967). As general criterion, it has been deduced that the boundary layer becomes certainly unstable, independently of the bulk of the fluid, if its Reynolds number is larger than 100.

Although a systematic investigation of the critical parameters for the onset of this instability is beyond the scope of the present research, a qualitative comparison with the previous works can be made as concerns the shape of the instability. Figures 2.20 and 2.21 give an impression of the unstable boundary layer flow. The pictures show very clearly that, in agreement with the findings of Lilly (1966), Leibovich \& Lele (1985), Des- 


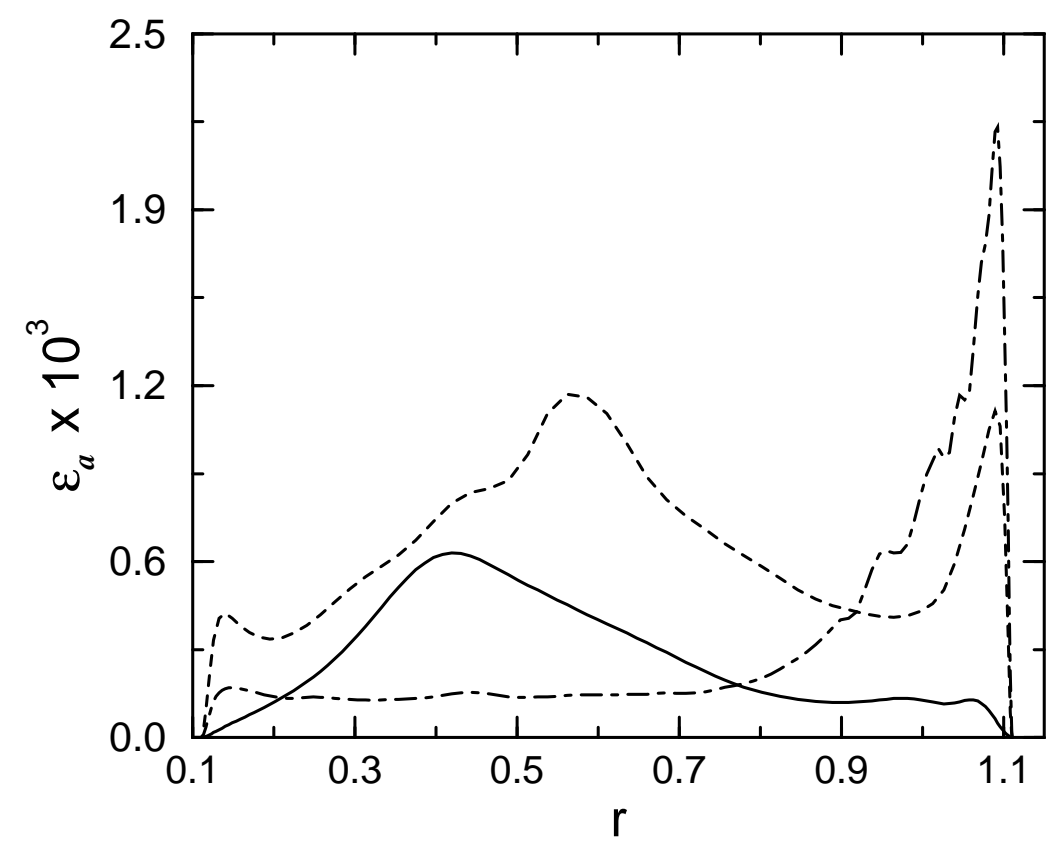

Figure 2.19: The energy density $\epsilon_{a}$ of the antisymmetric components (equ.(2.50)) as a function of $r$ for $E=5 \times 10^{-4}, e=0.1, \alpha=80^{\circ}, \Omega=-0.08$ (solid), $E=10^{-4}, e=0.04$, $\alpha=30^{\circ}, \Omega=-0.05$ (dashed), and $E=10^{-4}, e=0.1, \alpha=80^{\circ}, \Omega=-0.08$ (dot dashed). The values for the last curve have been divided by ten. $\eta=0.1$ for all cases.

jardins, Dormy and Grenier (2001) (DDG), the Ekman flow is unstable to two dimensional disturbances and reveals the two different situations summarized in figure 1.5 section 1.2.

When the critical band for the occurrence of the instability extends near the pole (as it is shown in figure 2.20), the axis of the two dimensional rolls forms an angle with the $e_{\varphi}$ direction and the disturbances propagate towards the pole away from the equator.

Vice versa, if a critical band is found to extend in the equatorial region, the instability is aligned with $e_{\vartheta}$ (north-south direction) and propagates in the azimuthal direction. Figure 2.21 shows that, for small Ekman numbers $\left(E=2 \times 10^{-5}\right)$, a critical latitude appears at approximately 45 degrees and generates small-scales rolls emanating from the equatorial region. This is in agreement with the results of DDG for geophysically relevant values of the parameters (see section 1.2). Note that in both the examples presented here, the instability develops around the rotation axis of the fluid. For the sake of completeness, figure 2.22 shows the kind of flows which can originate within a stable Ekman boundary layer.

The comparatively small lateral length scales of the motions excited by the boundary layer instability become difficult to resolve at more extreme parameters. The development of this instability and ensuing numerical instabilities turn out to be the most serious 

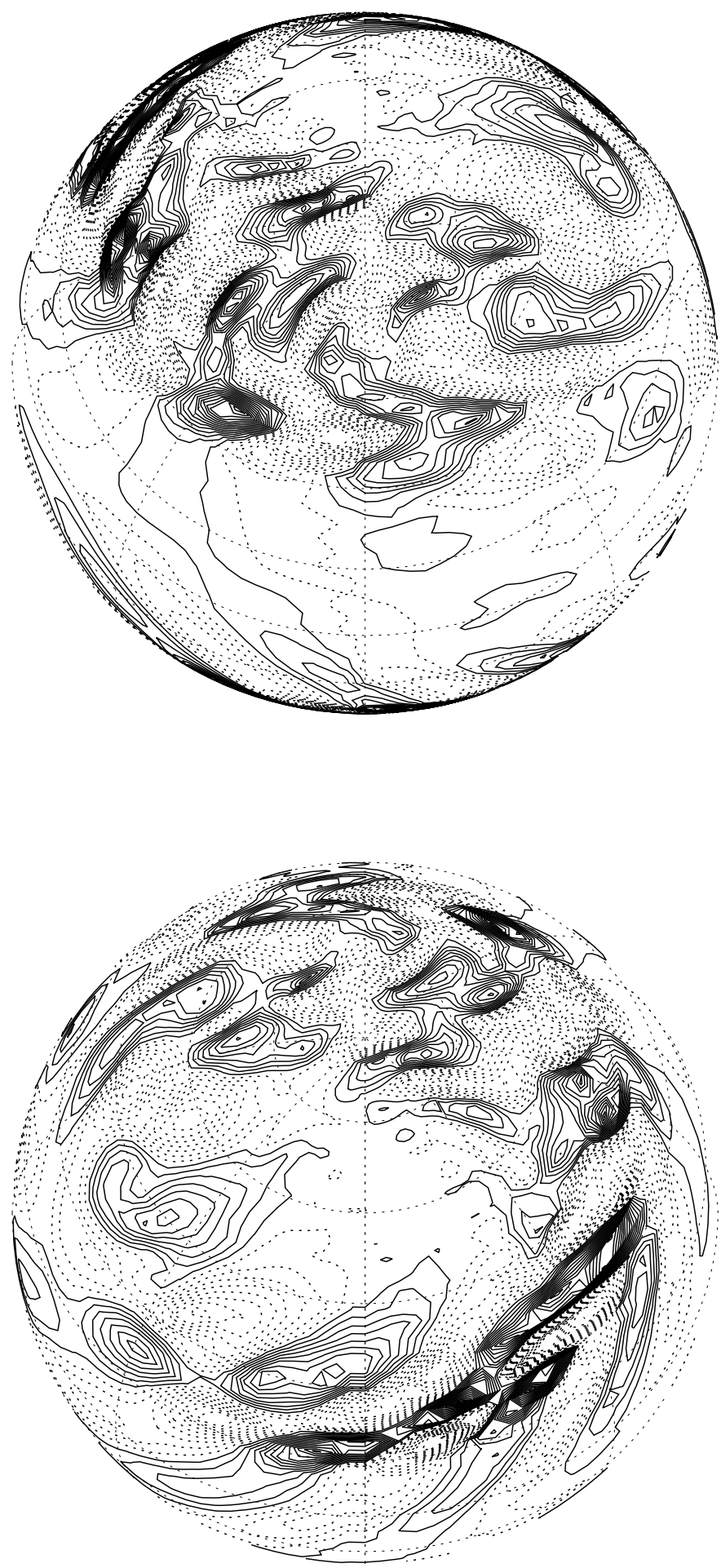

Figure 2.20: $u_{a r}$ on a surface separated by 0.015 from the outer boundary for $e=0.04$, $\eta=0.1, \alpha=30^{\circ}, E=10^{-4}$ and $\Omega=-0.03$. The top figure is centered around $\varphi=0^{\circ}$, whereas the bottom figure is centered around $\varphi=180^{\circ}$. The fluid axis is located at $\vartheta \simeq 31^{\circ}$ and $\varphi \simeq 164^{\circ}$. 

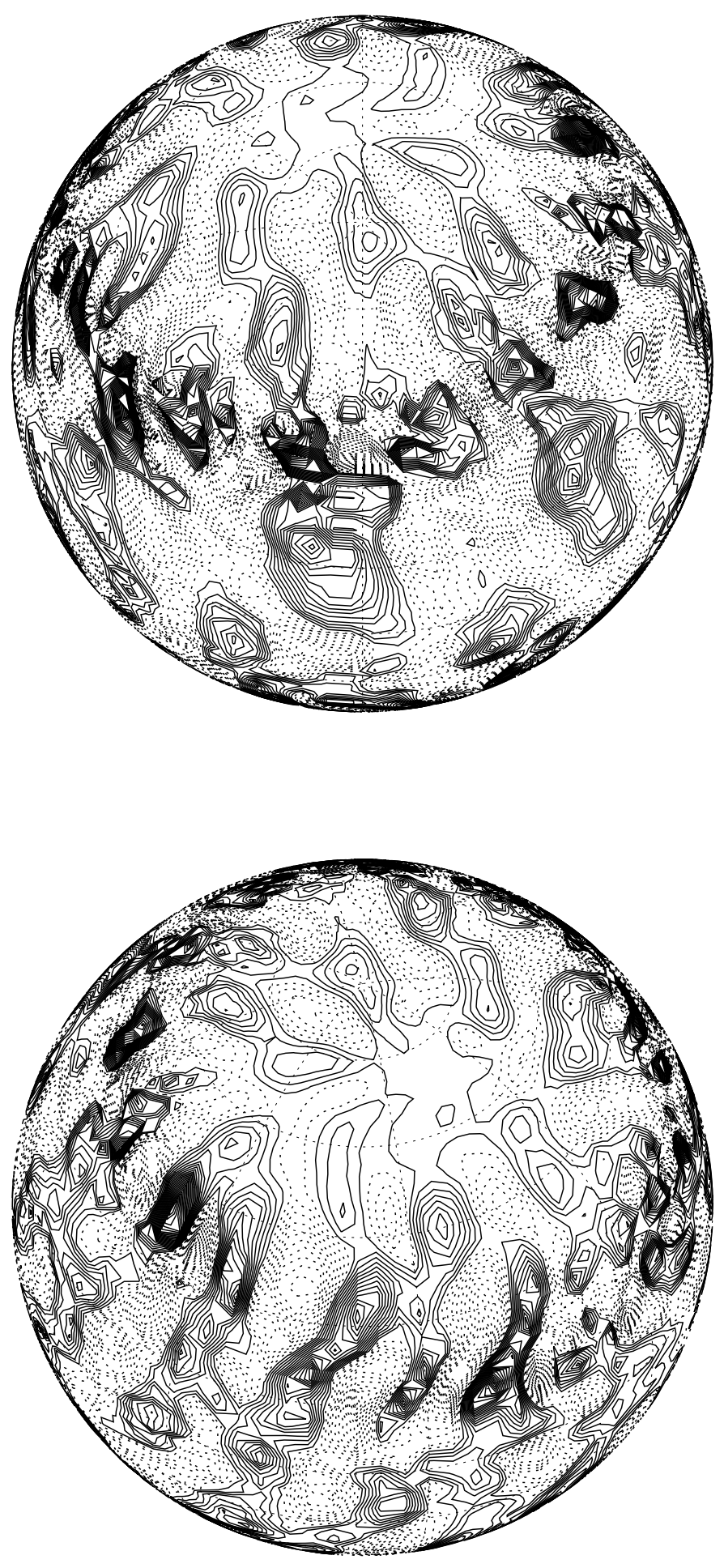

Figure 2.21: $u_{a r}$ on a surface separated by 0.0069 from the outer boundary for $e=0.04$, $\eta=0.1, \alpha=30^{\circ}, E=2 \times 10^{-5}$ and $\Omega=-0.018$. The top figure is centered around $\varphi=180^{\circ}$, whereas the bottom figure is centered around $\varphi=-11^{\circ}$. The fluid axis is located at $\vartheta \simeq 20^{\circ}$ and $\varphi \simeq 11^{\circ}$. 


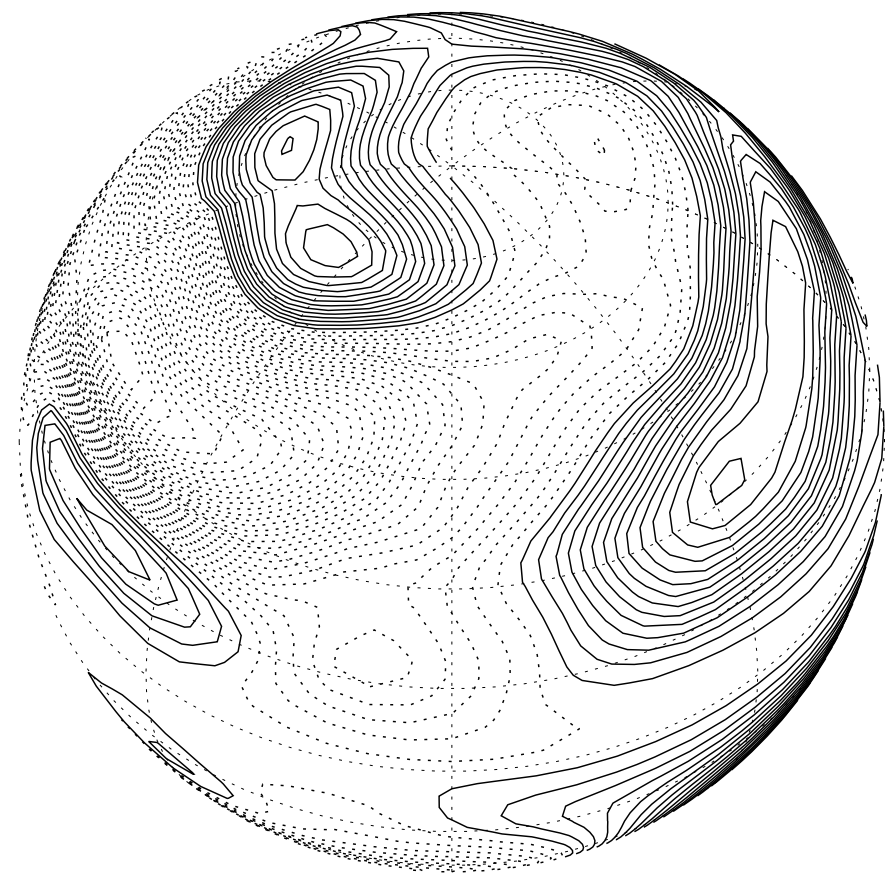

Figure 2.22: $u_{a r}$ on a surface separated by 0.03 from the outer boundary for $e=0.1$, $\eta=0.1, \alpha=80^{\circ}, E=5 \times 10^{-4}$ and $\Omega=-0.08$.

obstacle on the route to high precession rates at low $E$. 


\section{Chapter 3}

\section{Precession driven dynamo}

\subsection{Introduction}

It was believed for a long time that the magnetic field of the Earth could be explained in terms of permanent magnetisation.

It is now well known that the temperature of the Earth's interior is above the critical Curie temperature at which ferromagnetic materials lose their 'permanent' magnetisation.

The discovery (by seismological observations) that a large fraction of the Earth's interior is in a liquid state revealed profoundly relevant to the problem concerning the origin of the Earth's magnetic field.

Nowadays it is accepted that the magnetic field of the Earth is generated by motions in its liquid part.

The mathematical problem describing the generation of magnetic fields by motions in an electrically conducting fluid (conversion of mechanical energy into magnetic energy) is called the dynamo problem.

The dynamo principle has been used since a long time in technical processes.

The simplest example of a technical dynamo is the 'homopolar' disk dynamo (Bullard, 1955) shown in figure 3.1 .

A solid copper disk rotates about its axis with angular velocity $\boldsymbol{\omega}_{D}$ in a weak initial magnetic field $\boldsymbol{B}_{o}$. The field induces an electromotive force between the axis and the rim of the disk that can be used to drive a current $I$ in the circuit shown in figure 3.1.

The equation for $I(t)$ is then:

$$
L \frac{d I}{d t}+R I=M \omega_{D} I
$$

where: $M=M_{o} / 2 \pi$ with $M_{o}$ the mutual inductance between the circuit and the rim of the disk, $L$ and $R$ are the self-inductance and resistance of the complete circuit.

If:

$$
\omega_{D}>R / M
$$

the current grows exponentially. 


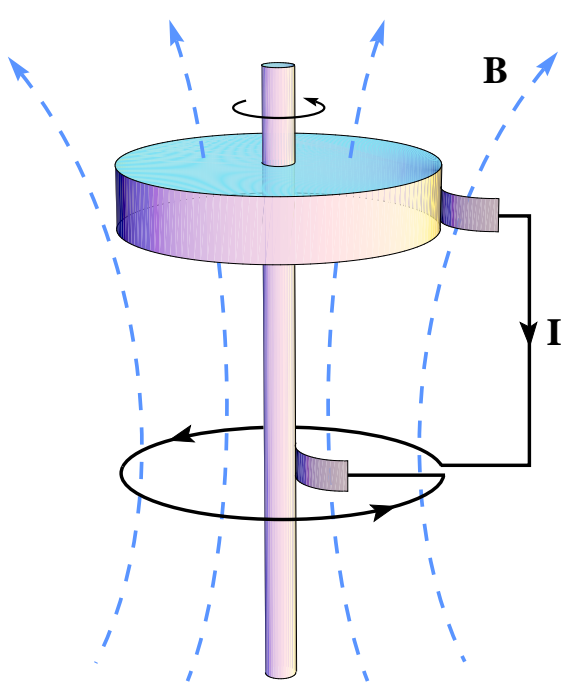

Figure 3.1: The homopolar disk dynamo. Note that the twist in the wire which carries the current $I(t)$ must be in the same sense of the rotation of the solid disk.

Provided that the winding of the circuit has the appropriate sense (see figure 3.1), the field $\boldsymbol{B}$ generated by the current has the same direction as the initial field $\boldsymbol{B}_{o}$, but higher modulus.

A self-excited dynamo is then obtained. This example, which helps to understand the general mechanism of magnetic field generation, differs nevertheless from the conducting fluid situation in that the current is constrained by the twisted geometry to follow a very special path.

These geometrical constraints are not available in the singly-connected planetary cores.

In other respects the fluid dynamo is rather similar to the disk dynamo (Busse, 1978).

Some interesting properties that the dynamo equation possesses, collectively named 'anti-dynamo' or 'negative' theorems, are summarized in the following.

(a) Cowling's theorem (1934) states that a steady axisymmetric magnetic field cannot be maintained by axisymmetric motions. The work of Braginsky $(1965,1975)$ has later shown that arbitrary small deviations from axisymmetry are sufficient to generate magnetic fields if the magnetic Reynolds number is high enough.

(b) The toroidal theorem, found by Elsasser (1946) and proved rigorously by Bullard \& Gellman (1954), states that purely toroidal velocity fields can sustain only decaying magnetic fields. A toroidal velocity field may lead to an initial increase of the magnetic energy, but ultimately $\boldsymbol{B}$ must decay because of the unbalanced ohmic dissipation of the poloidal component of the magnetic field. 
(c) A planar two dimensional motion cannot be a dynamo if the flow occurs in a conductor filling the entire space or if vacuum boundaries are parallel to the planes of the flow.

\subsection{Mathematical formulation and numerical meth- ods}

The magnetic induction equation that governs the evolution of a magnetic field $\boldsymbol{B}$ in a conducting fluid moving with velocity $\boldsymbol{u}$, derived from Ohm's law and Maxwell's equations in the magnetohydrodynamic approximation in which the displacement current is neglected, is written as follows (in dimensional form):

$$
\frac{\partial \boldsymbol{B}}{\partial t}+\nabla \times(\boldsymbol{B} \times \boldsymbol{u})=\frac{1}{\mu \sigma} \nabla^{2} \boldsymbol{B}
$$

with: $\nabla \cdot \boldsymbol{B}=0$.

The inverse of the product of the electrical conductivity $\sigma$ and the permeability $\mu$ is called the magnetic diffusivity $\lambda$. In deriving (3.1) it has been assumed that $\lambda$ is constant.

In order to describe the dynamo effect in an electrically conducting fluid, the NavierStokes equation has to be considered in conjunction with the magnetic equation (3.1).

The coupling between the two equations through the Lorentz force introduces a nonlinearity in the full 'magnetohydrodynamic dynamo problem'.

In the following a fluid of constant density $\varrho$ with kinematic viscosity $\nu$ and conductivity $\sigma$ in a spherical shell of gap width $d$ which rotates about its axis of symmetry (with angular frequency $\boldsymbol{\omega}_{D}$ ) and whose rotation axis is executing precessional motion will be considered.

Units of length, time and magnetic field strength are chosen as: $d, 1 / \omega_{D}$ and $\left(\varrho \mu_{o}\right)^{1 / 2} d \omega_{D}$, respectively. $\mu_{o}$ is the vacuum magnetic permeability.

The non-dimensional Navier-Stokes and magnetic induction equations read in the mantle frame:

$$
\begin{aligned}
& \frac{\partial \boldsymbol{u}}{\partial t}+(\nabla \times \boldsymbol{u}) \times \boldsymbol{u}+2(\hat{\boldsymbol{z}}+\boldsymbol{\Omega}) \times \boldsymbol{u}= \\
& -\nabla \Phi+E \nabla^{2} \boldsymbol{u}-(\boldsymbol{\Omega} \times \hat{\boldsymbol{z}}) \times \boldsymbol{r}+(\nabla \times \boldsymbol{B}) \times \boldsymbol{B} \\
& \frac{\partial \boldsymbol{B}}{\partial t}+\nabla \times(\boldsymbol{B} \times \boldsymbol{u})=\frac{E}{P_{m}} \nabla^{2} \boldsymbol{B}
\end{aligned}
$$

where the Ekman number is defined as: $E=\nu / d^{2} \omega_{D}$ and the magnetic Prandtl number as: $P_{m}=\mu \sigma \nu$.

$\Phi$ stands for the reduced pressure which is irrelevant here because only the curl of (3.2) will be used.

The precession axis $\hat{\boldsymbol{\Omega}}$ forms an angle $\alpha(0<\alpha<\pi / 2)$ with the axis of symmetry of the shell ( $z$-axis) and is time dependent in the mantle frame: 


$$
\hat{\mathbf{\Omega}}=\sin \alpha \cos t \hat{\boldsymbol{x}}-\sin \alpha \sin t \hat{\boldsymbol{y}}+\cos \alpha \hat{\boldsymbol{z}}
$$

The no-slip boundary conditions for the velocity field require that: $\boldsymbol{u}=0$ at $r=r_{i}, r_{o}$, whereas it has been assumed that $\boldsymbol{B}$ matches a vacuum field at the inner and outer boundaries ([70]).

The solenoidal velocity and magnetic fields have been expressed in terms of poloidal and toroidal scalars:

$$
\begin{aligned}
& \boldsymbol{u}=\nabla \times \nabla \times(\Phi \hat{\boldsymbol{r}})+\nabla \times(\Psi \hat{\boldsymbol{r}}) \\
& \boldsymbol{B}=\nabla \times \nabla \times(g \hat{\boldsymbol{r}})+\nabla \times(h \hat{\boldsymbol{r}})
\end{aligned}
$$

which are then decomposed into radial and angular parts:

$$
\begin{aligned}
& \Phi=r \sum_{l=1}^{\infty} \sum_{m=-l}^{l} V_{l}^{m}(r, t) P_{l}^{m}(\cos \vartheta) e^{i m \varphi} \\
& \Psi=r^{2} \sum_{l=1}^{\infty} \sum_{m=-l}^{l} W_{l}^{m}(r, t) P_{l}^{m}(\cos \vartheta) e^{i m \varphi} \\
& g=\sum_{l=1}^{\infty} \sum_{m=-l}^{l} G_{l}^{m}(r, t) P_{l}^{m}(\cos \vartheta) e^{i m \varphi} \\
& h=\sum_{l=1}^{\infty} \sum_{m=-l}^{l} H_{l}^{m}(r, t) P_{l}^{m}(\cos \vartheta) e^{i m \varphi}
\end{aligned}
$$

$P_{l}^{m}(\cos \vartheta)$ denote associated Legendre functions.

Operating with $\hat{\boldsymbol{r}} \cdot \nabla \times$ and $\hat{\boldsymbol{r}} \cdot \nabla \times \nabla \times$ on (3.2) and with $\hat{\boldsymbol{r}} \cdot$ and $\hat{\boldsymbol{r}} \cdot \nabla \times$ on (3.3) one obtains:

$$
\begin{gathered}
\frac{\partial}{\partial t} \mathcal{D}_{l} V_{l}^{m}-E \cdot \mathcal{D}_{l}^{2} V_{l}^{m}=\frac{r}{l(l+1)}\left[\hat{\boldsymbol{r}} \cdot \nabla \times \nabla \times\left\{\left(\boldsymbol{\Omega}^{\prime} \times \boldsymbol{u}\right)-\right.\right. \\
(\nabla \times \boldsymbol{B}) \times \boldsymbol{B}\}]_{l}^{m} \\
\frac{\partial}{\partial t} W_{l}^{m}-E\left(\frac{\partial^{2}}{\partial r^{2}}+\frac{4}{r} \frac{\partial}{\partial r}+\frac{2-l(l+1)}{r^{2}}\right) W_{l}^{m}=-\frac{1}{l(l+1)} \\
{\left[\hat{\boldsymbol{r}} \cdot \nabla \times\left\{\boldsymbol{\Omega}^{\prime} \times \boldsymbol{u}-(\nabla \times \boldsymbol{B}) \times \boldsymbol{B}\right\}\right]_{l}^{m}+[f]_{l}^{m}} \\
\frac{\partial}{\partial t} G_{l}^{m}-\frac{E}{P_{m}}\left(\frac{\partial^{2}}{\partial r^{2}}-\frac{l(l+1)}{r^{2}}\right) G_{l}^{m}=-\frac{r^{2}}{l(l+1)} \cdot \\
{[\hat{\boldsymbol{r}} \cdot \nabla \times(\boldsymbol{B} \times \boldsymbol{u})]_{l}^{m}}
\end{gathered}
$$




$$
\begin{aligned}
\frac{\partial}{\partial t} H_{l}^{m}- & \frac{E}{P_{m}}\left(\frac{\partial^{2}}{\partial r^{2}}-\frac{l(l+1)}{r^{2}}\right) H_{l}^{m}=-\frac{r^{2}}{l(l+1)} \\
& {[\hat{\boldsymbol{r}} \cdot \nabla \times \nabla \times(\boldsymbol{B} \times \boldsymbol{u})]_{l}^{m} }
\end{aligned}
$$

with: $\boldsymbol{\Omega}^{\prime}=\nabla \times \boldsymbol{u}+2(\hat{\boldsymbol{z}}+\boldsymbol{\Omega}), \quad \mathcal{D}_{l}=\frac{\partial^{2}}{\partial r^{2}}+\frac{2}{r} \frac{\partial}{\partial r}-\frac{l(l+1)}{r^{2}}$ and $f=\frac{1}{2} \Omega \sin \alpha\left[i P_{1}^{1} e^{i(\varphi+t)}+2 i P_{1}^{-1} e^{-i(\varphi+t)}\right]$.

[]$_{l}^{m}$ denotes the $l, m$-component of the quantity in the square bracket.

The no-slip boundary conditions for the velocity field read in terms of poloidal and toroidal scalars:

$$
V_{l}^{m}=\frac{\partial V_{l}^{m}}{\partial r}=W_{l}^{m}=0 \quad \text { at } r=r_{i}, r_{o}
$$

The boundary conditions for the magnetic field require that $\boldsymbol{B}$ matches a potential field outside the fluid volume. In the vacuum region:

$$
\begin{gathered}
\boldsymbol{B}=\nabla \times \nabla \times\left(g_{v a c} \hat{\boldsymbol{r}}\right)+\nabla \times\left(h_{v a c} \hat{\boldsymbol{r}}\right) \\
\nabla \times \boldsymbol{B}=0
\end{gathered}
$$

From this latter relation follows:

$$
\begin{gathered}
h_{v a c}=0 \\
{\left[g_{v a c}\right]_{l}^{m}=A_{l}^{m} r^{-l}+B_{l}^{m} r^{l+1}}
\end{gathered}
$$

Regularity at the origin requires that $A_{l}^{m}=0$ in the inner core, whereas finite $\boldsymbol{B}$ requires that $B_{l}^{m}=0$ in the outer region.

$\boldsymbol{B}$ must be continuous, therefore the poloidal and toroidal scalars and the radial derivative of the poloidal scalar are continuous.

It follows that:

at the outer boundary

$$
\begin{aligned}
H_{l}^{m}\left(r_{o}\right) & =0 \\
G_{l}^{m}\left(r_{o}\right) & =A_{l}^{m} r_{o}^{-l} \\
\frac{\partial}{\partial r} G_{l}^{m}\left(r_{o}\right) & =-l A_{l}^{m} r_{o}^{-l-1}
\end{aligned}
$$

at the inner boundary

$$
\begin{aligned}
H_{l}^{m}\left(r_{i}\right) & =0 \\
G_{l}^{m}\left(r_{i}\right) & =B_{l}^{m} r_{i}^{l+1} \\
\frac{\partial}{\partial r} G_{l}^{m}\left(r_{i}\right) & =(l+1) B_{l}^{m} r_{i}^{l}
\end{aligned}
$$

Finally, one can write the boundary conditions for $H_{l}^{m}$ and $G_{l}^{m}$ as: 


$$
\begin{aligned}
H_{l}^{m}=\left(\frac{\partial}{\partial r}-\frac{l+1}{r}\right) G_{l}^{m}=0 & \text { at } r=r_{i} \\
H_{l}^{m}=\left(\frac{\partial}{\partial r}+\frac{l}{r}\right) G_{l}^{m}=0 & \text { at } r=r_{o}
\end{aligned}
$$

The numerical methods used to solve equations (3.7),(3.8) have been illustrated in details in section 2.2. Their extension in order to include the magnetic field equations (3.9) and (3.10) is straightforward ([70]).

\subsection{Kinematic dynamo problem}

The simplest dynamo models, which can be considered in order to decide whether a fluid flow acts as a dynamo, ignore dynamical influences on $\boldsymbol{u}$ such as the Lorentz force.

In the 'kinematic dynamo problem' the velocity field $\boldsymbol{u}$ is prescribed and a primary aim is to investigate the conditions under which growing solutions $\boldsymbol{B}$ of (3.3) exist.

In the case of time-independent fields $\boldsymbol{u}$ and assuming an exponential time dependence for the magnetic field

$$
\boldsymbol{B}=\boldsymbol{B}_{o} e^{p t}
$$

a dynamo process occurs if a solution $\boldsymbol{B}$ of (3.3) exists for which the eigenvalue $p$ has a positive real part.

Writing the total magnetic energy $E_{B}(t)$ as:

$$
E_{B}(t)=\frac{1}{2} \int_{V_{\infty}} \boldsymbol{B}^{2} d V
$$

where $V_{\infty}$ represents the whole space, the following definition can be given: the velocity field $\boldsymbol{u}$ acts as a dynamo if $E_{B}(t) \not \rightarrow 0$ as $t \rightarrow \infty$, i.e. if it successfully counteracts the erosive action of ohmic dissipation [Moffatt, 1978].

An indefinitely growing magnetic energy describes clearly an unphysical situation.

In reality, as the magnetic field grows, the Lorentz force modifies the velocity in such a way that a final equilibrium state for the magnetic energy is reached.

Therefore, the kinematic dynamo problem describes the dynamo process in its initial stages.

When the amplitude of the magnetic field reaches a value such that the Lorentz force is not anymore negligible, the full magnetohydrodynamic dynamo problem (presented in section 3.2) must be considered.

The kinematic and magnetohydrodynamic dynamo problems have the same mathematical relationship as the linear and nonlinear problems in the context of hydrodynamic instability [Busse, 1978]. 


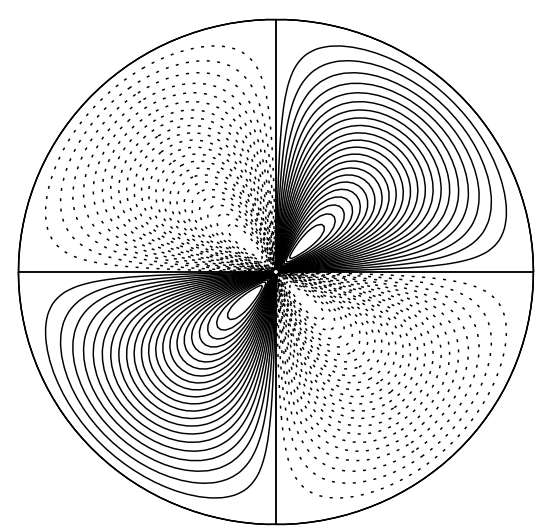

Figure 3.2: The simple rolls flow given by equation (3.13).

The dynamo action can be considered as a kind of magnetohydrodynamic instability in which the motion of a conducting fluid becomes unstable to a growing disturbance characterized by the appearance of a new physical quantity: the magnetic field.

In the following only the kinematic dynamo problem in a spherical shell will be addressed in order to decide whether the velocity fields introduced in section 2.4 act as a dynamo.

The possibility that purely laminar solutions in spherical and ellipsoidal cavities can generate a dynamo action has been already investigated in details by Tilgner (1998).

The laminar flows found in spherical geometries (Tilgner, 2001) are nearly toroidal. Only viscous corrections to the solid-body rotation add a poloidal component. Several tests have been made but no growing magnetic field appeared.

In an ellipsoid the inviscid solution is not anymore purely toroidal, evading therefore the toroidal anti-dynamo theorem reported in section 3.1 .

However, the streamlines lie on planes. A further anti-dynamo theorem states that a plane two dimensional motion cannot be a dynamo if the flow occurs in a conductor filling the entire space or if vacuum boundaries are parallel to the planes of the flow.

This last statement is not satisfied by the Poincaré flow.

In spite of that, numerical calculations performed by Tilgner (1998) led to the conclusion that is unlikely that such kind of flows can become a kinematic dynamo.

These results motivate the present research in that more complex unstable flows can have better chance of generating a magnetic field.

A preliminary study has been carried out choosing a particularly simple stationary velocity model which has been seen by Dudley \& James (1989) to sustain growing magnetic fields.

The velocity field considered, consisting of two very simple axisymmetric rolls (see figure 3.2 ), can be written as:

$$
\boldsymbol{u}=W_{2}^{0}(r)+\epsilon V_{2}^{0}(r)
$$




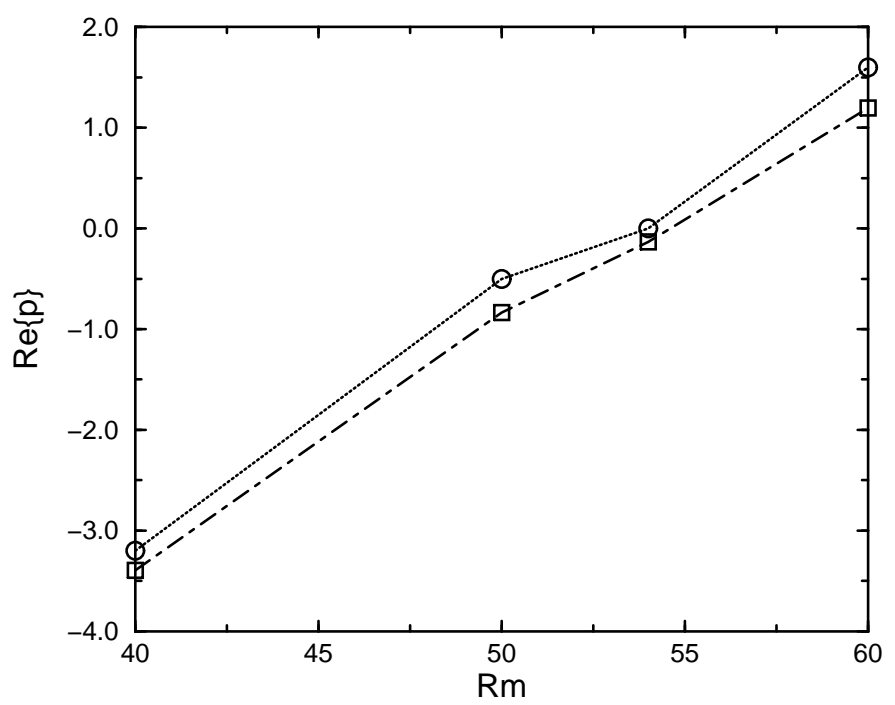

Figure 3.3: $\Re_{e}\{p\}\left(R_{m}\right)$ profiles for the simple rolls flow given by equation (3.13). Our numerical findings (squares) are compared with the Dudley \& James' results (circles).

where:

$$
\begin{gathered}
W_{2}^{0}(r)=\sin (\pi r) \\
V_{2}^{0}(r)=r \sin (\pi r)
\end{gathered}
$$

and $r \leq 1$ is the radius in the unit sphere.

$\epsilon$ is the poloidal to toroidal flow ratio which has to be of appropriate magnitude in order to obtain a non-decaying $\boldsymbol{B}$ solution.

Growing modes were not detected by Dudley \& James for $\epsilon=0.05,0.1$ but were for $\epsilon=0.14$, therefore, in the following, $\epsilon$ has been fixed at the value of 0.14 . Replacing the ratio $P_{m} / E$ in (3.3) with the magnetic Reynolds number $R_{m}$ and taking $P_{m}=1$, profiles of $p$ (see equation (3.11)) as a function of $R_{m}$ have been determined. A functioning dynamo for the purpose of this investigation is one with $\Re_{e}\{p\} \geq 0$. Figure 3.3 shows the obtained results (squares) compared with those presented by Dudley \& James (circles).

The two series of values are very close although a small deviation is evident. This discrepancy can be mainly ascribed to the difficulty in extracting the precise values from the graphs reported by these authors.

In spite of that, a steady state is clearly visible at $R_{m} \simeq 54$ and growing solutions exist when $R_{m}>54$.

This simple stationary velocity model, in addition to offer a good oppurtunity in testing the numerical code, reveals particularly useful in dispeling the somewhat prevalent belief 


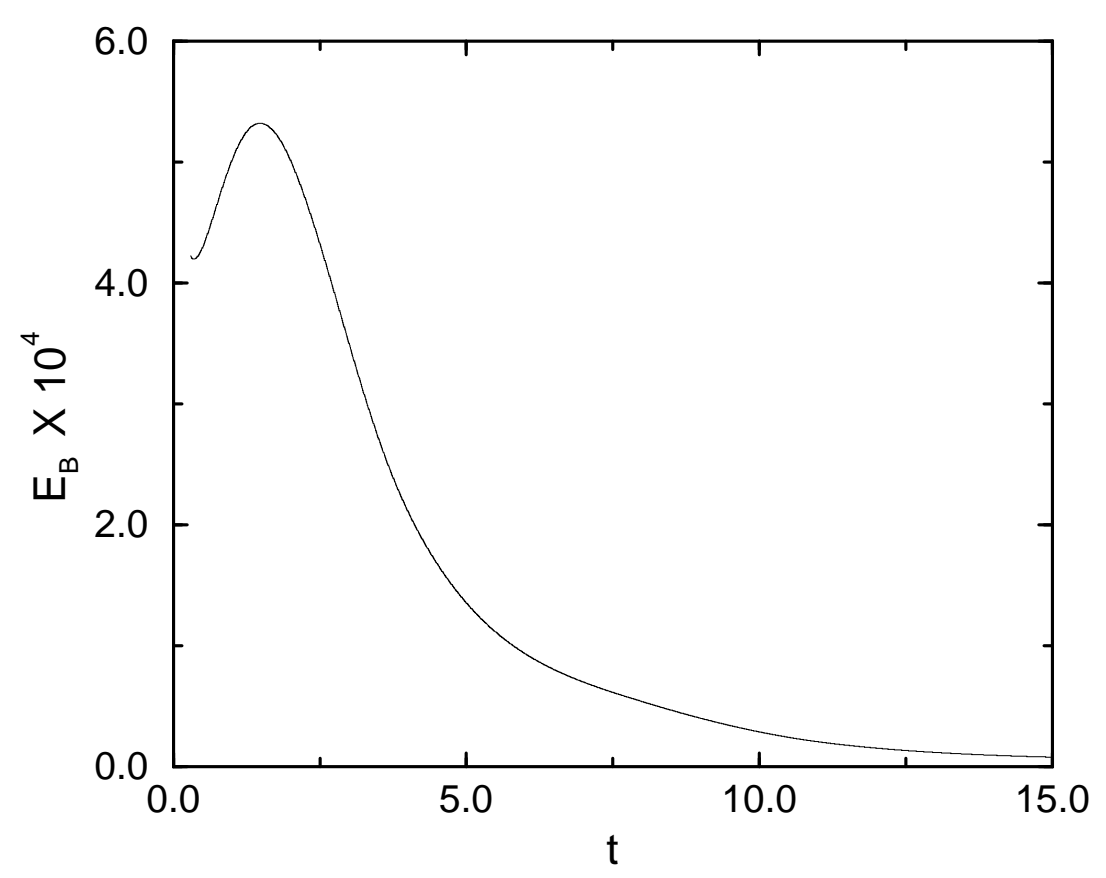

Figure 3.4: Time evolution of the magnetic energy given by (3.12) generated by the velocity field corresponding to the following parameters: $\eta=0.01, e=0, \alpha=60^{\circ}, \Omega=$ $-0.1, E=5 \times 10^{-4} . R_{m}=200$.

that only complex flows can support the dynamo action, provided that the magnetic Reynolds number and the poloidal to toroidal flow ratio are of appropriate magnitudes.

The simple roll flow introduced by Dudley \& James is not very different, in appearance, from the viscously unstable velocity field presented in section 2.4.

The kinematic precession-driven dynamo problem has been investigated for a fluid flow corresponding to the following parameters:

$$
\eta=0.01, e=0, \alpha=60^{\circ}, \Omega=-0.1 \text { and } E=5 \times 10^{-4}
$$

and sketched in figure 2.14 .

At this fixed Ekman number, the magnetic Prandtl number has been varied in order to inspect a range of magnetic Reynolds numbers as in figure 3.3.

Even though numerical difficulties prevented the exploration of a large parametric space, at a typical $P_{m}=0.1, R_{m}=200$ which is certainly not a small number.

Notwithstanding that, in the attempts at simulating a precession-driven dynamo made so far, the initial magnetic field got converted by interaction with the flow into a strong toroidal field which eventually decayed.

This feature is well illustrated in figure 3.4, which reproduces the time evolution of the 
magnetic energy (3.12) for $R_{m}=200$.

The energy contained in the unstable modes of the velocity field has always stayed small compared with the total energy and revealed not large enough for the dynamo action.

As Dudley \& James' velocity field suggests, the flow must be sufficiently strong if a magnetic response is to be excited. 


\section{Chapter 4}

\section{Inertial instabilities}

\subsection{Theoretical background}

The constant-vorticity Poincaré's solution for inviscid fluids, within a precessing spheroidal cavity, presented in section 2.1.1, is inertially unstable due to the strain which the bulk of the flow undergoes (Kerswell, 1993). This straining field consists of a boundary-induced 'elliptical' component, which elliptically distorts the streamlines and a precessional-induced 'shearing' component, which shears the centres of such ellipses in that the line joining these centres is not perpendicular to the plane of the streamlines (see figure 4.1). The elliptical component acts in the plane of the streamlines, while the 'shearing' component is perpendicular to this plane. Both these strains can couple two inertial waves, through a 'triad resonance' mechanism, when the difference in their frequencies coincides with one of the 'distortion' frequencies of the basic flow.

Poincaré's solution, given by equation (2.12), can be expressed in a more elegant coordinate independent form as follows (with respect to the precessing frame rotating at $\boldsymbol{\Omega}$ where the $z$-axis is chosen to coincide with the axis of the container):

$$
\boldsymbol{\omega}_{F}=\hat{\boldsymbol{z}}-\frac{2+\eta}{\eta+2(1+\eta) \boldsymbol{\Omega} \cdot \hat{\boldsymbol{z}}} \hat{\boldsymbol{z}} \times(\hat{\boldsymbol{z}} \times \mathbf{\Omega})
$$

where: $\eta=-1+1 /(1-e)^{2}$.

The full solution is a solid body rotation plus a potential flow which is necessary to accommodate the boundary condition:

$$
\boldsymbol{u}=\boldsymbol{\omega}_{F} \times \boldsymbol{r}+\nabla A
$$

with:

$$
A=\frac{\eta}{\eta+2(1+\eta) \boldsymbol{\Omega} \cdot \hat{\boldsymbol{z}}}(\boldsymbol{\Omega} \times \hat{\boldsymbol{z}} \cdot \boldsymbol{r})(\hat{\boldsymbol{z}} \cdot \boldsymbol{r})
$$

The flow is two-dimensional in planes inclined at: 


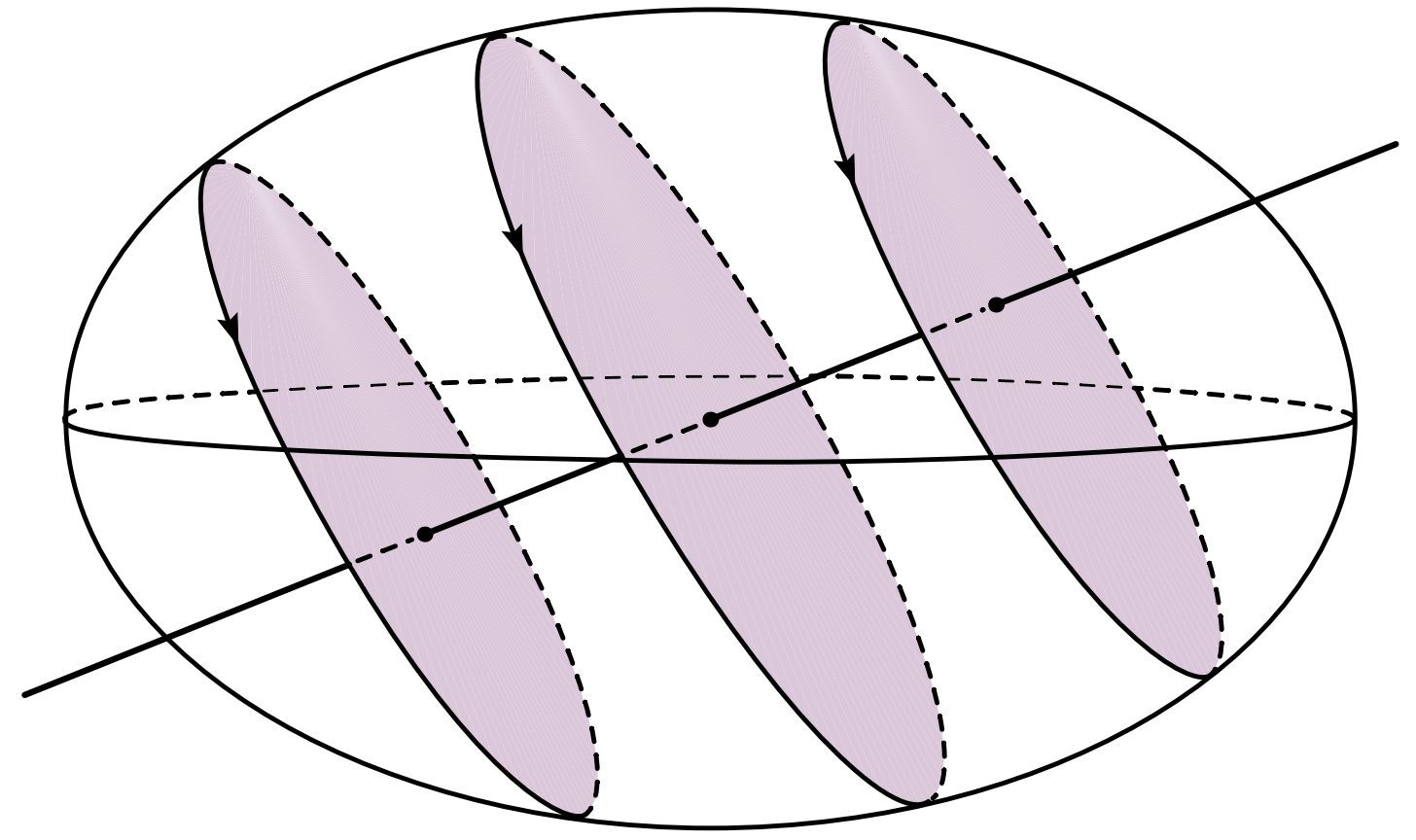

Figure 4.1: Sketch of the Poincaré flow. Three streamlines are shown together with the normal to the planes to which streamlines are confined.

$$
\tan ^{-1} \frac{2|\boldsymbol{\Omega} \times \hat{\boldsymbol{z}}|}{\eta+2(1+\eta) \boldsymbol{\Omega} \cdot \hat{\boldsymbol{z}}}
$$

with respect to the container's equator.

In a new coordinate system formed by a rotation of the $x$ - $z$ plane about the $y$-axis through the angle:

$$
\tan ^{-1} \frac{2 \hat{\boldsymbol{y}} \cdot \boldsymbol{\Omega} \times \hat{\boldsymbol{z}}}{\eta+2(1+\eta) \boldsymbol{\Omega} \cdot \hat{\boldsymbol{z}}}
$$

the velocity is:

$$
\boldsymbol{u}=\frac{1}{2 \sqrt{1+\mu^{2}}}\left[\begin{array}{ccc}
0 & -\left[2+(2+\eta) \mu^{2}\right]+\eta \mu^{2} & 0 \\
{\left[2+(2+\eta) \mu^{2}\right]+\eta \mu^{2}} & 0 & -2 \eta \mu \\
0 & 0 & 0
\end{array}\right] \boldsymbol{x}
$$

with:

$$
\mu=\frac{2 \Omega_{x}}{\eta+2(1+\eta) \Omega_{z}}
$$


The resultant streamlines are ellipses with sheared centres:

$$
\left(x-\frac{2 \varepsilon}{1+\beta} z\right)^{2}+\left(\frac{1-\beta}{1+\beta}\right) y^{2}=\text { const. }
$$

where:

$$
\varepsilon=\frac{\eta \mu}{2+(2+\eta) \mu^{2}} \quad \text { and } \quad \beta=\frac{\eta \mu^{2}}{2+(2+\eta) \mu^{2}}
$$

$\varepsilon$ measures the shearing of the streamlines, whereas $\beta$ measures their elliptical distortion.

The relative magnitude of these two effects depends on the precessional vector $\Omega$ via:

$$
\frac{\beta}{\varepsilon}=\mu=\frac{2 \Omega_{x}}{\eta+2(1+\eta) \Omega_{z}}
$$

At the Earth's parameters:

$$
|\boldsymbol{\Omega}| \simeq 1.06 \times 10^{-7}, \quad \alpha \simeq 23.5^{\circ}, \quad e \simeq 2.45 \times 10^{-3}, \quad \mu \simeq 1.6 \times 10^{-5}
$$

so that the shearing of the streamlines is the dominant effect.

A linear stability analysis can be performed to investigate the evolution of small disturbances upon the basic state, in a frame rotating with this flow, affected by $\varepsilon$ and $\beta$ distortions (Kerswell, 1993).

The equations of motion read:

$$
\begin{aligned}
& \frac{\partial \boldsymbol{u}}{\partial t}+2\left(\begin{array}{r}
-v \\
u \\
0
\end{array}\right)+\nabla p=\varepsilon\left\{e^{i(\varphi+t)} \mathcal{L}_{S}(\boldsymbol{u}, p)+e^{-i(\varphi+t)} \mathcal{L}_{S}^{+}(\boldsymbol{u}, p)\right\}+ \\
& \beta\left\{e^{2 i(\varphi+t)} \mathcal{L}_{E}(\boldsymbol{u}, p)+e^{-2 i(\varphi+t)} \mathcal{L}_{E}^{+}(\boldsymbol{u}, p)\right\} \\
& \nabla \cdot \boldsymbol{u}=0 \\
& \left.\boldsymbol{u} \cdot \hat{\boldsymbol{n}}\right|_{\partial V}=0 \quad \text { with } \quad \partial V: x^{2}+y^{2}+(1+\eta) z^{2}=1
\end{aligned}
$$

where $\mathcal{L}_{S}$ and $\mathcal{L}_{E}$ are the shearing and elliptical operators, respectively, and + indicates complex conjugate (see [30], pag.116).

When the precession is switched off (setting to zero the right-hand side of (4.6)), the solutions of the above system are neutral oscillations called Poincaré modes (see section 1.1). The terms on the right-hand side of (4.6) represent the leading order consequences of switching precession on. They act as coupling terms which allow two Poincaré modes to interact.

In particular, the operators $\mathcal{L}_{S}$ and $\mathcal{L}_{S}^{+}$resonantly couple two Poincaré modes when their azimuthal wavenumbers and frequencies differ by 1 ('shearing' instability), while the 
operators $\mathcal{L}_{E}$ and $\mathcal{L}_{E}^{+}$couple Poincaré modes with azimuthal wavenumbers and frequencies differing by 2 ('elliptical' instability). It is noteworthy to underline that when $\beta=0$ the elliptical operator does not go to zero, because the shearing operator contributes at second order to it.

In an oblate spheroidal container, a Poincaré mode:

$$
[\boldsymbol{u}(\boldsymbol{x}, t), p(\boldsymbol{x}, t)]=[\boldsymbol{Q}(\boldsymbol{x}), \Phi(\boldsymbol{x})] \cdot e^{i \lambda t}
$$

takes the form:

$$
\boldsymbol{Q}=\left[\begin{array}{cc}
\frac{-i}{4-\lambda^{2}} & \left.\lambda \Phi_{r}+\frac{2 m}{r} \Phi\right) \\
\frac{1}{4-\lambda^{2}}\left(2 \Phi_{r}+\frac{m \lambda}{r} \Phi\right) \\
\frac{i}{\lambda} \Phi_{z}
\end{array}\right] e^{i(m \varphi+\lambda t)}
$$

where subscripts $r$ and $z$ indicate the derivatives respect to these variables and $p=$ $\Phi(r, z) e^{i(m \varphi+\lambda t)}$.

The pressure function $\Phi(r, z)$ was derived by Bryan(1889) as the product of two associated Legendre functions and later expressed in a more useful polynomial representation by Cartan(1922), Kudlick(1966), Aldridge and Toomre (1969):

$$
\Phi_{l^{\prime}, m, k}=r^{|m|} z^{\nu} \prod_{j=1}^{N}\left[\bar{x}_{j}^{2}\left(\bar{x}_{j}^{2}-1\right)+\bar{x}_{j}^{2}\left(\frac{r}{A_{l^{\prime}, m, k}}\right)^{2}+\left(1-\bar{x}_{j}^{2}\right)\left(\frac{z}{B_{l^{\prime}, m, k}}\right)^{2}\right]
$$

(here the normalization constants have been skipped), with:

$$
\begin{aligned}
A_{l^{\prime}, m, k}^{2}= & \frac{(1-e)^{2}+\left[1-(1-e)^{2}\right]\left(1-\lambda^{2} / 4\right)}{1-\lambda^{2} / 4} \\
B_{l^{\prime}, m, k}^{2}= & \frac{(1-e)^{2}+\left[1-(1-e)^{2}\right]\left(1-\lambda^{2} / 4\right)}{\lambda^{2} / 4} \\
& \nu=\left\{\begin{array}{lll}
0, & \text { if } l^{\prime}-|m| \text { is even } \\
1, & \text { if } l^{\prime}-|m| \text { is odd }
\end{array}\right.
\end{aligned}
$$

and $\bar{x}_{j}^{2}$ are the $N=\frac{1}{2}\left(l^{\prime}-|m|-\nu\right)$ distinct squared zeros of the associated Legendre polynomial $P_{l^{\prime}}^{|m|}(x)$ in the open interval $(0,1)$.

The eigenvalues $\lambda$ are determined from the no-normal velocity boundary condition which reduces to:

$$
\begin{aligned}
& \left(1-x^{2}\right) \frac{d P_{l^{\prime}}^{|m|}}{d x}(x)=m\left(\frac{1+\eta x^{2}}{1+\eta}\right)^{1 / 2} P_{l^{\prime}}^{|m|}(x) \\
& \text { at } \quad x=\frac{\lambda / 2}{\left[1+\eta\left(1-\frac{\lambda^{2}}{4}\right)\right]^{1 / 2}}
\end{aligned}
$$


An alternative form of the eigenvalue relation (4.9) can be derived using (4.8) so that the algebraic problem for the eigenfrequency $\lambda$ is:

$$
\nu+2 \sum_{j=1}^{N} \frac{\lambda^{2}(1-e)^{2}}{\lambda^{2}(1-e)^{2}-\bar{x}_{j}^{2}\left\{4-\lambda^{2}\left[1-(1-e)^{2}\right]\right\}}=\frac{m(1-e)^{2} \lambda}{(2-\lambda)}
$$

when $m \geq 0$. For negative $m$, the relationship: $\lambda_{l^{\prime},-m, k}=-\lambda_{l^{\prime}, m, k}$ holds.

The equation (4.11) shows that, in general, there will be several eigenvalues corresponding to given indices $\left(l^{\prime}, m\right)$. Therefore, the subscript $k$ used above labels the particular eigenfrequency and has a maximum value given by:

$$
k_{\text {max }}\left(l^{\prime}, m\right)= \begin{cases}\left(l^{\prime}-1\right), & \text { if } m=0 \\ \left(l^{\prime}-|m|\right), & \text { if } m \neq 0\end{cases}
$$

In particular: $k_{\text {max }}\left(l^{\prime}, \pm l^{\prime}\right)=0$, i.e. there are no Poincaré modes with $l^{\prime}=|m|$.

In order to compute numerically the eigenfrequency $\lambda_{l^{\prime}, m, k}$, equation (4.9) has been used, instead of (4.11), in that the latter presents some singularities (when the denominator vanishes) which lead to spurious roots!

Applying the following recurrence relation for the Legendre polynomials:

$$
\left(1-x^{2}\right) \frac{d P_{l^{\prime}}^{|m|}}{d x}(x)=-l^{\prime} x P_{l^{\prime}}^{|m|}(x)+\left(l^{\prime}+m\right) P_{l^{\prime}-1}^{|m|}(x)
$$

to the equation (4.9), one obtains:

$$
\left[m\left(\frac{1+\eta x^{2}}{1+\eta}\right)^{1 / 2}+l^{\prime} x\right] P_{l^{\prime}}^{|m|}(x)-\left(l^{\prime}+m\right) P_{l^{\prime}-1}^{|m|}(x)=0
$$

Once the zeros of (4.12) are computed numerically, the eigenfrequency $\lambda$ can be evaluated from the relation (4.10).

As it has already been mentioned, resonant coupling between two Poincaré modes is essentially that of a triad interaction, where the 'third wave' represents the distortion of the basic state. When the shear of the streamlines is the dominant effect, the 'third wave' has frequency 1 and azimuthal wavenumber 1, with respect to the rotating frame, leading to the following resonant conditions:

$$
\begin{aligned}
\lambda_{b} & =\lambda_{a}+1 & \text { 'shearing' instability } \\
m_{b} & =m_{a}+1 &
\end{aligned}
$$

with $a$ and $b$ labeling two different Poincaré modes. If the elliptical distortion of the streamlines plays a dominant role, the 'third wave' of the basic state possesses a frequency of 2 and azimuthal wavenumber 2 , leading to the following resonant conditions:

$$
\begin{array}{ll}
\lambda_{b} & =\lambda_{a}+2 \\
m_{b} & =m_{a}+2
\end{array} \quad \text { 'elliptical' instability }
$$


Moreover, a further resonance condition to be satisfied if shearing or elliptical instabilities are excited is:

$$
l_{b}^{\prime}=l_{a}^{\prime}
$$

(where $l^{\prime}$ is the modal degree).

A Poincaré mode of index $l^{\prime}$ cannot reach other modes of different indices through the operators $\mathcal{L}_{S}$ or $\mathcal{L}_{S}^{+}, \mathcal{L}_{E}$ or $\mathcal{L}_{E}^{+}$(see [30] for a proof).

Figures 4.2 and 4.3 show, for every fixed $l^{\prime}$, all the existing couplings between two Poincaré modes satisfying the shearing instability resonant conditions (fig.4.2) and the elliptical instability resonant conditions (fig.4.3) as function of the elliptical geometry $e$. The inclusion of viscous effects causes a change in the above resonant conditions in that a detuning term $\Delta$ (defined as the inverse of the viscous decay time and consequently as the spectral width due to frequency displacements) has to be added on the right hand side of (4.13) and (4.14). In viscous bounded fluids, theory predicts that $\Delta$ can be estimated to be approximately proportional to $\sqrt{E}$. Figures 4.4 and 4.5 illustrate the effect of taking into account an Ekman number ranging around $10^{-4}-5 \times 10^{-5}$ in the shearing and elliptical instability resonant conditions, respectively. In this case, (4.13) and (4.14) need to be only 'approximately' satisfied within an error given by: $\Delta \propto \sqrt{5 \times 10^{-5}}-\sqrt{10^{-4}} \simeq 0.7 \times 10^{-2}-10^{-2}$. A comparison with the analogous pictures corresponding to the pure inviscid case (fig.4.2 and fig.4.3) shows that the effect of viscosity is to smear out the precise resonant geometry at which (4.13) and (4.14) are satisfied, including a finite-size neighbourhood.

\subsection{Experimental evidence}

Several laboratory experiments have been performed over the years in order to study the strong three-dimensional instability arising when inertial waves are driven to finite amplitude.

McEwan(1970) considered forced inertial oscillations which appear in an axially rotating circular cylinder of fluid perturbed by precessing the lid. He first used the term 'resonant collapse' in order to describe the phenomenon in which large amplitude resonant oscillations, after persisting in an apparently laminar form, degenerate suddenly into a disordered state from which they do not recover. His two most important conclusions were that linear inviscid theory accurately predicts the geometrical conditions and the frequencies at which low-order inertial wave modes resonate, but that this same analysis will ultimately fail in describing the evolution of the oscillations. McEwan observed three phases in the collapse:

(a) unsteadiness, detected first only in some localized regions of the container, grew rapidly and extended progressively through the whole cylinder;

(b) the pattern became completely disordered; 

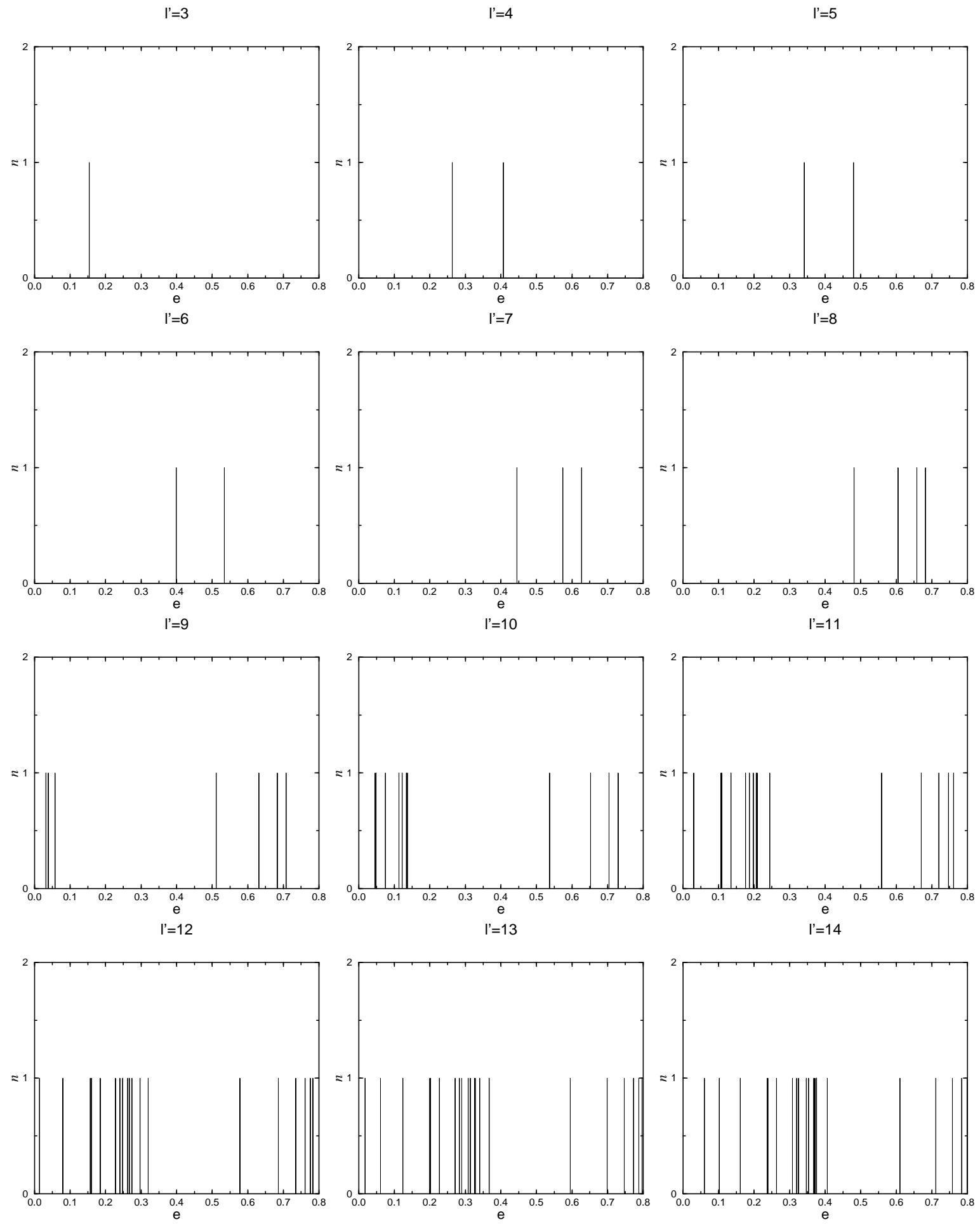

Figure 4.2: Shearing couplings between two Poincaré modes in the pure inviscid case. The vertical lines indicate the ellipticity at which resonances occur and $n$ stands for the number of couplings existing at every fixed $l^{\prime}$. 

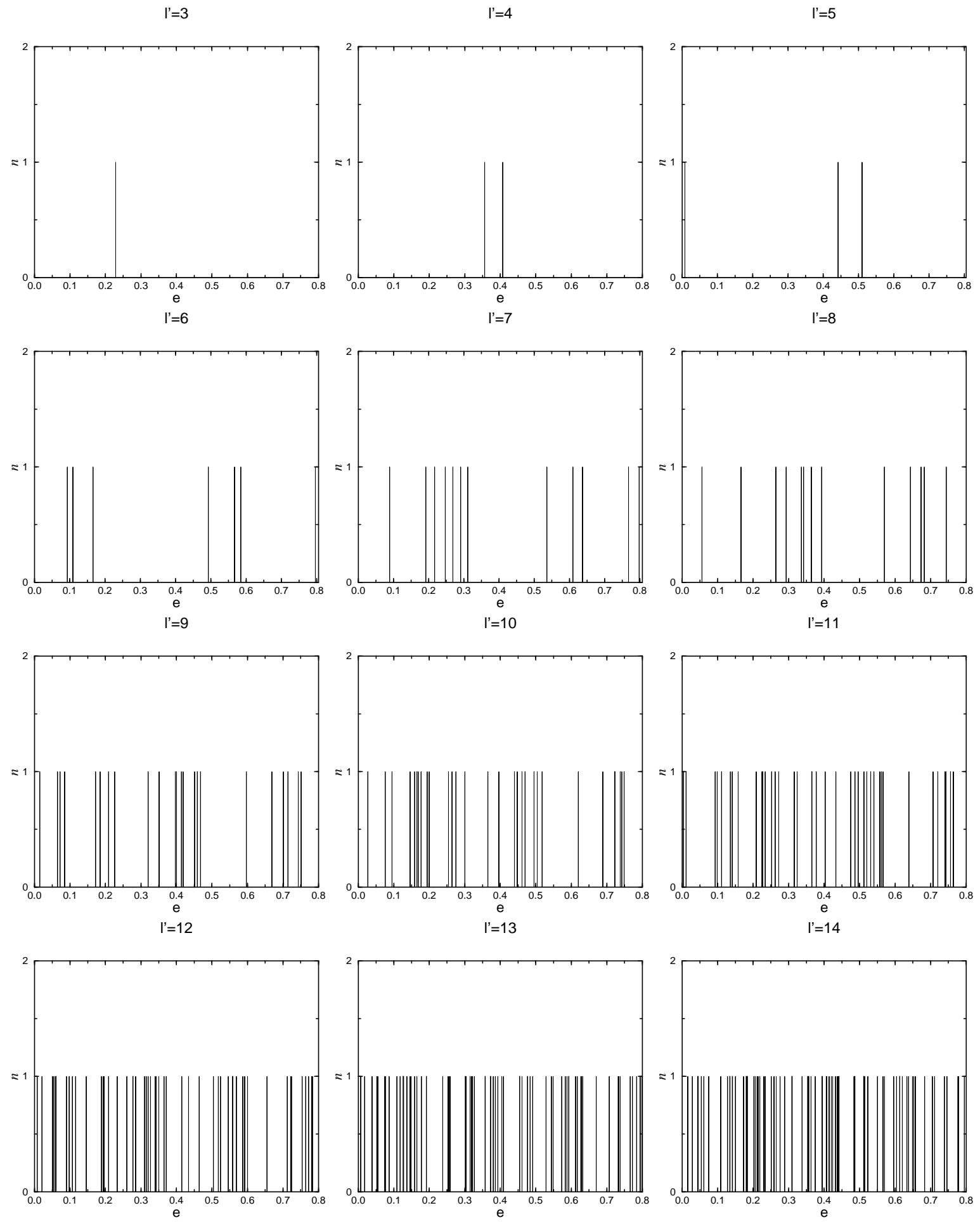

Figure 4.3: Elliptical couplings between two Poincaré modes in the pure inviscid case. The vertical lines indicate the ellipticity at which resonances occur and $n$ stands for the number of couplings existing at every fixed $l^{\prime}$. 

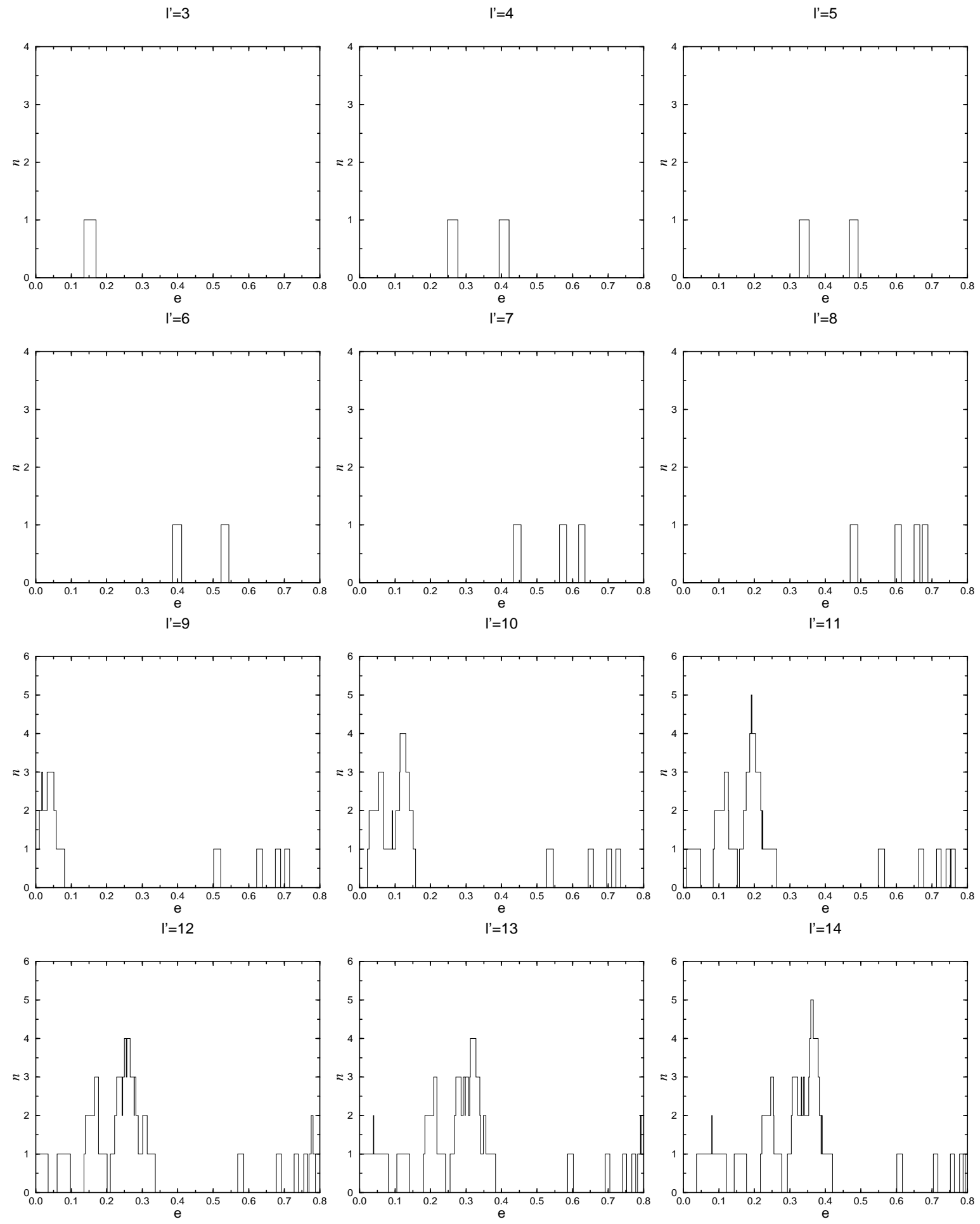

Figure 4.4: Shearing couplings between two Poincaré modes in the viscous case corresponding approximately to an Ekman number ranging from $10^{-4}$ to $5 \times 10^{-5}$. $n$ stands for the number of couplings existing at every fixed $l^{\prime}$ and $e$ is the ellipticity of the container. 

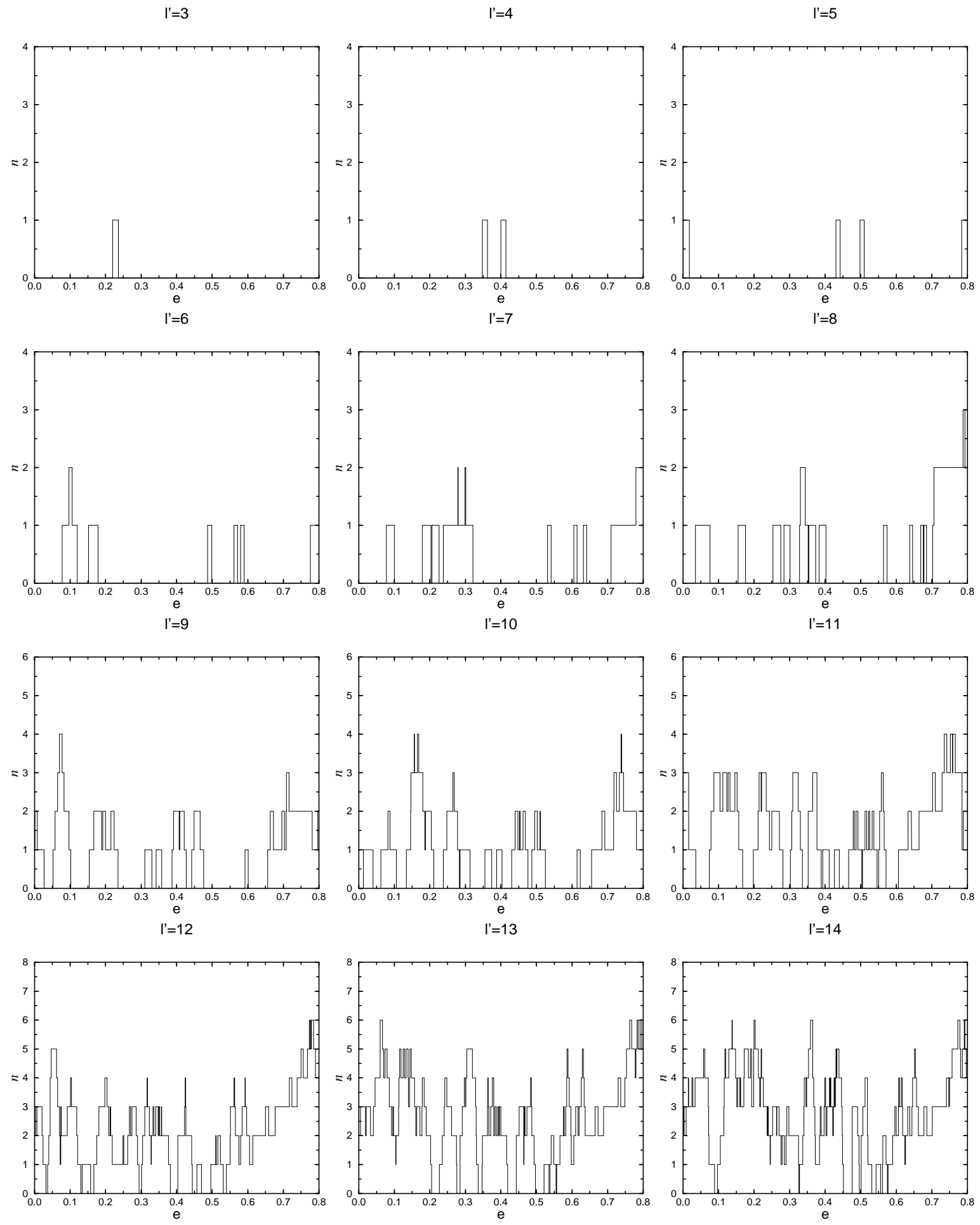

Figure 4.5: Elliptical couplings between two Poincaré modes in the viscous case corresponding approximately to an Ekman number ranging from $10^{-4}$ to $5 \times 10^{-5}$. $n$ stands for the number of couplings existing at every fixed $l^{\prime}$ and $e$ is the ellipticity of the container. 
(c) at higher rotation speeds the disorder appeared to degenerate to a finer scale, fully turbulent.

Weaker excitation or low rotation rate made the collapse less dramatic. After collapse a steady pattern was never fully regained, although the agitation waxed and waned.

Manasseh(1992) reported similar results in a rotating completely filled right circular cylinder perturbed by precessing the whole. In his work there was a confirmation of the two main conclusions presented by McEwan and also of the repeatability of the violent-collapse process. Both authors speculated that the 'resonant collapse' was caused by nonlinear interactions of triads of waves:" the quadratic nonlinear terms combine two modes to force a third; energy supplied to a low-order 'primary' mode can flow to a pair of 'parasitic' secondary modes and so on in a cascade to turbulence."([46], pag.294).

Similar phenomena have been observed in the context of studies of elliptical flow instabilities.

Gledzer et al.(1975) were the first to study experimentally the instability due to the elliptical deformation of the streamlines. They used a rigid water-filled cylinder with elliptical cross-section whose rotation was abruptly stopped after solid body rotation was reached. The fluid was thus forced to follow elliptical streamlines. The main inconvenience of their experiment was the short time during which the instability was observed and the development of a competitive centrifugal instability growing near the wall.

This defect was avoided by Malkus(1989) who used a cylinder with flexible sidewalls deformed by a pair of rollers fixed at opposite sides in the laboratory frame of reference. The simplest 'subharmonic' form of an elliptical instability, where the coupled inertial waves are a complex conjugate pair, has been excited in the experiment. As consequence, the most striking feature of the total velocity field was the wavy rotation axis which has been clearly seen in other laboratory experiments. The observed character of the instability was intermittent: the unstable waves grew, broke down, decayed and then grew again. The most disconcerting aspect of Malkus' experiment was the invariable collapse of the unstable inertial waves despite every effort to isolate such weakly nonlinear saturated states.

A detailed numerical study of this elliptical instability observed in Malkus' experiment has been carried out by Mason \& Kerswell(1999).

Another series of experiments were performed by Malkus with a rigid cylinder placed in the center of the tidally distorted outer cylinder. Various radii were used for the inner cylinder. The annular gap in this experiment prevented the possibility of a growing spinover mode $(m= \pm 1)$ and the instability was a high zonal wavenumber disturbance related to the gap width.

Noteworthy, for the implications on the Earth's fluid core, are the experiments carried out by Aldridge et al.(1997). They investigated the elliptical instability of a rotating fluid, contained in a thick spherical shell, excited by a tide-like perturbation of the flexible inner boundary.

More recently, C.Eloy et al.(2000), using a rotating deformable cylinder on which two or three rollers were applied, have extended the previous observations in elliptic geom- 
etry including triangular deformations of the cylinder. A cycle of instability-disorderrelaminarization was detected, as already reported by Malkus(1989). However, contrary to what this latter author observed, for Reynolds numbers sufficiently close to threshold, the unstable mode grows and reaches a saturation state which remains forever. They reported also some evidence about a saturated secondary instability.

Some authors ([46], [30]) have explained Malkus' experiments in precessing spheroidal cavity (1968), described in detail in section 1.4, as a clear evidence of the elliptical instability mechanism although Malkus suggested that the axisymmetric internal cylindrical shear layers should be considered the source of the observed instability.

Indeed, one of the main aims of this research work is to provide an unambiguous interpretation of these series of experiments through a detailed understanding of the characterizing features of both viscous and inertial instabilities.

\subsection{Numerical methods}

In chapter 2 , it has been evidenced that the range of parameters accessible numerically, adopting realistic no-slip boundary conditions, is limited mainly by the onset of the boundary layer instability accompanied by unresolvably small scales. On the other hand, theory and experiments revealed a fascinating energetic instability mechanism, involving coupled Poincaré modes, which leads eventually to a violent turbulent motion.

In order to gain some evidence for the inertial instability, which is a completely inviscid mechanism, within the precessing ellipsoidal cavity considered in this context, one needs to get rid of the Ekman boundary layers where the viscosity damping is stronger. The most elegant way to do so is to use stress free boundary conditions. In that case, the Ekman layers disappear in favor of much weaker boundary layers which exert virtually no influence on the interior flow. The laminar flow is then practically identical to Poincaré's solution.

Using, as in section 2.2, two coordinate systems: the original one, in which the boundaries are ellipsoids of revolution (described by symbols with tildes) and the computational one, in which the boundaries are spherical, a normal to the fluid ellipsoidal boundary is given by:

$$
\tilde{\boldsymbol{n}}=2\left(\tilde{x}, \tilde{y}, \tilde{z} /(1-e)^{2}\right)
$$

The condition that no fluid traverses this boundary ('inviscid boundary condition') becomes:

$$
\tilde{\boldsymbol{u}} \cdot \tilde{\boldsymbol{n}}=2\left(u_{x} x+u_{y} y+u_{z} z\right)=2(\boldsymbol{u} \cdot \boldsymbol{r})=0
$$

In terms of the poloidal and toroidal scalars, this means that $V_{l}^{m}(r)$ must be zero at the boundaries, which is a condition already implemented for the previous no-slip boundaries.

The stress at the boundaries is given (to within a factor of 2) by: 


$$
\boldsymbol{T} \tilde{\boldsymbol{n}}=\boldsymbol{t}_{s}+2 e \frac{2-e}{(1-e)^{2}} z \frac{\partial}{\partial z} \boldsymbol{u}+2 \hat{\boldsymbol{z}}\left(\frac{e}{(1-e)} \boldsymbol{r} \frac{\partial}{\partial z} \boldsymbol{u}-e(\boldsymbol{r} \cdot \nabla) u_{z}-e^{2} \frac{2-e}{(1-e)^{2}} z \frac{\partial}{\partial z} u_{z}\right)
$$

where:

$$
T_{i j}=\frac{\partial}{\partial \tilde{x}_{i}} \tilde{u}_{j}+\frac{\partial}{\partial \tilde{x}_{j}} \tilde{u}_{i}
$$

and $\boldsymbol{t}_{\boldsymbol{s}}$, expressed in vectorial form as:

$$
t_{s, i}=\left(\frac{\partial}{\partial x_{i}} u_{j}+\frac{\partial}{\partial x_{j}} u_{i}\right) n_{j} ; \quad \boldsymbol{n}=2(x, y, z)
$$

is the stress exerted on a spherical surface whose normal is $\boldsymbol{n}$.

At the boundaries (where $u_{r}=0$ ) $\boldsymbol{t}_{s}$ reads in polar coordinates:

$$
\begin{aligned}
\hat{\boldsymbol{r}} \boldsymbol{t}_{s} & =4 r \frac{\partial}{\partial r} u_{r} \\
\hat{\boldsymbol{\vartheta}} \boldsymbol{t}_{s} & =2 r^{2} \frac{\partial}{\partial r}\left(\frac{u_{\vartheta}}{r}\right) \\
\hat{\boldsymbol{\varphi}} \boldsymbol{t}_{s} & =2 r^{2} \frac{\partial}{\partial r}\left(\frac{u_{\varphi}}{r}\right)
\end{aligned}
$$

The tangential directions to the fluid ellipsoidal surface are given by $\hat{\varphi}$ and $\hat{\tau}$ with:

$$
\hat{\boldsymbol{\tau}}=2 r\left[\frac{e}{(1-e)} \sin \vartheta \cos \vartheta \hat{\boldsymbol{r}}+\left(1+\frac{e}{(1-e)} \cos ^{2} \vartheta\right) \hat{\boldsymbol{\vartheta}}\right]
$$

The first boundary condition, $\hat{\boldsymbol{\varphi}} \boldsymbol{T} \tilde{\boldsymbol{n}}=0$, therefore becomes:

$$
-\frac{u_{\varphi}}{r}+\left[1+\frac{e(2-e)}{(1-e)^{2}} \cos ^{2} \vartheta\right] \frac{\partial u_{\varphi}}{\partial r}-\frac{e(2-e)}{(1-e)^{2}} \frac{\sin \vartheta \cos \vartheta}{r} \frac{\partial u_{\varphi}}{\partial \vartheta}=0
$$

A similar but more involved expression is deduced from the second boundary condition, $\hat{\boldsymbol{\tau}} \boldsymbol{T} \tilde{\boldsymbol{n}}=0$ :

$$
\begin{aligned}
& \frac{1}{(1-e)}\left[-1+\frac{e^{2}(2-e)^{2}}{(1-e)^{2}} \sin ^{2} \vartheta \cos ^{2} \vartheta\right] u_{\vartheta}+ \\
& r\left[1+\frac{e}{(1-e)} \cos ^{2} \vartheta-e \sin ^{2} \vartheta\right]\left[1+\frac{e(2-e)}{(1-e)^{2}} \cos ^{2} \vartheta\right] \frac{\partial u_{\vartheta}}{\partial r}+ \\
& r e \frac{(2-e)}{(1-e)} \sin \vartheta \cos \vartheta\left[1+\frac{e(2-e)}{(1-e)^{2}} \cos ^{2} \vartheta\right] \frac{\partial u_{r}}{\partial r}- \\
& \frac{e(2-e)}{(1-e)^{2}}\left[1+\frac{e}{(1-e)} \cos ^{2} \vartheta-e \sin ^{2} \vartheta\right] \sin \vartheta \cos \vartheta \frac{\partial u_{\vartheta}}{\partial \vartheta}=0
\end{aligned}
$$




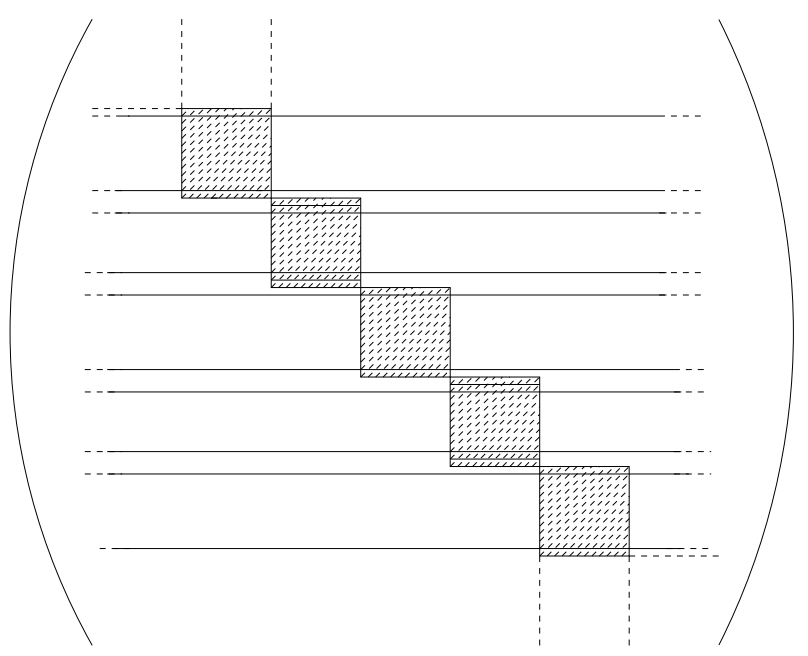

Figure 4.6: Structure of the matrix $\boldsymbol{M}_{1}$. The diagonal contains blocks of dimension $N_{r} \times N_{r}$. Rows corresponding to the boundary conditions given by (4.16) and (4.17), within each block, extend beyond the diagonal band of blocks.

The time step procedure is similar to the one for no-slip boundaries (section 2.2), except that the boundary conditions now couple the poloidal and toroidal scalars and different $l$ (see fig.4.6).

The free-slip boundary conditions are included in the matrix $\boldsymbol{M}_{1}$ appearing in the discretization of the implicit part of the time step [section 2.2.2, equation (2.44)]. The two inner and outermost collocation points of the dynamic equation for the poloidal field [equ.(2.34)] are replaced with (4.15) and (4.17), whereas the equation for the toroidal scalar (2.35) on the boundaries of the computational domain is replaced with (4.16). This appears a natural choice because the discretization appropriate for a spherical container is then automatically recovered in the limit of zero ellipticity. $\boldsymbol{M}_{1}$ thus is a sparse matrix since, apart from the boundary conditions, it consists of small blocks of size $N_{r} \times N_{r}$. It is therefore advantageous to invert $\boldsymbol{M}_{1}$ with the help of the Woodbury formula, which is the block-matrix version of the Sherman-Morrison formula.

Writing the change in the matrix $\boldsymbol{M}_{1}$, due to the free-slip boundary conditions, in the form:

$$
\boldsymbol{M}_{1} \Longrightarrow\left(\boldsymbol{M}_{1}+\boldsymbol{U} \cdot \boldsymbol{V}^{T}\right)
$$

( $T$ indicates transpose), where $\boldsymbol{V}^{T}$ is a matrix containing the boundary conditions and $\boldsymbol{U}$ a matrix filled only with ones and zeros, whose dimensions are much smaller than $\boldsymbol{M}_{1}$, then the corrected inverse matrix can be calculated by:

$$
\left(\boldsymbol{M}_{1}+\boldsymbol{U} \cdot \boldsymbol{V}^{T}\right)^{-1}=\boldsymbol{M}_{1}^{-1}-\left[\boldsymbol{M}_{1}^{-1} \cdot \boldsymbol{U} \cdot\left(1+\boldsymbol{V}^{T} \cdot \boldsymbol{M}_{1}^{-1} \cdot \boldsymbol{U}\right)^{-1} \cdot \boldsymbol{V}^{T} \cdot \boldsymbol{M}_{1}^{-1}\right]
$$


The matrices $\boldsymbol{V}^{T}$ and $\boldsymbol{U}$ are computed during the initialization and stored for later use. The Woodbury formula is applied within every Jacobi type iteration.

The operation load per time step is of course increased compared with the no-slip version because of the couplings introduced by the boundary conditions. However, at equal resolution, one can reach much more extreme parameters, especially regarding the Ekman number. At a typical resolution $\left(N_{r}=129, L=128, M=64\right)$ the CPU time per time-step has increased by a factor 3.7. Because of the more complicated structure of $\boldsymbol{M}_{1}$, memory requirements have increased by a factor 13.8 .

\subsection{Instabilities}

With free-slip boundaries, the laminar flow is practically identical to Poincaré's solution in that the weak Ekman layers generated do not affect the interior motions. This enabled us to test the numerical code at small precession rates when stable flows are obtained. Indeed, Poincaré's solution does not satisfy stress free boundary conditions so that an internal circulation is expected to arise. This secondary circulation is however negligible and a good agreement between the simulated laminar flows and the analytical results given by Poincaré (section 2.1.1) has been found. A further and more robust test of the numerical code (for the purposes of this research) has been realized choosing a set of parameters for which it is possible to predict an inertial instability on theoretical grounds.

Analytically, some exact solutions of the linearized Euler equations, describing the evolution of a disturbance upon Poincaré's basic state $\boldsymbol{U}$, can be constructed, which are valid for arbitrary precession rates (Kerswell, 1993).

The linearized disturbance equations in the precessing frame (where the $z$-axis is chosen to coincide with the spheroidal axis of symmetry) are:

$$
\begin{gathered}
\frac{\partial \boldsymbol{u}}{\partial t}+2 \boldsymbol{\Omega} \times \boldsymbol{u}+(\boldsymbol{U} \cdot \nabla) \boldsymbol{u}+(\boldsymbol{u} \cdot \nabla) \boldsymbol{U}+\nabla p=0 \\
\nabla \cdot \boldsymbol{u}=0,\left.\quad \boldsymbol{u} \cdot \hat{\boldsymbol{n}}\right|_{\partial V}=0 \quad \partial V: x^{2}+y^{2}+(1+\eta) z^{2}=1
\end{gathered}
$$

where, assuming an angle of $90^{\circ}$ between the container and precession axes, such that $\boldsymbol{\Omega}=\Omega \hat{\boldsymbol{x}}, \boldsymbol{U}$ is written as follows:

$$
\boldsymbol{U}=\left[\begin{array}{ccc}
0 & -1 & 0 \\
1 & 0 & -(1+\eta) \mu \\
0 & \mu & 0
\end{array}\right] \boldsymbol{x}
$$

and $\mu=2 \Omega / \eta$.

The eigensolutions of the above system are:

$$
\boldsymbol{u}(\boldsymbol{x}, t)=\tilde{\boldsymbol{Q}}(\boldsymbol{x}) e^{i \Lambda t}
$$

where $\Lambda$ is the eigenfrequency in the precessing frame. For $\boldsymbol{\Omega}=0$, the eigensolutions are the Poincaré modes: 


$$
\boldsymbol{Q}_{l^{\prime}, m, k}(\boldsymbol{x}) e^{i \Lambda t}
$$

which belong to orthogonal vector spaces $\mathcal{V}_{l^{\prime}}$ :

$$
\mathcal{V}_{l^{\prime}}=<Q_{l^{\prime}, m, k}, \quad-l^{\prime} \leq m \leq l^{\prime}, \quad k=1, \cdots, k_{\text {max }}>
$$

Kerswell proved that, when the precession rate $|\boldsymbol{\Omega}|$ is increased from zero, the disturbed eigensolutions $\tilde{Q}_{l^{\prime}, m, k}$ are superpositions of the Poincaré basic modes $\boldsymbol{Q}_{l^{\prime}, m, k}$ belonging to one and only one vector space $\mathcal{V}_{l^{\prime}}$ which is invariant as $\Omega$ changes:

$$
\begin{aligned}
\mathcal{V}_{l^{\prime}}= & <\tilde{Q}_{l^{\prime}, m, k}, \quad-l^{\prime} \leq m \leq l^{\prime}, \quad k=1, \cdots, k_{\max }>= \\
& <Q_{l^{\prime}, m, k}, \quad-l^{\prime} \leq m \leq l^{\prime}, \quad k=1, \cdots, k_{\max }>
\end{aligned}
$$

Since the Poincaré modes are basis vectors for every $\mathcal{V}_{l^{\prime}}$, by definition, the eigensolutions are:

$$
\boldsymbol{u}(\boldsymbol{x}, t)=\sum_{m, k} \alpha_{m, k} \boldsymbol{Q}_{l^{\prime}, m, k}(\boldsymbol{x}) e^{\sigma t}
$$

Considering the simplest vector space $\mathcal{V}_{3}$, which represents the space of all incompressible, quadratic velocities, equation (4.20) becomes an eigenvalue problem for the growth rate $\sigma$ depending on two parameters: the geometry $\eta$ and the precession rate $\Omega$. A shearing instability is expected to occur between $\boldsymbol{Q}_{3,1,2}$ and $\boldsymbol{Q}_{3,2,1}$ at $\eta=0.3955(e=0.15)$ with growth rate $\sigma=0.3531 \varepsilon$ [see figure 4.2]. The precession rate at which such kind of coupling is excited, according to the analysis reported above, is about $|\boldsymbol{\Omega}| \simeq 0.1$.

In conformity with this theoretical scenario, a test-run has been performed corresponding to the following parameters:

$$
\alpha=90^{\circ}, \quad \Omega=-0.1, \quad e=0.15 \text { and } E=10^{-4} .
$$

Since the inertial instability is an entirely inviscid mechanism, the role played by the Ekman number $E$ has to be regarded with particular care. Indeed, even if there are many opportunities for resonances to occur as figures 4.2 and 4.3 show, the actual growth of two coupled Poincaré modes depends crucially on the fluid viscosity. Moreover, the presence of viscosity can be seen as a random factor which acts stabilizing for some triads and destabilizing for others through producing viscous frequency shifts [see figures 4.4, 4.5].

With free-slip boundary conditions, the viscous decay rate can be approximately estimated as:

$$
\nu=s \cdot l(l+1) E
$$

where $s$ is a coefficient of $O(1)$, and $l$ is the modal index appearing in decompositions as in (2.33). In the Navier-Stokes equations, the angular part of the diffusion term $E \nabla^{2} \boldsymbol{u}$, after a decomposition of the velocity field in spherical harmonics, assumes the form: 


$$
\nabla^{2} Y_{l}^{m}(\vartheta, \varphi)=-\frac{L_{2}}{r^{2}} Y_{l}^{m}(\vartheta, \varphi)
$$

with:

$$
L_{2} Y_{l}^{m}(\vartheta, \varphi)=l(l+1) Y_{l}^{m}(\vartheta, \varphi)
$$

This is precisely the correction factor which has been taken into account in equation (4.21). The difficulty which arises in order to connect directly the modal degree $l$ with the $l^{\prime}$-index introduced in the previous discussion on Poincaré modes is twofold:

(a) the equation of motion (2.28) couples different $l$ so that the $l-m$ components of the velocity field at every time contain contributions from $l-1, l+1$ (due to the Coriolis term) as well as from $l-2, l+2$ (due to the linear terms appearing in (2.30));

(b) the modal dependence of Poincaré modes is actually described by two indices $l^{\prime}$ and $k$ (see eq.(4.8)).

Only on the surface of the spheroid, all the functions $\Phi_{l^{\prime}, m, k}$ (eq.(4.8)) reduce to the same zonal harmonic $([25])$ and the inertial modes lose consequently their dependence on $k$. In this simplified context $l^{\prime}$ can be regarded as the modal degree to be substituted in (4.21) in order to obtain an average estimate (due to the point (a)) of the viscous damping.

Once the viscous decay rate is evaluated, formulae (4.4) and (4.5) give an idea of the relative strength of the two mechanisms of instability: if the shearing dominates $(\mu<1)$ the typical growth rate for shear instability is:

$$
\sigma=\bar{\sigma}_{\varepsilon} \cdot \varepsilon
$$

vice versa, if the elliptical distortion of the streamlines prevails $(\mu>1)$ the growth rate for elliptical instability is:

$$
\sigma=\bar{\sigma}_{\beta} \cdot \beta
$$

$\bar{\sigma}_{\varepsilon}$ and $\bar{\sigma}_{\beta}$ are two coefficients which can be calculated analytically through formulae as in (4.20), or simply set equal to the upper bounds estimated by an unbounded analysis of fluids whose streamlines are elliptically distorted or sheared.

In this latter case:

$$
\bar{\sigma}_{\beta}^{u p p e r} \simeq 0.5625
$$

(Waleffe, 1989)

$$
\bar{\sigma}_{\varepsilon}^{u p p e r} \simeq 0.6052
$$

(Kerswell, 1993).

In the test-run introduced above, the viscous decay rate can be computed as follows: 


$$
\nu \simeq s \times 3 \times 4 \times 10^{-4} \simeq s \times 1.2 \times 10^{-3}
$$

using $l^{\prime}=3$ as predicted by the analytical calculations for the simplest coupling.

At the chosen parameters, the shearing strain is the dominant effect, in that from (4.4) and (4.5):

$$
\begin{aligned}
& |\mu| \simeq 0.51 \\
& |\varepsilon| \simeq 7.66 \times 10^{-2} \\
& \beta \simeq 3.87 \times 10^{-2}
\end{aligned}
$$

Therefore the growth rate (estimated analytically) is:

$$
\sigma \simeq 0.3531 \varepsilon \simeq 2.70 \times 10^{-2}
$$

Since the shearing growth rate exceeds the viscous damping rate of at least one order of magnitude, the excitation of shear instability is expected on theoretical grounds. Numerical calculations confirm these inferences. Poincaré's basic state given by (4.1) and (4.2) has been considered as initial condition for all the simulations presented in this section. After a short lag, two modes of azimuthal wavenumbers 1 and 2 have been seen to grow. This instability (as the viscous instability presented in chapter 2) breaks the symmetry of the basic flow and a decomposition of the velocity field in symmetric and antisymmetric components turns out once more to be a useful tool of investigation. The initial linear growth rate of the instability has been evaluated through the numerical computation of the slope of the increasing antisymmetric energy, $E_{a}$ :

$$
\left(2 \sigma^{(n)}\right) \simeq 3.97 \times 10^{-2}
$$

where:

$$
E_{a}=\frac{1}{2} u_{a}^{2} \simeq \frac{1}{2} e^{2 \sigma^{(n)} t} \Longrightarrow \sigma^{(n)}=\frac{\left(2 \sigma^{(n)}\right)}{2} \simeq 1.985 \times 10^{-2}
$$

which has to be compared with the analytical result (4.26). The two values are compatible considering the viscous damping included in the numerical growth rate.

While the picture painted throughout the analytical work has been a linear one, the numerical results presented in the following can be considered a new contribution in understanding the secondary, nonlinear phases of an inertial instability which exist beyond the initial stage of exponential growth.

Assuming that, as for the previous viscous instability, the inertial disturbances have a well defined wavenumber with respect to the fluid axis (that is confirmed a-posteriori by the numerical simulations), a new coordinate system denoted by primes in which the $z^{\prime}$ axis points along $\boldsymbol{\omega}_{F}$ has been introduced. Long before the antisymmetric energy reaches its final saturated state, the two unstable modes $m^{\prime}=1$ and 2 manifest themselves as elongated rolls propagating around the fluid axis. 
Figure 4.7 shows cylindrical cuts in which the $r$ and $z^{\prime}$ components of the antisymmetric velocity field have been drawn.
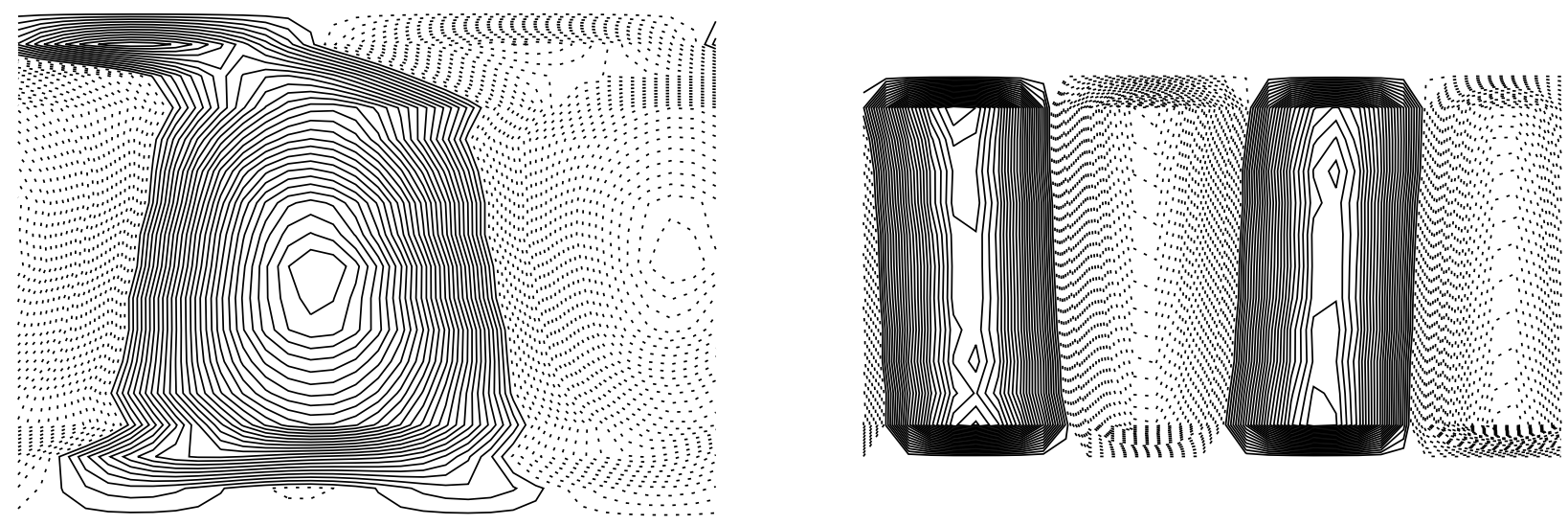

Figure 4.7: $u_{a r}$ (left) and $u_{a z^{\prime}}$ (right) on cylindrical surfaces at distances 0.34 (left) and 0.69 (right) from the $\boldsymbol{\omega}_{F}$-axis for $\eta=0.1, e=0.15, \alpha=90^{\circ}, \Omega=-0.1$ and $E=10^{-4} \cdot \varphi^{\prime}$ runs from $-\pi$ to $\pi$ in going from left to right and $\vartheta^{\prime}$ runs from -80 to 80 (left) and from -60 to 60 (right) in going from bottom to top.

As for viscous instabilities, associated with the odd $m^{\prime}$ one finds vortices symmetric about the equatorial plane of the primed coordinate system (in this particular example with $m^{\prime}=1$, there are only two big vortices of opposite sign), whereas the evenwavenumber vortices are antisymmetric about this plane.

The unstable waves are seen to grow for some time and then to collapse into small-scale disorder.

Figure 4.8 reproduces the shape assumed by the antisymmetric energy in its saturated final state. Regular big-amplitude (one order of magnitude) and long-period ( $T \simeq 200$ units of time) oscillations appear.

This reveals to be a classic example of inertial wave breakdown observed in experiments where inertial waves are driven to finite amplitude (McEwan, 1970; Gledzer, 1975; Malkus, 1989; Manasseh, 1992, 1994, 1996; Aldridge et al. 1997; Eloy et al. 2000).

The mechanism through which an inertial instability develops during its nonlinear phase is better understood looking at figure 4.9 .

Here the total antisymmetric energy and the fraction of $E_{a}$ contained in modes $m^{\prime}>$ 2 (i.e. other than the two modes in which the inertial instability resides) are plotted separately, during the nonlinear oscillating phase.

The unstable coupled inertial modes grow until they saturate and break down into small scales. This cascade of energy from large to small scales is clearly visible in the plot: at every maximum energy is transferred to modes with high wavenumbers which are excited with a small delay in their phase. This disordered state is not maintained by the precessional motion of the spheroidal cavity, but it evolves back by a 'large-scales 


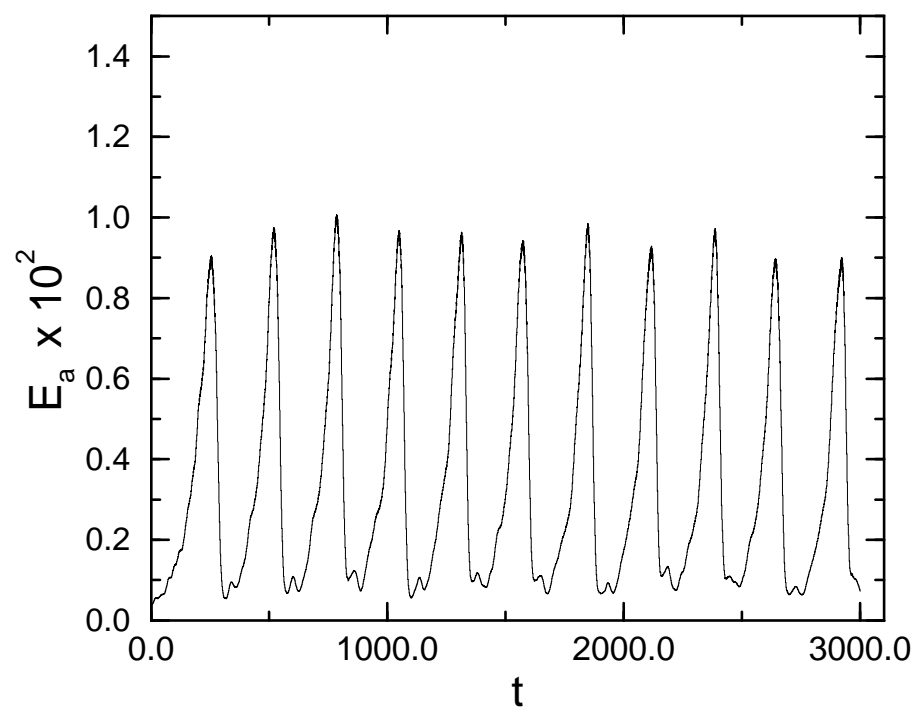

Figure 4.8: Time evolution of the energy $E_{a}$ contained in the velocity components antisymmetric with respect to reflection at the origin for the same parameters as in figure 4.7 .

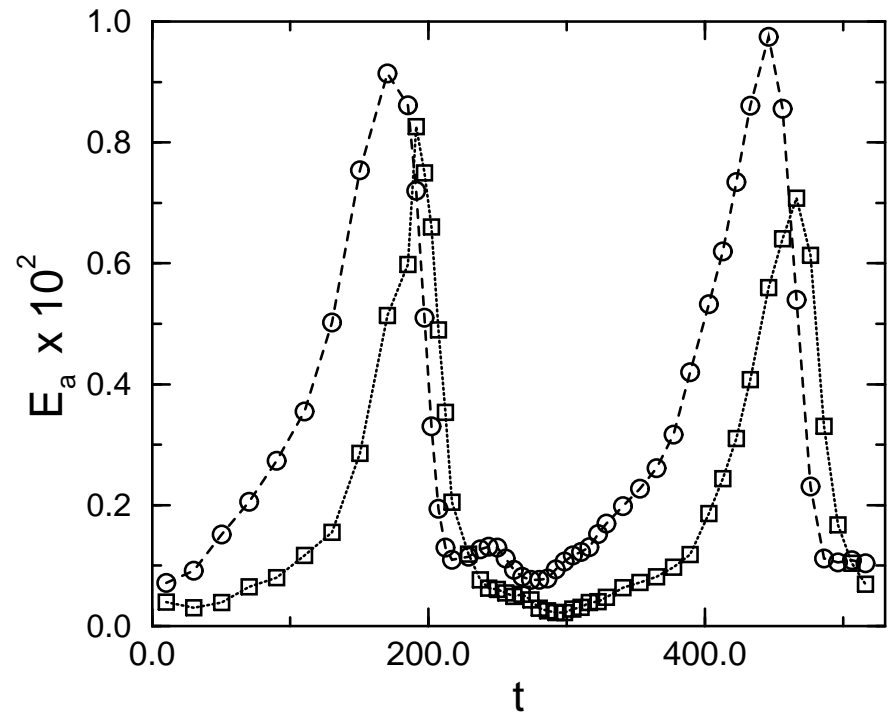

Figure 4.9: Total antysimmetric energy $E_{a}$ (circles) and fraction of $E_{a}$ contained in modes $m^{\prime}>2$ (squares) (these last values have been multiplied by 8 in order to appreciate better the phase shift) as function of time in the final saturated regime. 
reconstruction' process through viscous dissipation of the small scales (this is evident at every minimum in the time evolution of the antisymmetric energy). A cycle of growing instability-saturation and small-scales collapse- large-scales reconstruction is perpetuated and it is fairly consistent with the experimental observations reported in section 4.2.

The numerically calculated growth rate of the instability in its nonlinear evolution is approximately equal to: $\sigma^{(n)} \simeq 0.8 \times 10^{-2}$. This value is about a factor of 2.5 smaller than the initial linear growth rate, showing that the nonlinear phase is governed by other mechanisms than the linear one.

Having in mind geophysical applications, small ellipticities are of interest, therefore one could ask whether these two mechanisms of inertial instability are still effective at geophysically relevant values of the parameters.

Figures 4.2 and 4.3 show actually that several couplings exist even at very small ellipticities to be compared with the Earth's value of the core-ellipticity: $e \simeq 2.45 \times 10^{-3}$.

Since for $e<<1$ resonant couplings involve Poincaré modes which belong to vector spaces $\mathcal{V}_{l^{\prime}}$ with $l^{\prime}>>3$, the viscous damping calculated by (4.21) at Ekman numbers numerically attainable $\left(10^{-4}-5 \times 10^{-5}\right)$ exceeds (or approximately equals) the elliptical or shear growth rates. Note that the two parameters $\varepsilon$ and $\beta$ given by (4.4) decrease with $e$. This turned out to be the major obstacle in detecting numerically an inertial instability at small ellipticity. In spite of that, some critical parameters have been found fixing the Ekman number at the smallest value reachable numerically and looking for a small elliptical distortion of the container at which the elliptical or shear growth rate could exceed the viscous damping.

Choosing:

$$
E=5 \times 10^{-5}, \quad e=0.06, \Omega=-0.14, \alpha=30^{\circ} \text { and } \eta=0.1
$$

the relevant parameters assume the following values:

$$
\nu \simeq s \times 9 \times 10 \times 5 \times 10^{-5} \simeq s \times 4.5 \times 10^{-3}
$$

(for $l^{\prime}=9$ the theory predicts several possibilities for couplings)

$$
\begin{aligned}
& \varepsilon \simeq 3.19 \times 10^{-2} \\
& \beta \simeq 3.13 \times 10^{-2}
\end{aligned}
$$

Since

$$
\sigma_{\varepsilon}^{u p p e r} \simeq 0.6052 \times 3.19 \times 10^{-2} \simeq 1.93 \times 10^{-2}>\nu
$$

an inertial instability could be expected to grow. Incidentally, $\varepsilon \simeq \beta$ which implies an approximately equal probability for the shear and elliptical instability to develop.

Initially, two modes, fulfilling the shear instability resonant conditions, with azimuthal wavenumbers $m^{\prime}=3$ and 4 (with respect to the rotated frame where the $z^{\prime}$-axis coincides with the axis of the fluid) are excited, the symmetric components containing more energy than the antisymmetric ones. Indeed, Poincaré modes (eq.(4.7)) are symmetric or antisymmetric with respect to the reflection through the origin, of a point $(r, \varphi, z)$ relative 


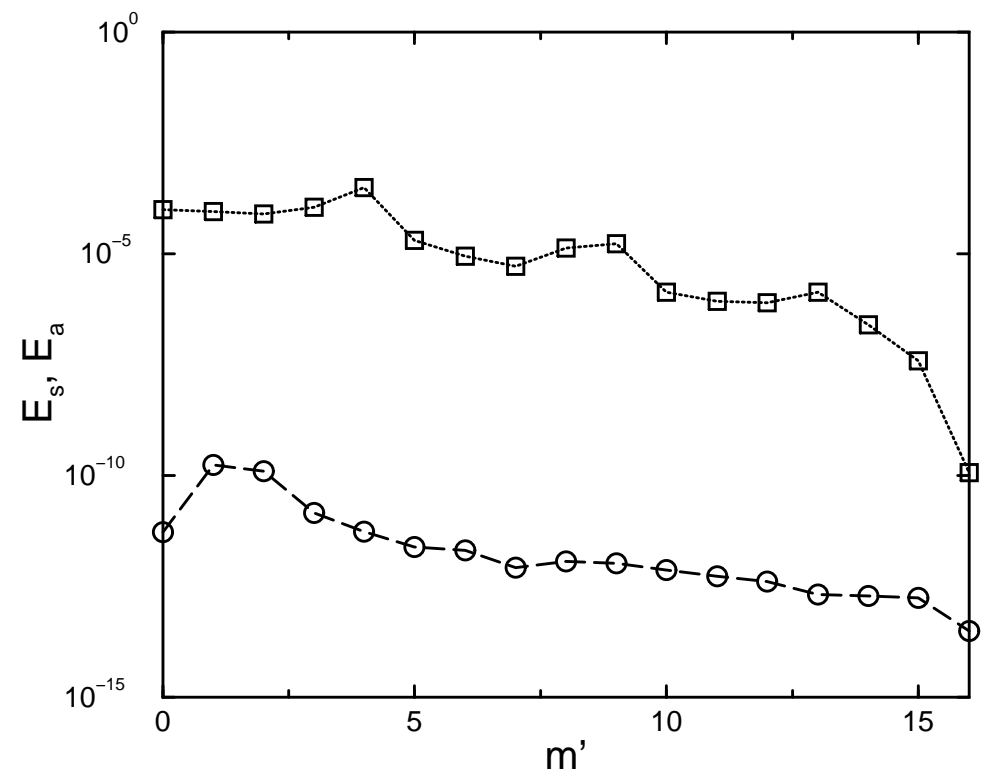

Figure 4.10: Energy contained in the modes with wavenumber $m^{\prime}$ as a function of $m^{\prime}$ for $\eta=0.1, e=0.06, \alpha=30^{\circ}, \Omega=-0.14$ and $E=5 \times 10^{-5}$. Antisymmetric (circles) and symmetric (squares) contributions are shown separately. The antisymmetric spectrum has been multiplied by $10^{5}$.

to a fixed Cartesian coordinate system, depending on whether the modal degree $l^{\prime}$ (introduced in section 4.1) is even or odd, respectively. The linear analytic theory suggests that a coupling between two Poincaré modes with azimuthal wavenumbers 3 and 4 exists at $l^{\prime}=10$. Therefore, the numerical simulations agree well with the theoretical predictions in showing the right parity of the instability. Figure 4.10 displays the $m^{\prime}$-spectrum of the symmetric and antisymmetric energy in the initial linear phase of exponential growth.

In addition to the main instability which resides in the symmetric components, a weaker coupling is also evident between the antisymmetric modes $m^{\prime}=1$ and 2 (which according to the theory should be excited in correspondence of $l^{\prime}=9$ ). The numerically computed linear growth rate is equal to: $\sigma^{(n)} \simeq 0.995 \times 10^{-2}$, that is about a factor of 2 smaller than the linear growth rate calculted in the previous example, consistent with the increased value of the viscous damping.

The cylindrical cuts reported in figure 4.11 confirm the picture of an inertial instability consisting of two sets of rolls developed around the fluid axis.

A constellation of other modes are excited during the subsequent phase: $m^{\prime}=7$ and 8 appear in the symmetric component of the velocity field, while $m^{\prime}=4$ and 5 in the antisymmetric component. At this stage the numerical simulations revealed new possibilities of couplings not predicted by the linear theory. However, in all these cases, 

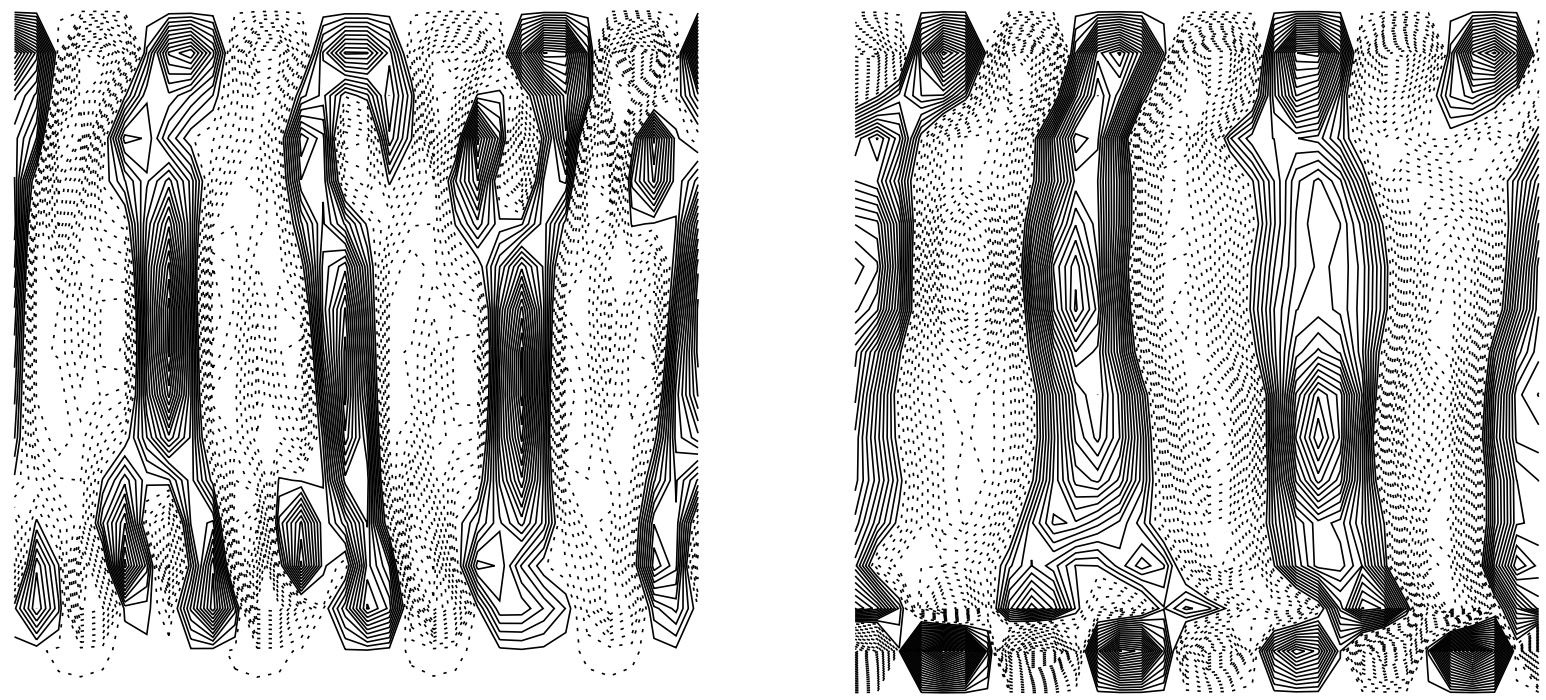

Figure 4.11: $u_{s r}$ (left) and $u_{s z^{\prime}}$ (right) on cylindrical surfaces at distances 0.44 (left) and 0.34 (right) from the $\boldsymbol{\omega}_{F}$-axis for the same parameters as in figure $4.10 . \varphi^{\prime}$ runs from $-\pi$ to $\pi$ in going from left to right and $\vartheta^{\prime}$ runs from -80 to 80 in going from bottom to top.
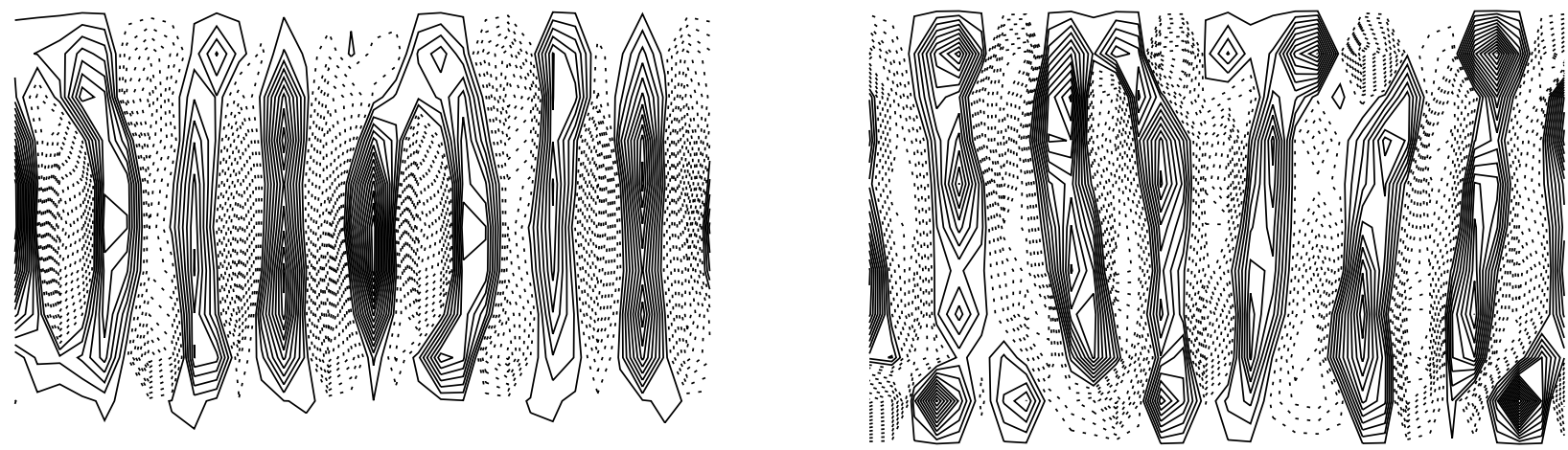

Figure 4.12: $u_{s r}$ (left) and $u_{s z^{\prime}}$ (right) on cylindrical surfaces at distances 0.84 (left) and 0.74 (right) from the $\boldsymbol{\omega}_{F}$-axis for the same parameters as in figure $4.10 . \varphi^{\prime}$ runs from $-\pi$ to $\pi$ in going from left to right and $\vartheta^{\prime}$ runs from -50 to 50 in going from bottom to top. 
two sets of elongated vortices arise. When the instability resides in the antisymmetric components, the rolls which correspond to the odd $m^{\prime}$ are symmetric about the equatorial plane of the primed coordinate system, whereas the columnar vortices associated to the even $m^{\prime}$ are antisymmetric about this plane. The opposite occurs when modes in the symmetric components are excited. Figures $4.12,4.13$ and 4.14 clarify this picture.

During the evolution of this primary instability, a secondary instability has been seen to grow on it, driven by two modes with azimuthal wavenumbers $m^{\prime}=1$ and 3 , fullfilling the elliptical instability resonant conditions.

These two excited inertial waves grow for some time and then dramatically collapse into small-scale disorder.

Previous numerical works performed by Kerswell (1999) and Mason \& Kerswell (1999) in cylindrical geometries have highlighted that a secondary instability can grow on a primary inertial wave instability. A natural candidate for this secondary bifurcation of the whole flow is again a triad resonance in which two infinitesimal inertial waves are coupled by the finite primary wave. At the same time, these authors underlined the ability of geostrophic flows to derive energy through a finite-amplitude inertial wave. The instability with dominant geostrophic component cannot be a triad-type instability since it is easy to prove that a geostrophic flow cannot form a resonant triad with two inertial waves (Greenspan, 1969).

In the following, yet another mechanism for secondary instability is presented: a competitor elliptical instability, whose growth rate was smaller initially, grows to ultimately disrupt the primary excited shearing instability. Figure 4.15 shows the temporal evolution of the antisymmetric energy in the final nonlinear regime. As in the previous example, big-amplitude oscillations appear, even though no more regular in their shape. The growth rate numerically calculated in this nonlinear phase is compatible with the initial linear one: $\sigma^{(n)} \simeq 0.87 \times 10^{-2}$. In figure 4.16 the $m^{\prime}$-spectrum of the antisymmetric energy, calculated at a representative maximum and minimum of the temporal evolution, is presented, whereas figures 4.17 and 4.18 display $u_{a r}$ and $u_{a z^{\prime}}$ in a plane perpendicular to $\boldsymbol{\omega}_{F}$.

From the pictures it is clearly visible that the saturation of the inertial instability causes the whole flow to collapse to small-scale disorder. At every maximum energy is transferred to small scales (breakdown of the flow), which are in turn dissipated by viscosity and a relaminarization process takes place in every minimum. Looking at the spectrum in figure 4.16 (circles), one could suspect that the resolution used $\left(N_{r}=129\right.$, $L=16, M=32$ ) is not adequate due to its flatness. Indeed, this is merely the result of the small-scale excitation which takes place every time the inertial instability saturates. Once the small scales are dissipated by viscosity, the spectrum recovers a decay of several orders of magnitude (see figure 4.16, squares).

In order to simulate the geophysically relevant influence of a solid inner core, an ellipsoidal shell with $\eta=0.35$ has been considered keeping all the other parameters, used in the last example for small ellipticity, fixed. In the linear initial exponential growth phase, the two more strongly excited modes, residing in the symmetric component of the velocity field, have azimuthal wavenumbers $m^{\prime}=3$ and 4 . The $m^{\prime}$-spectrum of the symmetric and 

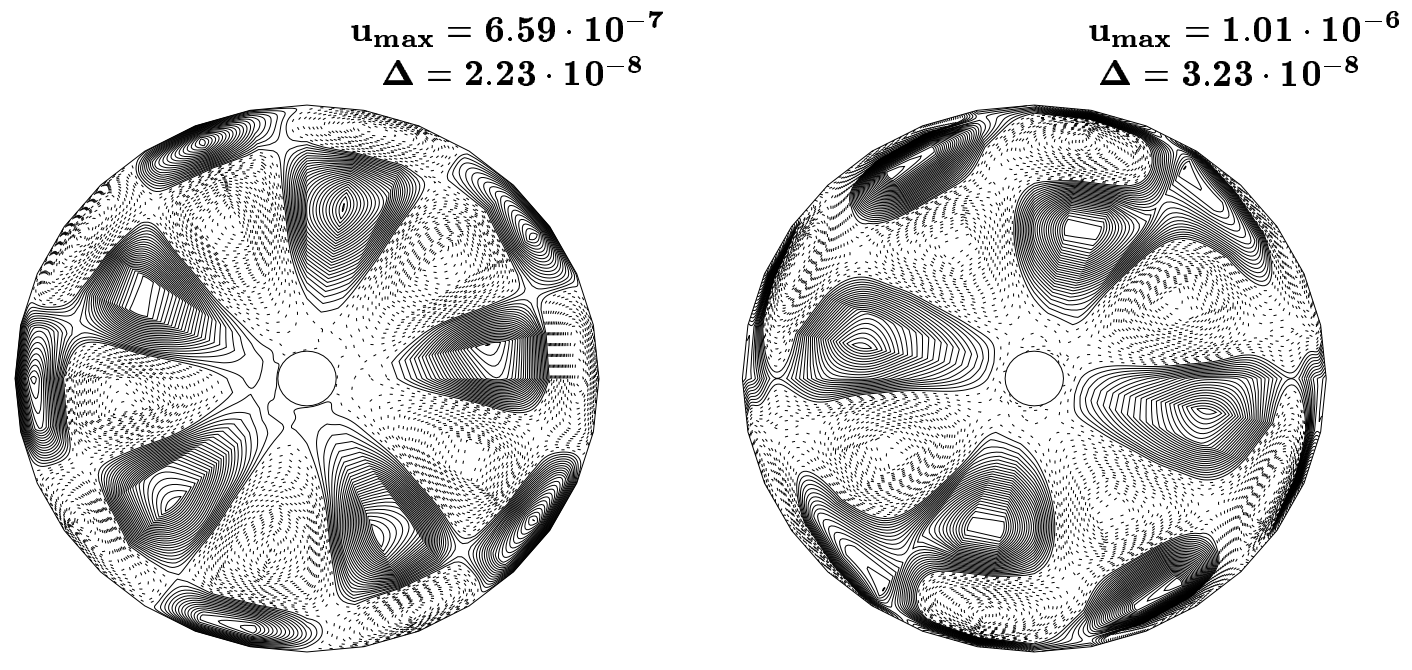

Figure 4.13: $u_{a r}$ (left) and $u_{a z^{\prime}}$ (right) in the plane perpendicular to $\boldsymbol{\omega}_{F}$ for the same parameters as in figure 4.10. Contour level separation $(\Delta)$ and values of the extreme levels $\left(u_{\max }\right)$ are given for both panels.
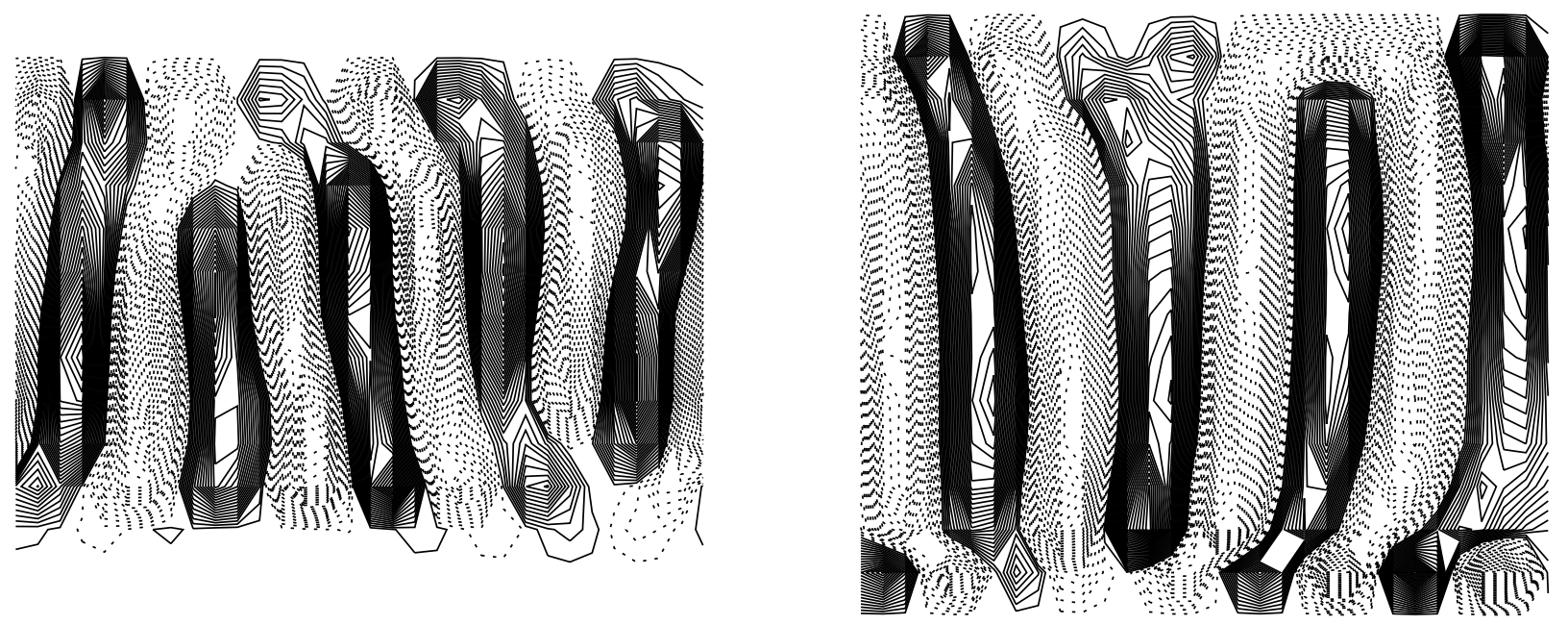

Figure 4.14: $u_{a r}$ (left) and $u_{a z^{\prime}}$ (right) on cylindrical surfaces at distances 0.84 (left) and 0.54 (right) from the $\boldsymbol{\omega}_{F}$-axis for the same parameters as in figure 4.10. $\varphi^{\prime}$ runs from $-\pi$ to $\pi$ in going from left to right and $\vartheta^{\prime}$ runs from -50 to 50 (left) and from -70 to 70 (right) in going from bottom to top. 


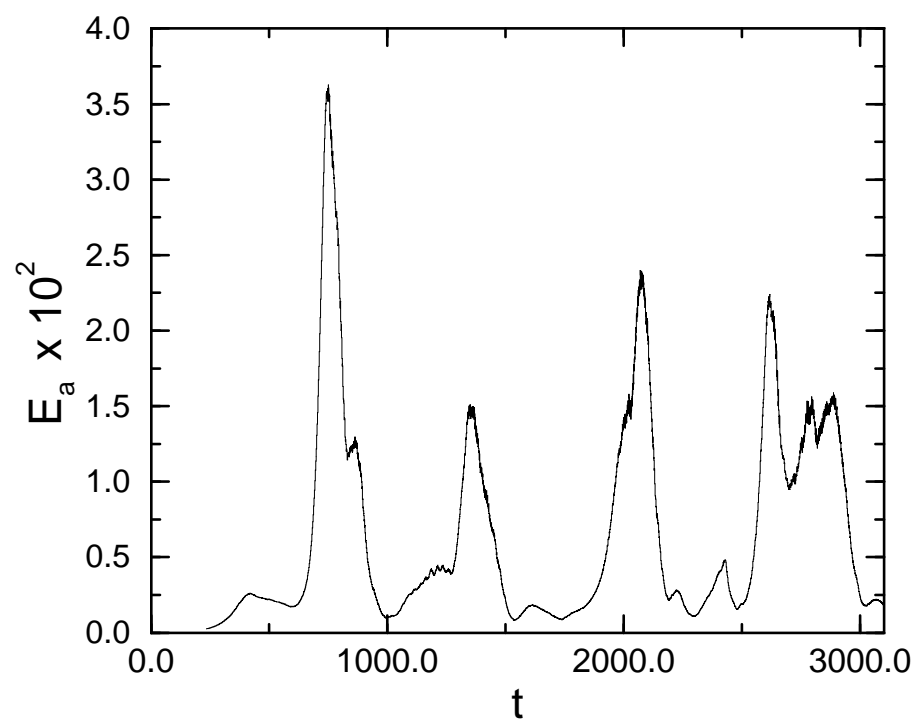

Figure 4.15: Time evolution of the energy $E_{a}$ contained in the velocity components antisymmetric with respect to reflection at the origin for the same parameters as in figure 4.10 .

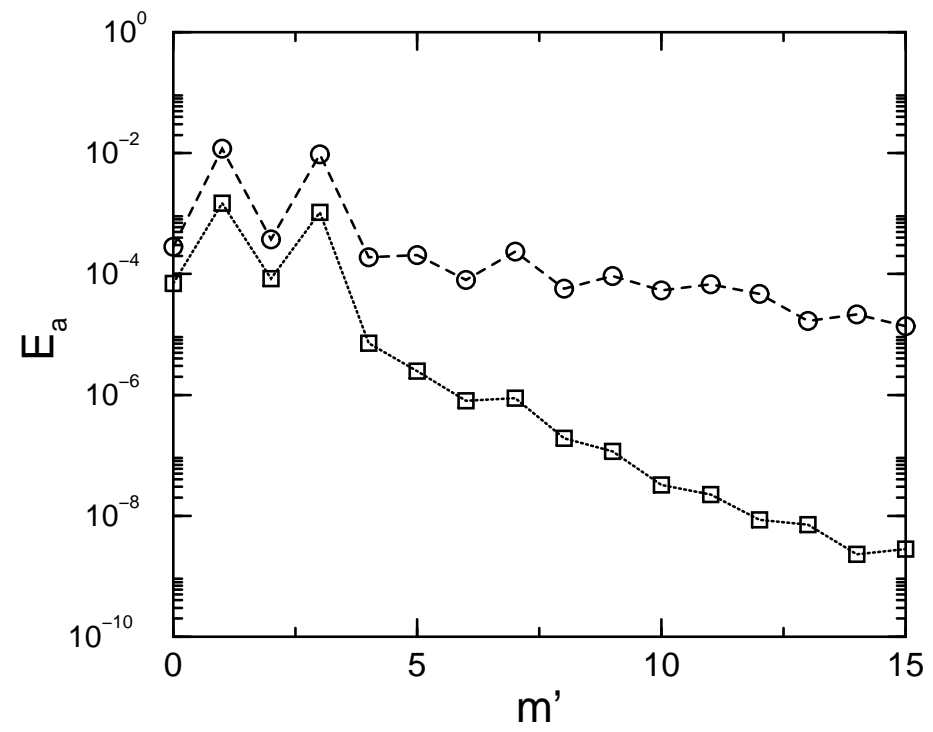

Figure 4.16: Antisymmetric energy contained in the modes with wavenumber $m^{\prime}$ as a function of $m^{\prime}$ for the same parameters of figure 4.10. Circles indicate the spectrum at a maximum, while squares at a minimum in the temporal evolution shown in figure 4.15. 

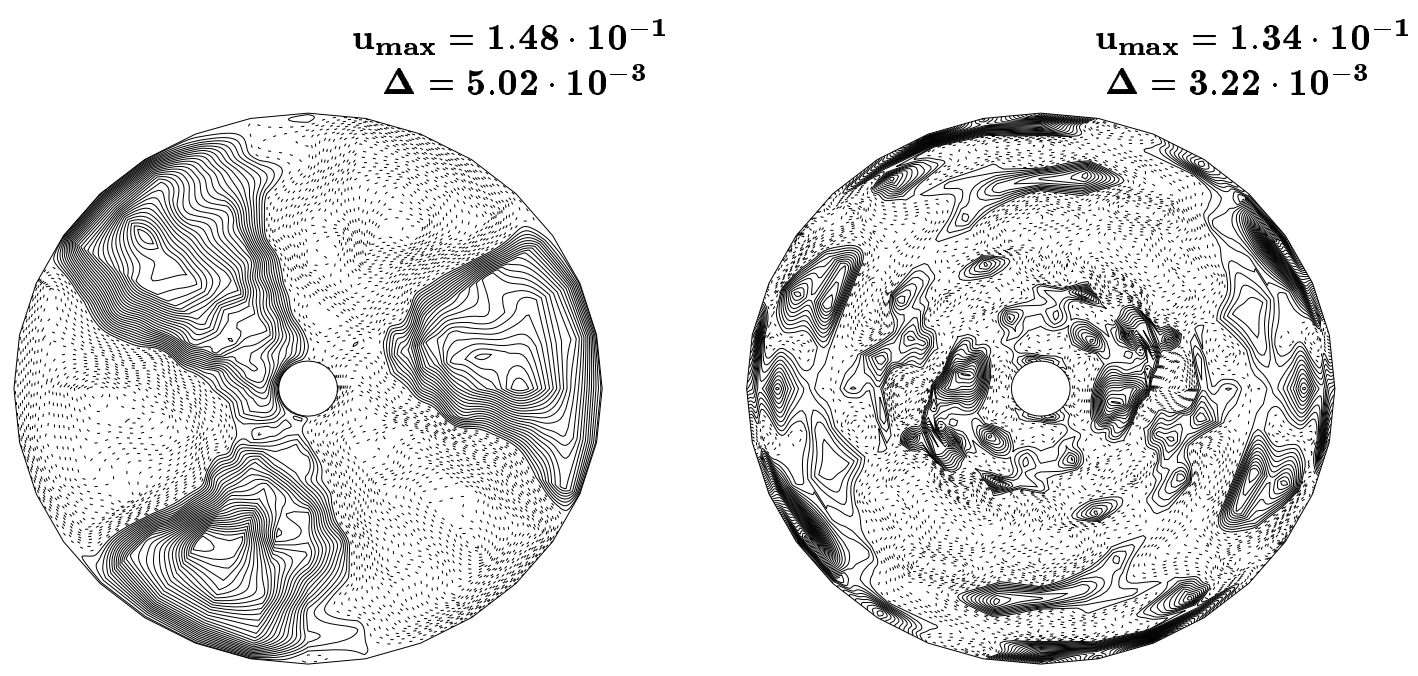

Figure 4.17: $u_{a r}$ (left) and $u_{a z^{\prime}}$ (right) in the plane perpendicular to $\boldsymbol{\omega}_{F}$ for the same parameters as in figure 4.10 , in the final nonlinear phase corresponding to the first maximum appearing in figure 4.15 .
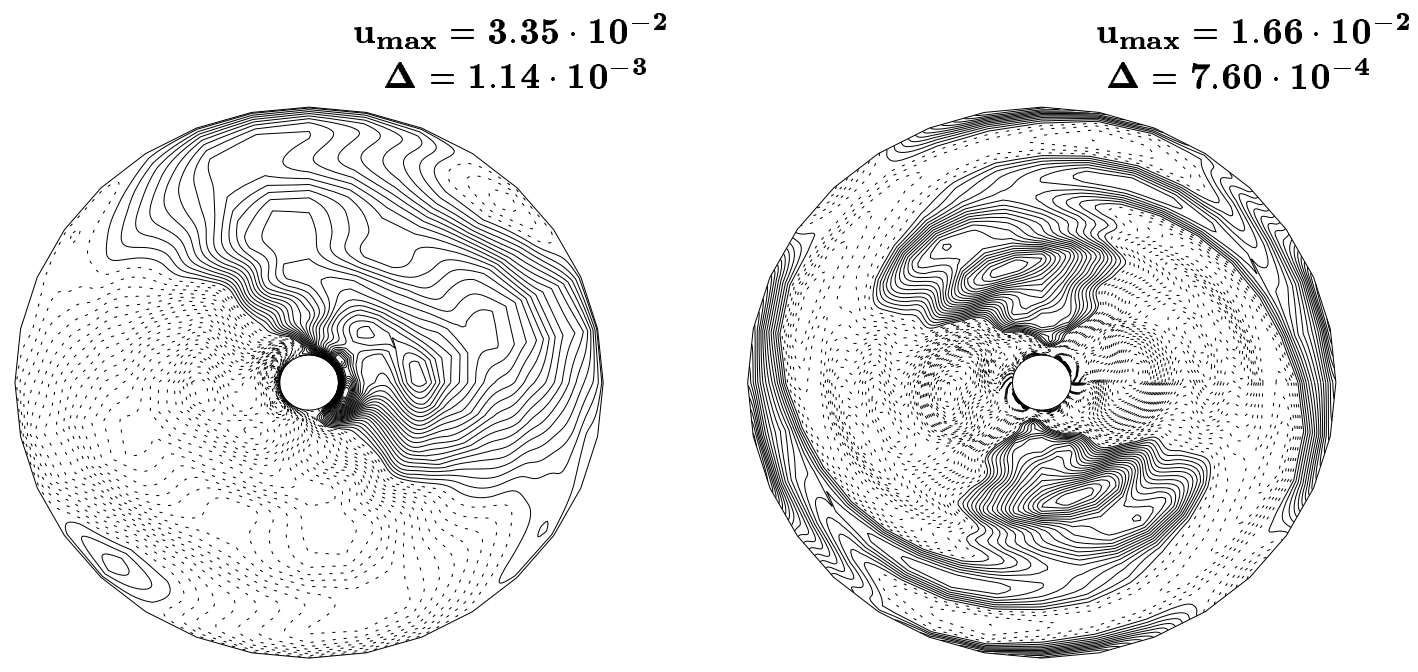

Figure 4.18: $u_{a r}$ (left) and $u_{a z^{\prime}}$ (right) in the plane perpendicular to $\boldsymbol{\omega}_{F}$ for the same parameters as in figure 4.10 , in the final nonlinear phase corresponding to the first minimum appearing in figure 4.15 . 


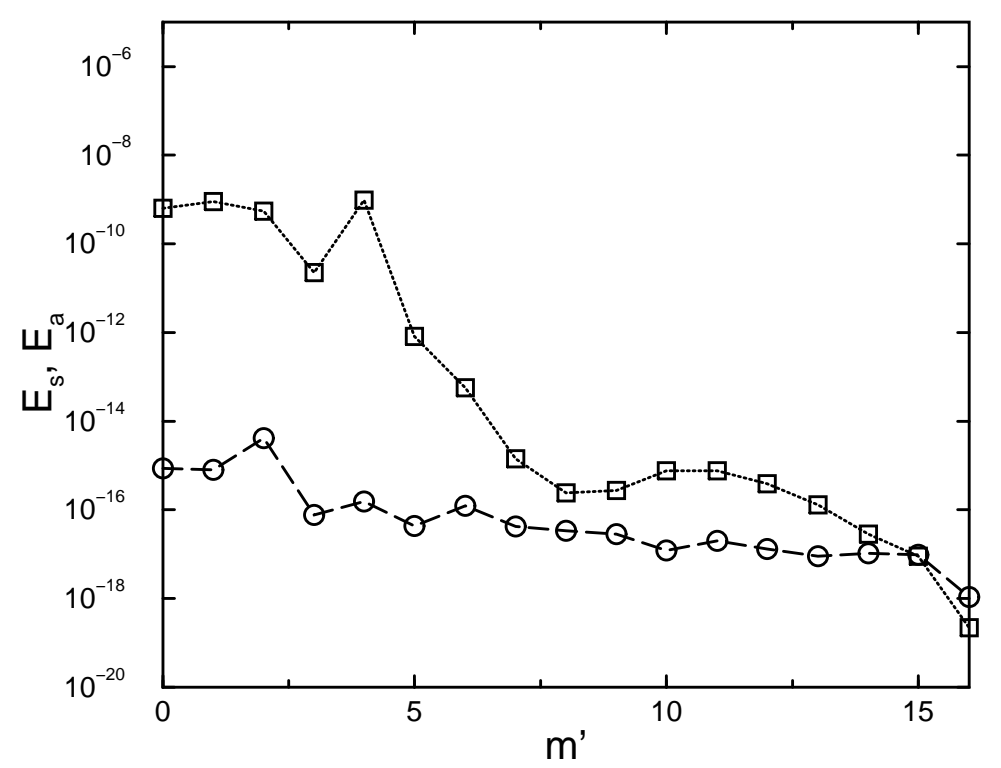

Figure 4.19: Energy contained in the modes with wavenumber $m^{\prime}$ as a function of $m^{\prime}$ for $\eta=0.35, e=0.06, \alpha=30^{\circ}, \Omega=-0.14$ and $E=5 \times 10^{-5}$. Antisymmetric (circles) and symmetric (squares) contributions are shown separately.

antisymmetric energies in this initial linear stage, reported in figure 4.19, resembles very closely the one obtained without inner core (see figure 4.10).

During the subsequent time evolution of the instability, two other modes with azimuthal wavenumbers $m^{\prime}=10$ and 11 are excited, even though the $m^{\prime}$-spectrum of the antisymmetric energy reveals a weaker coupling between $m^{\prime}=4$ and 5 as observed previously without inner core (see figures 4.20 and 4.21).

This can be intuitively explained considering that a shell prevents the development of rolls with small wavenumber which preferentially arise in the internal part of the container close to the axis. The relevant feature is that the instability manifests itself yet in the form of two sets of rolls propagating around the fluid axis, whose linear numerically calculated growth rate is: $\sigma^{(n)} \simeq 1.715 \times 10^{-2}$. This primary instability is again unstable to a secondary elliptical instability driven by the modes $m^{\prime}=1$ and 3 which eventually saturates leading the whole flow to degenerate by the emergence of a turbulent looking pattern. Figure 4.22 reports the three elongated vortices which correspond to the excited $m^{\prime}=3$ mode.

Unlike the other inertial instabilities described so far, no big-amplitude oscillations appear: the saturation state leads to small scale disordered flow which remains unchanged in the subsequent temporal evolution (see figure 4.23).

This behaviour has been recently observed experimentally by C.Eloy, P.Le Gal and S. 


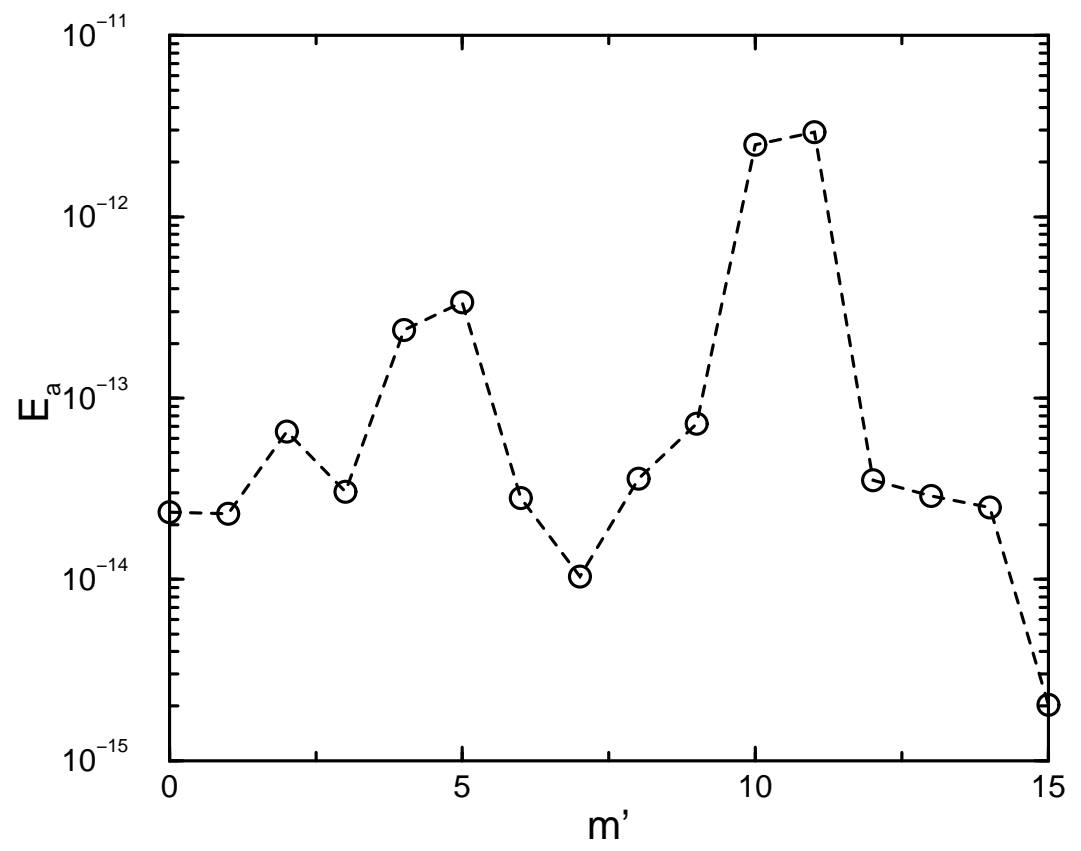

Figure 4.20: Antisymmetric energy contained in the modes with wavenumber $m^{\prime}$ as a function of $m^{\prime}$ for the same parameters as in figure 4.19.
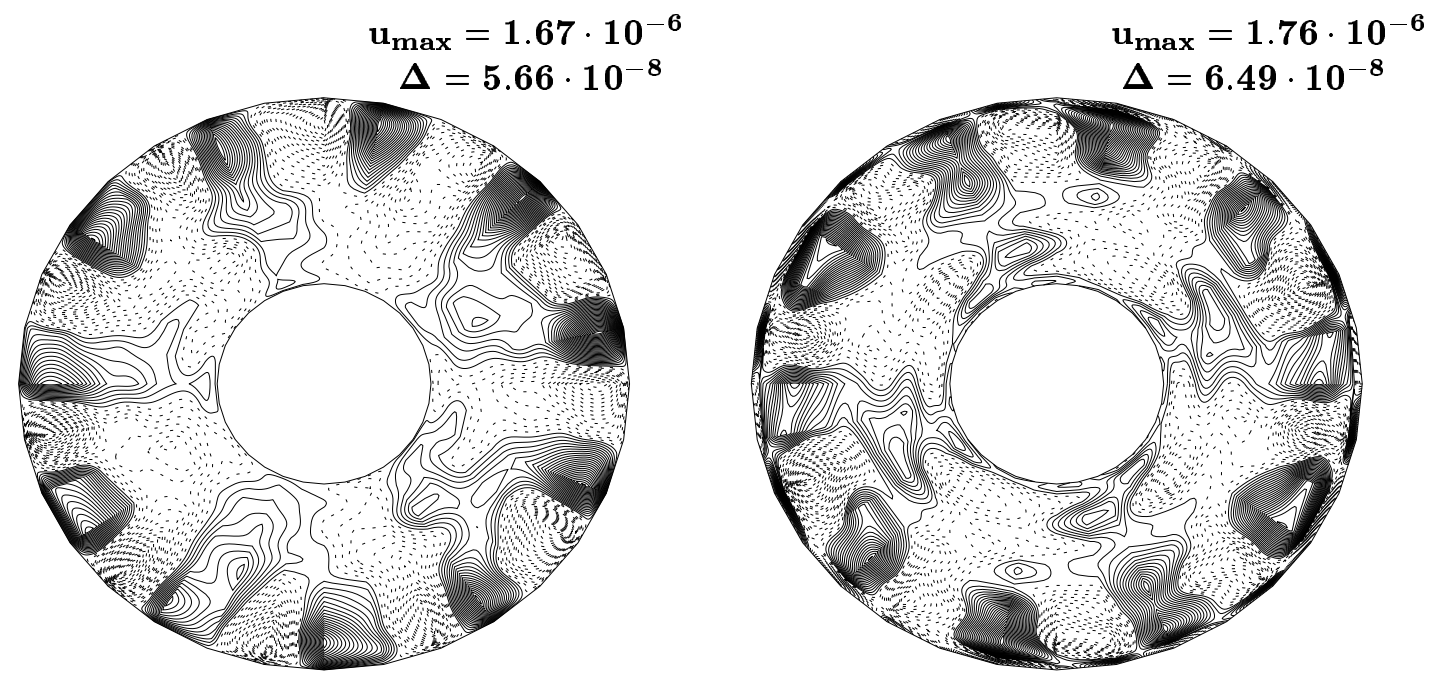

Figure 4.21: $u_{a r}$ (left) and $u_{a z^{\prime}}$ (right) in the plane perpendicular to $\boldsymbol{\omega}_{F}$ for the same parameters as in figure 4.19 . 


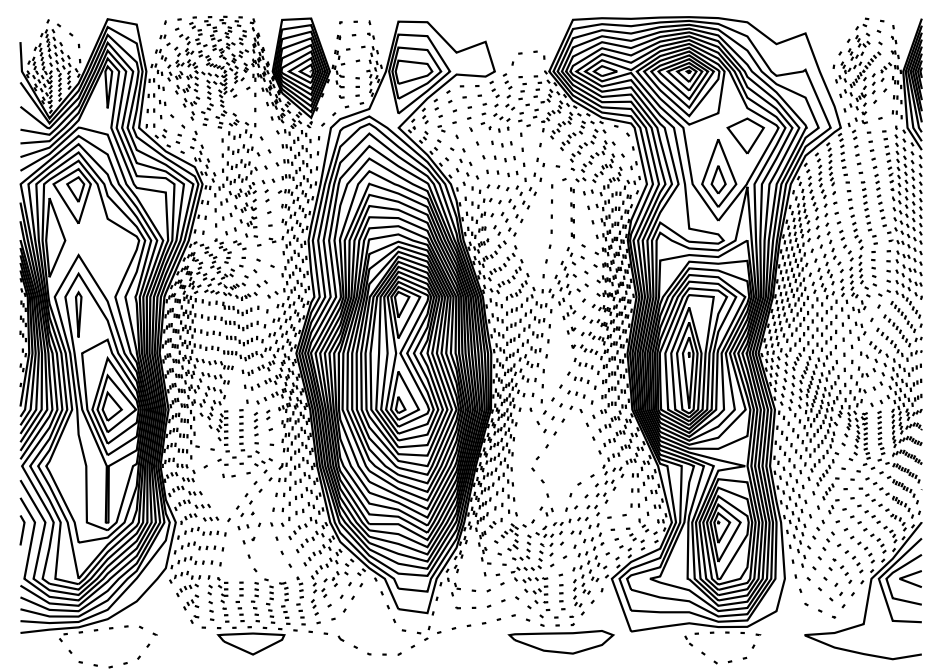

Figure 4.22: $u_{a r}$ on a cylindrical surface at a distance 0.84 from the $\boldsymbol{\omega}_{F}$-axis for the same parameters as in figure 4.19. $\varphi^{\prime}$ runs from $-\pi$ to $\pi$ in going from left to right and $\vartheta^{\prime}$ runs from -50 to 50 in going from bottom to top.
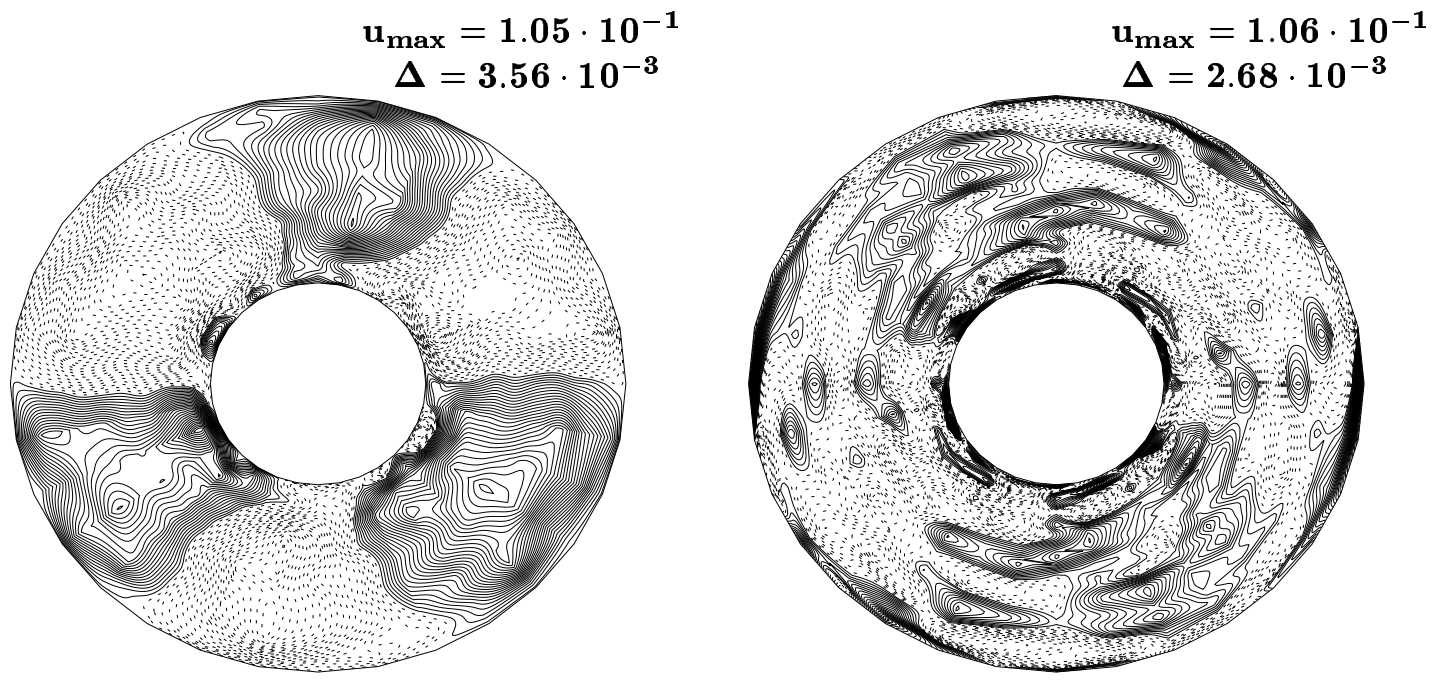

Figure 4.23: $u_{a r}$ (left) and $u_{a z^{\prime}}$ (right) in the plane perpendicular to $\boldsymbol{\omega}_{F}$ for the same parameters as in figure 4.19 in the final nonlinear phase. 
Le Dizés (see section 4.2) as characterizing elliptical instability close to threshold.

\subsection{Hyperviscosity}

So far two different instability mechanisms, leading to bifurcating solutions in precessing ellipsoidal shells, have been detected.

Adopting realistic no-slip boundary conditions, the instability of the internal viscous shear layers emanating from critical latitudes has been studied as well as the instability of the Ekman boundary layers.

In order to capture unstable Poincaré modes responsible for inertial instabilities, one needs to get rid of the unresolvably small scales produced by the boundary layer instability when the Ekman number and consequently the viscosity damping is reduced. This has been done introducing free-slip boundary conditions, which give rise to weak Ekman layers with almost no influence on the dynamics of the interior flow.

Therefore, stress free boundary conditions inhibit the possibility of taking into account fluid flow viscous corrections. On the other hand, no-slip boundary conditions prevent us from reaching small Ekman numbers for a more direct comparison with experiments and with the relevant geophysical context.

Standing the present computer resources with the employed numerical methods, the only available perspective, in order to investigate, if possible, the simultaneous excitations of both the inertial and viscous instabilities, has been traced in using no-slip boundary conditions in conjunction with 'hyperviscosity'. This means that the usual form of the diffusive terms, like viscosity, is replaced by a new form depending on the spherical harmonic degree $l$, in the following way:

$$
\nu(l)=\nu\left[1+\gamma\left(l-l_{o}\right)^{n}\right]
$$

where $l_{o}, \gamma$ and $n$ are fixed parameters. For the present purposes, the following nondimensional expression has been tested as convenient among other possible choices:

$$
E(l)=E\left[1+\frac{0.1}{E}\left(\frac{l}{l_{d}}\right)^{6}\right]
$$

where $l_{d}$ is the highest modal degree considered in the numerical simulations and $E$ the Ekman number. The reason for introducing hyperviscosity is to stabilize numerical schemes: this is done by damping the small scales.

Hyperviscosity is widely used in numerical geodynamo models (for example, Glatzmaier and Roberts, 1995) and in numerical simulations of two-dimensional turbulence and threedimensional turbulence with rotation (Borue and Orszag, 1995).

In order to avoid a too severe influence of hyperviscosity on the dynamics of our system, equation (4.28) has been applied for $l>10$. A direct comparison with runs realized adopting no-slip boundary conditions showed that while hyperviscosity moves only slightly, in the direction of higher precession rates at fixed Ekman numbers, the threshold for the 
onset of the instability, on the other hand it affects in a more substantial way the lengthscales of the unstable modes. This means that in the parametric range surveyed in the present simulations no modes with azimuthal wavenumbers $m^{\prime}>4$ (with respect to the fluid axis) have been excited. On the contrary, table 2.4 in chapter 2 shows that using no-slip boundary conditions the detected instability resides in modes up to $m^{\prime}=7$ and 8. This is due to the fact that hyperviscosity alters the length-scales of the internal shear layers which are responsible for the viscous instability: larger shear structures develop preferentially a wake-like instability with smaller azimuthal wavenumbers which is not strictly localized and can propagate in a broader fraction of the fluid volume (the same behaviour has been observed in section 2.4 increasing the precession rate or the Ekman number).

Having this limitation in mind, figure 4.24 presents two series of simulations in which, at fixed $|\boldsymbol{\Omega}|$, the Ekman number $E$ has been systematically decreased, in ellipsoidal (top panel) and spherical geometry (bottom panel).

From the pictures it is evident that at the smallest Ekman number attainable numerically, $E=2 \times 10^{-5}$, a new effect arises in the ellipsoid which breaks the trend observed at higher $E$ and confirmed in the sphere for all $E$.

Two points, corresponding to the same $E=2 \times 10^{-5}$, are drawn in figure 4.24 (top panel) as representative of the unstable energy. This is better understood looking at figure 4.25 which shows in the ellipsoidal case the shape of the antisymmetric energy for all the chosen Ekman numbers.

At $E=10^{-4}$ regular oscillations appear, as previously noted with no-slip boundaries, close to the onset. Decreasing the Ekman number till $E=4 \times 10^{-5}$, a chaotic time dependence sets in but the disturbances are still characterized by small amplitudes and short periods. At $E=2 \times 10^{-5}$, almost regular big-amplitude and long-period oscillations arise, resembling very closely the shape of the antisymmetric energy observed with freeslip boundary conditions whenever an inertial instability has been detected (see figures 4.8 and 4.15 in section 4.4 ).

Believing not appropriate to describe this temporal evolution of the instability through a simple average, the significantly different values assumed by the energy at a maximum and a minimum have been reported in figure 4.24. In the sphere, at small Ekman numbers, none of these features has been observed. Unfortunately, it was not possible to reach smaller $E$ in order to check if the trend displayed in figure 4.24 is maintained.

As mentioned above, the hyperviscosity changes the length-scales of the unstable motions, therefore it is an useless approach to appeal to the linear analytic theory for a comparison between the coupled wavenumbers excited in the numerical simulations and those predicted analytically for ellipticity $e=0.04$. A further complication, in this case, arises since in section 4.4 has been proven that in the nonlinear growth of an inertial instability modes other than those predicted by the linear theory can be excited. What one could do, instead, is to look at the modes in which the instability resides at $E=4 \times 10^{-5}$. For this Ekman number the observed instability is triggered by the internal viscous shear layers. There is no evidence that in this case an inertial mechanism plays a role, and indeed for these parameters, using free-slip boundaries, no inertial instabilities appeared. 

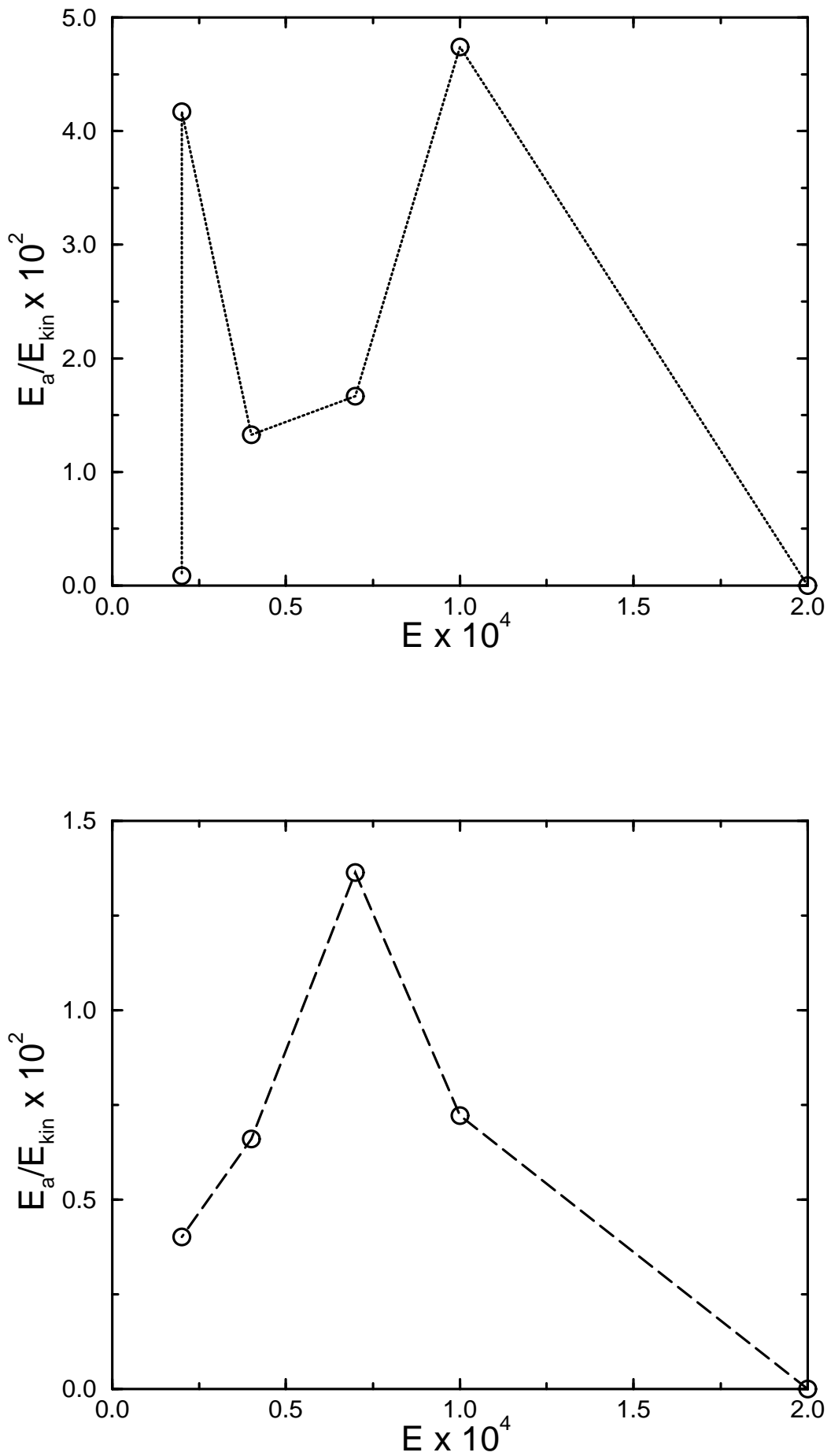

Figure 4.24: The ratio of the antisymmetric energy $E_{a}$ and the total kinetic energy $\tilde{E}_{k i n}$ in components other than $W_{1}^{o}$ and $W_{1}^{1}$ as a function of the Ekman number $E$ for $\Omega=$ $-3.5 \times 10^{-2}, e=0.04, \eta=0.1, \alpha=30^{\circ}$ (top panel) and $\Omega=-0.1, e=0, \eta=0.1$, $\alpha=40^{\circ}$ (bottom panel). 

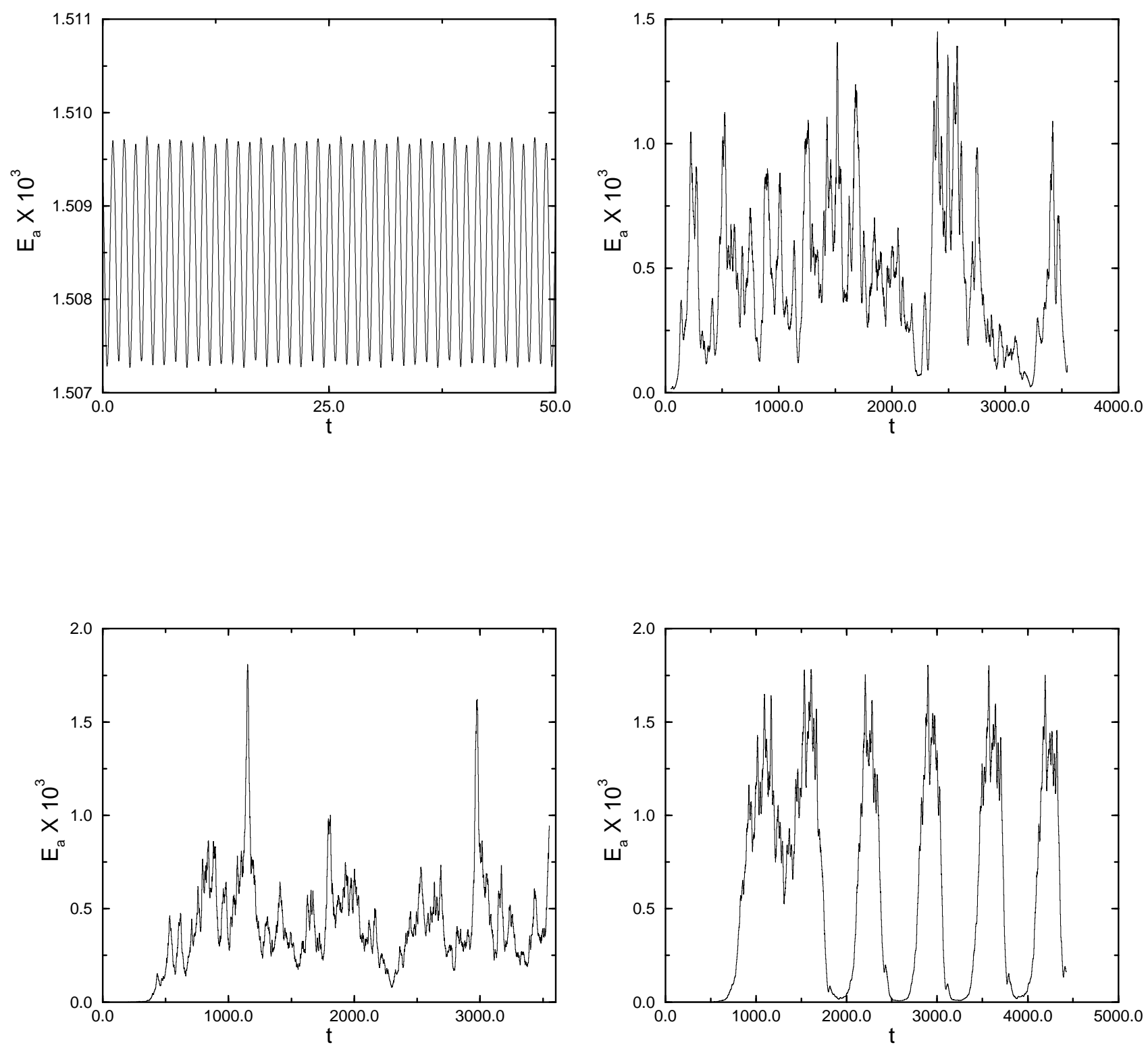

Figure 4.25: Time evolution of the energy $E_{a}$ contained in the velocity components antisymmetric with respect to reflection at the origin for the same parameters as in figure 4.24(top panel) and Ekman numbers: $10^{-4}$ (top-left); $7 \times 10^{-5}$ (top-right); $4 \times 10^{-5}$ (bottom-left); $2 \times 10^{-5}$ (bottom-right). 


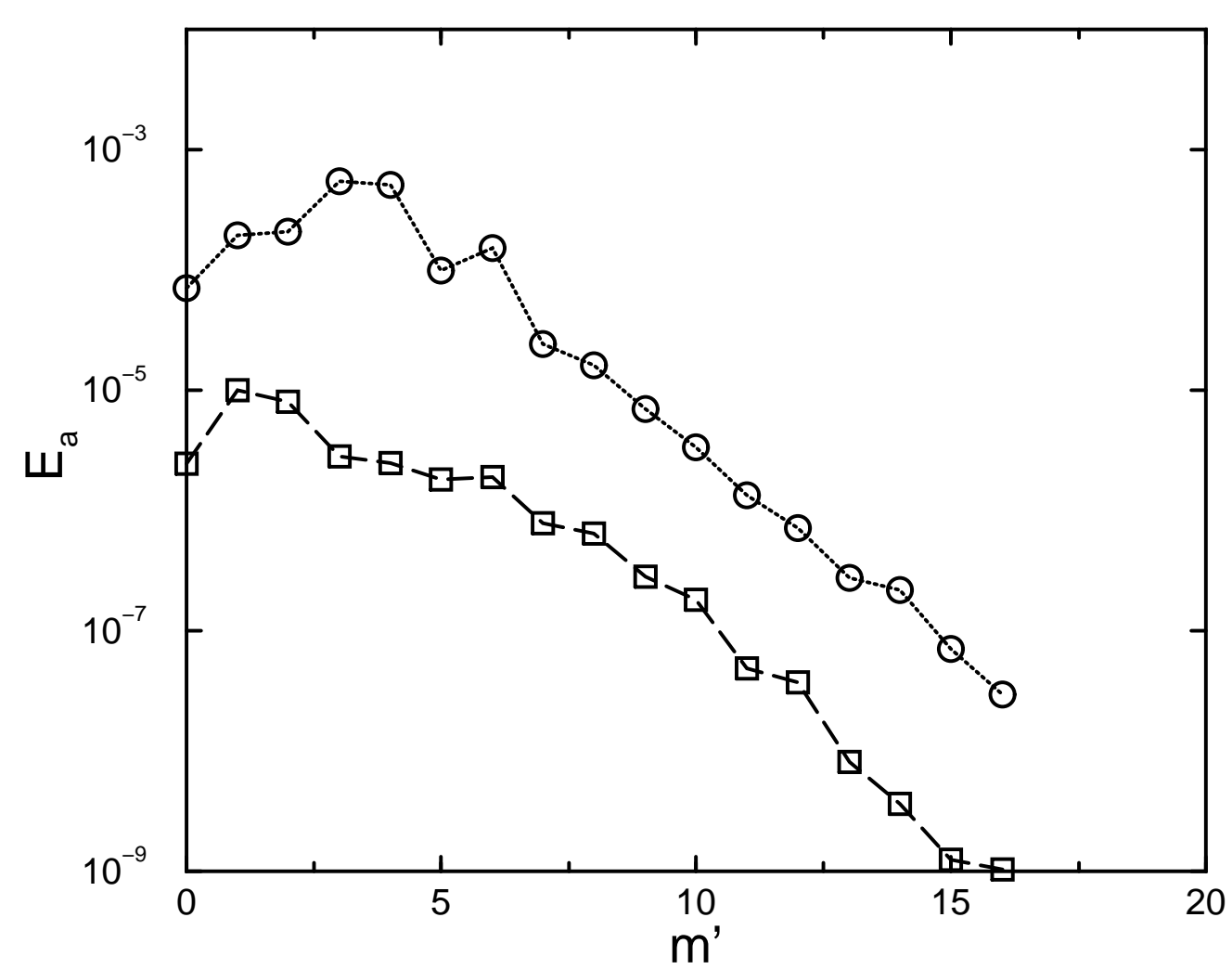

Figure 4.26: Antisymmetric energy contained in the modes with wavenumber $m^{\prime}$ as a function of $m^{\prime}$ for the same parameters of figure 4.24 (top panel) and $E=2 \times 10^{-5}$. Circles indicate the spectrum at a maximum, while squares at a minimum in the temporal evolution shown in figure 4.25 (bottom-right panel).

Going from $E=4 \times 10^{-5}$ to $E=2 \times 10^{-5}$ there is only a factor of 2 which presumably does not affect strongly the intensity of the internal shear layers, therefore one expects that the viscous instability resides in the same wavenumbers.

In figure 4.26 the $m^{\prime}$-spectrum of the antisymmetric energy for $E=2 \times 10^{-5}$, calculated at a representative maximum and minimum of the temporal evolution, is presented.

From the picture it is clearly evident that two different couples of modes are excited at these two points: $m^{\prime}=1$ and 2 appear at every minimum (the same wavenumbers are excited at $E=4 \times 10^{-5}$ ), while $m^{\prime}=3$ and 4 dominate the spectrum at every maximum. A plausible interpretation that one infers from the numerical data is that at small Ekman numbers, in ellipsoidal geometries, both viscous and inertial instabilities can be driven.

The instability of the internal viscous shear layers, consisting of two sets of stable rolls, always accounted for only a small fraction of the total energy. An inertial instability 
can grow on it driving the energy at higher values, which in turn saturates and finally disappears. The cycle perpetuates as time goes on. Although at every maximum the unstable energy is approximately two orders of magnitude bigger than at the corresponding minimum, and the antisymmetric component of the flow is highly disordered, it does not give a significant contribution to the total energy. On the other hand, in the first experiment of Malkus (with $e=0.04$ ), to which the numerical investigations above described are linked, there was no evidence of an abrupt increase of the unstable energy, while the visual observations showed a disorganized flow structure. 


\section{Chapter 5}

\section{Conclusions and perspectives}

The Earth precesses with a period of 25700 years due to the gravity fields of the Moon and the Sun acting on the Earth's equatorial bulge. The influence of the Earth's precessional motion on the dynamics of its liquid outer core has been investigated through direct numerical simulations (DNS). Ellipsoidal cavities with small ellipticities, with and without a sizeable inner core, have been considered in order to reproduce and complement the existing experimental and theoretical findings in the geophysical context.

Previous numerical works have mainly investigated laminar flows in precessing spherical shells and have not been successful beyond linearized equations of motion with ellipsoidal boundaries. The challenge faced in the present research consists in taking into account the effect of nonlinearities which reveals of paramount importance in order to find bifurcating solutions in the transition to hydrodynamic instability.

The solution of the Navier-Stokes equations for inviscid fluids is one of constant vorticity (Poincaré, 1910). Viscosity modifies Poincaré's solution in that thin Ekman boundary layers appear which in turn spawn internal shear layers emanating from critical latitudes (Busse, 1968; Kerswell, 1995; Tilgner, 1999). At low precession rates, when the velocity fields associated with viscous corrections assume small values and the fluid flow is mainly a solid-body rotation around an axis only slightly displaced from the container rotation axis, the flow is laminar. At higher precession rates, the angle between the container and fluid axes increases and the deviations from a solid-body rotation lead to strong non-linear effects.

In this regime, the numerical simulations, presented in chapter 2 , have revealed the instability of the internal viscous shear layers driven by the $m^{\prime}=1$ (with respect to the fluid axis) disturbances of the basic flow. A triad resonance mechanism is responsible for the excitation of this kind of instability which manifests itself in the form of two sets of columnar vortices, with wavenumbers differing by one and nearly equal frequencies, rotating independently of each other and propagating around the fluid axis. The excited motions, which are symmetric with respect to the equatorial plane, remind of Rossby waves. The potential of free shear layers as sources of instability has been pointed out since a long time by Greenspan (1968) and indicated originally by Malkus as the explanation of his experimental results (1968). No qualitative differences have been found in 
spherical shells as concerns the wave-like viscous instability indicating that this mechanism is independent of the shape of the container. Experiments coupled with theory already suggested that the wavy instability exists in the Earth (Malkus, 1971).

Independently of the dynamics of the bulk fluid, the Ekman layers may become unstable. This boundary layer instability accompanied by unresolvably small scales has limited the range of parameters accessible with the employed numerical methods. In the experiments, the boundary layer is very thin and its instability has not been seen. However, such an instability should exist in the Earth.

The data on the orientation of the average vorticity of the flow show that one can apply with confidence the formula of Busse (1968) to the Earth. According to this theory, the angle between the Earth's axis of figure and the rotation axis of the core is $10^{-5}$ which leads to velocities at the core mantle boundary of up to $2.5 \mathrm{~mm} / \mathrm{s}$. Based on a critical Reynolds number for the boundary layer of 100 and using $1.4 E^{1 / 2}$ for the layer thickness, the Earth's Ekman layer must be unstable if the Ekman number of the Earth's core is less than $2 \times 10^{-14}$, which is the case according to current estimates.

Precession-driven flows received over the years a considerable attention in connection with the origin of the Earth's magnetic field. While the kinematic aspects of the Earth's magnetic field generation are now well understood in terms of motions of the conducting fluid in the outer core, the dynamic problem deserves yet a more accurate investigation. Convection is usually regarded as the most likely source of energy for the geodynamo. The precession of the Earth can be seen as an alternative or complementary mechanism. The laminar precession-driven motions are nearly toroidal and consequently not suitable for a dynamo action. Even though more complex turbulent flows could have a better chance in generating a magnetic field, the energy contained in the unstable modes detected numerically at high precession rates has always stayed small compared with the total energy and has not been large enough for dynamo action.

Theory and experiments revealed, however, an energetic inviscid instability mechanism effective in a precessing ellipsoidal cavity due to the elliptical or shearing strains present which elliptically distort the circular streamlines and shear their centres, respectively. These strains can couple two inertial waves through a triad resonance mechanism when the difference in their frequencies coincides with one of the distortion frequencies of the basic flow. In chapter 4 , evidence for the inertial instability has been presented which can be considered a new contribution, beyond the linear analytical theory, in understanding the secondary, nonlinear phases existing after the initial stage of exponential growth. The numerical results show that the form in which an inertial instability manifests itself resembles very closely the one arising in the viscous case: two sets of rolls propagate around the fluid axis. During the evolution of a primary inertial wave instability, a secondary instability can grow on it through a new triad resonance mechanism or as a competitor inertial instability. The unstable coupled inertial modes grow until they saturate and break down: a cascade of energy from large to small scales is therefore realized.

A cycle of growing instability-saturation and small scales collapse-large scale reconstruction is perpetuated and is fairly consistent with the experimental observations when- 
ever inertial waves are driven to finite amplitude. The energy associated with every appearance of an inertial instability has always given a significant contribution to the total energy of the system. While the violent 'resonant collapse' leading to turbulent motion has been widely observed in the experiments, severe difficulties have been encountered in isolating the weakly nonlinear saturated states generated by unstable inertial waves. Therefore, no clear experimental evidence exists concerning the form assumed by a growing inertial disturbance. The numerical simulations presented in section 4.4 , on the contrary, have detected all the phases of evolution of an inertial instability: the initial linear phase well described by the analytical theory, the weakly nonlinear stage in which the two excited modes grow in the form of columnar rolls propagating around the fluid axis, the strongly nonlinear final phase in which a secondary instability possibly grows on the first one leading to a breakdown of the whole flow. During the nonlinear evolution, modes other than those predicted by the linear analytical theory have been excited but in all cases two-dimensional flow structures arise.

The instabilities detected in the present research, the viscous instability (section 2.4) and the inertial one (section 4.4), have always revealed themselves in the form of columnar rolls propagating around the fluid axis. The Proudman-Taylor (PT) theorem (introduced in section 1.1) states that under the assumptions of slow, steady and inviscid motion, the flow of a rotating fluid is two-dimensional. In this context, our simulations can be considered a numerical proof of the validity of the PT theorem under much more general conditions. The inviscid hypothesis is not essential in that viscous corrections drive an instability which generates two-dimensional flow structures. The 'slow motion' requisite can be neglected as well: a growing instability is always the result of nonlinear interactions, which therefore can be taken into account without losing the possibility for a reduction of dimensionality. The steadiness of the flow is again not essential in that an inertial instability generates elongated structures which can be unstable to secondary instabilities and consequently they represent only transient features of the flow motion.

The outcome is that all the three assumptions on which the PT theorem relies can be relaxed obtaining yet the same conclusion. The 'two-dimensionality' reveals to be a much more general property of rotating and precessing flows rather than a possibility subordinated to stringent assumptions.

In spite of the limited computer resources, it was tempting to give an explanation of Malkus' experiments (reported in section 1.4) in terms of the fluid instabilities detected numerically. Indeed, in section 4.5, an investigation of the parametric space very close to Malkus' first experiment, at small Ekman numbers, has been realized using 'hyperviscosity'. The numerical results show that at the smallest Ekman number attainable $\left(2 \times 10^{-5}\right)$, in addition to the viscous instability appearing at higher $E$, a further mechanism sets in, which resembles very closely the inertial one. The antisymmetric component of the flow appears highly disordered, although it does not give a significant contribution to the total energy. On the other hand in the first experiment of Malkus, in which the Ekman number was only one order of magnitude smaller than the numerical one, there was no quantitative evidence of an abrupt increase of the unstable energy, while the visual observations showed a disorganized flow structure. Beyond the turbulent motion of the 
flow, a wave-like instability has been widely observed in Vanyo's experiments (1995) as well as in Malkus' experiments (1968).

An investigation at small Ekman numbers could be very interesting in connection with the dynamics of the Earth's outer core (for the Earth, adopting the current estimate, $E \simeq 10^{-15}$ ). An extrapolation of the numerical results, reported in figure 4.24, for $E \rightarrow 0$ is not straightforward because the trend appearing in the picture is not monotonous.

Unfortunately, standing the present computer resources, it seems not possible to go further in reaching smaller Ekman numbers interesting in the experimental context and for geophysical applications. Future efforts should be focused in this direction and in developing more efficient numerical methods. 


\section{Appendix A}

\section{A practical implementation}

\section{A.1 Spatial discretization}

The expressions $[\hat{\boldsymbol{r}} \cdot \boldsymbol{L}]_{l}^{m}$ and $[\hat{\boldsymbol{r}} \cdot \nabla \times \boldsymbol{L}]_{l}^{m}$, appearing in equations (2.34), (2.35) contain the following terms which have to computed:

$\left[\hat{\boldsymbol{r}} \cdot \nabla \times u_{z} \hat{\boldsymbol{z}}\right]_{l}^{m}, \quad\left[\hat{\boldsymbol{r}} \cdot \nabla \times \nabla \times u_{z} \hat{\boldsymbol{z}}\right]_{l}^{m}, \quad\left[\hat{\boldsymbol{r}} \cdot \partial^{2} / \partial z^{2} \nabla \times \boldsymbol{u}\right]_{l}^{m}, \quad\left[\hat{\boldsymbol{r}} \cdot \partial^{2} / \partial z^{2} \nabla \times \nabla \times \boldsymbol{u}\right]_{l}^{m}$

$\left[\hat{\boldsymbol{r}} \cdot \partial^{2} / \partial z^{2} \nabla \times u_{z} \hat{\boldsymbol{z}}\right]_{l}^{m}, \quad\left[\hat{\boldsymbol{r}} \cdot \partial^{2} / \partial z^{2} \nabla \times \nabla \times u_{z} \hat{\boldsymbol{z}}\right]_{l}^{m}, \quad\left[\hat{\boldsymbol{r}} \cdot \nabla^{2} \nabla \times u_{z} \hat{\boldsymbol{z}}\right]_{l}^{m}, \quad\left[\hat{\boldsymbol{r}} \cdot \nabla^{2} \nabla \times \nabla \times u_{z} \hat{\boldsymbol{z}}\right]_{l}^{m}$

In order to realize that, the following equalities are considered:

$$
\begin{gathered}
{[\hat{\boldsymbol{r}} \cdot \boldsymbol{u}]_{l}^{m}=\frac{l(l+1)}{r} V_{l}^{m}(r)} \\
{[\hat{\boldsymbol{r}} \cdot \nabla \times \boldsymbol{u}]_{l}^{m}=l(l+1) W_{l}^{m}(r)}
\end{gathered}
$$

where $V_{l}^{m}$ and $W_{l}^{m}$ are the poloidal and toroidal scalars of the vector field $\boldsymbol{u}$.

Therefore, a first set of three subroutines computes the following six terms:

$$
\begin{gathered}
P O L_{-} R O T_{-} U \equiv[\operatorname{Pol}(\nabla \times \boldsymbol{u})]_{l}^{m}=\frac{r}{l(l+1)}[\hat{\boldsymbol{r}} \cdot(\nabla \times \boldsymbol{u})]_{l}^{m} \\
T O R_{-} R O T_{-} U \equiv[\operatorname{Tor}(\nabla \times \boldsymbol{u})]_{l}^{m}=\frac{1}{l(l+1)}[\hat{\boldsymbol{r}} \cdot \nabla \times(\nabla \times \boldsymbol{u})]_{l}^{m} \\
P O L_{-} V Z Z \equiv\left[\operatorname{Pol}\left(\nabla \times u_{z} \hat{\boldsymbol{z}}\right)\right]_{l}^{m}=\frac{r}{l(l+1)}\left[\hat{\boldsymbol{r}} \cdot \nabla \times u_{z} \hat{\boldsymbol{z}}\right]_{l}^{m} \\
T O R_{-} V Z Z \equiv\left[\operatorname{Tor}\left(\nabla \times u_{z} \hat{\boldsymbol{z}}\right)\right]_{l}^{m}=\frac{1}{l(l+1)}\left[\hat{\boldsymbol{r}} \cdot \nabla \times \nabla \times u_{z} \hat{\boldsymbol{z}}\right]_{l}^{m} \\
P O L_{-} C O R \equiv[\operatorname{Pol}(\nabla \times(\hat{\boldsymbol{z}} \times \boldsymbol{u}))]_{l}^{m}=\frac{r}{l(l+1)}[\hat{\boldsymbol{r}} \cdot \nabla \times(\hat{\boldsymbol{z}} \times \boldsymbol{u})]_{l}^{m}
\end{gathered}
$$




$$
T O R \_C O R \equiv[\operatorname{Tor}(\nabla \times(\hat{\boldsymbol{z}} \times \boldsymbol{u}))]_{l}^{m}=\frac{1}{l(l+1)}[\hat{\boldsymbol{r}} \cdot \nabla \times \nabla \times(\hat{\boldsymbol{z}} \times \boldsymbol{u})]_{l}^{m}
$$

(where Pol and Tor indicate the poloidal and toroidal component, respectively, of the corresponding terms in the square brackets) taking as input the poloidal and toroidal scalars of a vector field $\boldsymbol{u}$ and giving as output the poloidal and toroidal components of the terms:

$$
(\nabla \times \boldsymbol{u}), \quad\left(\nabla \times u_{z} \hat{\boldsymbol{z}}\right), \quad(\nabla \times(\hat{\boldsymbol{z}} \times \boldsymbol{u}))
$$

through the analytical evaluation of the expressions:

$$
\begin{gathered}
{[\hat{\boldsymbol{r}} \cdot(\nabla \times \boldsymbol{u})]_{l}^{m}, \quad[\hat{\boldsymbol{r}} \cdot \nabla \times(\nabla \times \boldsymbol{u})]_{l}^{m}, \quad\left[\hat{\boldsymbol{r}} \cdot \nabla \times u_{z} \hat{\boldsymbol{z}}\right]_{l}^{m}} \\
{\left[\hat{\boldsymbol{r}} \cdot \nabla \times \nabla \times u_{z} \hat{\boldsymbol{z}}\right]_{l}^{m}, \quad[\hat{\boldsymbol{r}} \cdot \nabla \times(\hat{\boldsymbol{z}} \times \boldsymbol{u})]_{l}^{m}, \quad[\hat{\boldsymbol{r}} \cdot \nabla \times \nabla \times(\hat{\boldsymbol{z}} \times \boldsymbol{u})]_{l}^{m} .}
\end{gathered}
$$

Explicitly, these terms can be written as:

$$
P O L \_R O T_{\_} U=r W_{l}^{m}(r)
$$$$
T O R \_R O T \_U=\frac{l(l+1)}{r^{3}} V_{l}^{m}(r)-\frac{2}{r^{2}} \frac{\partial}{\partial r} V_{l}^{m}(r)-\frac{1}{r} \frac{\partial^{2}}{\partial r^{2}} V_{l}^{m}(r)
$$

$$
\begin{aligned}
P O L_{-} V Z Z= & \frac{r}{l(l+1)}\left[\frac{i m}{r^{2}}\left(\frac{(l+1)(l+2)(l+m+1)}{(2 l+3)}\right) V_{l+1}^{m}(r)+\frac{i m}{r^{2}}\left(\frac{(l+2)(l+m+1)}{(2 l+3)}\right) .\right. \\
& \left(V_{l+1}^{m}(r)+r \frac{\partial}{\partial r} V_{l+1}^{m}(r)\right)+\frac{i m}{r^{2}}\left(\frac{l(l-1)(l-m)}{(2 l-1)}\right) V_{l-1}^{m}(r)- \\
& \left.\frac{i m}{r^{2}}\left(\frac{(l-1)(l-m)}{(2 l-1)}\right) \cdot\left(V_{l-1}^{m}(r)+r \frac{\partial}{\partial r} V_{l-1}^{m}\right)+m^{2} W_{l}^{m}(r)\right]
\end{aligned}
$$

$$
\begin{aligned}
T O R_{-} V Z Z= & \frac{1}{l(l+1)}\left[\frac { 1 } { r ^ { 3 } } \left(\frac{l(l+1)(l-m)(l+m)\left(l^{2}+2 l+1\right)}{(2 l-1)(2 l+1)}+\right.\right. \\
& \left.\frac{l^{3}(l+1)(l-m+1)(l+m+1)}{(2 l+1)(2 l+3)}\right) V_{l}^{m}(r)-\frac{1}{r^{2}}\left(\frac{(l+1)^{2}(l-m)(l+m)}{(2 l-1)(2 l+1)}+\right. \\
& \left.\frac{l^{2}(l-m+1)(l+m+1)}{(2 l+1)(2 l+3)}\right) \cdot\left(2 \frac{\partial}{\partial r} V_{l}^{m}(r)+r \frac{\partial^{2}}{\partial r^{2}} V_{l}^{m}(r)\right)+ \\
& \frac{1}{r^{3}}\left(\frac{l(l+3)(l+m+1)(l+m+2)\left(l^{2}+4 l+3\right)}{(2 l+3)(2 l+5)}\right) V_{l+2}^{m}(r)+ \\
& \frac{1}{r^{2}}\left(\frac{l(l+3)(l+m+2)(l+m+1)}{(2 l+3)}\right) \frac{\partial}{\partial r} V_{l+2}^{m}(r)+
\end{aligned}
$$




$$
\begin{aligned}
& \frac{1}{r}\left(\frac{l(l+3)(l+m+2)(l+m+1)}{(2 l+3)(2 l+5)}\right) \frac{\partial^{2}}{\partial r^{2}} V_{l+2}^{m}(r)+ \\
& \frac{1}{r^{3}}\left(\frac{l(l-2)(l-m)(l-m-1)\left(l^{2}-l-2\right)}{(2 l-3)(2 l-1)}\right) \cdot V_{l-2}^{m}(r)+ \\
& \frac{1}{r^{2}}\left(\frac{(l-2)(l-m)(l-m-1)\left(-2 l^{2}+l+3\right)}{(2 l-3)(2 l-1)}\right) \frac{\partial}{\partial r} V_{l-2}^{m}(r)+ \\
& \frac{1}{r}\left(\frac{(l-2)(l+1)(l-m-1)(l-m)}{(2 l-3)(2 l-1)}\right) \frac{\partial^{2}}{\partial r^{2}} V_{l-2}^{m}(r)- \\
& \frac{i m}{r}\left(\frac{l(l+m+1)(l+3)}{(2 l+3)}\right) W_{l+1}^{m}(r)-i m\left(\frac{l(l+m+1)}{(2 l+3)}\right) \frac{\partial}{\partial r} W_{l+1}^{m}(r)- \\
& \left.\frac{i m}{r}\left(\frac{(l+1)(l-m)(l-2)}{(2 l-1)}\right) W_{l-1}^{m}(r)+i m\left(\frac{(l+1)(l-m)}{(2 l-1)}\right) \frac{\partial}{\partial r} W_{l-1}^{m}(r)\right] \\
& P O L_{-} C O R=-\frac{i m r}{l(l+1)} W_{l}^{m}(r)-\frac{1}{r}\left(\frac{(l+2)(l+m+1)}{(2 l+3)}\right) V_{l+1}^{m}(r)- \\
& \frac{1}{r}\left(\frac{(l+2)(l+m+1)}{(l+1)(2 l+3)}\right) \cdot\left(V_{l+1}^{m}(r)+r \frac{\partial}{\partial r} V_{l+1}^{m}(r)\right)+ \\
& \frac{1}{r}\left(\frac{(l-1)(l-m)}{(2 l-1)}\right) V_{l-1}^{m}(r)-\frac{1}{r}\left(\frac{(l-1)(l-m)}{l(2 l-1)}\right) \text {. } \\
& \left(V_{l-1}^{m}(r)+r \frac{\partial}{\partial r} V_{l-1}^{m}(r)\right) \\
& T O R_{-} C O R=-\frac{1}{r}\left(\frac{(l+2)(l+m+1)}{(2 l+3)}\right) W_{l+1}^{m}(r)-\frac{1}{r^{2}}\left(\frac{(l+2)(l+m+1)}{(l+1)(2 l+3)}\right) . \\
& \left(2 r W_{l+1}^{m}(r)+r^{2} \frac{\partial}{\partial r} W_{l+1}^{m}(r)\right)+\frac{1}{r}\left(\frac{(l-1)(l-m)}{(2 l-1)}\right) W_{l-1}^{m}(r)- \\
& \frac{1}{r^{2}}\left(\frac{(l-1)(l-m)}{l(2 l-1)}\right) \cdot\left(2 r W_{l-1}^{m}(r)+r^{2} \frac{\partial}{\partial r} W_{l-1}^{m}(r)\right)- \\
& \frac{i m}{r^{3}} V_{l}^{m}(r)+\frac{i m}{r^{2} l(l+1)}\left(2 \frac{\partial}{\partial r} V_{l}^{m}(r)+r \frac{\partial^{2}}{\partial r^{2}} V_{l}^{m}(r)\right)
\end{aligned}
$$

A second set of three subroutines can then apply recursively the previous subroutines in order to evaluate the terms entering into the equations $(2.34),(2.35)$ as reported in the following: 


$$
\begin{aligned}
{\left[\hat{\boldsymbol{r}} \cdot \partial^{2} / \partial z^{2} \nabla \times \boldsymbol{u}\right]_{l}^{m}=[\hat{\boldsymbol{r}} \cdot \nabla \times(\hat{\boldsymbol{z}} \times \nabla \times(\hat{\boldsymbol{z}} \times(\nabla \times \boldsymbol{u})))]_{l}^{m}=} & \\
& \frac{l(l+1)}{r} \cdot[\operatorname{Pol}(\nabla \times(\hat{\boldsymbol{z}} \times \nabla \times(\hat{\boldsymbol{z}} \times(\nabla \times \boldsymbol{u}))))]_{l}^{m}= \\
& \frac{l(l+1)}{r} \cdot P O L \_C O R\left[P O L \_ C O R \left(P O L \_R O T \_U,\right.\right. \\
& \left.\left.T O R \_R O T \_U\right), T O R \_C O R\left(P O L \_R O T \_U, T O R \_R O T \_U\right)\right]
\end{aligned}
$$

$$
\begin{aligned}
& {\left[\hat{\boldsymbol{r}} \cdot \partial^{2} / \partial z^{2} \nabla \times \nabla \times \boldsymbol{u}\right]_{l}^{m}=[\hat{\boldsymbol{r}} \cdot \nabla \times \nabla \times(\hat{\boldsymbol{z}} \times \nabla \times(\hat{\boldsymbol{z}} \times(\nabla \times \boldsymbol{u})))]_{l}^{m}=} \\
& l(l+1) \cdot[\operatorname{Tor}(\nabla \times(\hat{\boldsymbol{z}} \times \nabla \times(\hat{\boldsymbol{z}} \times(\nabla \times \boldsymbol{u}))))]_{l}^{m}= \\
& l(l+1) \cdot T O R \_C O R\left[P O L \_ C O R \left(P O L \_R O T \_U\right.\right. \\
&\left.\left.T O R \_R O T \_U\right), T O R \_C O R\left(P O L \_R O T \_U, T O R \_R O T \_U\right)\right]
\end{aligned}
$$

$$
\begin{aligned}
{\left[\hat{\boldsymbol{r}} \cdot \partial^{2} / \partial z^{2} \nabla \times u_{z} \hat{\boldsymbol{z}}\right]_{l}^{m}=\left[\hat{\boldsymbol{r}} \cdot \nabla \times\left(\hat{\boldsymbol{z}} \times \nabla \times\left(\hat{\boldsymbol{z}} \times\left(\nabla \times u_{z} \hat{\boldsymbol{z}}\right)\right)\right)\right]_{l}^{m}=} & \frac{l(l+1)}{r} \cdot\left[\operatorname{Pol}\left(\nabla \times\left(\hat{\boldsymbol{z}} \times \nabla \times\left(\hat{\boldsymbol{z}} \times\left(\nabla \times u_{z} \hat{\boldsymbol{z}}\right)\right)\right)\right)\right]_{l}^{m}= \\
& \frac{l(l+1)}{r} \cdot P O L_{-} C O R\left[P O L _ { - } C O R \left(P O L_{-} V Z Z\right.\right. \\
& \left.\left.T O R_{-} V Z Z\right), T O R_{-} C O R\left(P O L_{-} V Z Z, T O R_{-} V Z Z\right)\right]
\end{aligned}
$$

$$
\begin{aligned}
{\left[\hat{\boldsymbol{r}} \cdot \partial^{2} / \partial z^{2} \nabla \times \nabla \times u_{z} \hat{\boldsymbol{z}}\right]_{l}^{m}=\left[\hat{\boldsymbol{r}} \cdot \nabla \times \nabla \times\left(\hat{\boldsymbol{z}} \times \nabla \times\left(\hat{\boldsymbol{z}} \times\left(\nabla \times u_{z} \hat{\boldsymbol{z}}\right)\right)\right)\right]_{l}^{m}=} \\
l(l+1) \cdot\left[\operatorname{Tor}\left(\nabla \times\left(\hat{\boldsymbol{z}} \times \nabla \times\left(\hat{\boldsymbol{z}} \times\left(\nabla \times u_{z} \hat{\boldsymbol{z}}\right)\right)\right)\right)\right]_{l}^{m}= \\
l(l+1) \cdot T O R_{-} C O R\left[P O L _ { - } C O R \left(P O L_{-} V Z Z\right.\right. \\
\left.\left.T O R_{-} V Z Z\right), T O R_{-} C O R\left(P O L_{-} V Z Z, T O R_{-} V Z Z\right)\right]
\end{aligned}
$$

$$
\begin{gathered}
{\left[\hat{\boldsymbol{r}} \cdot \nabla^{2} \nabla \times u_{z} \hat{\boldsymbol{z}}\right]_{l}^{m}=\frac{l(l+1)}{r^{2}} \cdot\left(2 \frac{\partial}{\partial r}\left[\operatorname{Pol}\left(\nabla \times u_{z} \hat{\boldsymbol{z}}\right)\right]_{l}^{m}+r \frac{\partial^{2}}{\partial r^{2}}\left[\operatorname{Pol}\left(\nabla \times u_{z} \hat{\boldsymbol{z}}\right)\right]_{l}^{m}\right)-} \\
\frac{l^{2}(l+1)^{2}}{r^{3}} \cdot\left[\operatorname{Pol}\left(\nabla \times u_{z} \hat{\boldsymbol{z}}\right)\right]_{l}^{m} \\
{\left[\hat{\boldsymbol{r}} \cdot \nabla^{2}\left(\nabla \times \nabla \times u_{z} \hat{\boldsymbol{z}}\right)\right]_{l}^{m}=\frac{l(l+1)}{r^{2}} \cdot\left(2\left[\operatorname{Tor}\left(\nabla \times u_{z} \hat{\boldsymbol{z}}\right)\right]_{l}^{m}+4 r \frac{\partial}{\partial r}\left[\operatorname{Tor}\left(\nabla \times u_{z} \hat{\boldsymbol{z}}\right)\right]_{l}^{m}+\right.} \\
\left.r^{2} \frac{\partial^{2}}{\partial r^{2}}\left[\operatorname{Tor}\left(\nabla \times u_{z} \hat{\boldsymbol{z}}\right)\right]_{l}^{m}\right)-\frac{l^{2}(l+1)^{2}}{r^{2}} \cdot\left[\operatorname{Tor}\left(\nabla \times u_{z} \hat{\boldsymbol{z}}\right)\right]_{l}^{m}
\end{gathered}
$$


The meaning of this schematic representation can be clarified looking at the computation of the first term as an example.

The poloidal and toroidal scalars of the vector $\boldsymbol{u}$ are given as input to calculate the terms: $P O L \_R O T_{-} U$ and $T O R_{-} R O T_{-} U$.

These last terms are given in succession as input to calculate the terms $P O L_{-} C O R$ and $T O R \_C O R$, which enter finally as input to calculate again the term $P O L \_C O R$.

For the calculation of the non-linear terms in the computational domain, one needs the three components of the vector $\left(\nabla \times u_{z} \hat{\boldsymbol{z}}\right)$ in $(r, \vartheta, \varphi)$-space, which can be expressed analytically as follows:

$$
\begin{aligned}
& \left(\nabla \times u_{z} \hat{\boldsymbol{z}}\right)_{r}=\sum_{m=-\infty}^{\infty} \sum_{l=|m|}^{\infty} m^{2} W_{l}^{m}(r) P_{l}^{m}(\cos \vartheta) e^{i m \varphi}+\frac{i m \cos \vartheta}{r^{2}} l(l+1) \\
& V_{l}^{m}(r) P_{l}^{m}(\cos \vartheta) e^{i m \varphi}-\frac{i m \sin \vartheta}{r^{2}}\left(V_{l}^{m}(r)+r \frac{\partial}{\partial r} V_{l}^{m}(r)\right) . \\
& \frac{d}{d \vartheta} P_{l}^{m}(\cos \vartheta) e^{i m \varphi} \\
& \left(\nabla \times u_{z} \hat{\boldsymbol{z}}\right)_{\vartheta}=\sum_{m=-\infty}^{\infty} \sum_{l=|m|}^{\infty} m^{2} \frac{\cos \vartheta}{\sin \vartheta} \cdot W_{l}^{m}(r) P_{l}^{m}(\cos \vartheta) e^{i m \varphi}+\frac{i m \cos ^{2} \vartheta}{r^{2} \sin \vartheta} \\
& l(l+1) V_{l}^{m}(r) P_{l}^{m}(\cos \vartheta) e^{i m \varphi}-\frac{i m \cos \vartheta}{r^{2}}\left(V_{l}^{m}(r)+\right. \\
& \left.r \frac{\partial}{\partial r} V_{l}^{m}(r)\right) \cdot \frac{d}{d \vartheta} P_{l}^{m}(\cos \vartheta) e^{i m \varphi} \\
& \left(\nabla \times u_{z} \hat{\boldsymbol{z}}\right)_{\varphi}=\sum_{m=-\infty}^{\infty} \sum_{l=|m|}^{\infty} \frac{m^{2} \cos \vartheta}{r \sin \vartheta}\left(\frac{1}{r} V_{l}^{m}(r)+\frac{\partial}{\partial r} V_{l}^{m}(r)\right) P_{l}^{m}(\cos \vartheta) . \\
& e^{i m \varphi}+i m \sin \vartheta\left(W_{l}^{m}(r)+r \frac{\partial}{\partial r} W_{l}^{m}(r)\right) P_{l}^{m}(\cos \vartheta) e^{i m \varphi}+ \\
& i m \cos \vartheta W_{l}^{m}(r) \frac{d}{d \vartheta} P_{l}^{m}(\cos \vartheta) e^{i m \varphi}+\frac{\sin \vartheta \cos \vartheta}{r} l(l+1) \cdot \\
& \left(\frac{1}{r} V_{l}^{m}(r)-2 \frac{\partial}{\partial r} V_{l}^{m}(r)\right) P_{l}^{m}(\cos \vartheta) e^{i m \varphi}+\frac{\sin ^{2} \vartheta}{r^{2}} . \\
& \left(r^{2} \frac{\partial^{2}}{\partial r^{2}} V_{l}^{m}(r)+r \frac{\partial}{\partial r} V_{l}^{m}(r)-V_{l}^{m}(r)\right) \frac{d}{d \vartheta} P_{l}^{m}(\cos \vartheta) e^{i m \varphi}- \\
& \frac{\cos ^{2} \vartheta}{r^{2}} l(l+1) V_{l}^{m}(r) \frac{d}{d \vartheta} P_{l}^{m}(\cos \vartheta) e^{i m \varphi}
\end{aligned}
$$

\section{A.2 Temporal discretization}

The criterion used to choose the number of iterations in Jacobi's method will be briefly illustrated in the following. 
Only the diffusive part of the general equation of motion (2.28) will be considered here (which has been implicitly discretized in the full system):

$$
\begin{array}{r}
\frac{\partial}{\partial t} \nabla \times \boldsymbol{u}-E \nabla^{2} \nabla \times \boldsymbol{u}+e(e-2) \frac{\partial}{\partial t}\left(\nabla \times u_{z} \hat{\boldsymbol{z}}\right)-E(e(e-2)) \nabla^{2} \nabla \times u_{z} \hat{\boldsymbol{z}}+ \\
E \frac{e(e-2)}{(1-e)^{2}} \frac{\partial^{2}}{\partial z^{2}}(\nabla \times \boldsymbol{u})+E\left(\frac{e(e-2)}{(1-e)}\right)^{2} \frac{\partial^{2}}{\partial z^{2}} \nabla \times u_{z} \hat{\boldsymbol{z}}=0
\end{array}
$$

This equation can be schematically written as follows:

$$
\boldsymbol{A} \cdot \boldsymbol{x}=\boldsymbol{b}
$$

Splitting $\boldsymbol{A}$ as: $\boldsymbol{A}=\boldsymbol{L}+\boldsymbol{D}+\boldsymbol{U}$, where $\boldsymbol{D}$ is the diagonal part of $\boldsymbol{A}, \boldsymbol{L}$ is the lower triangle of $\boldsymbol{A}$ with zeros on the diagonal, and $\boldsymbol{U}$ is the upper triangle of $\boldsymbol{A}$ with zeros on the diagonal, the $(n+1)$-th step of iteration for Jacobi's method has the form:

$$
\boldsymbol{D} \cdot \boldsymbol{x}^{(n+1)}=-(\boldsymbol{L}+\boldsymbol{U}) \cdot \boldsymbol{x}^{(n)}+\boldsymbol{b}
$$

Since the first two terms of (A.1) decouple in $l$ and $m$, the relative matrix is block diagonal and it can be easily inverted.

Operating with $\hat{\boldsymbol{r}}$. and $\hat{\boldsymbol{r}} \cdot \nabla \times$ on (A.1) and considering an implicit discretization for the second and fourth terms, the two equations for the toroidal and poloidal components, to be treated with Jacobi's method, read respectively:

$$
\begin{aligned}
& W_{l}^{m(n+1)}(t+h)-h E\left(\frac{\partial^{2}}{\partial r^{2}}+\frac{4}{r} \frac{\partial}{\partial r}+\frac{2-l(l+1)}{r^{2}}\right) W_{l}^{\left.m^{(} n+1\right)}(t+h)= \\
& -\frac{1}{l(l+1)}\left(e(e-2)\left[\hat{\boldsymbol{r}} \cdot\left(\nabla \times u_{z} \hat{\boldsymbol{z}}\right)\right]_{l}^{m(n)}(t+h)-\right. \\
& \left.h E(e(e-2))\left[\hat{\boldsymbol{r}} \cdot \nabla^{2} \nabla \times u_{z} \hat{\boldsymbol{z}}\right]_{l}^{m^{(}(n)}(t+h)\right)+ \\
& \quad \frac{1}{l(l+1)} \boldsymbol{b}_{\text {tor }} \\
& \left(\frac{\partial^{2}}{\partial r^{2}}+\frac{2}{r} \frac{\partial}{\partial r}-\frac{l(l+1)}{r^{2}}\right) V_{l}^{\left.m^{(} n+1\right)}(t+h)- \\
& h E\left(\frac{\partial^{2}}{\partial r^{2}}+\frac{2}{r} \frac{\partial}{\partial r}-\frac{l(l+1)}{r^{2}}\right)^{2} V_{l}^{\left.m^{(} n+1\right)}(t+h)= \\
& \frac{r}{l(l+1)}\left(e(e-2)\left[\hat{\boldsymbol{r}} \cdot \nabla \times\left(\nabla \times u_{z} \hat{\boldsymbol{z}}\right)\right]_{l}^{m^{(}(n)}(t+h)-\right. \\
& \left.h E(e(e-2))\left[\hat{\boldsymbol{r}} \cdot \nabla^{2} \nabla \times\left(\nabla \times u_{z} \hat{\boldsymbol{z}}\right)\right]_{l}^{m^{(n)}}(t+h)\right)- \\
& \frac{r}{l(l+1)} \boldsymbol{b}_{p o l}
\end{aligned}
$$


Choosing, as input, time independent poloidal and toroidal scalars which satisfy the no-slip boundary conditions, the terms $\boldsymbol{b}_{t o r}$ and $\boldsymbol{b}_{p o l}$ can be exactly computed through (A.2) and (A.3).

With this assigned $\boldsymbol{b}$ vector, the iterated solution of equations (A.2) and (A.3) can be evaluated, using an initial seed as starting point for the iterations.

The relative error between the iterated solutions, $W_{l}^{m(i t e r)}$ and $V_{l}^{m(i t e r)}$, and the input scalars, $W_{l}^{m}$ and $V_{l}^{m}$, has been calculated as:

$$
\left(\sum_{r, l, m} \frac{\left(W_{l}^{m(i t e r)}(r)-W_{l}^{m}(r)\right)^{2}}{\left(W_{l}^{m}(r)\right)^{2}}\right)^{1 / 2}
$$

(which is the usual Frobenius norm for matrices) and an analogous expression is valid for the poloidal component.

The number of iterations necessary to reduce this relative error to $10^{-7}-10^{-8}$ (a better agreement is not useful in that also the numerical computation of the terms in (A.1) can not reach a major accuracy) has been used in the implementation of Jacobi's method described in chapter 2 to solve the full system of equations.

For small ellipticities of geophysical interest, the method converges enough rapidly (for the typical ellipticities considered, $e=0.04$ and $0.1,5$ and 8 iterations respectively have been used) while the Jacobi iteration diverges for $e>0.2$.

\section{A.3 Parallel implementation}

The numerical code has been parallelized using MPI (Message Passing Programming). The main point of the message-passing paradigm is that the processes communicate by sending each other messages.

The interprocessor communication consumes an acceptable amount of time when the CRAY shmem-routines are used.

The speed-up factor is 95 for 129 processors.

Most of the time, the data are stored such that grid points located at equal radius are stored on identical nodes.

For the computation of the radial derivatives and the implicit time step, however, gridpoints corresponding to the same $m$ need to be stored at the same processor, therefore two subroutines have been implemented in order to switch the direction of parallelization from radial to angular and vice versa.

Since the cosine transform used to switch between real space and Chebychev coefficients performs best if the number of radial grid points is a power of two plus one, the present numerical code runs fastest on a number of processors which is also a power of two plus one.

Time integrations are typically chopped into jobs executing 5000 time steps which consume 3.22 hours on 129 nodes with the following resolution: $N_{r}=129, L=128, M=$ 64. 


\section{Appendix B}

\section{Energies in ellipsoidal cavities}

The total kinetic energy in non-dimensional form, computed directly in physical space, is given by:

$$
\begin{aligned}
& E_{k i n}=\frac{1}{2} \int \tilde{\boldsymbol{u}}^{2} d \tilde{V}=\frac{1}{2} \int\left[\left(u_{r} \sin \vartheta+u_{\vartheta} \cos \vartheta\right)^{2}+(1-e)^{2}\left(u_{r} \cos \vartheta-u_{\vartheta} \sin \vartheta\right)^{2}+u_{\varphi}^{2}\right] \\
& r^{2} \sin \vartheta d r d \vartheta d \varphi
\end{aligned}
$$

The different contributions to the total energy given by the toroidal and poloidal scalars and by the axisymmetric and non-axisymmetric components are conveniently expressed in $r, l, m$-space as follows:

$$
\begin{aligned}
& E_{\text {pol }}^{\text {non-axisym }}=4 \pi \int d r \sum_{l} \sum_{m=1}^{l} \frac{l(l+1)}{(2 l+1)} \frac{(l+m) !}{(l-m) !}\left[l(l+1)\left|V_{l}^{m}\right|^{2}+\left|V_{l}^{m}+r \frac{\partial V_{l}^{m}}{\partial r}\right|^{2}\right]+ \\
& {\left[(1-e)^{2}-1\right] \cdot 4 \pi \int d r \sum_{l} \sum_{m=1}^{l} \frac{1}{(2 l+1)} \frac{(l+m) !}{(l-m) !} \mid \frac{l(l-1)(l-m)}{(2 l-1)} V_{l-1}^{m}+} \\
&\left.\frac{(l+1)(l+2)(l+m+1)}{(2 l+3)} V_{l+1}^{m}\right|^{2}+\left[(1-e)^{2}-1\right] \cdot 4 \pi \int d r \sum_{l} \sum_{m=1}^{l} \frac{1}{(2 l+1)} . \\
& \frac{(l+m) !}{(l-m) !} \mid \frac{(l-1)(l-m)}{(2 l-1)}\left(V_{l-1}^{m}+r \frac{\partial V_{l-1}^{m}}{\partial r}\right)-\frac{(l+m+1)(l+2)}{(2 l+3)} . \\
&\left.\left(V_{l+1}^{m}+r \frac{\partial V_{l+1}^{m}}{\partial r}\right)\right|^{2}+\left[2-2(1-e)^{2}\right] \cdot 4 \pi \int d r \sum_{l} \sum_{m=1}^{l} \frac{1}{(2 l+1)} \frac{(l+m) !}{(l-m) !} . \\
&\left|\frac{l(l-1)(l-m)}{(2 l-1)} V_{l-1}^{m}+\frac{(l+1)(l+2)(l+m+1)}{(2 l+3)} V_{l+1}^{m}\right| \cdot \mid \frac{(l-1)(l-m)}{(2 l-1)} . \\
&\left(V_{l-1}^{m}+r \frac{\partial V_{l-1}^{m}}{\partial r}\right)-\frac{(l+2)(l+m+1)}{(2 l+3)}\left(V_{l+1}^{m}+r \frac{\partial V_{l+1}^{m}}{\partial r}\right) \mid
\end{aligned}
$$




$$
\begin{aligned}
& E_{\text {tor }}^{\text {non-axisym }}=4 \pi \int d r \sum_{l} \sum_{m=1}^{l} \frac{l(l+1)}{(2 l+1)} \frac{(l+m) !}{(l-m) !} r^{4}\left|W_{l}^{m}\right|^{2}+ \\
& {\left[(1-e)^{2}-1\right] \cdot 4 \pi \int d r \sum_{l} \sum_{m=1}^{l} \frac{m^{2}}{(2 l+1)} \frac{(l+m) !}{(l-m) !} r^{4}\left|W_{l}^{m}\right|^{2}} \\
& E_{\text {pol }}^{a x i s y m}=2 \pi \int d r \sum_{l} \frac{l(l+1)}{(2 l+1)}\left[l(l+1)\left|V_{l}^{0}\right|^{2}+\left|V_{l}^{0}+r \frac{\partial V_{l}^{0}}{\partial r}\right|^{2}\right]+ \\
& {\left[(1-e)^{2}-1\right] \cdot 2 \pi \int d r \sum_{l} \frac{1}{(2 l+1)} \mid \frac{l^{2}(l-1)}{(2 l-1)} V_{l-1}^{0}+} \\
& \left.\frac{(l+1)^{2}(l+2)}{(2 l+3)} V_{l+1}^{0}\right|^{2}+\left[(1-e)^{2}-1\right] \cdot 2 \pi \int d r \sum_{l} \frac{1}{(2 l+1)} . \\
& \mid \frac{l(l-1)}{(2 l-1)}\left(V_{l-1}^{0}+r \frac{\partial V_{l-1}^{0}}{\partial r}\right)-\frac{(l+1)(l+2)}{(2 l+3)} \text {. } \\
& \left.\left(V_{l+1}^{0}+r \frac{\partial V_{l+1}^{0}}{\partial r}\right)\right|^{2}+\left[2-2(1-e)^{2}\right] \cdot 2 \pi \int d r \sum_{l} \frac{1}{(2 l+1)} . \\
& \left|\frac{l^{2}(l-1)}{(2 l-1)} V_{l-1}^{0}+\frac{(l+1)^{2}(l+2)}{(2 l+3)} V_{l+1}^{0}\right| \cdot \mid \frac{l(l-1)}{(2 l-1)} . \\
& \left(V_{l-1}^{0}+r \frac{\partial V_{l-1}^{0}}{\partial r}\right)-\frac{(l+1)(l+2)}{(2 l+3)}\left(V_{l+1}^{0}+r \frac{\partial V_{l+1}^{0}}{\partial r}\right) \\
& E_{\text {tor }}^{\text {axisym }}=2 \pi \int d r \sum_{l} \frac{l(l+1)}{(2 l+1)} r^{4}\left|W_{l}^{0}\right|^{2}
\end{aligned}
$$




\section{Bibliography}

[1] K. D. Aldridge and A. Toomre. "Axisymmetric inertial oscillations of a fluid in a rotating spherical container". J. Fluid Mech., 37: 307-323 (1969).

[2] K. D. Aldridge. "Inertial waves and the Earth's outer core". Geophys. J. R. Astr. Soc., 42: 337-345 (1975).

[3] K. D. Aldridge and L. I. Lumb. "Inertial waves identified in the Earth's fluid outer core". Nature, 325: 421-423 (1975).

[4] K. D. Aldridge, B. Seyed-Mahmoud, G. Henderson and W. van Wijngaarden. "Elliptical instability of the Earth's fluid core". Phys. Earth Planet. Inter., 103: 365-374 (1997).

[5] G. Arfken. Mathematical methods for physicists. Academic Press, Inc. (1985).

[6] B. J. Bayly. "Three-dimensional instability of elliptical flow". Phys. Rev. Lett., 57: 2160-2163 (1986).

[7] H. Bondi and R. A. Lyttleton. "On the dynamical theory of the rotation of the Earth: the effect of precession on the motion of the liquid core". Proc. Camb. Phil. Soc., 49: 498-515 (1953).

[8] V. Borue and S. A. Orszag. "Forced three-dimensional homogeneous turbulence with hyperviscosity". Europhys. Lett., 29: 687-692 (1995).

[9] E. C. Bullard." The magnetic field within the Earth". Proc. Roy. Soc. A, 197: 433453 (1949).

[10] F. H. Busse. "Shear flow instabilities in rotating systems". J. Fluid Mech., 33: 577589 (1968).

[11] F. H. Busse. "Steady fluid flow in a precessing spheroidal shell". J. Fluid Mech., 33: 739-751 (1968).

[12] F. H. Busse. "Magnetohydrodynamics of the Earth's dynamo". Ann. Rev. Fluid Mech., 10: 435-462 (1978). 
[13] F. H. Busse. "Dynamo theory of planetary magnetism and laboratory experiments". In Evolution of dynamical structures in complex systems, Springer Proceedings in Physics, Vol. 69. R. Friedrich and A. Wunderlin editors (1992).

[14] C. Canuto, M. Y. Hussaini, A. Quarteroni and T. A. Zang. Spectral methods in fluid dynamics. Springer, New York (1988).

[15] B. Desjardins, E. Dormy and E. Grenier. "Instability of Ekman-Hartmann boundary layers, with application to the fluid flow near the core-mantle boundary". Phys. Earth Planet. Inter., 123: 15-27 (2001).

[16] M. L. Dudley and R. W. James. "Time-dependent kinematic dynamos with stationary flows". Proc. R. Soc. Lond. A, 425: 407-429 (1989).

[17] G. Dumas and A. Leonard. "A divergence-free spectral expansions method for threedimensional flows in spherical-gap geometries". J. Comp. Phys., 111: 205-219 (1994).

[18] C. Eloy, P. Le Gal and S. Le Dizes. "Experimental study of the multipolar vortex instability". Phys. Rev. Lett., 85: 3400-3403 (2000).

[19] P. Fotheringham and R. Hollerbach. "Inertial oscillations in a spherical shell". Geophys. Astrophys. Fluid Dyn., 89: 23-48 (1998).

[20] P. A. Gilman. "Instabilities of the Ekman-Hartmann boundary layer". Physics of Fluids, 14: 7-12 (1971).

[21] G. A. Glatzmaier. "Numerical simulations of stellar convective dynamos.I.The model and method". J. Comp. Phys., 55: 461-484 (1984).

[22] G. A. Glatzmaier and P. H. Roberts. "A three-dimensional convective dynamo solution with rotating and finitely conducting inner core and mantle". Phys. Earth Planet. Inter., 91: 63-75 (1995).

[23] E. B. Gledzer, F. V. Dolzhanskiy, A. M. Obukhov and V. M. Ponomarev. "An experimental and theoretical study of the stability of motion of a liquid in an elliptical cylinder". Isv. Atmos. and Oceanic Phys., 11: 617-622 (1975).

[24] E. B. Gledzer and V. M. Ponomarev. "Instability of bounded flows with elliptical streamlines". J. Fluid Mech., 240: 1-30 (1992).

[25] H. P. Greenspan. The theory of rotating fluids. Cambridge University Press, Cambridge (1968).

[26] R. Hide and C. W. Titman. "Detached shear layers in a rotating fluid". J. Fluid Mech., 29: 39-60 (1967).

[27] R. Hollerbach and R. R. Kerswell. "Oscillatory internal shear layers in rotating and precessing flows". J. Fluid Mech., 298: 327-339 (1995). 
[28] J. A. Jacobs, editor. Geomagnetism. Vol.2. Academic Press (1987).

[29] R. R. Kerswell. "Elliptical instabilities of stratified, hydromagnetic waves". Geophys. Astrophys. Fluid Dyn., 71: 105-143 (1993).

[30] R. R. Kerswell. "The instability of precessing flow". Geophys. Astrophys. Fluid Dyn., 72: 107-144 (1993).

[31] R. R. Kerswell. "On the internal shear layers spawned by the critical regions in oscillatory Ekman boundary layers". J. Fluid Mech., 298: 311-325 (1995).

[32] R. R. Kerswell and C. F. Barenghi. "On the viscous decay rates of inertial waves in a rotating circular cylinder". J. Fluid Mech., 285: 203-214 (1995).

[33] R. R. Kerswell. "Upper bounds on the energy dissipation in turbulent precession". J. Fluid Mech., 321: 335-370 (1996).

[34] R. R. Kerswell. "Secondary instabilities in rapidly rotating fluids: inertial wave breakdown". J. Fluid Mech., 382: 283-306 (1999).

[35] E. Knittle and R. Jeanloz. "High-pressure metallization of $\mathrm{FeO}$ and implications for the Earth's core". Geophys. Res. Lett., 13: 1541-1544 (1986).

[36] M. D. Kudlick. "On transient motions in a contained, rotating fluid". Ph.D. Thesis, M.I.T. (1966).

[37] H. Lamb. Hydrodynamics. Cambridge University Press, Cambridge (1932).

[38] M. J. Landmann and P. G. Saffman. "The three-dimensional instability of strained vortices in a viscous fluid". Phys. Fluids, 30: 2339-2342 (1987).

[39] S. Leibovich and S. K. Lele. "The influence of the horizontal component of Earth's angular velocity on the instability of the Ekman layer". J. Fluid Mech., 150: 41-87 (1985).

[40] D. K. Lilly. "On the instability of Ekman boundary flow". J. Atmos. Sci., 23: 481-494 (1966).

[41] S. Lorenzani and A. Tilgner. "Precession driven flow in ellipsoidal cavities". In E. Krause and W. Jäger, editors. High performance computing in science and engineering 2000, Springer (2001).

[42] S. Lorenzani and A. Tilgner. "Fluid instabilities in precessing spheroidal cavities". J. Fluid Mech., 447: 111-128 (2001).

[43] W. V. R. Malkus. "Precession of the Earth as the cause of geomagnetism". Science, 169: 259-264 (1968). 
[44] W. V. R. Malkus. "Do precessional torques cause geomagnetism?". In Mathematical problems in the Geophysical Sciences, Lectures in Applied Mathematics, Vol.14: 207228 (1971).

[45] W. V. R. Malkus. "An experimental study of the global instabilities due to the tidal (elliptical) distortion of a rotating elastic cylinder". Geophys. Astrophys. Fluid Dyn., 48: 123-134 (1989).

[46] R. Manasseh. "Breakdown regimes of inertia waves in a precessing cylinder". J. Fluid Mech., 243: 261-296 (1992).

[47] D. M. Mason and R. R. Kerswell. "Nonlinear evolution of the elliptical instability: an example of inertial wave breakdown". J. Fluid Mech., 396: 73-108 (1999).

[48] P. M. Matthews, B. A. Buffet, T. A. Herring and I. I. Shapiro. "Forced nutations of the Earth: influence of inner core dynamics.2.Numerical results and comparisons". J. Geophys. Res., 96: 8243-8257 (1991).

[49] A. D. McEwan. "Inertial oscillations in a rotating fluid cylinder". J. Fluid Mech., 40: 603-640 (1970).

[50] A. D. McEwan. "Forced resonant second-order interaction between damped internal waves". J. Fluid Mech., 55: 589-608 (1972).

[51] H. K. Moffatt. Magnetic field generation in electrically conducting fluids. Cambridge University Press, Cambridge (1978).

[52] J. Noir, D. Jault and P. Cardin. "Numerical study of the motions within a slowly precessing sphere at low Ekman number". J. Fluid Mech., 437: 283-299 (2001).

[53] J. Pedlosky. Geophysical fluid dynamics. Springer-Verlag, New York (1979).

[54] R. T. Pierrehumbert. "Universal short-wave instability of two-dimensional eddies in an inviscid fluid". Phys. Rev. Lett., 57: 2157-2159 (1986).

[55] H. Poincaré. "Sur la précession des corps déformables". Bull. astronom., 27: 321-356 (1910).

[56] J. P. Poirier. "Transport properties of liquid metals and viscosity of the Earth's core". Geophys. J., 92: 99-105 (1988).

[57] W. H. Press, S. A. Teukolsky, W. T. Vetterling and B. P. Flannery. Numerical Recipes. Cambridge University Press, Cambridge (1986).

[58] M. Rieutord. "Inertial modes in the liquid core of the Earth". Phys. Earth Planet. Inter., 91: 41-46 (1995). 
[59] M. Rieutord and L. Valdettaro. "Inertial waves in a rotating spherical shell". J. Fluid Mech., 341: 77-99 (1997).

[60] P. H. Roberts and K. Stewartson. "On the stability of a MacLaurin spheroid of small viscosity". Astrophys. J., 137: 777-790 (1963).

[61] P. H. Roberts and K. Stewartson. "On the motion of a liquid in a spheroidal cavity of a precessing rigid body.II.". Proc. Camb. Phil. Soc., 61: 279-288 (1965).

[62] P. H. Roberts and A. M. Soward, editors. Rotating fluids in geophysics. Academic Press, London (1978).

[63] K. Stewartson. "On almost rigid rotations". J. Fluid Mech., 3: 17-26 (1957).

[64] K. Stewartson. "On almost rigid rotations.II.". J. Fluid Mech., 26: 145-161 (1966).

[65] K. Stewartson and P. H. Roberts. "On the motion of a liquid in a spheroidal cavity of a precessing rigid body". J. Fluid Mech., 17: 1-20 (1963).

[66] P. R. Tatro and E. L. Mollö-Christensen. "Experiments on Ekman layer instability". J. Fluid Mech., 28: 531-543 (1967).

[67] A. Tilgner. "A kinematic dynamo with a small scale velocity field". Phys. Lett. A, 226: $75-79$ (1997).

[68] A. Tilgner. "On models of precession driven core flow". Studia geoph. et geod., 42: 1-7 (1998).

[69] A. Tilgner. "Driven inertial oscillations in spherical shells". Phys. Rev. E, 59: 17891794 (1999).

[70] A. Tilgner. "Magnetohydrodynamic flow in precessing spherical shells". J. Fluid Mech., 379: 303-318 (1999).

[71] A. Tilgner. "Non-axisymmetric shear layers in precessing fluid ellipsoidal shells". Geophys. J. Int., 136: 629-636 (1999).

[72] A. Tilgner. "Spectral methods for the simulation of incompressible flows in spherical shells". Int. J. Numer. Meth. Fluids, 30: 713-724 (1999).

[73] A. Tilgner and F. H. Busse. "Fluid flows in precessing spherical shells". J. Fluid Mech., 426: 387-396 (2001).

[74] D. J. Tritton. Physical fluid dynamics. Clarendon Press, Oxford (1988).

[75] J. P. Vanyo. "A geodynamo powered by luni-solar precession". Geophys. Astrophys. Fluid Dyn., 59: 209-234 (1991). 
[76] J. P. Vanyo, P. Wilde, P. Cardin and P. Olson. "Experiments on precessing flows in the Earth's liquid core". Geophys. J. Int., 121: 136-142 (1995).

[77] J. P. Vanyo and J. Dunn. "Core precession: flow structures and energy". Geophys. J. Int., 142: 409-425 (2000).

[78] F. Waleffe. "On the three-dimensional instability of strained vortices". Phys. Fluids A, 2: 76-80 (1990).

[79] I. C. Walton. "Viscous shear layers in an oscillating rotating fluid". Proc. Roy. Soc. Lond. A, 344: 101-110 (1975).

[80] R. E. Young. "Finite-amplitude thermal convection in a spherical shell". J. Fluid Mech., 63: 695-721 (1974). 


\section{Acknowledgments}

I would like to thank Professor A. Tilgner for his careful, stimulating and very kind supervision of my Ph.D. research work. It was a great pleasure and a privilege for me to work in close collaboration with him.

I would like to thank Professor F. Busse who gave to me the possibility to improve my knowledge in the stimulating atmosphere of the mathematical and physical department of the University of Bayreuth.

I am very grateful to Dr. M. Rein for his kind collaboration as referee of my thesis as well as to Professor R. Kreß, Professor G. Hegerfeldt, Professor G. Lube and Dr. K.-H. Rehren who gave their contribution as members of my examining board. 



\section{Lebenslauf:}

\section{Persönliche Daten}

Name:

Silvia Lorenzani

Geburtsdatum:

23. Juni 1971

Geburtsort:

Terni, Italien

Staatsangehörigkeit: Italienerin

\section{Ausbildung}

09.1977-06.1990

Einschulung in Terni (Italien)

07.1990

Abitur an Gymnasium "G. Tacito" in Terni

ab 11.1990

Studium der Physik an der Universität Bologna (Italien)

10.1997

Diplomprüfung Physik ("Laurea"). Thema der Arbeit:" Modello per l'emissione e la diffusione in atmosfera di un gas pesante" ("A model for heavy gas dispersion in the atmosphere")

$11.1997-08.1998$

Anstellung als wissenschaftlicher Mitarbeiter am Institut FISBAT (CNR) in Bologna

09.1998-03.2001

Anstellung als wissenschaftlicher Mitarbeiter am Institut für Physik der Universität Bayreuth,

ab 04.2001

mit Dienstort in Göttingen. Thema der Doktorarbeit:" Fluid instabilities in precessing ellipsoidal shells" 